\title{
N-Alkynylthio Phthalimide: A Shelf-stable Alkynylthio Transfer Reagent for the Synthesis of Alkynyl Thioethers
}

Wen-Chao Gao, ${ }^{a c *}$ Yu-Zhu Shang, ${ }^{a}$ Hong-Hong Chang, ${ }^{a}$ Xing Li, ${ }^{a}$ Wen-Long Wei, ${ }^{a}$ Xin-Zhang Yu,${ }^{b}$ and Rong Zhou ${ }^{a *}$

\footnotetext{
${ }^{a}$ Taiyuan University of Technology, Taiyuan 030024, P. R. China

${ }^{b}$ Taiyuan Institute of Technology, Taiyuan, 030008, P. R. China
}

${ }^{c}$ Shanghai Key Laboratory of Green Chemistry and Chemical Processes, East China Normal University, Shanghai 200062, P. R. China

*E-mail: gaowenchao@tyut.edu.cn; zhourong@,tyut.edu.cn

\section{Supporting Information}

\section{Table of Contents}

General Information. S2

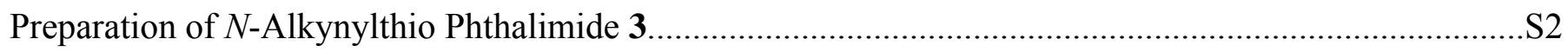

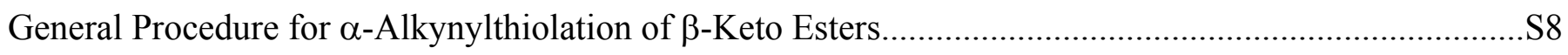

General procedure for $\mathrm{Cu}-\mathrm{Catalyzed} \mathrm{Alkynylthiolation} \mathrm{of} \mathrm{Boronic} \mathrm{acids..............................................S14}$

General Procedure for Alkynylthiolation of Grignard Reagents...........................................................S17

Scheme S1 Reaction of Alcarazo Reagents with $\beta$-Keto Esters and Boronic acids.................................S20

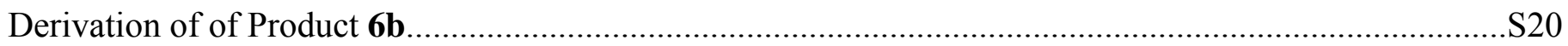

Table S1. Condition Optimization for $\alpha$-Alkynylthiolation of $\beta$-Keto Esters...........................................S22

Table S2. Condition Optimization for $\mathrm{Cu}$-Catalyzed Alkynylthiolation of Boronic acids..........................S23

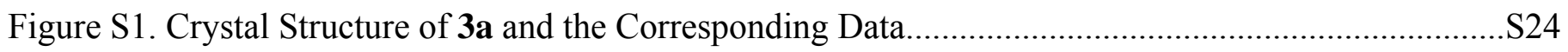

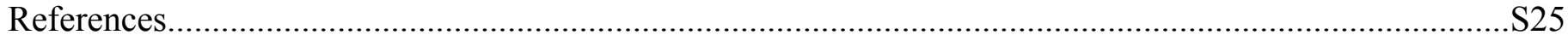

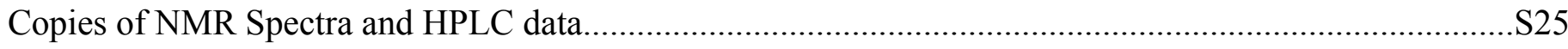




\section{General Information}

${ }^{1} \mathrm{H}$ NMR spectra were recorded at $400 \mathrm{MHz}$ or $500 \mathrm{MHz}$ and ${ }^{13} \mathrm{C}$ NMR spectra were measured at $100 \mathrm{MHz}$ or $125 \mathrm{MHz}$ using Bruker AVANCE III NMR spectrometers with $\mathrm{CDCl}_{3}$ as the solvent. Chemical shifts $(\delta)$ were measured in ppm and referenced to the deuterated chloroform $\left({ }^{1} \mathrm{H}: \delta=7.26 \mathrm{ppm},{ }^{13} \mathrm{C}: \delta=77.00 \mathrm{ppm}\right)$. The multiplicities of signals were described using the following abbreviations: $\mathrm{s}=$ singlet, $\mathrm{d}=$ doublet, $\mathrm{t}$ =triplet, $\mathrm{q}=$ quartet, $\mathrm{m}=$ multiplet, $\mathrm{dd}=$ doublet of doublets, $\mathrm{tt}=$ triplet of triplets. High-resolution mass spectra (HRMS) were performed on a micrOTOF-Q II instrument with an ESI source. Melting points were measured with a RD-II type melting point apparatus. The known compounds were identified by the comparison of their NMR spectra with reported data in literatures; the new compounds were characterized by NMR, HRMS and melting point for solid samples. Unless otherwise noted, reagents obtained from commercial sources were directly used without further purification; all solvents were obtained from commercial sources and were purified according to standard procedures. Petroleum ether (PE), where used, has the boiling point range $60-90^{\circ} \mathrm{C}$. Column chromatography was performed on silica gel (200-300 mesh). The HPLC measurements were carried out on an Elite Agress 1100 apparatus. The used solvents were hexane and 2-propanol and were bought from Energy Chemical as HPLC grade. The chiral column used for the separation of the enantiomers was Daicel Chiralcel AS-H $(0.46 \mathrm{~cm} \varnothing \times 25 \mathrm{~cm})$.

\section{Preparation of $N$-Alkynylthio Phthalimide 3}

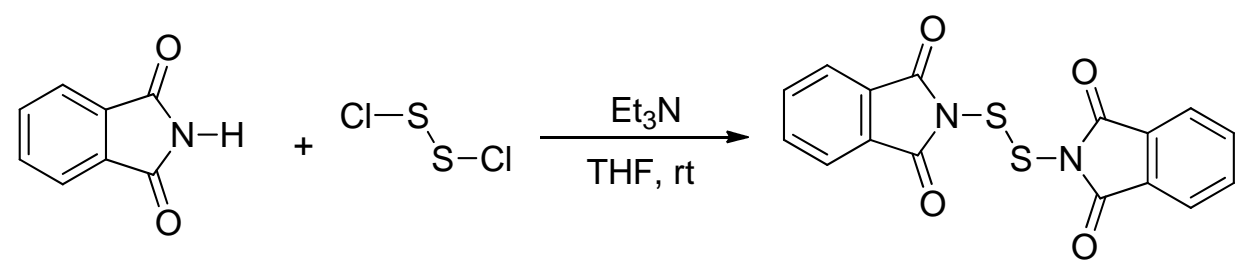

Phthalimide (2.9 g, $19.7 \mathrm{mmol})$ was dissolved in THF $(40 \mathrm{~mL})$ and triethylamine $(4.1 \mathrm{~mL}, 30 \mathrm{mmol})$. The mixture was cooled in a salt ice bath, and then sulfur monochloride $(0.8 \mathrm{~mL}, 10 \mathrm{mmol})$ was added dropwise to the cooled mixture. The solution was stirred for $2 \mathrm{~h}$, and then quenched with $60 \mathrm{~mL}$ of $\mathrm{H}_{2} \mathrm{O}$. The resulting precipitate was filtered and washed with diethyl ether. The resulting precipitate was collected and dissolved in the solvent of $\mathrm{CHCl}_{3}: \mathrm{CH}_{3} \mathrm{OH}[2: 1(\mathrm{v}: \mathrm{v}), 45 \mathrm{~mL}]$, and reflux for $0.5-1 \mathrm{~h}$. The insoluble solid was removed, and the filtrate was concentrated under vacuum to give 2,2'-disulfanediylbis(isoindoline-1,3-dione) as white crystal (3.5g, yield: 98\%). The NMR spectra are consistent with literature reports. ${ }^{1}$ 


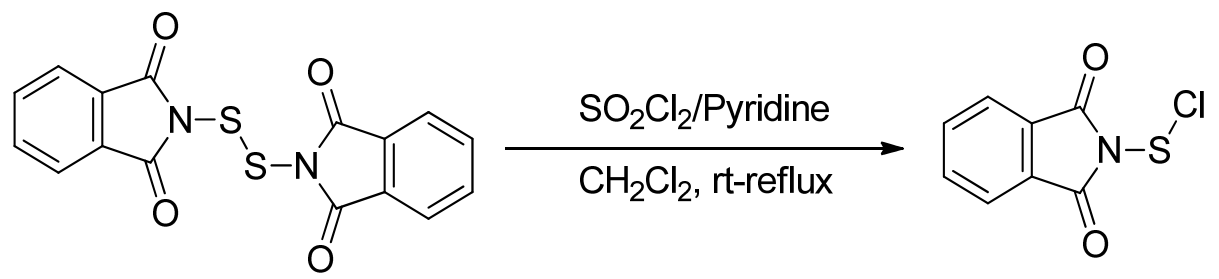

Following a slightly modified procedure described in literatures,${ }^{2}$ to a solution of di(1-phthalimidy1)disulfane (750 mg, $2 \mathrm{mmol})$ and anhydrous pyridine $(0.1 \mathrm{~mL}, 1.2 \mathrm{mmol})$ in $10 \mathrm{~mL}$ of $\mathrm{CH}_{2} \mathrm{Cl}_{2}$ was added sulfuryl chloride $(2.5 \mathrm{~mL}, 31 \mathrm{mmol})$ dropwise at room temperature. The yellow mixture was stirred at room temperature for 24 $\mathrm{h}$, and reflux for $4 \mathrm{~h}$ (Note: Please ensure no water come inside during this period!). The solvent and excess sulfuryl chloride were removed under vacuum, and the resulting solid was dissolved in $15 \mathrm{~mL}_{\text {of }} \mathrm{CCl}_{4}$ at reflux for $0.5 \mathrm{~h}$. The insoluble precipitate was removed, and the filtrate was concentrated to give a yellow solid (380 mg, yield: 90\%).

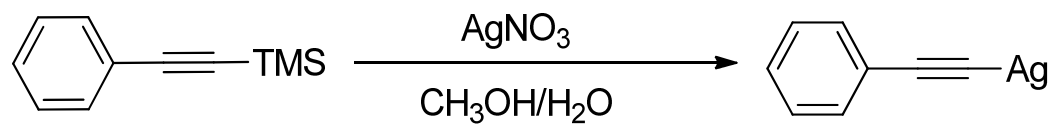

To a solution of 1-trimethylsilyl-2-phenylacetylene $(5 \mathrm{mmol})$ in $20 \mathrm{~mL}$ methanol $\left(\mathrm{H}_{2} \mathrm{O}: \mathrm{MeOH}=1: 3\right)$, was added silver nitrate $(5 \mathrm{mmol})$ at room temperature. The starting materials rapidly disappeared and a white precipitate formed within 5-15 min. This solid was recovered by filtration and washed with cold $\mathrm{MeOH}$ and $\mathrm{CH}_{2} \mathrm{Cl}_{2}$ (stored in dark at $0{ }^{\circ} \mathrm{C}$ ). Subsequent drying led to the phenylacetylene silver $(947 \mathrm{mg}$, yield: $91 \%$ ) as a white powder. ${ }^{3 a}$

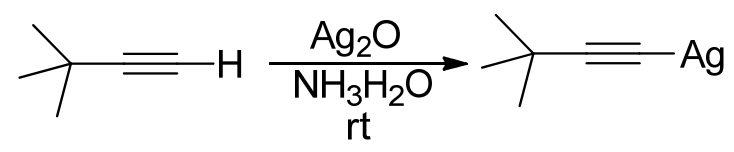

The aliphatic silver acetylides are prepared using an alternative method. ${ }^{3 \mathrm{~b}} \mathrm{Ag}_{2} \mathrm{O}(3.00 \mathrm{~g}, 12.9 \mathrm{mmol})$ was dissolved in ammonia $(60 \mathrm{ml})$ under stirring. $\mathrm{t}-\mathrm{BuC} \equiv \mathrm{CH}(1.50 \mathrm{~g}, 18.3 \mathrm{mmol})$ in ethanol $(5 \mathrm{ml})$ was dropwise added to the silver ammonia solution, white precipitate was generated immediately, which was filtered and washed by water, ethanol and ethyl ether to give $t$-BuC $\equiv \mathrm{CAg}(3.25 \mathrm{~g}$, yield: $95 \%)$.<smiles>CCCCCCC#Cc1ccccc1</smiles><smiles>O=C1c2ccccc2C(=O)N1SC#Cc1ccccc1</smiles>

To a $100 \mathrm{~mL}$ round-bottomed flask charged with $N$-(Chlorosulfenyl)phthalimide (256 $\mathrm{mg}, 1.2 \mathrm{mmol})$ and phenylacetylene silver $(250 \mathrm{mg}, 1.2 \mathrm{mmol})$ was added dichloromethane $(10 \mathrm{~mL})$. The mixture was stirred vigorously at room temperature for $120 \mathrm{~min}$ and then filtered through a pad of Celite and washed with 
dichloromethane $(50 \mathrm{~mL})$. The filtrate was concentrated in vacuo and purified by chromatography (PE:EtOAc $=1: 5)$ to give 3a $(240 \mathrm{mg}, 72 \%)$. pale yellow solid, m.p.:133-135 ${ }^{\circ} \mathrm{C}$.

\section{Gram-Scale Synthesis of 3a.}

To a solution of $N$-(Chlorosulfenyl)phthalimide $(4.26 \mathrm{~g}, 20 \mathrm{mmol})$ in anhydrous dichloromethane $(100 \mathrm{~mL})$ was added phenylacetylene silver $(4.2 \mathrm{~g}, 20 \mathrm{mmol})$ in one portion. The mixture was stirred at room temperature for $4 \mathrm{~h}$ and then filtered through a pad of Celite and washed with dichloromethane $(100 \mathrm{~mL} \times 3)$. The filtrate was evaporated in vacuo and purified by flash chromatography to give $3 a$ ( 3.3 g, yield: $60 \%)$ as pale yellow solid.

\section{2-((Phenylethynyl)thio)isoindoline-1,3-dione (3a)}<smiles>O=C1c2ccccc2C(=O)N1SC#Cc1ccccc1</smiles>

Yield: $240 \mathrm{mg}$ (72\%); time: $1.5 \mathrm{~h}$; yellow solid; $\mathrm{mp} 133-135{ }^{\circ} \mathrm{C}$; TLC, $\mathrm{R}_{\mathrm{f}}=0.34(\mathrm{PE}: \mathrm{EtOAc}=9: 1) ;{ }^{1} \mathrm{H}$ NMR $\left(\mathrm{CDCl}_{3}, 500 \mathrm{MHz}\right): \delta$ 7.98-7.96 (m, 2H), 7.82-7.7.80 (m, 2H), 7.46-7.44 (m, 2H), 7.35-7.33 (m, 1H), 7.31$7.29(\mathrm{~m}, 2 \mathrm{H}) ;{ }^{13} \mathrm{C}\left\{{ }^{1} \mathrm{H}\right\} \mathrm{NMR}\left(\mathrm{CDCl}_{3}, 125 \mathrm{MHz}\right): \delta 166.4,134.9,132.6,132.1,129.7,128.4,124.3,121.5$, 101.5; HRMS (ESI) $\mathrm{m} / z$ calcd. for $\mathrm{C}_{16} \mathrm{H}_{9} \mathrm{NO}_{2} \mathrm{SNa}[\mathrm{M}+\mathrm{Na}]^{+}: 302.0246$, found: 302.0242 .

\section{2-(((3-Fluorophenyl)ethynyl)thio)isoindoline-1,3-dione (3b)}

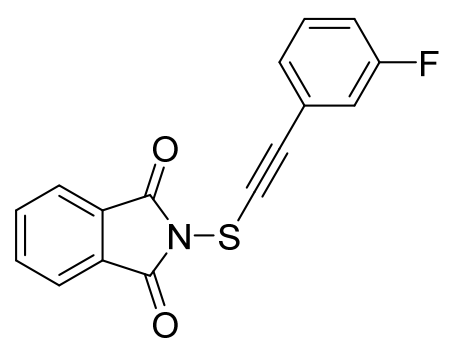

Yield:267 mg (75\%); time: $1.5 \mathrm{~h}$; yellow solid; $\mathrm{mp} 155-157{ }^{\circ} \mathrm{C} ; \mathrm{R}_{\mathrm{f}}=0.33(\mathrm{PE}:$ EtOAc $=9: 1) ;{ }^{1} \mathrm{H}$ NMR (CDCl3,400 MHz): $\delta$ 8.00-7.49 (m, 2H), 7.84-7.82 (m, 2H), 7.30-7.21 (m, 2H), 7.15-7.12 (m, 1H), 7.08-7.03 $(\mathrm{m}, 1 \mathrm{H}) ;{ }^{13} \mathrm{C}\{1 \mathrm{H}\}$ NMR $(\mathrm{CDCl} 3,100 \mathrm{MHz}): \delta 166.3,162.1(J=246.0 \mathrm{~Hz}), 134.9,134.3,132.0,130.0(J=$ $9.0 \mathrm{~Hz}), 128.22,128.19,124.3,123.6,123.2(\mathrm{~J}=9.0 \mathrm{~Hz}) 119.0(\mathrm{~J}=23.0 \mathrm{~Hz}), 117.0(\mathrm{~J}=21.0 \mathrm{~Hz}), 99.9,78.1$; HRMS (ESI) m/z calcd. for $\mathrm{C}_{16} \mathrm{H}_{8} \mathrm{FNO}_{2} \mathrm{SNa}[\mathrm{M}+\mathrm{Na}]^{+}: 320.0152$, found: 320.0153 . 


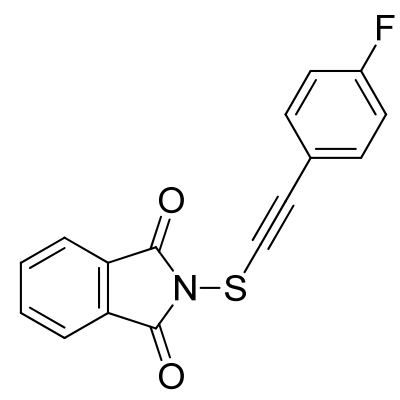

Yield: $278 \mathrm{mg}$ (78\%); time: $1.5 \mathrm{~h}$; yellow solid; $\mathrm{mp} 157-159{ }^{\circ} \mathrm{C}$; TLC, $\mathrm{R}_{\mathrm{f}}=0.33(\mathrm{PE}: \mathrm{EtOAc}=9: 1) ;{ }^{1} \mathrm{H}$ NMR $\left(\mathrm{CDCl}_{3}, 400 \mathrm{MHz}\right): \delta$ 7.98-7.96 (m, 2H), 7.82-7.80 (m, 2H), 7.48-7.44 (m, 2H), 7.02-6.97 (m, 2H); ${ }^{13} \mathrm{C}\left\{{ }^{1} \mathrm{H}\right\}$ NMR ( $\left.\mathrm{CDCl}_{3}, 100 \mathrm{MHz}\right): \delta$ 166.4, $163.4(\mathrm{~J}=251.0 \mathrm{~Hz}), 135.0(\mathrm{~J}=9.0 \mathrm{~Hz}), 134.3,132.0,124.3,123.6,117.6$ $(J=4.0 \mathrm{~Hz}), 115.8(J=23.0 \mathrm{~Hz}), 100.4$; HRMS $(\mathrm{ESI}) \mathrm{m} / \mathrm{z}$ calcd. for $\mathrm{C}_{16} \mathrm{H}_{8} \mathrm{FNO}_{2} \mathrm{SNa}[\mathrm{M}+\mathrm{Na}]^{+}: 320.0152$, found: 320.0153 .

2-((p-Tolylethynyl)thio)isoindoline-1,3-dione (3d)

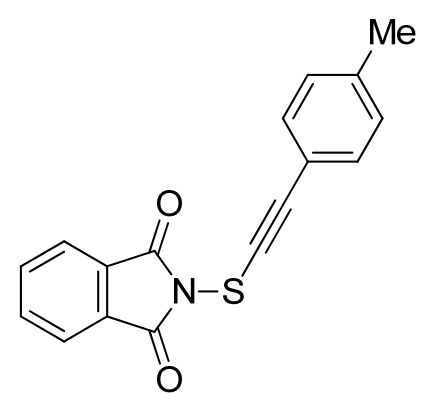

Yield: $250 \mathrm{mg}$ (71\%); time: $1.0 \mathrm{~h}$; yellow solid; $\mathrm{mp} 190-192{ }^{\circ} \mathrm{C}$; $\mathrm{TLC}, \mathrm{R}_{\mathrm{f}}=0.32(\mathrm{PE}: \mathrm{EtOAc}=9: 1) ;{ }^{1} \mathrm{H}$ NMR $\left(\mathrm{CDCl}_{3}, 400 \mathrm{MHz}\right): \delta$ 7.98-7.95 (m,2H), 7.81-7.79 (m, 2H), 7.36 (d, 2H, J = 8.0 Hz), $7.10(\mathrm{~d}, 2 \mathrm{H}, \mathrm{J}=7.6 \mathrm{~Hz})$, $2.33(\mathrm{~s}, 3 \mathrm{H}) ;{ }^{13} \mathrm{C}\left\{{ }^{1} \mathrm{H}\right\} \mathrm{NMR}\left(\mathrm{CDCl}_{3}, 100 \mathrm{MHz}\right): \delta 166.5,140.4,134.8,132.8,132.0,129.1,124.2,118.4,101.9$, 76.2, 21.6; HRMS (ESI) m/z calcd. for $\mathrm{C}_{17} \mathrm{H}_{11} \mathrm{NO}_{2} \mathrm{SNa}[\mathrm{M}+\mathrm{Na}]^{+}: 316.0403$, found: 316.0407 .

2-((Naphthalen-1-ylethynyl)thio)isoindoline-1,3-dione (3e)

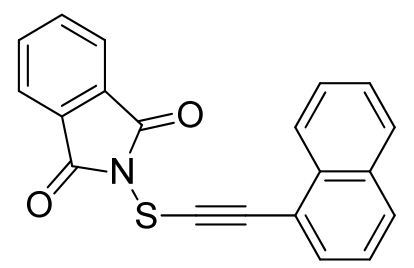

Yield: $217 \mathrm{mg}$ (55\%); time: $1.2 \mathrm{~h}$; brown solid; $\mathrm{mp} 152-154{ }^{\circ} \mathrm{C}$; $\mathrm{TLC}, \mathrm{R}_{\mathrm{f}}=0.32(\mathrm{PE}: \mathrm{EtOAc}=9: 1) ;{ }^{1} \mathrm{H}$ NMR $\left(\mathrm{CDCl}_{3}, 400 \mathrm{MHz}\right): \delta 8.27(\mathrm{~d}, 1 \mathrm{H}, \mathrm{J}=8.4 \mathrm{~Hz}), 7.98-7.95(\mathrm{~m}, 2 \mathrm{H}), 7.84(\mathrm{t}, 2 \mathrm{H}, \mathrm{J}=8.4 \mathrm{~Hz}), 7.80-7.78(\mathrm{~m}, 2 \mathrm{H})$, $7.71(\mathrm{dd}, 1 \mathrm{H}, \mathrm{J}=6.8,0.8 \mathrm{~Hz}) 7.60-7.56(\mathrm{~m}, 1 \mathrm{H}), 7.53-7.48(\mathrm{~m}, 1 \mathrm{H}), 7.39(\mathrm{t}, 1 \mathrm{H}, \mathrm{J}=8.0 \mathrm{~Hz}) ;{ }^{13} \mathrm{C}\left\{{ }^{1} \mathrm{H}\right\} \mathrm{NMR}$ $\left(\mathrm{CDCl}_{3}, 100 \mathrm{MHz}\right): \delta 166.4,133.6,132.9,132.4,132.0,130.5,128.2,127.3,126.6,125.9,125.0,124.2,119.0$, 99.7, 81.5; HRMS (ESI) m/z calcd. for $\mathrm{C}_{20} \mathrm{H}_{11} \mathrm{NO}_{2} \mathrm{SNa}[\mathrm{M}+\mathrm{Na}]^{+}: 282.0559$, found: 282.0568 .

\section{2-((Thiophen-3-ylethynyl)thio)isoindoline-1,3-dione (3f)}




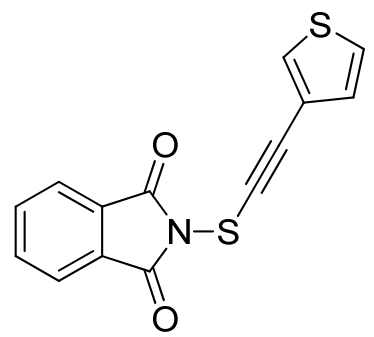

Yield: $157 \mathrm{mg}$ (46\%); time: $1.0 \mathrm{~h}$; yellow solid; $\mathrm{mp} 140-142{ }^{\circ} \mathrm{C}$; $\mathrm{TLC}, \mathrm{R}_{\mathrm{f}}=0.30(\mathrm{PE}: \mathrm{EtOAc}=9: 1) ;{ }^{1} \mathrm{H}$ NMR $\left(\mathrm{CDCl}_{3}, 400 \mathrm{MHz}\right): \delta$ 7.97-7.95 $(\mathrm{m}, 2 \mathrm{H}), 7.81-7.79(\mathrm{~m}, 2 \mathrm{H}), 7.61-7.60(\mathrm{~m}, 1 \mathrm{H}), 7.25-7.23(\mathrm{~m}, 1 \mathrm{H}), 7.12(\mathrm{~d}$, $1 \mathrm{H}, \mathrm{J}=5.2 \mathrm{~Hz}) ;{ }^{13} \mathrm{C}\left\{{ }^{1} \mathrm{H}\right\} \mathrm{NMR}\left(\mathrm{CDCl}_{3}, 100 \mathrm{MHz}\right): \delta 166.4,134.8,132.8,132.0,130.4,125.5,124.2,120.7$, 96.6; HRMS (ESI) m/z calcd. for $\mathrm{C}_{14} \mathrm{H}_{7} \mathrm{NO}_{2} \mathrm{~S}_{2} \mathrm{Na}[\mathrm{M}+\mathrm{Na}]^{+}$: 307.9810, found: 307.9818 .

\section{2-((Cyclopropylethynyl)thio)isoindoline-1,3-dione (3g)}

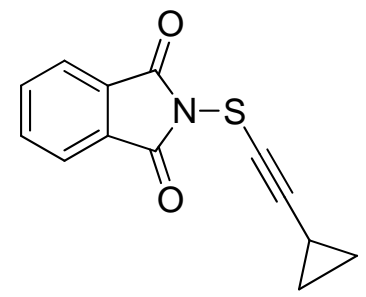

Yield: $192 \mathrm{mg}$ (66\%); time: $1.2 \mathrm{~h}$; yellow solid; mp 152-154 ${ }^{\circ} \mathrm{C} ; \mathrm{TLC}, \mathrm{R}_{\mathrm{f}}=0.18(\mathrm{PE}: \mathrm{EtOAc}=9: 1) ;{ }^{1} \mathrm{H}$ NMR $\left(\mathrm{CDCl}_{3}, 400 \mathrm{MHz}\right): \delta$ 7.95-7.93 (m, 2H), 7.80-7.78 (m, 2H), 1.40-1.34 (m, 1H), 0.88-0.78 (m, 4H); ${ }^{13} \mathrm{C}\left\{{ }^{1} \mathrm{H}\right\}$ NMR $\left(\mathrm{CDCl}_{3}, 100 \mathrm{MHz}\right): \delta 166.5,134.8,132.0,124.2,109.8,63.8,9.9,0.8 ; \mathrm{HRMS}$ (ESI) m/z calcd. for $\mathrm{C}_{13} \mathrm{H}_{9} \mathrm{NO}_{2} \mathrm{SNa}[\mathrm{M}+\mathrm{Na}]^{+}: 266.0246$, found: 266.0249 .

\section{2-((3,3-Dimethylbut-1-yn-1-yl)thio)isoindoline-1,3-dione (3h)}

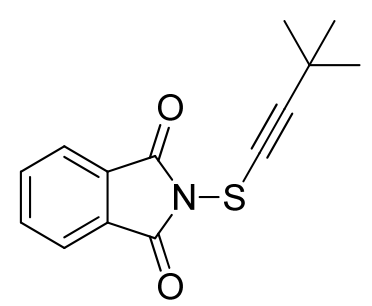

Yield: $195 \mathrm{mg}$ (63\%); time: $1.5 \mathrm{~h}$; yellow solid; $\mathrm{mp} 124-126{ }^{\circ} \mathrm{C}$; TLC, $\mathrm{R}_{\mathrm{f}}=0.34(\mathrm{PE}: \mathrm{EtOAc}=9: 1) ;{ }^{1} \mathrm{H}$ NMR $\left(\mathrm{CDCl}_{3}, 400 \mathrm{MHz}\right): \delta$ 7.97-7.95 (m, 2H), 7.81-7.79 (m, 2H), $1.19(\mathrm{~s}, 9 \mathrm{H}) ;{ }^{13} \mathrm{C}\left\{{ }^{1} \mathrm{H}\right\} \mathrm{NMR}(\mathrm{CDCl} 3,100 \mathrm{MHz})$ : $\delta 166.5,134.8,132.1,124.2,111.8,66.1,30.3,28.9$; HRMS (ESI) $\mathrm{m} / \mathrm{z}$ calcd. for $\mathrm{C}_{14} \mathrm{H}_{14} \mathrm{NO}_{2} \mathrm{~S}[\mathrm{M}+\mathrm{H}]^{+}$: 260.0740, found: 260.0741 .

\section{2-(Hex-1-yn-1-ylthio)isoindoline-1,3-dione (3i)}<smiles>CCCCC#CSN1C(=O)c2ccccc2C1=O</smiles> 
Yield: $177 \mathrm{mg}(57 \%)$; time: $1.3 \mathrm{~h}$; yellow solid; mp 74-76 ${ }^{\circ} \mathrm{C}$; TLC, $\mathrm{R}_{\mathrm{f}}=0.40(\mathrm{PE}: \mathrm{EtOAc}=9: 1) ;{ }^{1} \mathrm{H}$ NMR $\left(\mathrm{CDCl}_{3}, 400 \mathrm{MHz}\right): \delta$ 7.97-7.94 (m, 2H), 7.81-7.79 (m, 2H), $2.32(\mathrm{t}, 2 \mathrm{H}, \mathrm{J}=7.2 \mathrm{~Hz}), 1.50-1.43$ (m, 2H), 1.39$1.30(\mathrm{~m}, 2 \mathrm{H}), 0.85(\mathrm{t}, 3 \mathrm{H}, \mathrm{J}=7.6 \mathrm{~Hz}) ;{ }^{13} \mathrm{C}\left\{{ }^{1} \mathrm{H}\right\} \mathrm{NMR}\left(\mathrm{CDCl}_{3}, 100 \mathrm{MHz}\right): \delta 166.6,134.8,132.0,124.2,105.0$, 67.3, 30.0, 21.9, 20.0, 13.5; HRMS (ESI) m/z calcd. for $\mathrm{C}_{14} \mathrm{H}_{13} \mathrm{NO}_{2} \mathrm{SNa}[\mathrm{M}+\mathrm{Na}]^{+}: 282.0559$, found: 282.0568 . 2-(((Triisopropylsilyl)ethynyl)thio)isoindoline-1,3-dione (3j)

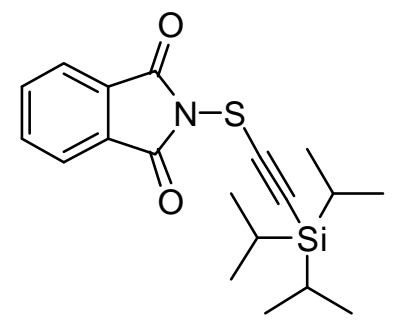

Yield:207.5 mg (80\%); time: $1.0 \mathrm{~h}$; white solid; $\mathrm{mp} 86-88^{\circ} \mathrm{C}$; TLC, $\mathrm{R}_{\mathrm{f}}=0.38(\mathrm{PE}: \mathrm{EtOAc}=9: 1) ;{ }^{1} \mathrm{H}$ NMR $\left(\mathrm{CDCl}_{3}, 400 \mathrm{MHz}\right): \delta$ 7.95-7.91 (m, 2H), 7.82-7.77 (m, 2H), $0.97(\mathrm{~m}, 21 \mathrm{H}) ;{ }^{13} \mathrm{C}\left\{{ }^{1} \mathrm{H}\right\} \mathrm{NMR}\left(\mathrm{CDCl}_{3}, 100 \mathrm{MHz}\right)$ : $\delta$ 166.0, 134.8, 131.9, 124.1, 108.8, 92.4, 18.4, 11.0; HRMS (ESI) m/z calcd. for $\mathrm{C}_{19} \mathrm{H}_{26} \mathrm{NO}_{2} \mathrm{SSi}[\mathrm{M}+\mathrm{H}]^{+}$: 360.1448, found: 360.1432 .

\section{2-((4-Azidobut-1-yn-1-yl)thio)isoindoline-1,3-dione (3k)}

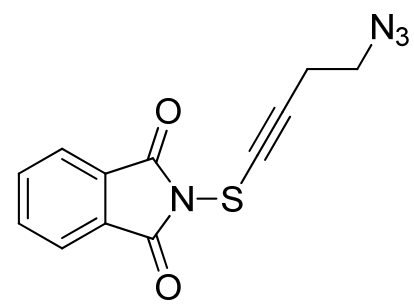

Yield: $212 \mathrm{mg}$ (65\%); time: $1.2 \mathrm{~h}$; brown solid; mp 70-72 ${ }^{\circ} \mathrm{C} ; \mathrm{TLC}, \mathrm{Rf}=0.23$ (PE:EtOAc = 9:1); ${ }^{1} \mathrm{H}$ NMR $\left(\mathrm{CDCl}_{3}, 400 \mathrm{MHz}\right): \delta$ 7.96-7.94 (m, 2H), 7.82-7.79 (m, 2H), $3.37(\mathrm{t}, 2 \mathrm{H}, \mathrm{J}=6.8 \mathrm{~Hz}), 2.62(\mathrm{td}, 2 \mathrm{H}, \mathrm{J}=6.8,0.8$ $\mathrm{Hz}) ;{ }^{13} \mathrm{C}\left\{{ }^{1} \mathrm{H}\right\} \mathrm{NMR}\left(\mathrm{CDCl}_{3}, 100 \mathrm{MHz}\right): \delta 166.4,134.9,132.0,124.3,100.1,70.1,49.2,21.4$; HRMS (ESI) $\mathrm{m} / \mathrm{z}$ calcd. for $\mathrm{C}_{12} \mathrm{H}_{8} \mathrm{~N}_{4} \mathrm{O}_{2} \mathrm{SNa}[\mathrm{M}+\mathrm{Na}]^{+}:$295.0260, found:295.0270.

\section{2-((4-Hydroxybut-1-yn-1-yl)thio)isoindoline-1,3-dione (3l)}

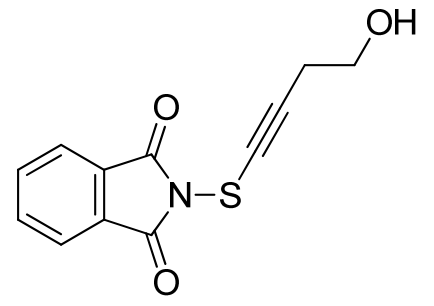

Yield:165.2 mg (42\%); time: $1.0 \mathrm{~h}$; white solid; $\mathrm{mp} 104-106{ }^{\circ} \mathrm{C}$; TLC, $\mathrm{R}_{\mathrm{f}}=0.48(\mathrm{PE}: \mathrm{EtOAc}=7: 3) ;{ }^{1} \mathrm{H} \mathrm{NMR}$ $\left(\mathrm{CDCl}_{3}, 400 \mathrm{MHz}\right): \delta$ 7.96-7.94 (m, 2H), 7.81-7.79 (m, 2H), 3.70 (t, 2H, J = 6.4 Hz), 2.62 (t, 2H, J = 6.4 Hz), $2.06(\mathrm{~s}, 1 \mathrm{H}) ;{ }^{13} \mathrm{C}\left\{{ }^{1} \mathrm{H}\right\} \mathrm{NMR}\left(\mathrm{CDCl}_{3}, 100 \mathrm{MHz}\right): \delta 166.4,134.9,131.9,124.2,101.4,69.6,60.4,24.6$; HRMS 
(ESI) $\mathrm{m} / \mathrm{z}$ calcd. for $\mathrm{C}_{12} \mathrm{H}_{10} \mathrm{NO}_{3} \mathrm{~S}[\mathrm{M}+\mathrm{H}]^{+}:$248.0376, found: 248.0382 .

\section{General Procedure for $\alpha$-Alkynylthiolation of $\beta$-Keto Esters}

To a $10 \mathrm{~mL}$ flask charged with methyl 1-indanone-2-carboxylate $(0.1 \mathrm{mmol}), \mathrm{DABCO}(0.02 \mathrm{mmol})$, and $N$ phenylethynylthio phthalimide $3 \mathbf{a}(0.15 \mathrm{mmol})$ was added dichloromethane $(1 \mathrm{~mL})$. The mixture was stirred at reflux for $2 \mathrm{~h}$. The solvent was removed under vacuum, and the residue was purified by column chromatography $(\mathrm{PE} / \mathrm{EtOAc}=10: 1)$ to give $4 \mathrm{a}$ in $95 \%$ yield.

\section{General Procedure for Enantioselective $\alpha$-Alkynlthiolation of tert-Butyl 1-Indanone-2-carboxylate}

To a $10 \mathrm{~mL}$ flask charged with tert-butyl 1-indanone-2-carboxylate $(0.1 \mathrm{mmol})$, quinine $(0.02 \mathrm{mmol})$, and $N$ phenylethynylthio phthalimide $3 a(0.15 \mathrm{mmol})$ was added dichloromethane $(1 \mathrm{~mL})$. The reaction mixture was stirred at $0{ }^{\circ} \mathrm{C}$ for $3 \mathrm{~h}$. The solvent was removed under vacuum, and the residue was purified by column chromatography $(\mathrm{PE} / \mathrm{EtOAc}=10: 1)$ to give 4c. Yield: 79\%; 70\% ee, determined by HPLC analysis: Daicel Chiralcel AS-H column, hexane $/ i$-PrOH: 399/1, $1.2 \mathrm{~mL} / \mathrm{min}, 20^{\circ} \mathrm{C}, 254 \mathrm{~nm}, \mathrm{~T}$ (major) = $28.7 \mathrm{~min}$, TR (minor) $=39.1 \mathrm{~min} ;[\alpha]_{\mathrm{D}}^{20}=-33\left(\mathrm{c}=0.10, \mathrm{CHCl}_{3}\right)$.

\section{Methyl 1-oxo-2-((phenylethynyl)thio)-2,3-dihydro-1H-indene-2-carboxylate (4a)}

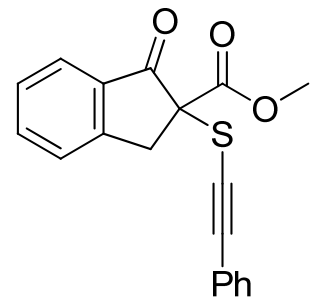

Yield: $30.6 \mathrm{mg}$ (95\%); time: $2.0 \mathrm{~h}$; brown liquid; TLC, $\mathrm{R}_{\mathrm{f}}=0.39(\mathrm{PE}: \mathrm{EtOAc}=9: 1) ;{ }^{1} \mathrm{H} \mathrm{NMR}\left(\mathrm{CDCl}_{3}, 400\right.$ MHz): $\delta 7.86(\mathrm{~d}, 1 \mathrm{H}, \mathrm{J}=7.6 \mathrm{~Hz}), 7.67(\mathrm{t}, 1 \mathrm{H}, \mathrm{J}=7.2 \mathrm{~Hz}), 7.51-7.43(\mathrm{~m}, 2 \mathrm{H}), 7.22-7.15(\mathrm{~m}, 3 \mathrm{H}), 6.96(\mathrm{~d}, 2 \mathrm{H}$, $\mathrm{J}=6.8 \mathrm{~Hz}), 4.07(\mathrm{~d}, 1 \mathrm{H}, \mathrm{J}=17.6 \mathrm{~Hz}) 3.81(\mathrm{~s}, 3 \mathrm{H}), 3.75(\mathrm{~d}, 1 \mathrm{H}, \mathrm{J}=18.0 \mathrm{~Hz}) ;{ }^{13} \mathrm{C}\left\{{ }^{1} \mathrm{H}\right\} \mathrm{NMR}\left(\mathrm{CDCl}_{3}, 100\right.$ MHz): $\delta 196.9,167.9,151.9,136.0,134.2,131.0,128.3,128.2,128.1,126.0,125.3,122.3,97.9,63.7,53.9$, 39.9; HRMS (ESI) m/z calcd. for $\mathrm{C}_{19} \mathrm{H}_{15} \mathrm{O}_{3} \mathrm{~S}[\mathrm{M}+\mathrm{H}]^{+}: 323.0736$, found: 323.0743 .

Ethyl 1-oxo-2-((phenylethynyl)thio)-2,3-dihydro-1H-indene-2-carboxylate (4b)<smiles>CCOC(=O)C1(SC#CC#Cc2ccccc2)Cc2ccccc2C1=O</smiles>

Yield:28.9 mg (86\%); time: 3.0 h; brown liquid; TLC, $\mathrm{R}_{\mathrm{f}}=0.39(\mathrm{PE}: \mathrm{EtOAc}=9: 1) ;{ }^{1} \mathrm{H} \mathrm{NMR}\left(\mathrm{CDCl}_{3}, 400\right.$ 
MHz): $\delta 7.86(\mathrm{~d}, 1 \mathrm{H}, \mathrm{J}=7.6 \mathrm{~Hz}), 7.66(\mathrm{td}, 1 \mathrm{H}, \mathrm{J}=7.6,1.6 \mathrm{~Hz}), 7.51-7.43(\mathrm{~m}, 2 \mathrm{H}), 7.24-7.14(\mathrm{~m}, 3 \mathrm{H}), 6.97-$ $6.95(\mathrm{~m}, 2 \mathrm{H}), 4.31-4.26(\mathrm{~m}, 2 \mathrm{H}), 4.06(\mathrm{~d}, 1 \mathrm{H}, \mathrm{J}=18.0 \mathrm{~Hz}), 3.75(\mathrm{~d}, 1 \mathrm{H}, \mathrm{J}=18.0 \mathrm{~Hz}), 1.27$ (t, 3H, J = 7.2 Hz);

${ }^{13} \mathrm{C}\left\{{ }^{1} \mathrm{H}\right\} \mathrm{NMR}\left(\mathrm{CDCl}_{3}, 100 \mathrm{MHz}\right): \delta 197.1,167.4,151.9,136.0,134.3,131.0,128.1,126.0,125.4,122.4$, 97.7, 75.0 , 63.8, 63.2, 40.0, 14.0; HRMS (ESI) m/z calcd. for $\mathrm{C}_{20} \mathrm{H}_{17} \mathrm{O}_{3}[\mathrm{M}+\mathrm{H}]^{+}:$337.0893, found: 337.0898 . tert-Butyl 1-oxo-2-((phenylethynyl)thio)-2,3-dihydro-1H-indene-2-carboxylate (4c)

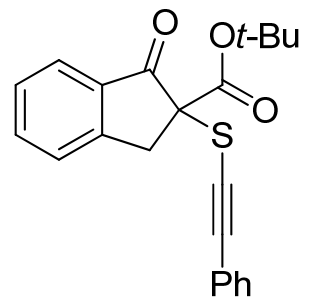

Yield:26.3 mg (87\%); time: 3.0 h; brown liquid; TLC, $\mathrm{R}_{\mathrm{f}}=0.35(\mathrm{PE}: \mathrm{EtOAc}=9: 1)$; ${ }^{1} \mathrm{H}$ NMR $\left(\mathrm{CDCl}_{3}, 400\right.$ MHz): $\delta 7.85(\mathrm{~d}, 1 \mathrm{H}, \mathrm{J}=7.6 \mathrm{~Hz}), 7.66(\mathrm{td}, 1 \mathrm{H}, \mathrm{J}=7.6,1.2 \mathrm{~Hz}), 7.49(\mathrm{~d}, 1 \mathrm{H}, \mathrm{J}=7.6 \mathrm{~Hz}), 7.42(\mathrm{t}, 1 \mathrm{H}, \mathrm{J}=7.2$ Hz), 7.21-7.15 (m, 3H), 6.94-6.92 (m, 2H), 4.00 (d, 1H, J =17.6 Hz), 3.72 (d, 1H, J = 17.6Hz), 1.47 (s, 9H); ${ }^{13} \mathrm{C}\left\{{ }^{1} \mathrm{H}\right\} \mathrm{NMR}\left(\mathrm{CDCl}_{3}, 100 \mathrm{MHz}\right): \delta 197.6,166.4,152.0,134.6,130.9,128.1,128.05,128.03,125.9,125.3$, 122.6, 97.3, 84.4,75.5, 64.6, 40.0, 27.8; HRMS (ESI) m/z calcd. for $\mathrm{C}_{22} \mathrm{H}_{21} \mathrm{O}_{3} \mathrm{~S}[\mathrm{M}+\mathrm{H}]^{+}:$382.1471, found: 382.1470 .

Adamantan-1-yl 1-oxo-2-((phenylethynyl)thio)-2,3-dihydro-1H-indene-2-carboxylate (4d)

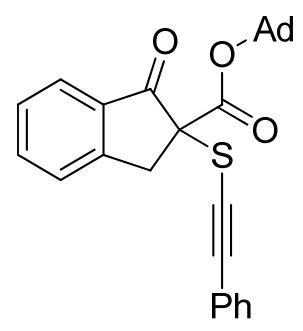

Yield:33.6 mg (76\%); time: 3.0 h; brown liquid; TLC, $\mathrm{R}_{\mathrm{f}}=0.36(\mathrm{PE}: \mathrm{EtOAc}=9: 1) ;{ }^{1} \mathrm{H} \mathrm{NMR}\left(\mathrm{CDCl}_{3}, 400\right.$ MHz): $\delta 7.85(\mathrm{~d}, 1 \mathrm{H}, \mathrm{J}=7.6 \mathrm{~Hz}), 7.65(\mathrm{td}, 1 \mathrm{H}, \mathrm{J}=7.6,1.2 \mathrm{~Hz}), 7.48(\mathrm{~d}, 1 \mathrm{H}, \mathrm{J}=7.6 \mathrm{~Hz}), 7.43(\mathrm{t}, 1 \mathrm{H}, \mathrm{J}=7.6$ Hz), 7.22-7.12 (m, 3H), 6.93-6.90 (m, 2H), 3.99 (d, 1H, J = 17.6 Hz), 3.73 (d, 1H, J = 18.0 Hz), 2.15 (s, 4H), 2.09 (d, 6H, J =2.8 Hz), $1.63(\mathrm{~s}, 6 \mathrm{H}) ;{ }^{13} \mathrm{C}\left\{{ }^{1} \mathrm{H}\right\} \mathrm{NMR}\left(\mathrm{CDCl}_{3}, 100 \mathrm{MHz}\right): \delta 197.7,166.0,152.1,135.7,134.6$, $130.9,128.1,128.03,127.98,125.9,125.2,122.6,97.2,84.5,75.6,64.7,41.0,40.2,35.9,30.9$; HRMS (ESI) $\mathrm{m} / \mathrm{z}$ calcd. for $\mathrm{C}_{28} \mathrm{H}_{27} \mathrm{O}_{3} \mathrm{~S}[\mathrm{M}+\mathrm{H}]^{+}: 443.1675$, found: 443.1667 .

Methyl 5-bromo-1-oxo-2-((phenylethynyl)thio)-2,3-dihydro-1H-indene-2-carboxylate (4e) 


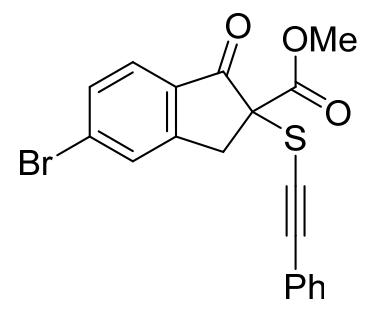

Yield:37.3 mg (93\%); time: 3.0 h; brown liquid; TLC, $\mathrm{R}_{\mathrm{f}}=0.38(\mathrm{PE}: \mathrm{EtOAc}=9: 1)$; ${ }^{1} \mathrm{H}$ NMR $\left(\mathrm{CDCl}_{3}, 400\right.$ MHz): $\delta 8.04(\mathrm{t}, 2 \mathrm{H}, \mathrm{J}=8.4 \mathrm{~Hz}), 7.92(\mathrm{~d}, 1 \mathrm{H}, \mathrm{J}=8.0 \mathrm{~Hz}), 7.60-7.55(\mathrm{~m}, 3 \mathrm{H}), 7.36(\mathrm{dd}, 2 \mathrm{H}, \mathrm{J}=8.0,1.6 \mathrm{~Hz})$, $4.39(\mathrm{~d}, 1 \mathrm{H}, \mathrm{J}=18.0 \mathrm{~Hz}), 4.17(\mathrm{~s}, 3 \mathrm{H}), 4.07(\mathrm{~d}, 1 \mathrm{H}, 18.0 \mathrm{~Hz}) ;{ }^{13} \mathrm{C}\left\{{ }^{1} \mathrm{H}\right\} \mathrm{NMR}\left(\mathrm{CDCl}_{3}, 100 \mathrm{MHz}\right): \delta 195.8$, $167.4,153.3,133.1,131.9,131.7,131.1,129.3,128.5,128.2,128.1,126.5,122.2,98.3,74.5,63.8,54.0,39.4$; HRMS (ESI) m/z calcd. for $\mathrm{C}_{19} \mathrm{H}_{14} \mathrm{BrO}_{3} \mathrm{~S}[\mathrm{M}+\mathrm{H}]^{+}$: 402.9820, 400.9842, found: 402.9822, 400.9834 .

Methyl 4-bromo-1-oxo-2-((phenylethynyl)thio)-2,3-dihydro-1H-indene-2-carboxylate (4f)<smiles>COC(=O)C1(SC#Cc2ccccc2)Cc2c(Br)cccc2C1=O</smiles>

Yield:24.1 mg (60\%); time: 3.0 h; brown liquid; TLC, $\mathrm{R}_{\mathrm{f}}=0.38(\mathrm{PE}: \mathrm{EtOAc}=9: 1) ;{ }^{1} \mathrm{H} \mathrm{NMR}\left(\mathrm{CDCl}_{3}, 400\right.$ MHz): $\delta 7.80(\mathrm{dd}, 2 \mathrm{H}, \mathrm{J}=7.6,2.4 \mathrm{~Hz}), 7.33(\mathrm{t}, 1 \mathrm{H}, \mathrm{J}=8.4 \mathrm{~Hz}), 7.25-7.18(\mathrm{~m}, 3 \mathrm{H}), 7.06(\mathrm{dd}, 2 \mathrm{H}, \mathrm{J}=8.0,1.2$ $\mathrm{Hz}), 3.97(\mathrm{~d}, 1 \mathrm{H}, \mathrm{J}=18.8 \mathrm{~Hz}), 3.84(\mathrm{~s}, 3 \mathrm{H}), 3.69(\mathrm{~d}, 1 \mathrm{H}, \mathrm{J}=18.4 \mathrm{~Hz}) ;{ }^{13} \mathrm{C}\left\{{ }^{1} \mathrm{H}\right\} \mathrm{NMR}\left(\mathrm{CDCl}_{3}, 100 \mathrm{MHz}\right): \delta$ $196.3,167.4,151.5,138.6,136.2,131.2,129.9,128.5,128.1,124.1,122.2,121.3,98.5,74.3,63.5,54.0,40.8$; HRMS (ESI) m/z calcd. for $\mathrm{C}_{19} \mathrm{H}_{13} \mathrm{BrO}_{3} \mathrm{~S}[\mathrm{M}+\mathrm{H}]^{+}$: 402.9822, 400.9842, found: 402.9806, 400.9825.

Methyl 6-methyl-1-oxo-2-((phenylethynyl)thio)-2,3-dihydro-1H-indene-2-carboxylate (4g)<smiles>COC(=O)C1(SC#Cc2ccccc2)Cc2ccc(C)cc2C1=O</smiles>

Yield:22.5 mg (67\%); time: 3.0 h; brown liquid; TLC, $\mathrm{R}_{\mathrm{f}}=0.37(\mathrm{PE}: \mathrm{EtOAc}=9: 1)$; ${ }^{1} \mathrm{H}$ NMR $\left(\mathrm{CDCl}_{3}, 400\right.$ MHz): $\delta 7.65(\mathrm{~s}, 1 \mathrm{H}), 7.48(\mathrm{~d}, 1 \mathrm{H}, \mathrm{J}=8.0 \mathrm{~Hz}), 7.38(\mathrm{~d}, 1 \mathrm{H}, \mathrm{J}=8.0 \mathrm{~Hz}), 7.23-7.16(\mathrm{~m}, 3 \mathrm{H}), 7.00(\mathrm{~d}, 2 \mathrm{H}, \mathrm{J}=$ $6.8 \mathrm{~Hz}), 4.01(\mathrm{~d}, 1 \mathrm{H}, \mathrm{J}=17.6 \mathrm{~Hz}), 3.81(\mathrm{~s}, 3 \mathrm{H}), 3.70(\mathrm{~d}, 1 \mathrm{H}, \mathrm{J}=17.6 \mathrm{~Hz}), 2.42(\mathrm{~s}, 3 \mathrm{H}) ;{ }^{13} \mathrm{C}\left\{{ }^{1} \mathrm{H}\right\} \mathrm{NMR}\left(\mathrm{CDCl}_{3}\right.$, $100 \mathrm{MHz}): \delta$ 196.8, 168.0, 149.3, 138.3, 137.3, 134.4, 131.1, 128.3, 128.1, 125.7, 125.2, 122.5, 97.8, 75.0, 64.2, 53.8, 39.6, 21.0; HRMS (ESI) m/z calcd. for $\mathrm{C}_{20} \mathrm{H}_{17} \mathrm{O}_{3} \mathrm{~S}[\mathrm{M}+\mathrm{H}]^{+}: 337.0893$, found: 337.0897 


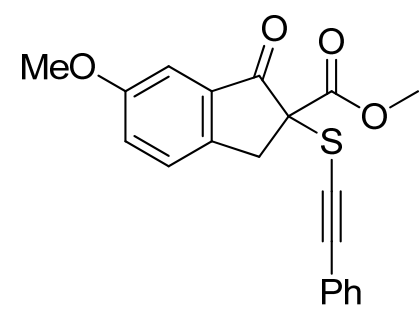

Yield: $32.1 \mathrm{mg}$ (91\%); time: $3.0 \mathrm{~h}$; brown liquid; TLC, Rf = $0.37(\mathrm{PE}: \mathrm{EtOAc}=9: 1) ;{ }^{1} \mathrm{H} \mathrm{NMR}\left(\mathrm{CDCl}_{3}, 400\right.$ MHz): $\delta$ 7.39-7.37 (m, 1H), 7.28-7.17 (m, 5H), 7.05-7.02 (m, 2H), $3.97(\mathrm{~d}, 1 \mathrm{H}, \mathrm{J}=18.0 \mathrm{~Hz}), 3.83(\mathrm{~d}, 6 \mathrm{H}, \mathrm{J}=$ 8.4 Hz); $3.66(\mathrm{~d}, 1 \mathrm{H}, \mathrm{J}=17.6 \mathrm{~Hz}) ;{ }^{13} \mathrm{C}\left\{{ }^{1} \mathrm{H}\right\} \mathrm{NMR}\left(\mathrm{CDCl}_{3}, 100 \mathrm{MHz}\right): \delta 196.8,167.9,159.9,144.8,135.4$, 131.0, 128.3, 128.1, 126.7, 125.7, 122.4, 106.1, 97.8, 74.8, 64.5, 55.7, 53.9, 39.3; HRMS (ESI) m/z calcd. for $\mathrm{C}_{20} \mathrm{H}_{17} \mathrm{O} 4 \mathrm{~S}[\mathrm{M}+\mathrm{H}]^{+}: 353.0842$, found: 353.0850

Methyl 1-oxo-2-((phenylethynyl)thio)-1,2,3,4-tetrahydronaphthalene-2-carboxylate (4i)<smiles>COC(=O)C1(SC#Cc2ccccc2)CCc2ccccc2C1=O</smiles>

Yield:21.8 mg (65\%); time: 3.0 h; brown liquid; TLC, $\mathrm{R}_{\mathrm{f}}=0.45(\mathrm{PE}: \mathrm{EtOAc}=9: 1) ;{ }^{1} \mathrm{H}$ NMR $\left(\mathrm{CDCl}_{3}, 400\right.$ MHz): $\delta 8.07(\mathrm{dd}, 1 \mathrm{H}, \mathrm{J}=7.6,0.8 \mathrm{~Hz}), 7.53(\mathrm{td}, 1 \mathrm{H}, \mathrm{J}=7.6,1.6 \mathrm{~Hz}), 7.43-7.41(\mathrm{~m}, 2 \mathrm{H}), 7.37-7.25(\mathrm{~m}, 5 \mathrm{H})$, $3.78(\mathrm{~s}, 3 \mathrm{H}), 3.22-3.13(\mathrm{~m}, 3 \mathrm{H}), 2.56-2.49(\mathrm{~m}, 1 \mathrm{H}) ;{ }^{13} \mathrm{C}\left\{{ }^{1} \mathrm{H}\right\} \mathrm{NMR}\left(\mathrm{CDCl}_{3}, 100 \mathrm{MHz}\right): \delta 189.9,167.7,143.1$, $134.4,131.5,130.9,128.9,128.44,128.39,128.2,127.1,122.9,98.5,75.2,64.5,53.5,32.7,26.6$; HRMS (ESI) m/z calcd. for $\mathrm{C}_{20} \mathrm{H}_{17} \mathrm{O}_{3} \mathrm{~S}[\mathrm{M}+\mathrm{H}]^{+}: 337.0893$, found: 337.0898 .

tert-Butyl 2-oxo-1-((phenylethynyl)thio)cyclopentane-1-carboxylate (4j)<smiles>CC(C)(C)OC(=O)C1(SC#Cc2ccccc2)CCCC1=O</smiles>

Yield:13.9 mg (44\%); time: 3.0 h; brown liquid; TLC, $\mathrm{R}_{\mathrm{f}}=0.40(\mathrm{PE}: \mathrm{EtOAc}=9: 1) ;{ }^{1} \mathrm{H} \mathrm{NMR}\left(\mathrm{CDCl}_{3}, 400\right.$ MHz): $\delta 7.42-7.40(\mathrm{~m}, 2 \mathrm{H}), 7.32-7.28(\mathrm{~m}, 3 \mathrm{H}), 2.77-2.70(\mathrm{~m}, 1 \mathrm{H}), 2.56-2.38(\mathrm{~m}, 3 \mathrm{H}), 2.20-2.03(\mathrm{~m}, 2 \mathrm{H}), 1.48$ (s, 9H); ${ }^{13} \mathrm{C}\left\{{ }^{1} \mathrm{H}\right\} \mathrm{NMR}\left(\mathrm{CDCl}_{3}, 100 \mathrm{MHz}\right): \delta 207.8,166.9,131.6,128.5,128.3,122.8,96.5,84.0,75.9,65.0$, 37.4, 35.1, 27.8, 19.7; HRMS (ESI) m/z calcd. for $\mathrm{C}_{18} \mathrm{H}_{21} \mathrm{O}_{3} \mathrm{~S}[\mathrm{M}+\mathrm{H}]^{+}: 334.1471$, found: 334.1471. 


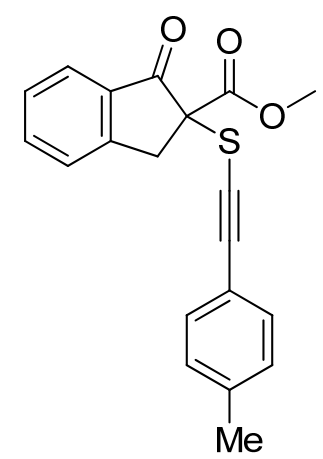

Yield: $30.3 \mathrm{mg}$ (90\%); time: $3.0 \mathrm{~h}$; brown liquid; TLC, $\mathrm{R}_{\mathrm{f}}=0.38(\mathrm{PE}: \mathrm{EtOAc}=9: 1) ;{ }^{1} \mathrm{H} \mathrm{NMR}\left(\mathrm{CDCl}_{3}, 400\right.$ MHz): $\delta 7.78(\mathrm{~d}, 1 \mathrm{H}, \mathrm{J}=7.6 \mathrm{~Hz}), 7.59(\mathrm{t}, 1 \mathrm{H}, \mathrm{J}=7.6 \mathrm{~Hz}), 7.42-7.35(\mathrm{~m}, 2 \mathrm{H}), 6.91(\mathrm{~d}, 2 \mathrm{H}, \mathrm{J}=8.0 \mathrm{~Hz}), 6.82$ $(\mathrm{d}, 2 \mathrm{H}, \mathrm{J}=8.0 \mathrm{~Hz}), 3.98(\mathrm{~d}, 1 \mathrm{H}, \mathrm{J}=18.0 \mathrm{~Hz}), 3.75(\mathrm{~s}, 3 \mathrm{H}), 3.68(\mathrm{~d}, 1 \mathrm{H}, \mathrm{J}=17.6 \mathrm{~Hz}), 2.21(\mathrm{~s}, 3 \mathrm{H}) ;{ }^{13} \mathrm{C}\left\{{ }^{1} \mathrm{H}\right\}$ $\mathrm{NMR}\left(\mathrm{CDCl}_{3}, 100 \mathrm{MHz}\right): \delta 197.0,167.9,151.9,138.6,136.0,134.2,131.1,128.8,128.2,126.0,125.4,119.3$, 98.1, 73.8, 63.8, 53.9, 39.9, 21.4; HRMS (ESI) m/z calcd. for $\mathrm{C}_{20} \mathrm{H}_{17} \mathrm{O}_{3} \mathrm{~S}[\mathrm{M}+\mathrm{H}]^{+}:$337.0893, found: 337.0880 .

\section{Methyl 2-(((4-fluorophenyl)ethynyl)thio)-1-oxo-2,3-dihydro-1H-indene-2-carboxylate (4m)}

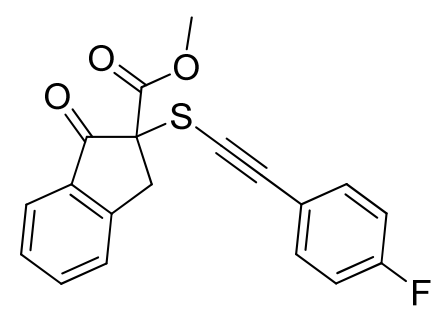

Yield:26.5 mg (78\%); time: 3.0 h; brown liquid; TLC, $\mathrm{R}_{\mathrm{f}}=0.35(\mathrm{PE}: \mathrm{EtOAc}=9: 1)$; ${ }^{1} \mathrm{H}$ NMR $\left(\mathrm{CDCl}_{3}, 400\right.$ MHz): $\delta 7.85(\mathrm{~d}, 1 \mathrm{H}, \mathrm{J}=7.6 \mathrm{~Hz}), 7.66(\mathrm{t}, 1 \mathrm{H}, \mathrm{J}=7.6 \mathrm{~Hz}), 7.49-7.42(\mathrm{~m}, 2 \mathrm{H}), 7.00-6.96(\mathrm{~m}, 2 \mathrm{H}), 6.89-6.85$ $(\mathrm{m}, 2 \mathrm{H}), 4.05(\mathrm{~d}, 1 \mathrm{H}, \mathrm{J}=18.0 \mathrm{~Hz}), 3.82(\mathrm{~s}, 3 \mathrm{H}), 3.72(\mathrm{~d}, 1 \mathrm{H}, \mathrm{J}=17.6 \mathrm{~Hz}) ;{ }^{13} \mathrm{C}\left\{{ }^{1} \mathrm{H}\right\} \mathrm{NMR}\left(\mathrm{CDCl}_{3}, 100 \mathrm{MHz}\right)$ : $\delta 196.8,167.9,162.5(\mathrm{~J}=248.0 \mathrm{~Hz}), 151.8,136.0,134.2,133.2(\mathrm{~J}=8.0 \mathrm{~Hz}), 128.2,126.0,125.4,115.4(\mathrm{~J}=$ $22.0 \mathrm{~Hz}$ ), 96.8, 74.5, 63.6, 53.9, 39.9; HRMS (ESI) m/z calcd. for $\mathrm{C}_{19} \mathrm{H}_{14} \mathrm{FO}_{3} \mathrm{~S}[\mathrm{M}+\mathrm{H}]^{+}:$341.0642, found: 341.0646.

\section{Methyl 2-(((3-fluorophenyl)ethynyl)thio)-1-oxo-2,3-dihydro-1H-indene-2-carboxylate (4n)}<smiles>COC(=O)C1(SC#Cc2cccc(F)c2)Cc2ccccc2C1=O</smiles>

Yield:33.4 mg (98\%); time: 3.0 h; brown liquid; TLC, $\mathrm{R}_{\mathrm{f}}=0.35(\mathrm{PE}: \mathrm{EtOAc}=9: 1)$; ${ }^{1} \mathrm{H}$ NMR $\left(\mathrm{CDCl}_{3}, 400\right.$ MHz): $\delta 7.87(\mathrm{~d}, 1 \mathrm{H}, \mathrm{J}=7.6 \mathrm{~Hz}), 7.70(\mathrm{t}, 1 \mathrm{H}, \mathrm{J}=8.4 \mathrm{~Hz}), 7.52-7.45(\mathrm{~m}, 2 \mathrm{H}), 7.16-7.10(\mathrm{~m}, 1 \mathrm{H}), 6.94-6.89$ 
$(\mathrm{m}, 1 \mathrm{H}), 6.75(\mathrm{~d}, 1 \mathrm{H}, \mathrm{J}=7.6 \mathrm{~Hz}), 6.57(\mathrm{~d}, 1 \mathrm{H}, \mathrm{J}=9.2 \mathrm{~Hz}), 4.07(\mathrm{~d}, 1 \mathrm{H}, \mathrm{J}=18.0 \mathrm{~Hz}), 3.82(\mathrm{~s}, 3 \mathrm{H}), 3.72(\mathrm{~d}$, $1 \mathrm{H}, \mathrm{J}=18.0 \mathrm{~Hz}) ;{ }^{13} \mathrm{C}\left\{{ }^{1} \mathrm{H}\right\} \mathrm{NMR}\left(\mathrm{CDCl}_{3}, 100 \mathrm{MHz}\right): \delta 196.8,167.8,162.0(\mathrm{~J}=245.0 \mathrm{~Hz}), 151.8,136.2,134.2$, $129.7(\mathrm{~J}=9.0 \mathrm{~Hz}), 128.3,126.6,126.0,125.4,124.2,124.1(\mathrm{~J}=9.0 \mathrm{~Hz}), 117.5(\mathrm{~J}=23.0 \mathrm{~Hz}), 155.5(\mathrm{~J}=21.0$ $\mathrm{Hz}), 96.6,76.3,63.7,53.9,40.0$; HRMS (ESI) m/z calcd. for $\mathrm{C}_{19} \mathrm{H}_{14} \mathrm{FO}_{3} \mathrm{~S}[\mathrm{M}+\mathrm{H}]^{+}:$341.0642, found: 341.0558 .

\section{Methyl 2-((naphthalen-1-ylethynyl)thio)-1-oxo-2,3-dihydro-1H-indene-2-carboxylate (40)}

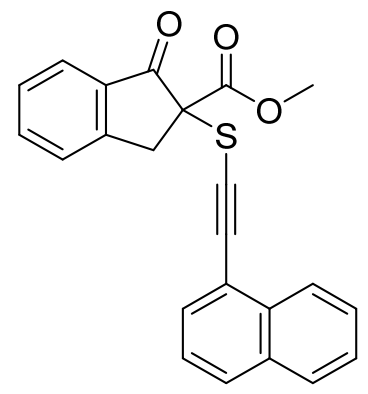

Yield: $36.1 \mathrm{mg}$ (97\%); time: $3.0 \mathrm{~h}$; brown liquid; TLC, $\mathrm{R}_{\mathrm{f}}=0.30(\mathrm{PE}: \mathrm{EtOAc}=9: 1) ;{ }^{1} \mathrm{H} \mathrm{NMR}\left(\mathrm{CDCl}_{3}, 400\right.$ MHz): $\delta 7.88(\mathrm{~d}, 1 \mathrm{H}, \mathrm{J}=7.6 \mathrm{~Hz}), 7.79-7.71(\mathrm{~m}, 3 \mathrm{H}), 7.63(\mathrm{t}, 1 \mathrm{H}, \mathrm{J}=7.2 \mathrm{~Hz}), 7.48-7.35(\mathrm{~m}, 4 \mathrm{H}), 7.30-7.25$ $(\mathrm{m}, 2 \mathrm{H}), 4.10(\mathrm{~d}, 1 \mathrm{H}, \mathrm{J}=18.0 \mathrm{~Hz}), 3.83(\mathrm{t}, 4 \mathrm{H}, \mathrm{J}=10.4 \mathrm{~Hz}) ;{ }^{13} \mathrm{C}\left\{{ }^{1} \mathrm{H}\right\} \mathrm{NMR}\left(\mathrm{CDCl}_{3}, 100 \mathrm{MHz}\right): \delta 196.8$, $167.9,151.7,136.1,134.1,132.9,130.3,128.8,128.3,128.1,126.7,126.3,126.1,125.8,125.4,124.9,120.1$, 96.1, 79.4, 63.9, 53.9, 39.9; HRMS (ESI) m/z calcd. for $\mathrm{C}_{23} \mathrm{H}_{17} \mathrm{O}_{3} \mathrm{~S}[\mathrm{M}+\mathrm{H}]^{+}:$373.0893, found: 373.0897.

\section{Methyl 2-((3,3-dimethylbut-1-yn-1-yl)thio)-1-oxo-2,3-dihydro-1H-indene-2-carboxylate (4p)}

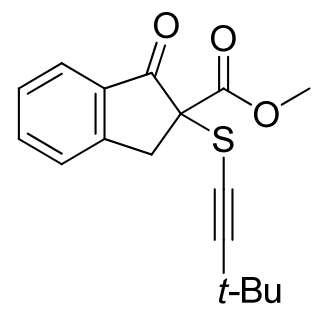

Yield: $28.7 \mathrm{mg}$ (95\%); time: $3.0 \mathrm{~h}$; brown liquid; TLC, $\mathrm{R}_{\mathrm{f}}=0.35(\mathrm{PE}: \mathrm{EtOAc}=9: 1) ;{ }^{1} \mathrm{H} \mathrm{NMR}\left(\mathrm{CDCl}_{3}, 400\right.$ MHz): $\delta 7.82(\mathrm{~d}, 1 \mathrm{H}, \mathrm{J}=8.0 \mathrm{~Hz}), 7.66(\mathrm{t}, 1 \mathrm{H}, \mathrm{J}=8.0 \mathrm{~Hz}), 7.49(\mathrm{~d}, 1 \mathrm{H}, \mathrm{J}=7.6 \mathrm{~Hz}), 7.42(\mathrm{t}, 1 \mathrm{H}, \mathrm{J}=7.6 \mathrm{~Hz})$, $4.00(\mathrm{~d}, 1 \mathrm{H}, \mathrm{J}=18.0 \mathrm{~Hz}), 3.77(\mathrm{~s}, 3 \mathrm{H}), 3.69$ (d, 1H, J = 18.0 Hz), $0.86(\mathrm{~s}, 9 \mathrm{H}) ;{ }^{13} \mathrm{C}\left\{{ }^{1} \mathrm{H}\right\} \mathrm{NMR}\left(\mathrm{CDCl}_{3}, 100\right.$ $\mathrm{MHz}): \delta 197.6,168.0,152.3,135.9,134.6,128.1,125.8,125.1,107.2,63.4,62.5,53.7,40.0,30.2,28.5$; HRMS (ESI) m/z calcd. for $\mathrm{C}_{17} \mathrm{H}_{19} \mathrm{O}_{3} \mathrm{~S}[\mathrm{M}+\mathrm{H}]^{+}: 303.1049$, found: 303.1057

Methyl 2-(hex-1-yn-1-ylthio)-1-oxo-2,3-dihydro-1H-indene-2-carboxylate (4q) 


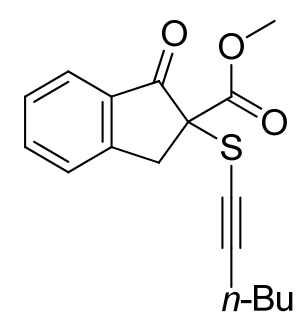

Yield: 24.5mg (81\%); time: $3.0 \mathrm{~h}$; brown liquid; TLC, $\mathrm{R}_{\mathrm{f}}=0.35(\mathrm{PE}: \mathrm{EtOAc}=9: 1) ;{ }^{1} \mathrm{H} \mathrm{NMR}\left(\mathrm{CDCl}_{3}, 400\right.$ MHz): $\delta 7.82(\mathrm{~d}, 1 \mathrm{H}, \mathrm{J}=7.6 \mathrm{~Hz}), 7.66(\mathrm{t}, 1 \mathrm{H}, \mathrm{J}=14.8 \mathrm{~Hz}), 7.48(\mathrm{~d}, 1 \mathrm{H}, \mathrm{J}=7.6 \mathrm{~Hz}), 7.42(\mathrm{t}, 1 \mathrm{H}, \mathrm{J}=7.6 \mathrm{~Hz})$, $3.99(\mathrm{~d}, 1 \mathrm{H}, \mathrm{J}=17.6 \mathrm{~Hz}), 3.78(\mathrm{~s}, 3 \mathrm{H}), 3.66(\mathrm{~d}, 1 \mathrm{H}, \mathrm{J}=18.0 \mathrm{~Hz}), 2.06-2.03(\mathrm{~m}, 2 \mathrm{H}), 1.17-1.08(\mathrm{~m}, 4 \mathrm{H}), 0.75$ $(\mathrm{t}, 3 \mathrm{H}, \mathrm{J}=6.8 \mathrm{~Hz}) ;{ }^{13} \mathrm{C}\left\{{ }^{1} \mathrm{H}\right\} \mathrm{NMR}\left(\mathrm{CDCl}_{3}, 100 \mathrm{MHz}\right): \delta 197.2,168.1,152.0,135.9,134.3,128.1,125.9,125.2$, 99.8, 63.8, 63.3, 53.7, 39.8, 30.2, 21.6, 19.6, 13.5; HRMS (ESI) m/z calcd. for $\mathrm{C}_{17} \mathrm{H}_{19} \mathrm{O}_{3} \mathrm{~S}[\mathrm{M}+\mathrm{H}]^{+}: 303.1049$, found: 303.1047 .

\section{General Procedure for Cu-Catalyzed Alkynylthiolation of Boronic Acids}

p-Tolylboronic acid $(0.1 \mathrm{mmol}), N$-phenylethynylthio phthalimide $3 \mathbf{a}(0.15 \mathrm{mmol}), \mathrm{K}_{2} \mathrm{CO}_{3}(0.2 \mathrm{mmol}), \mathrm{CuI}$ $(0.02 \mathrm{mmol})$, and 2,2-bipyridine $(0.02 \mathrm{mmol})$ in THF $(1 \mathrm{~mL})$ were placed into an oven-dried flask that was equipped with a stirring bar under nitrogen. The reaction was was stirred at $60{ }^{\circ} \mathrm{C}$ for $2 \mathrm{~h}$. The solvent was removed under vacuum, and the residue was purified by column chromatography $(\mathrm{PE} / \mathrm{EtOAc}=20: 1)$ to give 5a in $96 \%$ yield.

\section{(Phenylethynyl)(p-tolyl)sulfane (5a) ${ }^{4}$}

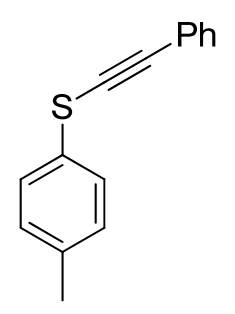

Yield:21.5 mg (96\%); time: $3.0 \mathrm{~h}$; brown liquid; TLC, $\mathrm{R}_{\mathrm{f}}=0.38(\mathrm{PE}) ;{ }^{1} \mathrm{H}$ NMR $\left(400 \mathrm{MHz}, \mathrm{CDCl}_{3}\right): \delta 7.52-$ $7.50(\mathrm{~m}, 2 \mathrm{H}), 7.40(\mathrm{~d}, 2 \mathrm{H}, \mathrm{J}=8.0 \mathrm{~Hz}), 7.34(\mathrm{t}, 3 \mathrm{H}, \mathrm{J}=3.2 \mathrm{~Hz}), 7.18(\mathrm{~d}, 2 \mathrm{H}, \mathrm{J}=8.0 \mathrm{~Hz}), 2.35(\mathrm{~s}, 3 \mathrm{H}) ;{ }^{13} \mathrm{C}\left\{{ }^{1} \mathrm{H}\right\}$ NMR ( $\left.\mathrm{CDCl}_{3}, 100 \mathrm{MHz}\right): \delta 136.6,131.7,130.0,129.8,129.2,128.5,128.4,126.5,123.0,97.2,76.1,21.0$.

\section{Phenyl(phenylethynyl)sulfane (5b) ${ }^{4}$}

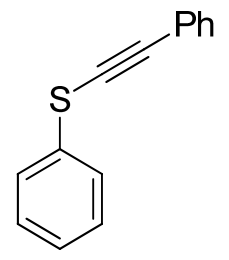


Yield:18.3 mg (87\%); time: 3.0 h; brown liquid; TLC, Rf = $0.43(\mathrm{PE}) ;{ }^{1} \mathrm{H}$ NMR $\left(400 \mathrm{MHz}, \mathrm{CDCl}_{3}\right): \delta$ 7.44$7.39(\mathrm{~m}, 4 \mathrm{H}), 7.26-7.24(\mathrm{~m}, 5 \mathrm{H}), 7.16-7.14(\mathrm{~m}, 1 \mathrm{H}) ;{ }^{13} \mathrm{C}\left\{{ }^{1} \mathrm{H}\right\} \mathrm{NMR}\left(\mathrm{CDCl}_{3}, 100 \mathrm{MHz}\right): \delta 132.9,131.7,131.6$, $131.0,129.2,129.2,128.6,128.4,128.3,126.5,126.2,122.9,97.9,75.4$.

(4-Chlorophenyl)(phenylethynyl)sulfane (5c) ${ }^{4}$

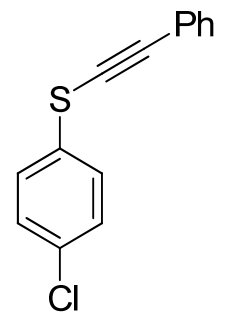

Yield:17.6 mg (72\%); time: $3.0 \mathrm{~h}$; colorless liquid; TLC, $\mathrm{R}_{\mathrm{f}}=0.46(\mathrm{PE}) ;{ }^{1} \mathrm{H}$ NMR $\left(400 \mathrm{MHz}, \mathrm{CDCl}_{3}\right): \delta 7.52-$ $7.50(\mathrm{~m}, 2 \mathrm{H}), 7.41(\mathrm{~d}, 2 \mathrm{H}, \mathrm{J}=8.4 \mathrm{~Hz}), 7.36-7.32(\mathrm{~m}, 5 \mathrm{H}) ;{ }^{13} \mathrm{C}\left\{{ }^{1} \mathrm{H}\right\} \mathrm{NMR}\left(\mathrm{CDCl}_{3}, 100 \mathrm{MHz}\right): \delta 132.5,131.8$, $131.6,129.4,128.9,128.4,127.4,122.6,98.4,74.7$.

\section{(3-Chlorophenyl)(phenylethynyl)sulfane (5d)}

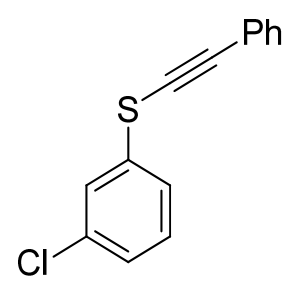

Yield: $16.9 \mathrm{mg}$ (69\%); time: $3 \mathrm{~h}$; colorless liquid; TLC, Rf=0.52 (PE); ${ }^{1} \mathrm{H}$ NMR $\left(400 \mathrm{MHz}, \mathrm{CDCl}_{3}\right): \delta 7.53$ $7.50(\mathrm{~m}, 2 \mathrm{H}), 7.47(\mathrm{t}, 1 \mathrm{H}, \mathrm{J}=2.0 \mathrm{~Hz}), 7.36-7.32(\mathrm{~m}, 4 \mathrm{H}), 7.26(\mathrm{t}, 1 \mathrm{H}, \mathrm{J}=7.6 \mathrm{~Hz}), 7.19(\mathrm{~d}, 1 \mathrm{H}, \mathrm{J}=8.0 \mathrm{~Hz})$; ${ }^{13} \mathrm{C}\left\{{ }^{1} \mathrm{H}\right\} \mathrm{NMR}\left(\mathrm{CDCl}_{3}, 100 \mathrm{MHz}\right): \delta 135.2,135.1,131.8,130.2,128.9,128.4,126.7,125.8,124.1,122.5$, 99.0, 74.1. HRMS (EI) m/z calcd. for $\mathrm{C}_{14} \mathrm{H}_{9} \mathrm{ClS}[\mathrm{M}]^{+}: 244.0108$, found: 244.0106 .

\section{(4-Bromophenyl)(phenylethynyl)sulfane (5e) ${ }^{4}$}

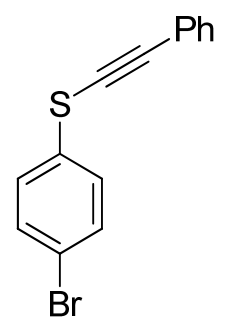

Yield: $15.6 \mathrm{mg}$ (54\%); time: $3 \mathrm{~h}$; colorless liquid; TLC, $\mathrm{R}_{\mathrm{f}}=0.56(\mathrm{PE}) ;{ }^{1} \mathrm{H}$ NMR $\left(\mathrm{CDCl}_{3}, 400 \mathrm{MHz}\right): \delta 7.52-$ $7.51(\mathrm{~m}, 2 \mathrm{H}), 7.47$ (d, 2H, J = 8.8 Hz), 7.36-7.34 (m, 5H); ${ }^{13} \mathrm{C}\left\{{ }^{1} \mathrm{H}\right\} \mathrm{NMR}\left(\mathrm{CDCl}_{3}, 100 \mathrm{MHz}\right): \delta 132.3,131.8$, $128.9,128.4,127.7,122.6,120.3,98.5,74.6$.

\section{3-((Phenylethynyl)thio)thiophene (5f)}




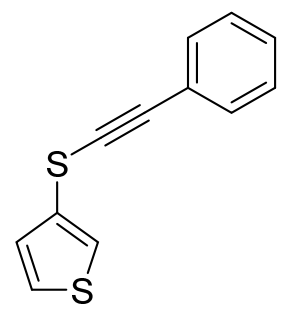

Yield:16.2 mg (75\%); time: 2.0 h; brown liquid; TLC, $\mathrm{R}_{\mathrm{f}}=0.47(\mathrm{PE}) ;{ }^{1} \mathrm{H} \mathrm{NMR}\left(\mathrm{CDCl}_{3}, 400 \mathrm{MHz}\right): \delta 7.49$ 7.46 (m, 2H), 7.40-7.39 (m, 1H), 7.34-7.33 (m, 4H), 7.16-7.15 (m, 1H); ${ }^{13} \mathrm{C}\left\{{ }^{1} \mathrm{H}\right\} \mathrm{NMR}\left(\mathrm{CDCl}_{3}, 100 \mathrm{MHz}\right): \delta$ 131.6, 128.5, 128.3, 127.4, 127.0, 122.8, 121.9, 95.8, 76.3; HRMS (ESI) m/z calcd. for $\mathrm{C}_{12} \mathrm{H}_{9} \mathrm{~S}_{2}[\mathrm{M}+\mathrm{H}]^{+}$: 217.0140, found: 217.0140 .

\section{Naphthalen-1-yl(phenylethynyl)sulfane (5g) ${ }^{5}$}

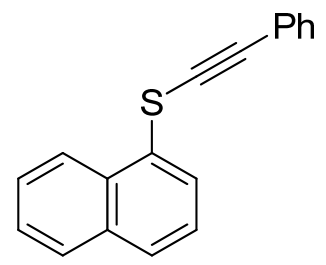

Yield: $14.3 \mathrm{mg}$ (55\%); time: $3 \mathrm{~h}$; colorless liquid; TLC, $\mathrm{R}_{\mathrm{f}}=0.33(\mathrm{PE}) ;{ }^{1} \mathrm{H}$ NMR $\left(\mathrm{CDCl}_{3}, 400 \mathrm{MHz}\right): \delta 8.13$ $(\mathrm{d}, 1 \mathrm{H}, \mathrm{J}=8.0 \mathrm{~Hz}), 7.93(\mathrm{dd}, 1 \mathrm{H}, \mathrm{J}=7.6,1.2 \mathrm{~Hz}), 7.88(\mathrm{~d}, 1 \mathrm{H} \mathrm{J}=8.8 \mathrm{~Hz}), 7.77(\mathrm{~d}, 1 \mathrm{H}, \mathrm{J}=8.0 \mathrm{~Hz}), 7.61-7.51$ $(\mathrm{m}, 5 \mathrm{H}), 7.35(\mathrm{~d}, 3 \mathrm{H}, \mathrm{J}=2.8 \mathrm{~Hz}) ;{ }^{13} \mathrm{C}\left\{{ }^{1} \mathrm{H}\right\} \mathrm{NMR}\left(\mathrm{CDCl}_{3}, 100 \mathrm{MHz}\right): \delta 133.88,131.8,130.7,129.7,128.7$, $128.6,128.4,127.5,126.6,126.5,125.9,125.5,123.4,122.9,97.8,75.5$.

\section{3-((p-Tolylthio)ethynyl)thiophene (5h)}<smiles>Cc1ccc(SC#Cc2ccsc2)cc1</smiles>

Yield: $19.1 \mathrm{mg}$ (83\%); time: $3 \mathrm{~h}$; brown liquid; TLC, $\mathrm{R}_{\mathrm{f}}=0.38(\mathrm{PE}) ;{ }^{1} \mathrm{H} \mathrm{NMR}\left(\mathrm{CDCl}_{3}, 400 \mathrm{MHz}\right): \delta 7.47$ (d, $1 \mathrm{H}, \mathrm{J}=2.0 \mathrm{~Hz}), 7.29(\mathrm{~d}, 2 \mathrm{H}, \mathrm{J}=8.0 \mathrm{~Hz}), 7.22-7.20(\mathrm{~m}, 1 \mathrm{H}), 7.09(\mathrm{t}, 3 \mathrm{H}, \mathrm{J}=5.6 \mathrm{~Hz}), 2.26(\mathrm{~s}, 3 \mathrm{H}) ;{ }^{13} \mathrm{C}\left\{{ }^{1} \mathrm{H}\right\}$ NMR (CDCl $3,100 \mathrm{MHz}): \delta 136.6,131.4,130.1,130.0,129.9,129.2,129.1,126.6,125.3,122.1,92.1,75.6$, 21.0; HRMS (ESI) m/z calcd. for $\mathrm{C}_{13} \mathrm{H}_{11} \mathrm{~S}_{2}[\mathrm{M}+\mathrm{H}]^{+}: 231.0297$, found: 231.0298 .

\section{(3,3-dimethylbut-1-yn-1-yl)(p-tolyl)sulfane (5i)}

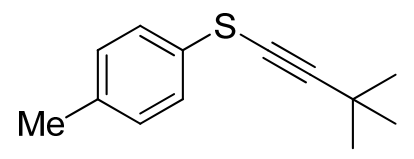

Yield: $15.9 \mathrm{mg}$ (78\%); time: $3 \mathrm{~h}$; brown liquid; TLC, $\mathrm{R}_{\mathrm{f}}=0.55(\mathrm{PE}) ;{ }^{1} \mathrm{H} \mathrm{NMR}\left(\mathrm{CDCl}_{3}, 400 \mathrm{MHz}\right): \delta 7.28(\mathrm{~d}$, $2 \mathrm{H}, \mathrm{J}=8.0 \mathrm{~Hz}), 7.13(\mathrm{~d}, 2 \mathrm{H}, \mathrm{J}=8.0 \mathrm{~Hz}), 2.32(\mathrm{~s}, 3 \mathrm{H}), 1.31(\mathrm{~s}, 9 \mathrm{H}) ;{ }^{13} \mathrm{C}\left\{{ }^{1} \mathrm{H}\right\} \mathrm{NMR}\left(\mathrm{CDCl}_{3}, 100 \mathrm{MHz}\right): \delta$ 
135.9, 130.1, 129. 8, 125.7, 107.3, 63.9, 30.9, 29.0, 20.9.; HRMS (ESI) m/z calcd. for $\mathrm{C}_{13} \mathrm{H}_{17} \mathrm{~S}[\mathrm{M}$ $+\mathrm{H}]^{+}: 205.1045$, found:205.1045.

\section{Hex-1-yn-1-yl(p-tolyl)sulfane (5j) ${ }^{6}$}<smiles>CCCCC#CSc1ccc(C)cc1</smiles>

Yield:13.7 mg (67\%); time: $3.0 \mathrm{~h}$; brown liquid; TLC, $\mathrm{R}_{\mathrm{f}}=0.58(\mathrm{PE}) ;{ }^{1} \mathrm{H} \mathrm{NMR}\left(\mathrm{CDCl}_{3}, 400 \mathrm{MHz}\right): \delta 7.30(\mathrm{~d}$, $2 \mathrm{H}, \mathrm{J}=8.4 \mathrm{~Hz}), 7.13(\mathrm{~d}, 2 \mathrm{H}, \mathrm{J}=8.0 \mathrm{~Hz}), 2.44$ (t, 2H, J = $7.2 \mathrm{~Hz}), 2.32(\mathrm{~s}, 3 \mathrm{H}), 1.61-1.54$ (m, 2H), 1.50-1.41 $(\mathrm{m}, 2 \mathrm{H}), 0.93(\mathrm{t}, 3 \mathrm{H}, \mathrm{J}=7.6 \mathrm{~Hz}) ;{ }^{13} \mathrm{C}\left\{{ }^{1} \mathrm{H}\right\} \mathrm{NMR}\left(\mathrm{CDCl}_{3}, 100 \mathrm{MHz}\right): \delta 136.1,130.1,129.8,126.1,99.3,65.1$, $30.7,22.0,20.0,13.6$.

\section{(4-Azidobut-1-yn-1-yl)(p-tolyl)sulfane (5k)}<smiles>Cc1ccc(SC#CCCN)cc1</smiles>

Yield:12.2mg (56\%); time: 3.0 h; brown liquid; TLC, $\mathrm{R}_{\mathrm{f}}=0.52(\mathrm{PE}) ;{ }^{1} \mathrm{H} \mathrm{NMR}\left(\mathrm{CDCl}_{3}, 400 \mathrm{MHz}\right): \delta 7.24(\mathrm{~d}$, $2 \mathrm{H}, \mathrm{J}=8.4 \mathrm{~Hz}), 7.07(\mathrm{~d}, 2 \mathrm{H}, \mathrm{J}=8.0 \mathrm{~Hz}), 3.40(\mathrm{t}, 2 \mathrm{H}, \mathrm{J}=6.8 \mathrm{~Hz}), 2.65(\mathrm{t}, 2 \mathrm{H}, \mathrm{J}=6.8 \mathrm{~Hz}), 2.26(\mathrm{~s}, 3 \mathrm{H}) ;{ }^{13} \mathrm{C}\left\{{ }^{1} \mathrm{H}\right\}$ NMR (CDCl $3,100 \mathrm{MHz}): \delta$ 136.6, 130.0, 129.0, 126.6, 94.4, 68.7, 49.9, 21.0; HRMS (ESI) m/z calcd. for $\mathrm{C}_{11} \mathrm{H}_{12} \mathrm{~N}_{3} \mathrm{~S}[\mathrm{M}+\mathrm{H}]^{+}:$218.0746, found: 218.0739 .

\section{General Procedure for Alkynylthiolation of Grignard Reagents}

In a $10 \mathrm{~mL}$ flask, $N$-phenylethynylthio phthalimide 3a $(0.15 \mathrm{mmol})$ was dissolved in THF $(1 \mathrm{~mL})$ and then the Grignard reagent solution $(0.5 \mathrm{M}$ in THF, $0.15 \mathrm{mmol})$ was added dropwise. After 2 hours the reaction was quenched with a saturated aqueous solution of $\mathrm{NH}_{4} \mathrm{Cl}$ and the product was extracted with EtOAc $(3 \times 15 \mathrm{~mL})$. The combined organic layers were dried over anhydrous $\mathrm{Na} 2 \mathrm{SO} 4$, filtered, and solvents were removed under vacuum. The residue was purified by flask column chromatography $(\mathrm{PE} / \mathrm{EtOAc}=20: 1)$ to afford $\mathbf{6 a}$ in $75 \%$ yield.

\section{(4-Methoxyphenyl)(phenylethynyl)sulfane (6a) ${ }^{5}$}<smiles>COc1ccc(SC#Cc2ccccc2)cc1</smiles> 
Yield: 36 mg (75\%); time: 1.0 h; brown liquid; TLC, $\mathrm{R}_{\mathrm{f}}=0.48(\mathrm{PE}) ;{ }^{1} \mathrm{H} \mathrm{NMR}\left(\mathrm{CDCl}_{3}, 400 \mathrm{MHz}\right): \delta 7.50-7.48$ $(\mathrm{m}, 2 \mathrm{H}), 7.44(\mathrm{~d}, 2 \mathrm{H}, \mathrm{J}=8.8 \mathrm{~Hz}), 7.33(\mathrm{t}, 3 \mathrm{H}, \mathrm{J}=3.2 \mathrm{~Hz}), 6.92(\mathrm{~d}, 2 \mathrm{H}, \mathrm{J}=8.8 \mathrm{~Hz}), 3.81(\mathrm{~s}, 3 \mathrm{H}) ;{ }^{13} \mathrm{C}\left\{{ }^{1} \mathrm{H}\right\}$ NMR (CDCl $3,100 \mathrm{MHz}): \delta 159.0,131.6,128.9,128.4,128.3,123.0,123.0,115.0,96.2,55.4$.

Hex-1-yn-1-yl(4-methoxyphenyl)sulfane (6b) ${ }^{6}$

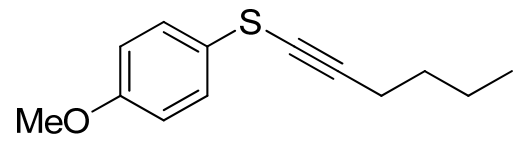

Yield:29.2 mg (88\%); time: $1.0 \mathrm{~h}$; brown liquid; TLC, $\mathrm{R}_{\mathrm{f}}=0.48(\mathrm{PE}) ;{ }^{1} \mathrm{H} \mathrm{NMR}\left(\mathrm{CDCl}_{3}, 400 \mathrm{MHz}\right): \delta 7.34(\mathrm{~d}$, $2 \mathrm{H}, \mathrm{J}=8.8 \mathrm{~Hz}), 6.88(\mathrm{~d}, 2 \mathrm{H}, \mathrm{J}=8.8 \mathrm{~Hz}), 3.80(\mathrm{~s}, 3 \mathrm{H}), 2.41(\mathrm{t}, 2 \mathrm{H}, \mathrm{J}=7.2 \mathrm{~Hz}), 1.60-1.53(\mathrm{~m}, 2 \mathrm{H}), 1.49-1.40$ (m, 2H), $0.93(\mathrm{t}, 3 \mathrm{H}, \mathrm{J}=7.2 \mathrm{~Hz}) ;{ }^{13} \mathrm{C}\left\{{ }^{1} \mathrm{H}\right\} \mathrm{NMR}\left(\mathrm{CDCl}_{3}, 100 \mathrm{MHz}\right): \delta 158.7,128.3,123.9,114.9,98.3,65.9$, $55.4,30.7,22.0,20.0,13.6$.

\section{(Cyclopropylethynyl)(4-methoxyphenyl)sulfane (6c)}

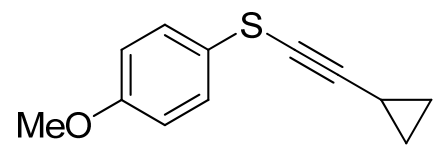

Yield:20.1 mg (66\%); time: 1.0 h; brown liquid; TLC, $\mathrm{R}_{\mathrm{f}}=0.45(\mathrm{PE}) ;{ }^{1} \mathrm{H} \mathrm{NMR}\left(\mathrm{CDCl}_{3}, 400 \mathrm{MHz}\right): \delta 7.33$ (dd, $2 \mathrm{H}, \mathrm{J}=6.8,2.0 \mathrm{~Hz}), 6.88(\mathrm{dd}, 2 \mathrm{H}, \mathrm{J}=6.8,2.0 \mathrm{~Hz}), 3.79(\mathrm{~s}, 3 \mathrm{H}), 1.48-1.42(\mathrm{~m}, 1 \mathrm{H}), 0.88-0.79(\mathrm{~m}, 4 \mathrm{H}) ;{ }^{13} \mathrm{C}\left\{{ }^{1} \mathrm{H}\right\}$ NMR $\left(\mathrm{CDCl}_{3}, 100 \mathrm{MHz}\right): \delta 158.7,128.2,124.0,114.9,61.8,55.4,9.1,0.9 ; \mathrm{HRMS}$ (ESI) m/z calcd. for $\mathrm{C}_{12} \mathrm{H}_{13} \mathrm{OS}[\mathrm{M}+\mathrm{H}]^{+}:$205.0682, found: 205.0686.

Triisopropyl(((4-methoxyphenyl)thio)ethynyl)silane (6d) ${ }^{5}$

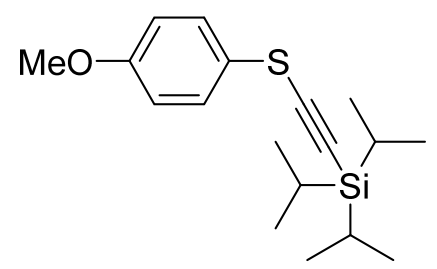

Yield:47.9 mg (98\%); time: $1.0 \mathrm{~h}$; brown liquid; TLC, $\mathrm{R}_{\mathrm{f}}=0.32(\mathrm{PE}) ;{ }^{1} \mathrm{H} \mathrm{NMR}\left(\mathrm{CDCl}_{3}, 400 \mathrm{MHz}\right): \delta 7.37$ $(\mathrm{d}, 2 \mathrm{H}, \mathrm{J}=8.8 \mathrm{~Hz}), 6.90(\mathrm{~d}, 2 \mathrm{H}, \mathrm{J}=8.8 \mathrm{~Hz}), 3.80(\mathrm{~s}, 3 \mathrm{H}), 1.11(\mathrm{~s}, 21 \mathrm{H}) ;{ }^{13} \mathrm{C}\left\{{ }^{1} \mathrm{H}\right\} \mathrm{NMR}\left(\mathrm{CDCl}_{3}, 100 \mathrm{MHz}\right): \delta$ $158.8,128.3,122.9,114.9,101.5,92.6,55.4,18.6,11.3$.

(Phenylethynyl)(p-tolylethynyl)sulfane (6e)

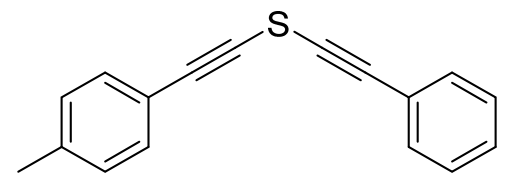

Yield: $21.8 \mathrm{mg}$ (59\%); time: $2.0 \mathrm{~h}$; brown liquid; TLC, $\mathrm{R}_{\mathrm{f}}=0.48(\mathrm{PE}) ;{ }^{1} \mathrm{H} \mathrm{NMR}\left(\mathrm{CDCl}_{3}, 400 \mathrm{MHz}\right): \delta 7.49$ (d, $2 \mathrm{H}, \mathrm{J}=9.2 \mathrm{~Hz}), 7.39(\mathrm{~d}, 2 \mathrm{H}, \mathrm{J}=8.4 \mathrm{~Hz}), 7.33(\mathrm{~d}, 3 \mathrm{H}, \mathrm{J}=6.8 \mathrm{~Hz}), 2.36(\mathrm{~s}, 3 \mathrm{H}) ;{ }^{13} \mathrm{C}\left\{{ }^{1} \mathrm{H}\right\} \mathrm{NMR}\left(\mathrm{CDCl}_{3}, 100\right.$ 
MHz): $\delta$ 139.4, 132.0, 131.9, 129.1, 128.3, 122.3, 119.1, 94.8, 94.5, 72.3, 71.0, 21.6; HRMS (ESI) m/z calcd. for $\mathrm{C}_{17} \mathrm{H}_{13} \mathrm{~S}[\mathrm{M}+\mathrm{H}]^{+}:$249.0732, found: 249.0748.

(Phenylethynyl)(prop-1-yn-1-yl)sulfane (6f) ${ }^{7}$<smiles>CC#CSC#Cc1ccccc1</smiles>

Yield:13.2mg (51\%); time: 2.0 h; brown liquid; TLC, $\mathrm{R}_{\mathrm{f}}=0.45(\mathrm{PE}) ;{ }^{1} \mathrm{H}$ NMR $\left(\mathrm{CDCl}_{3}, 400 \mathrm{MHz}\right): \delta 7.47-7.44$ (m, 2H), 7.34-7.31 (m, 3H), $2.00(\mathrm{~s}, 3 \mathrm{H}) ;{ }^{13} \mathrm{C}\left\{{ }^{1} \mathrm{H}\right\} \mathrm{NMR}\left(\mathrm{CDCl}_{3}, 100 \mathrm{MHz}\right): \delta 131.8,128.8,128.3,122.3$, $94.3,92.3,72.9,60.7,5.1$.

\section{(Phenylethynyl)(prop-1-en-2-yl)sulfane (6g) ${ }^{7}$}<smiles>C=C(C)SC#Cc1ccccc1</smiles>

Yield:19.8 mg (76 \%); time: 1.0 h; brown liquid; TLC, $\mathrm{R}_{\mathrm{f}}=0.52(\mathrm{PE}) ;{ }^{1} \mathrm{H} \mathrm{NMR}\left(\mathrm{CDCl}_{3}, 400 \mathrm{MHz}\right): \delta 7.40-$ $7.38(\mathrm{~m}, 2 \mathrm{H}), 7.25(\mathrm{t}, 3 \mathrm{H}, \mathrm{J}=2.8 \mathrm{~Hz}), 5.27(\mathrm{~s}, 1 \mathrm{H}), 5.15(\mathrm{~d}, 1 \mathrm{H}, \mathrm{J}=1.2 \mathrm{~Hz}), 2.00(\mathrm{~s}, 3 \mathrm{H}) ;{ }^{13} \mathrm{C}\left\{{ }^{1} \mathrm{H}\right\} \mathrm{NMR}$ $\left(\mathrm{CDCl}_{3}, 100 \mathrm{MHz}\right): \delta 136.7,131.5,128.5,128.3,123.0,98.6,75.7,21.8$.

3-((Prop-1-en-2-ylthio)ethynyl)thiophene (6h)

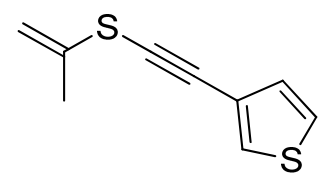

Yield:20.9 mg (77 \%); time: 1.0 h; brown liquid; TLC, $\mathrm{R}_{\mathrm{f}}=0.53(\mathrm{PE}) ;{ }^{1} \mathrm{H}$ NMR $\left(\mathrm{CDCl}_{3}, 400 \mathrm{MHz}\right): \delta 7.44$ (t, $1 \mathrm{H}, \mathrm{J}=2.0 \mathrm{~Hz}), 7.21-7.19(\mathrm{~m}, 1 \mathrm{H}), 7.06(\mathrm{~d}, 1 \mathrm{H}, \mathrm{J}=4.8 \mathrm{~Hz}), 5.24(\mathrm{~s}, 1 \mathrm{H}), 5.14(\mathrm{~s}, 1 \mathrm{H}), 2.00(\mathrm{~s}, 3 \mathrm{H}) ;{ }^{13} \mathrm{C}\left\{{ }^{1} \mathrm{H}\right\}$ NMR (CDCl 3,100 MHz): $\delta$ 136.8, 130.0, 129.7, 125.3, 122.0, 111.1, 93.5, 75.2, 21.8; HRMS (ESI) m/z calcd. for $\mathrm{C}_{17} \mathrm{H}_{13} \mathrm{~S}[\mathrm{M}+\mathrm{H}]^{+}:$181.0140, found: 181.0151 .

Hex-1-yn-1-yl(prop-1-en-2-yl)sulfane (6i)<smiles>C=C(C)SC#CCCCC</smiles>

Yield:18.1 mg (78 \%); time: $1.0 \mathrm{~h}$; brown liquid; TLC, $\mathrm{R}_{\mathrm{f}}=0.50(\mathrm{PE}) ;{ }^{1} \mathrm{H} \mathrm{NMR}\left(\mathrm{CDCl}_{3}, 400 \mathrm{MHz}\right): \delta 5.24(\mathrm{~s}$, $1 \mathrm{H}), 5.15(\mathrm{~d}, 1 \mathrm{H}, \mathrm{J}=1.2 \mathrm{~Hz}), 2.40(\mathrm{t}, 2 \mathrm{H}, \mathrm{J}=6.8 \mathrm{~Hz}), 1.99(\mathrm{~s}, 3 \mathrm{H}), 1.58-1.51(\mathrm{~m}, 2 \mathrm{H}), 1.48-1.39(\mathrm{~m}, 2 \mathrm{H})$, $0.92(\mathrm{t}, 3 \mathrm{H}, \mathrm{J}=7.2 \mathrm{~Hz}) ;{ }^{13} \mathrm{C}\left\{{ }^{1} \mathrm{H}\right\} \mathrm{NMR}\left(\mathrm{CDCl}_{3}, 100 \mathrm{MHz}\right): \delta 137.4,109.8,100.7,64.9,30.7,21.9,21.5,19.9$, 13.5; HRMS (ESI) m/z calcd. for $\mathrm{C}_{17} \mathrm{H}_{13} \mathrm{~S}[\mathrm{M}+\mathrm{H}]^{+}: 155.0889$, found: 155.0889 . 
<smiles>C=C(C)SC#CCCN</smiles>

Yield:17.8 mg (71 \%); time: $1.0 \mathrm{~h}$; brown liquid; TLC, $\mathrm{R}_{\mathrm{f}}=0.50(\mathrm{PE}) ;{ }^{1} \mathrm{H} \mathrm{NMR}\left(\mathrm{CDCl}_{3}, 400 \mathrm{MHz}\right): \delta 5.25(\mathrm{~s}$, 1H), $5.17(\mathrm{~d}, 1 \mathrm{H}, \mathrm{J}=1.6 \mathrm{~Hz}) ; 3.44(\mathrm{t}, 2 \mathrm{H}, \mathrm{J}=6.8 \mathrm{~Hz}) ; 2.69(\mathrm{t}, 2 \mathrm{H}, \mathrm{J}=6.8 \mathrm{~Hz}) ; 2.00(\mathrm{~s}, 3 \mathrm{H}) ;{ }^{13} \mathrm{C}\left\{{ }^{1} \mathrm{H}\right\} \mathrm{NMR}$ $\left(\mathrm{CDCl}_{3}, 100 \mathrm{MHz}\right): \delta 136.6,110.7,95.9,68.2,49.8,21.7,21.1$; HRMS (ESI) m/z calcd. for $\mathrm{C}_{7} \mathrm{H}_{10} \mathrm{~N}_{3} \mathrm{~S}$ $[\mathrm{M}+\mathrm{H}]^{+}: 167.0512$, found: 167.0504 .

\section{Ethyl(phenylethynyl)sulfane (6k) ${ }^{7}$}<smiles>CCSC#Cc1ccccc1</smiles>

Yield:18.7 mg (77\%); time: 1.0 h; brown liquid; TLC, $\mathrm{R}_{\mathrm{f}}=0.48(\mathrm{PE}) ;{ }^{1} \mathrm{H} \mathrm{NMR}\left(\mathrm{CDCl}_{3}, 400 \mathrm{MHz}\right): \delta 7.45$ $7.43(\mathrm{~m}, 2 \mathrm{H}), 7.32(\mathrm{t}, 3 \mathrm{H}, \mathrm{J}=4.0 \mathrm{~Hz}), 2.88-2.82(\mathrm{~m}, 2 \mathrm{H}), 1.50-1.47(\mathrm{~m}, 3 \mathrm{H}) ;{ }^{13} \mathrm{C}\left\{{ }^{1} \mathrm{H}\right\} \mathrm{NMR}\left(\mathrm{CDCl}_{3}, 100\right.$ MHz): $\delta 131.4,128.3,127.9,123.5,93.5,79.2,29.9$.

\section{Scheme S1 Reaction of Alcarazo Reagents with $\beta$-Keto Esters and Boronic acids}<smiles>COC(=O)C1Cc2ccccc2C1=O</smiles>

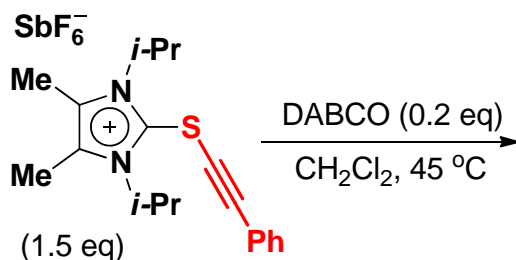<smiles>Oc1ccccc1</smiles><smiles>COC(=O)C1(C#Cc2ccccc2)Cc2ccccc2C1=O</smiles><smiles>COC(=O)C1(SC#Cc2ccccc2)Cc2ccccc2C1=O</smiles><smiles>CCCCC(C)OC(C)(C)C</smiles>

\section{Derivation of of Product 6b}

\section{Synthesis of $S$-(4-Methoxyphenyl) hexanethioate (7)}

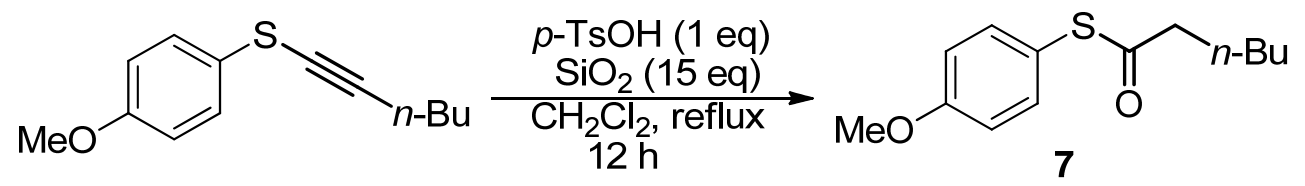

Hex-1-yn-1-yl(4-methoxyphenyl)sulfane (44 mg, $0.2 \mathrm{mmol})$ was dissolved in dry dichloromethane $(2 \mathrm{~mL})$ and p-toluenesulfonic acid (34 mg, $0.2 \mathrm{mmol}, 1.0$ equiv.) and $\mathrm{SiO}_{2}(400 \mathrm{mg}, 3.0 \mathrm{mmol}, 15$ equiv.) were then added in one portion. The mixture was stirred at $45{ }^{\circ} \mathrm{C}$ for 12 hours. Silica was added to the mixture and the solvent was removed under vacuum. The resulting solid was placed on a column of silica gel and purified by 
column chromatography $(\mathrm{PE} / \mathrm{EtOAc}=9: 1)$ to give 7. Yield: $34.3 \mathrm{mg}(72 \%)$; time: $12.0 \mathrm{~h}$; colorless liquid; TLC, $\mathrm{R}_{\mathrm{f}}=0.48(\mathrm{PE}: \mathrm{EtOAc}=8: 2) ;{ }^{1} \mathrm{H}$ NMR $\left(\mathrm{CDCl}_{3}, 400 \mathrm{MHz}\right): \delta 7.31(\mathrm{~d}, 2 \mathrm{H}, \mathrm{J}=8.4 \mathrm{~Hz}), 6.93(\mathrm{~d}, 2 \mathrm{H}, \mathrm{J}=8.4$ Hz), 3.82 (s, 3H), 2.63 (t, 2H, J = 7.6 Hz), 1.74-1.67 (m, 2H), 1.36-1.33 (m, 4H), 0.91 (t, 3H, J = 6.8 Hz); ${ }^{13} \mathrm{C}\left\{{ }^{1} \mathrm{H}\right\} \mathrm{NMR}\left(\mathrm{CDCl}_{3}, 100 \mathrm{MHz}\right): \delta 198.6,160.5,136.0,118.7,114.8,55.3,43.4,31.1,25.3,22.3,13.8$; HRMS (ESI) m/z calcd. for $\mathrm{C}_{13} \mathrm{H}_{18} \mathrm{O}_{2} \mathrm{SNa}[\mathrm{M}+\mathrm{Na}]^{+}: 261.0920$, found: 261.0928.

\section{Synthesis of 1-Benzyl-4-butyl-5-((4-methoxyphenyl)thio)-1H-1,2,3-triazole (8) ${ }^{6}$}

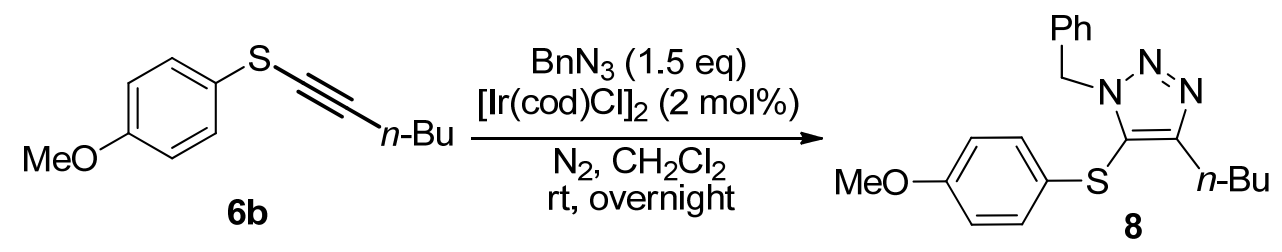

To an oven-dried 5-mL vial was added hex-1-yn-1-yl(4-methoxyphenyl)sulfane (44 mg, $0.20 \mathrm{mmol}$ ), benzyl azide (40 mg, $0.30 \mathrm{mmol}),[\operatorname{Ir}(\mathrm{COD}) \mathrm{Cl}]_{2}(2.7 \mathrm{mg}, 4.0 \mu \mathrm{mol})$, and $\mathrm{DCM}(2.0 \mathrm{~mL})$ under nitrogen. The reaction mixture was stirred at room temperature overnight, and then concentrated under reduced pressure. The residue was purified by column chromatography $(\mathrm{PE} / \mathrm{EtOAc}=5: 1)$ to give 8. Yield: $54 \mathrm{mg}(77 \%)$; time: $2.5 \mathrm{~h}$; yellow liquid; TLC, $\mathrm{R}_{\mathrm{f}}=0.26(\mathrm{PE} / \mathrm{EtOAc}=5: 1) ;{ }^{1} \mathrm{H} \mathrm{NMR}\left(\mathrm{CDCl}_{3}, 400 \mathrm{MHz}\right): \delta 7.16-7.10(\mathrm{~m}, 5 \mathrm{H}), 6.78(\mathrm{~d}, 2 \mathrm{H}, \mathrm{J}=$ $8.8 \mathrm{~Hz}), 6.61(\mathrm{~d}, 2 \mathrm{H}, \mathrm{J}=8.8 \mathrm{~Hz}), 5.43(\mathrm{~s}, 2 \mathrm{H}), 3.66(\mathrm{~s}, 3 \mathrm{H}), 2.61(\mathrm{t}, 2 \mathrm{H}, \mathrm{J}=7.6 \mathrm{~Hz}), 1.58-1.50(\mathrm{~m}, 2 \mathrm{H}), 1.30-$ $1.20(\mathrm{~m}, 2 \mathrm{H}), 0.80(\mathrm{t}, 3 \mathrm{H}, \mathrm{J}=7.2 \mathrm{~Hz}) ;{ }^{13} \mathrm{C}\left\{{ }^{1} \mathrm{H}\right\} \mathrm{NMR}\left(\mathrm{CDCl}_{3}, 100 \mathrm{MHz}\right): \delta 159.0,152.8,134.9,130.0,128.6$, $128.0,127.8,124.9,123.9,114.9,55.3,52.1,31.1,25.1,22.4,13.7$. 
Table S1. Condition Optimization for $\alpha$-Alkynylthiolation of $\beta$-Keto Esters ${ }^{a}$
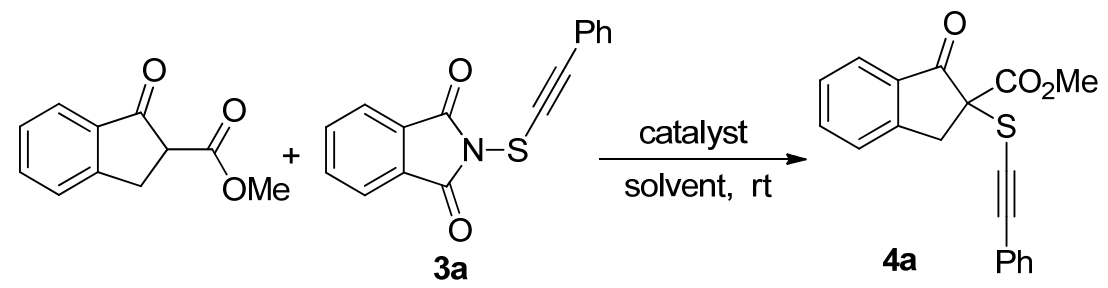

\begin{tabular}{lllcc}
\hline Entry & Catalyst & Solvent & Temp. & Yield $^{b}(\%)$ \\
\hline 1 & DABCO (2.0 eq) & $\mathrm{CH}_{2} \mathrm{Cl}_{2}$ & $\mathrm{rt}$ & 93 \\
2 & $\mathrm{DABCO}(1.0 \mathrm{eq})$ & $\mathrm{CH}_{2} \mathrm{Cl}_{2}$ & $\mathrm{rt}$ & 87 \\
3 & $\mathrm{DABCO}(0.5 \mathrm{eq})$ & $\mathrm{CH}_{2} \mathrm{Cl}_{2}$ & $\mathrm{rt}$ & 88 \\
4 & $\mathrm{DABCO}(0.3 \mathrm{eq})$ & $\mathrm{CH}_{2} \mathrm{Cl}_{2}$ & $\mathrm{rt}$ & 91 \\
5 & $\mathrm{DABCO}(0.2 \mathrm{eq})$ & $\mathrm{CH}_{2} \mathrm{Cl}_{2}$ & $\mathrm{rt}$ & 76 \\
6 & $\mathrm{DABCO}(0.2 \mathrm{eq})$ & $\mathrm{CH}_{2} \mathrm{Cl}_{2}$ & reflux & 95 \\
7 & $\mathrm{DABCO}(0.2 \mathrm{eq})$ & $\mathrm{THF}^{2}$ & $45^{\circ} \mathrm{C}$ & 53 \\
8 & $\mathrm{DABCO}(0.2 \mathrm{eq})$ & $\mathrm{EtOAc}^{\circ}$ & $45^{\circ} \mathrm{C}$ & 70 \\
9 & $\mathrm{Et} \mathrm{N}_{3}(0.2 \mathrm{eq})$ & $\mathrm{CH}_{2} \mathrm{Cl}_{2}$ & reflux & 82 \\
10 & $\mathrm{Cs} \mathrm{CO}_{3}(0.2 \mathrm{eq})$ & $\mathrm{CH}_{2} \mathrm{Cl}_{2}$ & reflux & 93 \\
11 & $\mathrm{Na}_{2} \mathrm{CO}_{3}(0.2 \mathrm{eq})$ & $\mathrm{CH}_{2} \mathrm{Cl}_{2}$ & reflux & 90 \\
12 & $\mathrm{~K}_{2} \mathrm{CO}_{3}(0.2 \mathrm{eq})$ & $\mathrm{CH}_{2} \mathrm{Cl}_{2}$ & reflux & 90 \\
13 & $\mathrm{DBU}(0.2$ eq $)$ & $\mathrm{CH}_{2} \mathrm{Cl}_{2}$ & reflux & 78 \\
\hline
\end{tabular}

${ }^{a}$ Reaction conditions: Methyl 1-indanone-2-carboxylate $(0.1 \mathrm{mmol}), 3 a(0.15 \mathrm{mmol})$, catalyst, in the solvent $(1 \mathrm{~mL})$ stirred at indicated temperature for $2-3 \mathrm{~h}$.

${ }^{b}$ Isolated yield. 
Table S2. Condition Optimization for Cu-Catalyzed Alkynylthiolation of Boronic acids ${ }^{a}$

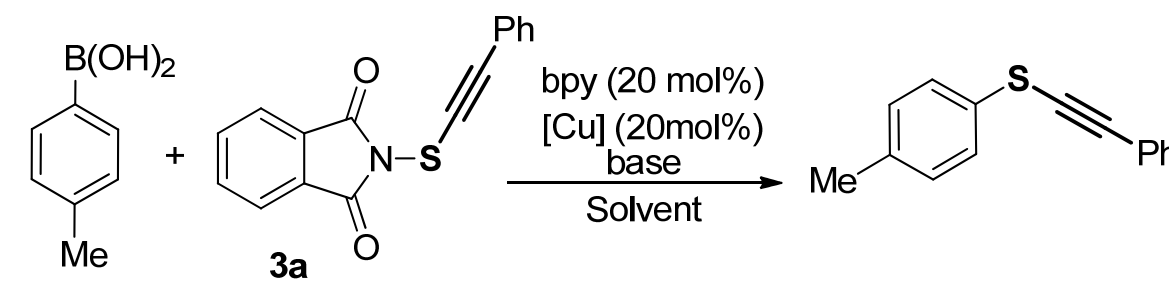

\begin{tabular}{lllllc}
\hline Entry & Catalyst & Base & Solvent & Temp. $\left({ }^{\circ} \mathrm{C}\right)$ & Yield $^{\mathrm{b}}(\%)$ \\
\hline 1 & $\mathrm{CuCl}$ & $\mathrm{K}_{2} \mathrm{CO}_{3}$ & $\mathrm{DME}$ & 0 & 31 \\
2 & $\mathrm{CuCl}$ & $\mathrm{K}_{2} \mathrm{CO}_{3}$ & $\mathrm{DME}$ & 45 & 57 \\
3 & $\mathrm{CuCl}$ & $\mathrm{K}_{2} \mathrm{CO}_{3}$ & $\mathrm{DME}$ & 60 & 62 \\
4 & $\mathrm{CuCl}$ & $\mathrm{K}_{2} \mathrm{CO}_{3}$ & $\mathrm{DME}$ & 80 & 49 \\
5 & $\mathrm{Cul}$ & $\mathrm{K}_{2} \mathrm{CO}_{3}$ & $\mathrm{DME}$ & 60 & 48 \\
6 & $\mathrm{CuSal}$ & $\mathrm{K}_{2} \mathrm{CO}_{3}$ & $\mathrm{DME}$ & 60 & 43 \\
7 & $\mathrm{CuCl} \cdot 2 \mathrm{H}_{2} \mathrm{O}$ & $\mathrm{K}_{2} \mathrm{CO}_{3}$ & $\mathrm{DME}$ & 60 & 65 \\
8 & $\mathrm{CuSO}$ & $\mathrm{K}_{2} \mathrm{CO}_{3}$ & $\mathrm{DME}$ & 60 & 12 \\
9 & $\mathrm{Cu}(\mathrm{OAc})_{2}$ & $\mathrm{~K}_{2} \mathrm{CO}_{3}$ & $\mathrm{DME}$ & 60 & 48 \\
10 & $\mathrm{Cu}(\mathrm{OTf})_{2}$ & $\mathrm{~K}_{2} \mathrm{CO}_{3}$ & $\mathrm{DME}$ & 60 & 70 \\
$\mathbf{1 1}$ & $\mathrm{Cu}(\mathrm{OTf})_{2}$ & $\mathrm{~K}_{2} \mathrm{CO}_{3}$ & THF & 60 & 96 \\
12 & $\mathrm{Cu}(\mathrm{OTf})_{2}$ & $\mathrm{~K}_{2} \mathrm{CO}_{3}$ & $\mathrm{MeOH}$ & 60 & 30 \\
13 & $\mathrm{Cu}(\mathrm{OTf})_{2}$ & $\mathrm{~K}_{2} \mathrm{CO}_{3}$ & EtOAc & 60 & 89 \\
14 & $\mathrm{Cu}(\mathrm{OTf})_{2}$ & $\mathrm{DABCO}_{1}$ & THF & 60 & 37 \\
15 & $\mathrm{Cu}(\mathrm{OTf})_{2}$ & $\mathrm{Cs}_{2} \mathrm{CO}_{3}$ & THF & 60 & 45 \\
\hline
\end{tabular}

${ }^{a}$ Reaction conditions: $p$-tolylboronic acid $(0.1 \mathrm{mmol}), 3 a(0.15 \mathrm{mmol})$, catalyst $(20 \mathrm{~mol} \%)$, bpy $(20 \mathrm{~mol} \%)$, and base $(0.2 \mathrm{mmol})$ in the solvent $(1 \mathrm{~mL})$ stirred at indicated temperature for 2-3 $\mathrm{h}$.

${ }^{b}$ Isolated yield. 
Figure S1. Crystal Structure of 3a (CCDC 1916070; ellipsoids are set at 50\%) and the Corresponding

Data

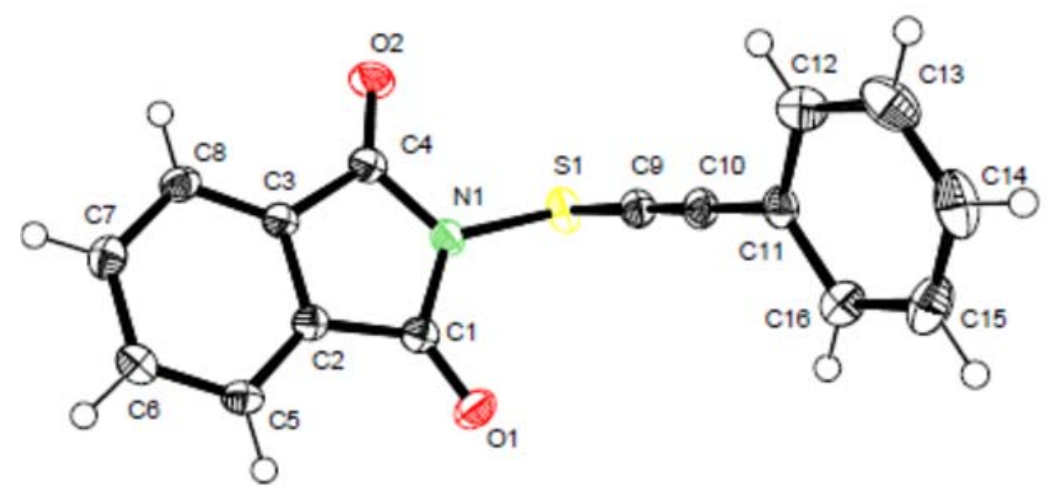

\begin{tabular}{|c|c|}
\hline \multicolumn{2}{|l|}{ Crystal data } \\
\hline Chemical formula & $\underline{\mathrm{C}}_{16} \mathrm{H}_{9} \mathrm{NO}_{2} \mathrm{~S}$ \\
\hline$M_{\mathrm{r}}$ & $\underline{279.30}$ \\
\hline Crystal system, space group & Triclinic, $\underline{P}$ \\
\hline Temperature $(\mathrm{K})$ & 150 \\
\hline$a, b, c(\AA)$ & $7.0563(4), 7.9330(4), \underline{11.8556(7)}$ \\
\hline$\alpha, \beta, \gamma\left(^{\circ}\right)$ & $\underline{87.855(5)}, \underline{89.294(5)}, \underline{73.584(5)}$ \\
\hline$V\left(\AA^{3}\right)$ & $\underline{636.15(6)}$ \\
\hline$Z$ & $\underline{2}$ \\
\hline Radiation type & $\underline{\text { Mo } K \alpha}$ \\
\hline$\mu\left(\mathrm{mm}^{-1}\right)$ & 0.25 \\
\hline Crystal size (mm) & $\underline{0.47} \times \underline{0.27} \times \underline{0.10}$ \\
\hline \multicolumn{2}{|l|}{ Data collection } \\
\hline Diffractometer & $\underline{\text { SuperNova, Dual, } \mathrm{Cu} \text { at zero, Atlas diffractometer }}$ \\
\hline Absorption correction & $\begin{array}{l}\frac{\text { Gaussian }}{\text { CrysAlis } P R O ~ 1.171 .38 .43 f \text { (Rigaku Oxford Diffraction, 2015) Numerical }} \\
\text { absorption correction based on gaussian integration over a multifaceted } \\
\text { crystal model Empirical absorption correction using spherical harmonics, } \\
\text { implemented in SCALE3 ABSPACK scaling algorithm. }\end{array}$ \\
\hline$T_{\min }, T_{\max }$ & $\underline{0.490}, \underline{1.000}$ \\
\hline
\end{tabular}




\begin{tabular}{|c|c|}
\hline $\begin{array}{l}\text { No. of measured, independent } \\
\text { and observed }[\underline{I>2 \sigma(I)}] \\
\text { reflections }\end{array}$ & $\underline{11032}, \underline{3180}, \underline{2687}$ \\
\hline$R_{\text {int }}$ & $\underline{0.029}$ \\
\hline$(\sin \theta / \lambda)_{\max }\left(\AA^{-1}\right)$ & 0.698 \\
\hline \multicolumn{2}{|l|}{ Refinement } \\
\hline$R\left[F^{2}>2 \sigma\left(F^{2}\right)\right], w R\left(F^{2}\right), S$ & $\underline{0.038}, \underline{0.091}, \underline{1.04}$ \\
\hline No. of reflections & $\underline{3180}$ \\
\hline No. of parameters & 181 \\
\hline H-atom treatment & $\underline{\text { H-atom parameters constrained }}$ \\
\hline$\Delta \rho_{\max }, \Delta \rho_{\min }\left(\mathrm{e} \AA^{-3}\right)$ & $\underline{0.28}, \underline{-0.34}$ \\
\hline
\end{tabular}

Computer programs: CrysAlis PRO 1.171.38.43f (Rigaku OD, 2015), SHELXS2013 (Sheldrick, 2013), SHELXL2013 (Sheldrick, 2013).

\section{References}

[1] Graf, T. A.; Yoo, J.; Brummett, A. B.; Lin, R.; Wohlgenannt, M.; Quinn, D.; Bowden, N. B. Macromolecules 2012, 45, 8193.

[2] (a) Zhu, D.; Gu, Y.; Lu, L.; Shen, Q. J. Am. Chem. Soc. 2015, 137, 10547. (b) Hutchinson, S. A.; Baker, S. P.; Linden, J.; Scammells, P. J. Bioorg. Med. Chem. 2004, 12, 4877.

[3] (a) Wang, T.; Chen, S.; Shao, A.; Gao, M.; Huang, Y.; Lei, A. Org. Lett. 2015, 17, 118. (b) Jiang, Z.-G.;

Shi, K.; Lin, Y.-M.; Wang, Q.-M. Chem. Commun. 2014, 50, 2353.

[4] Yang, Y.; Dong, W.; Guo, Y.; Rioux, R. M. Green Chem. 2013, 15, 3170.

[5] Waldecker, B.; Kraft, F.; Golz, C.; Alcarazo, M. Angew. Chem. Int. Ed. 2018, 57, 12538.

[6] Ding, S.; Jia, G.; Sun, J. Angew. Chem. Int. Ed. 2014, 53, 1877.

[7] Peńa, J.; Talavera, G.; Waldecker, B.; Alcarazo, M. Chem. Eur. J. 2017, 23, 75. 


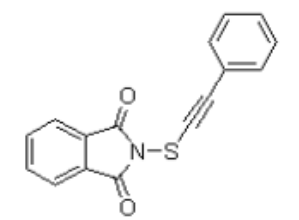

${ }^{1} \mathrm{HNMR} 500 \mathrm{MHz} \mathrm{CDCl}_{3}$

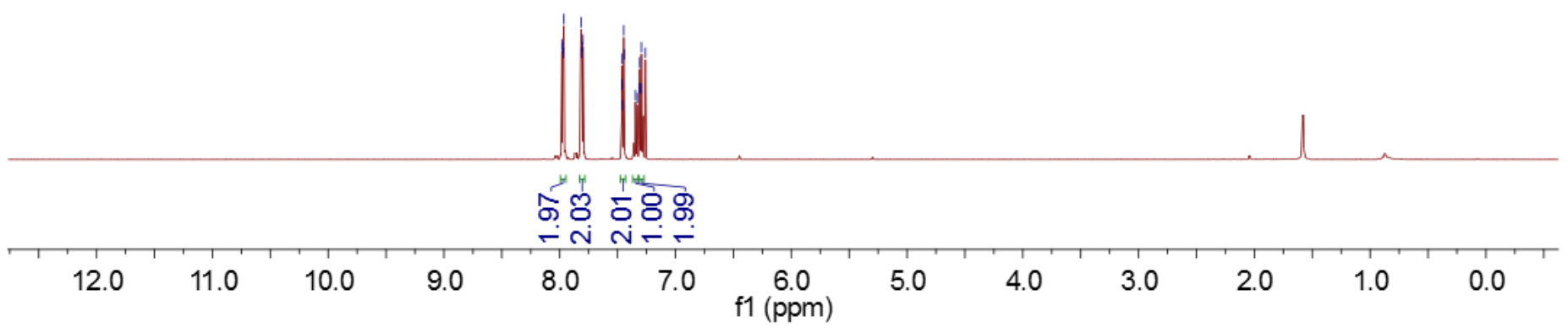




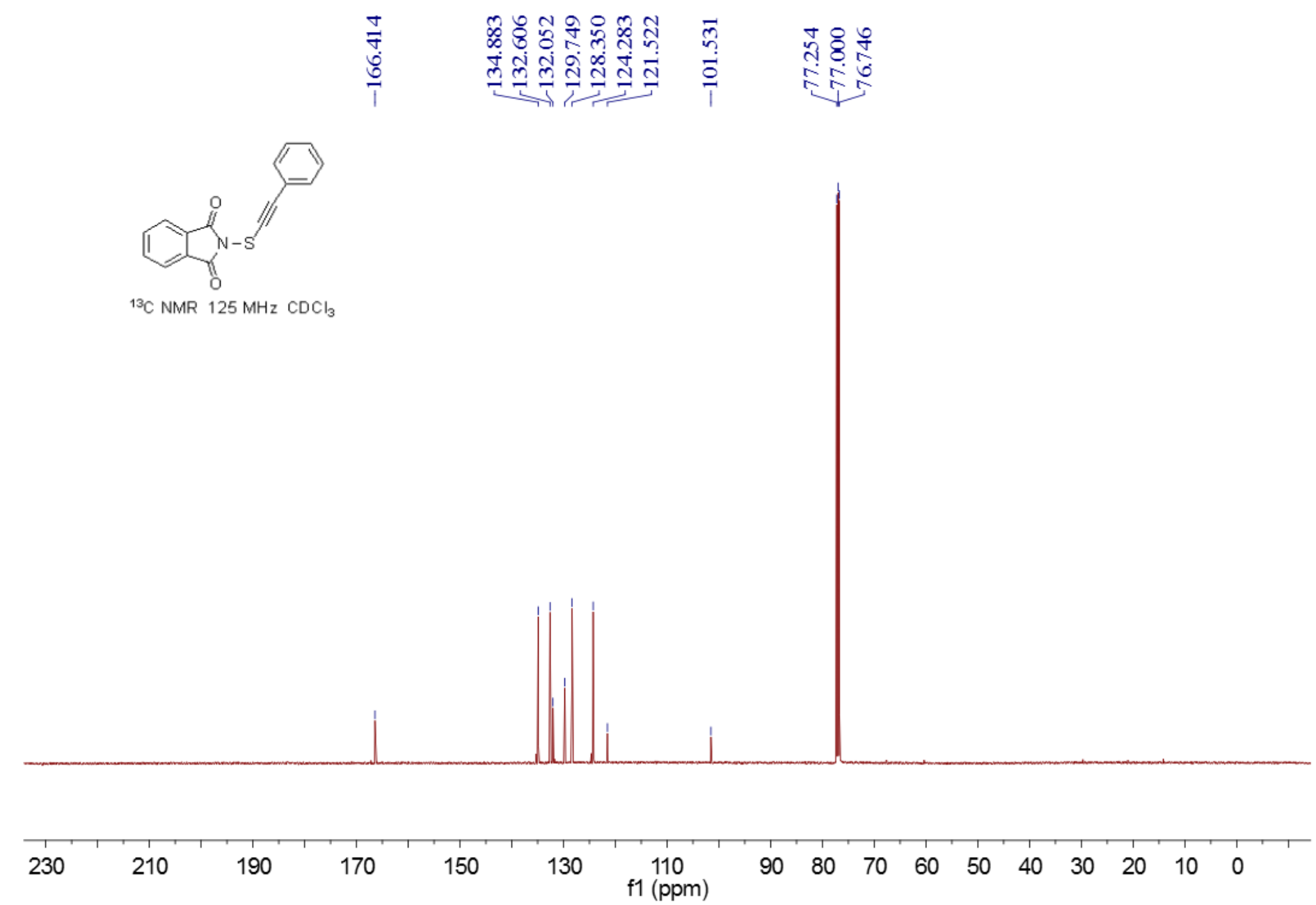




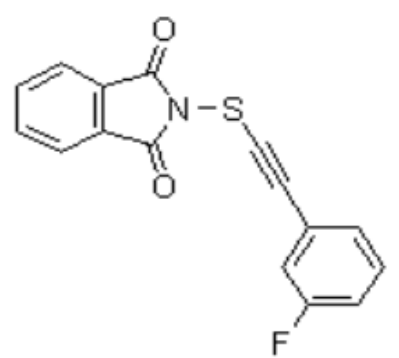

${ }^{1} \mathrm{H} \mathrm{NMR} 400 \mathrm{MHz} \mathrm{CDCl}_{3}$

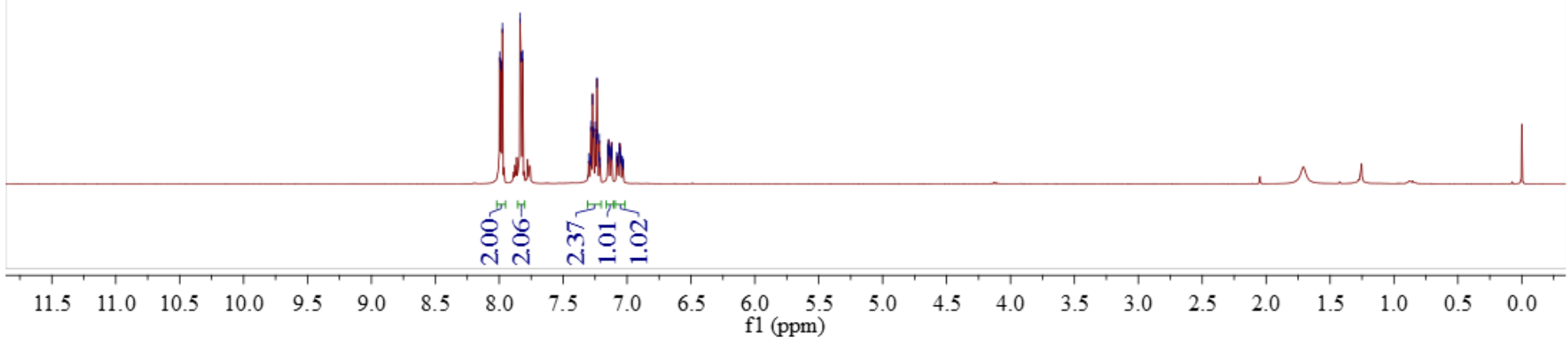




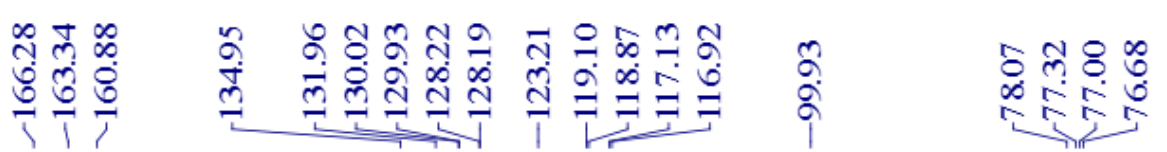

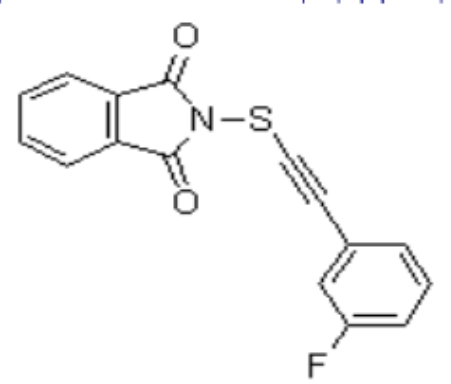

${ }^{13} \mathrm{C} \mathrm{NMR} 100 \mathrm{MHz} \mathrm{CDCl}_{3}$

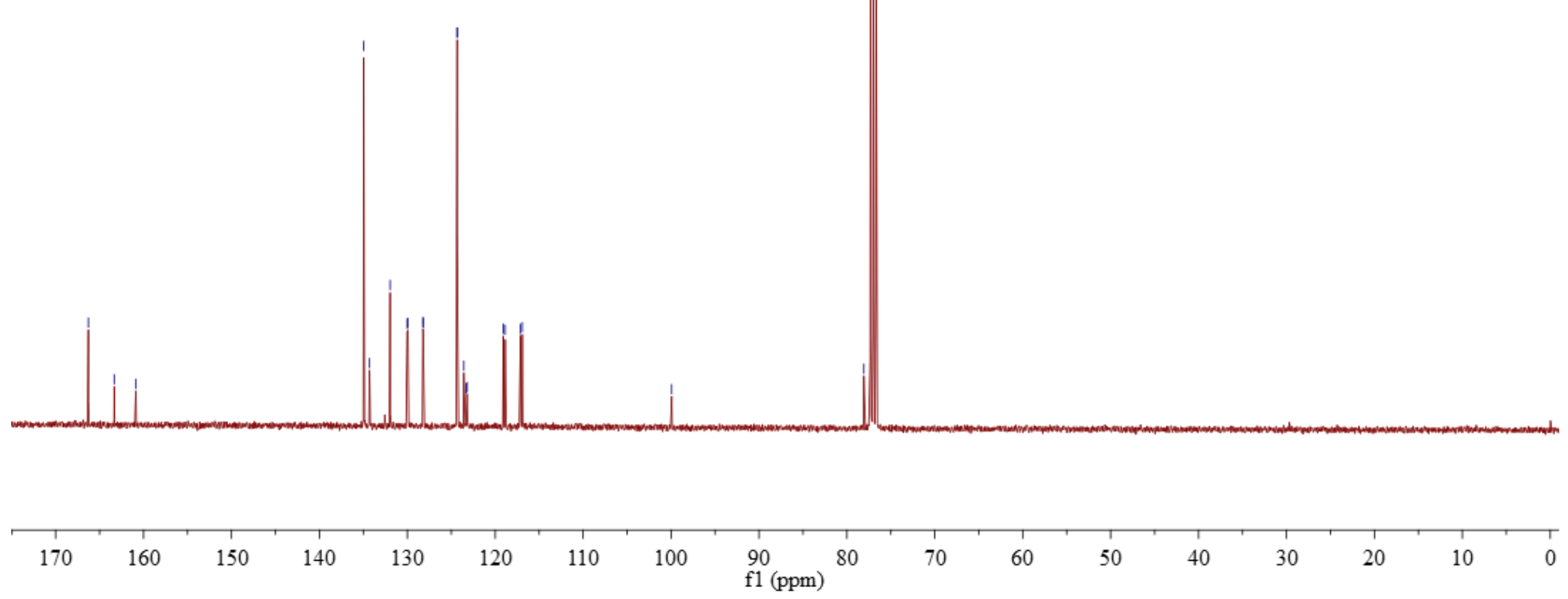


कล

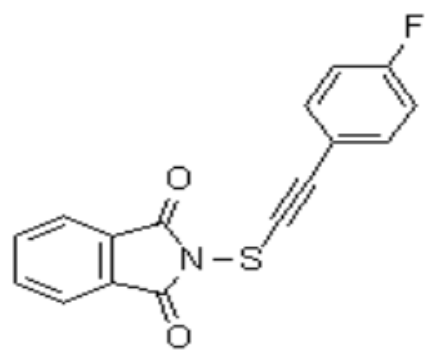

${ }^{1} \mathrm{H} N \mathrm{MR} 400 \mathrm{MHz} \mathrm{CDCl}_{3}$

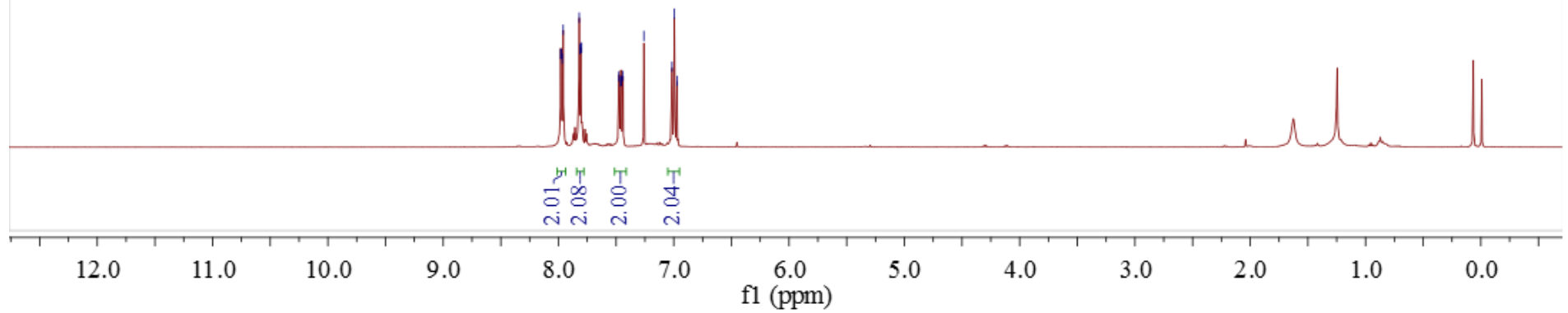



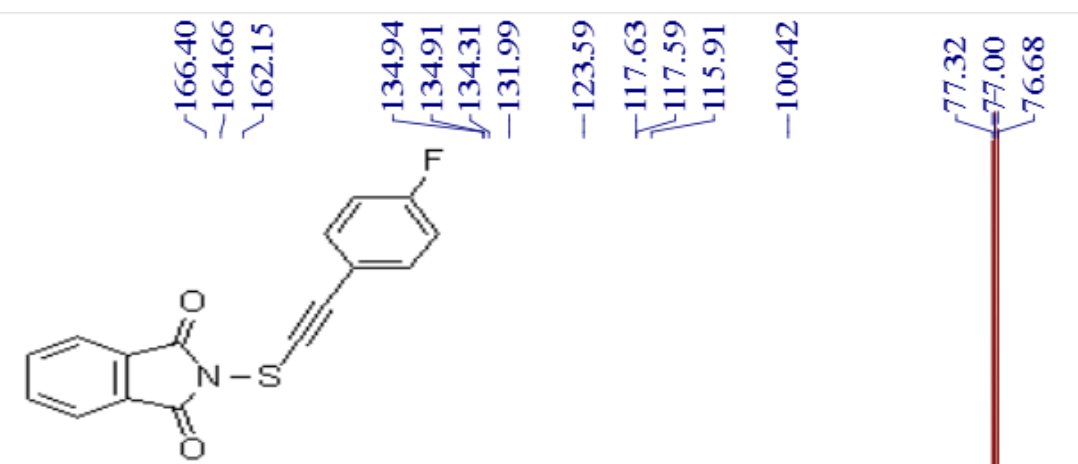

${ }^{13} \mathrm{C} \mathrm{NMR} 100 \mathrm{MHz} \mathrm{CDCl}_{3}$

$\begin{array}{lllllllllll}200 & 190 & 180 & 170 & 160 & 150 & 140 & 130 & 120 & 110 & \begin{array}{l}100 \\ \mathrm{f} 1(\mathrm{ppm})\end{array}\end{array}$ 


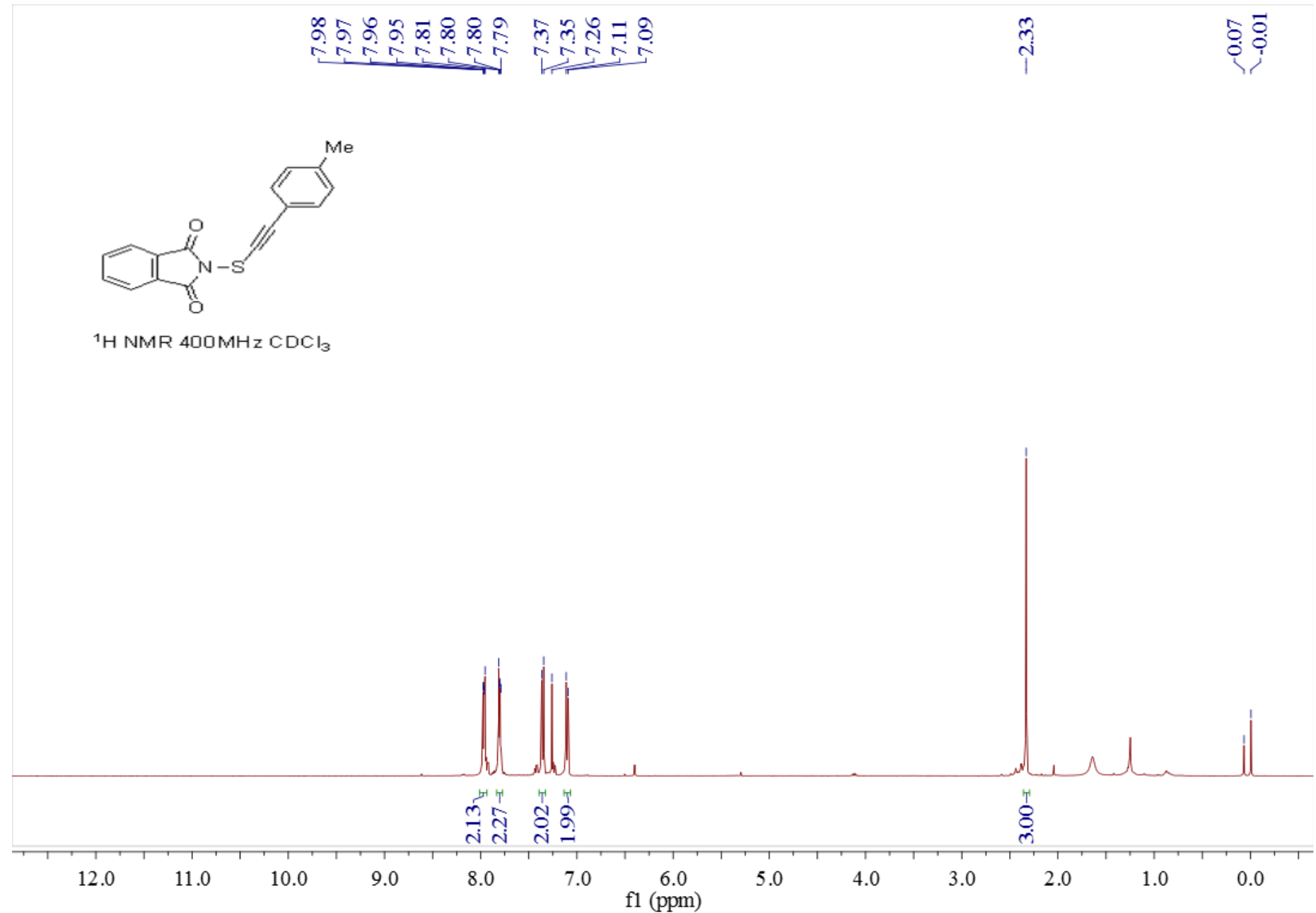




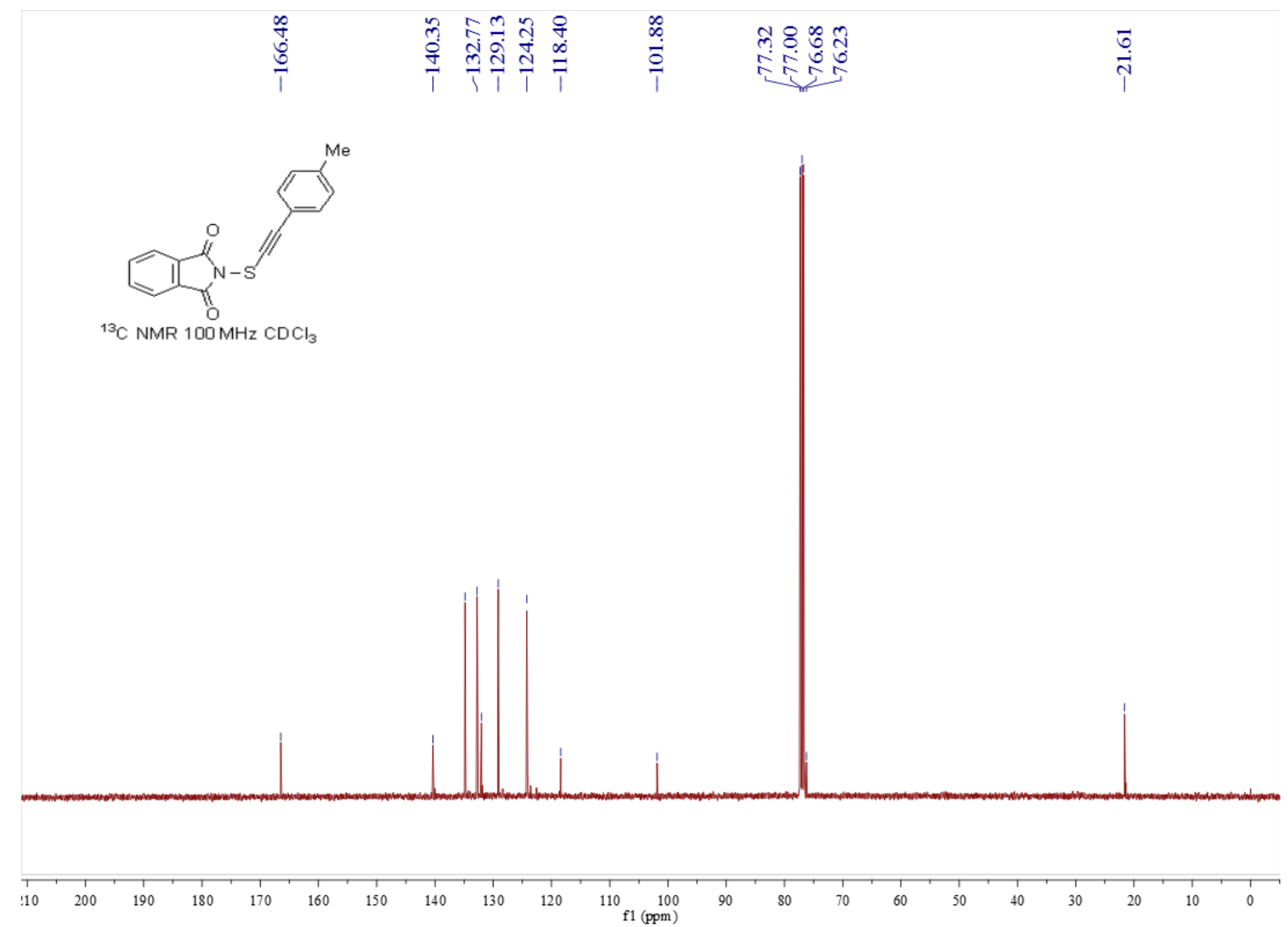


舟

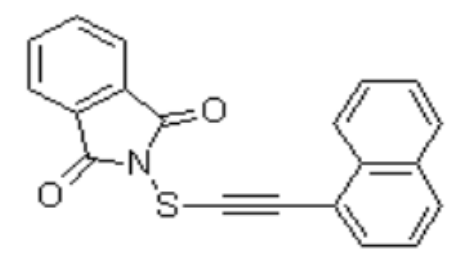

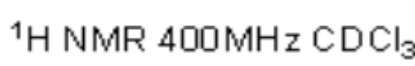

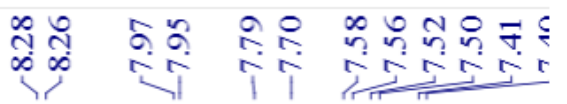
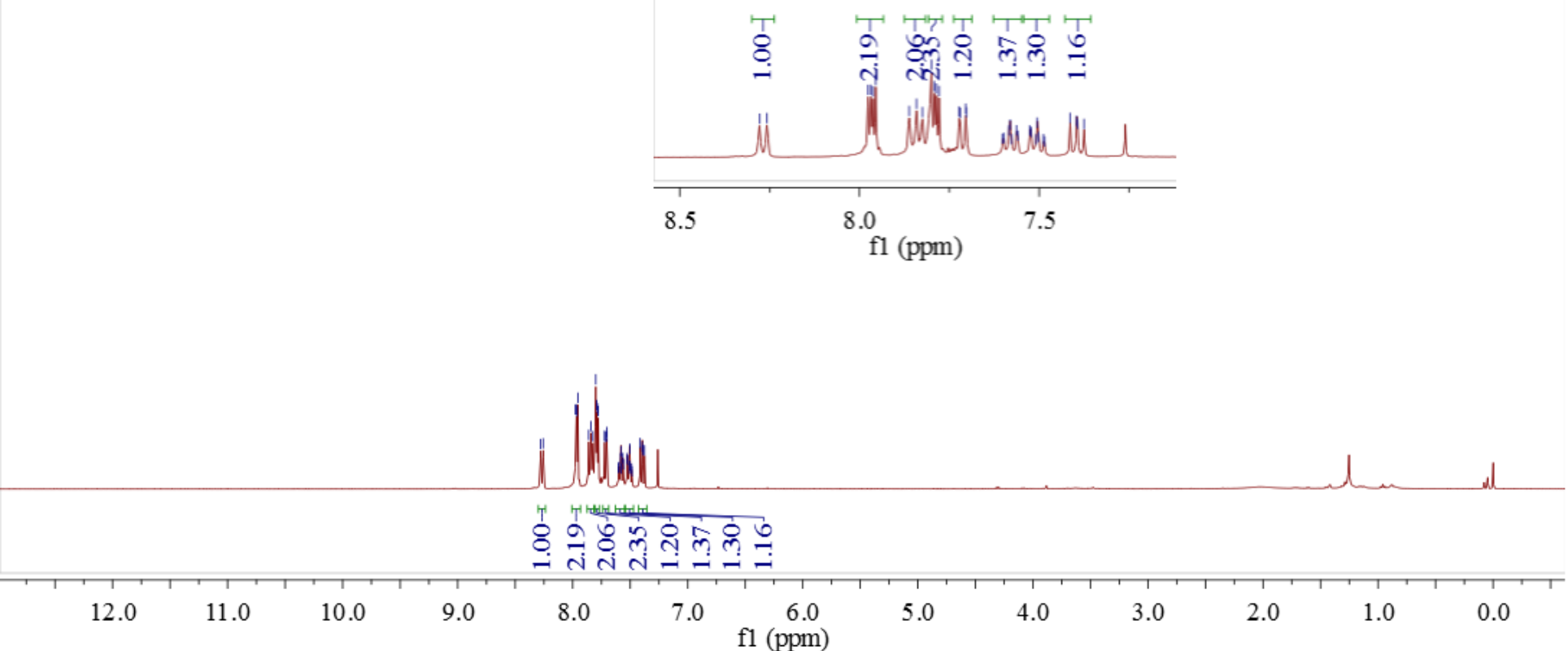


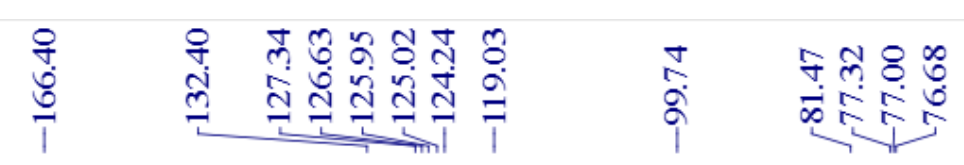

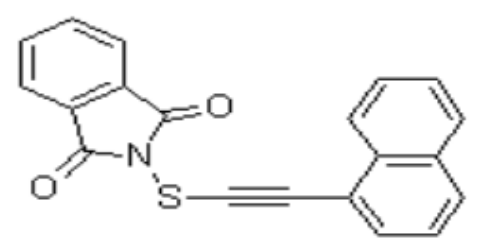

${ }^{13} \mathrm{C} \mathrm{NMR} 100 \mathrm{MHz} \mathrm{CDCl}_{3}$

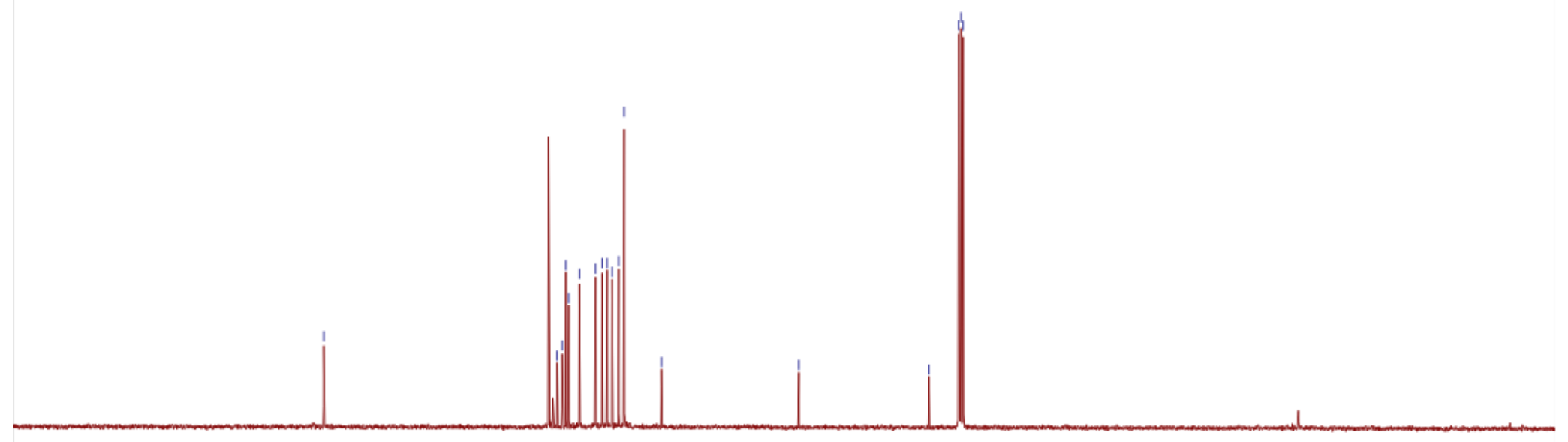

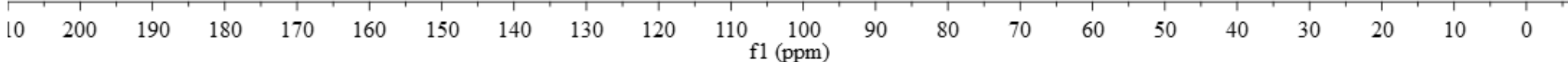




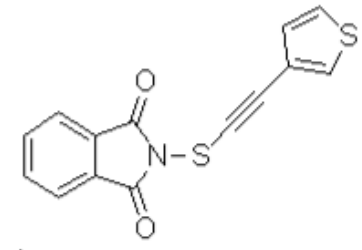

${ }^{1} \mathrm{HNMR} 400 \mathrm{MHz} \quad \mathrm{CDCl}_{3}$

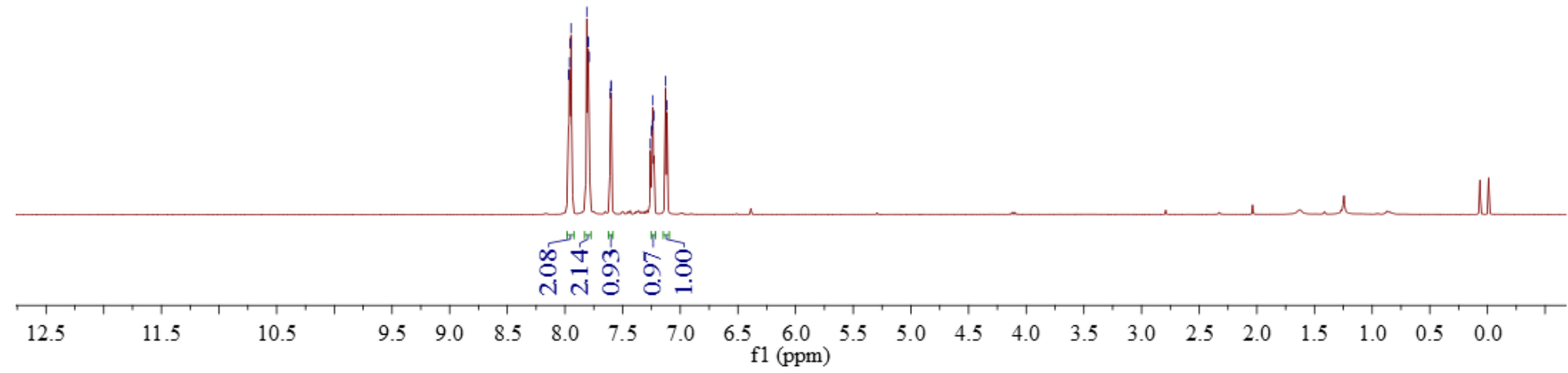



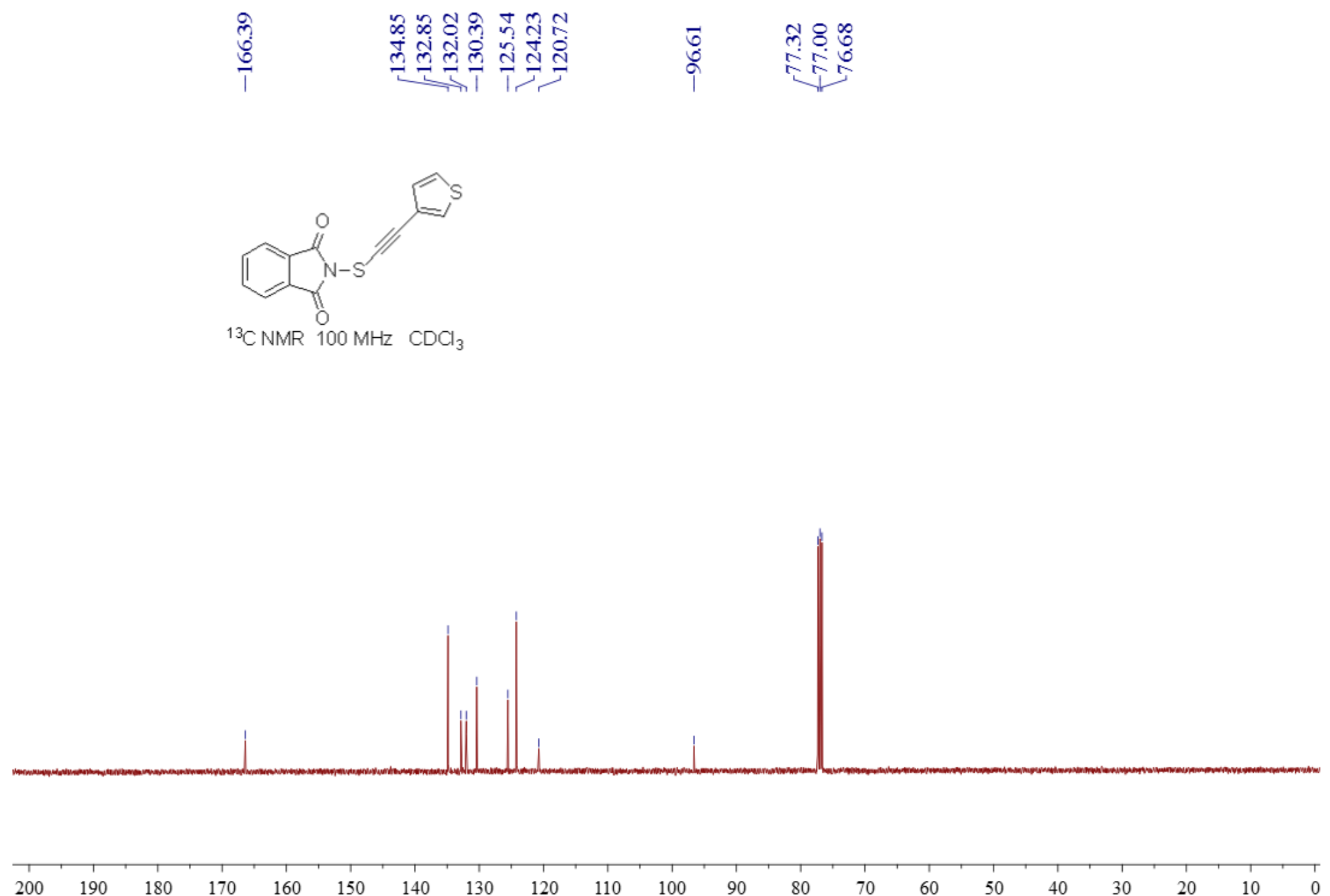

$\mathrm{f} 1(\mathrm{ppm})$ 


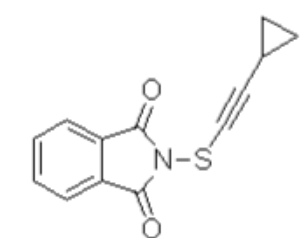

${ }^{1} \mathrm{HNMR} 400 \mathrm{MHz} \quad \mathrm{CDCl}_{3}$

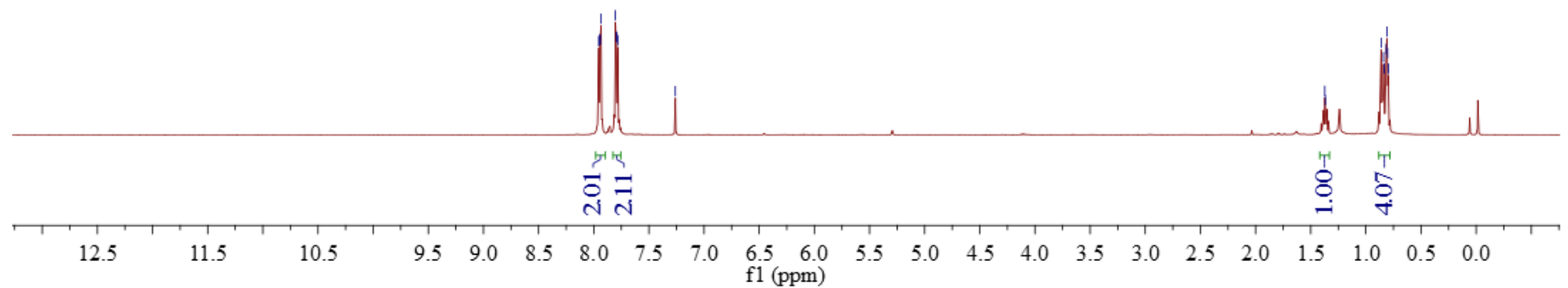



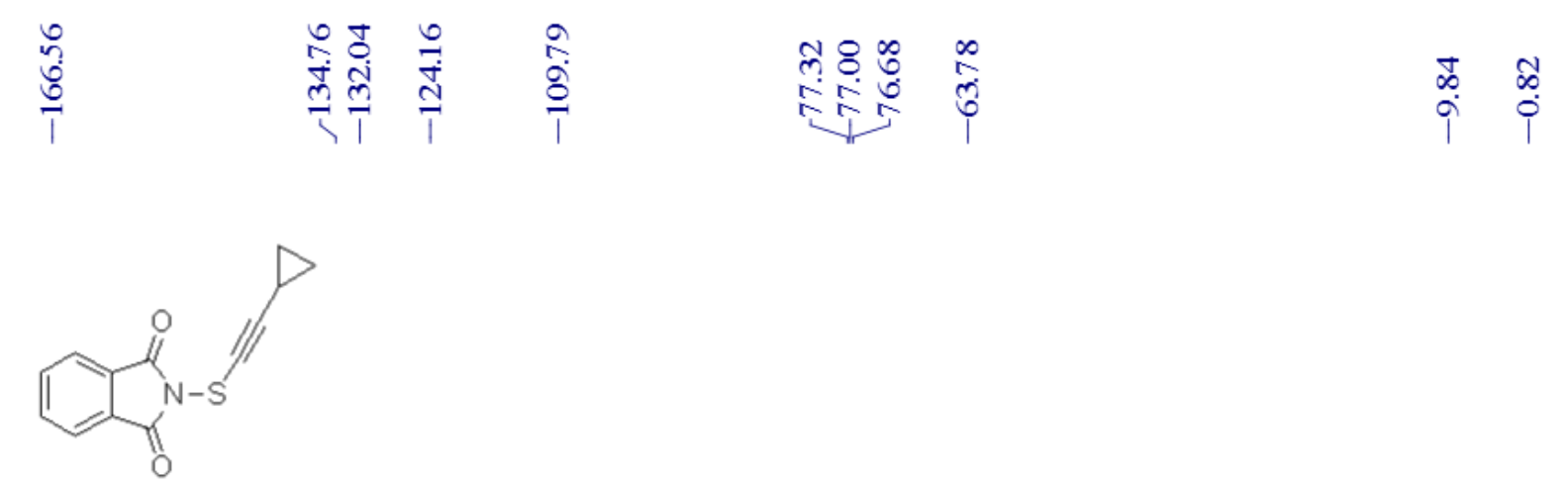

${ }^{13} \mathrm{CNMR} \quad 100 \mathrm{MHz} \quad \mathrm{CDCl}_{3}$

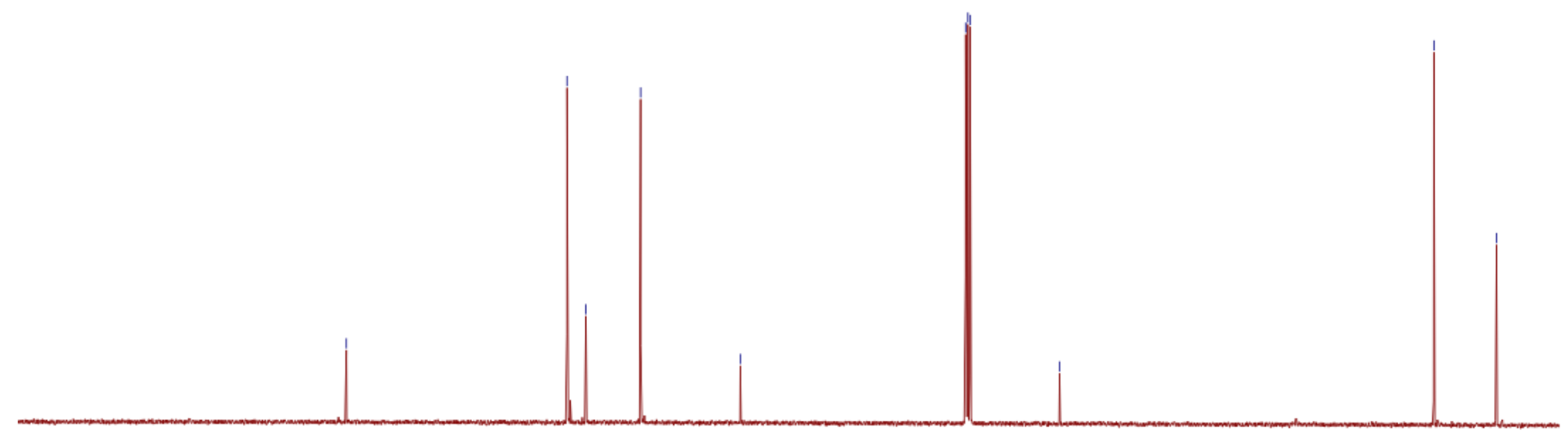

$\begin{array}{llllllllllll}210 & 200 & 190 & 180 & 170 & 160 & 150 & 140 & 130 & 120 & 110 & 100\end{array}$

f1 (ppm) 


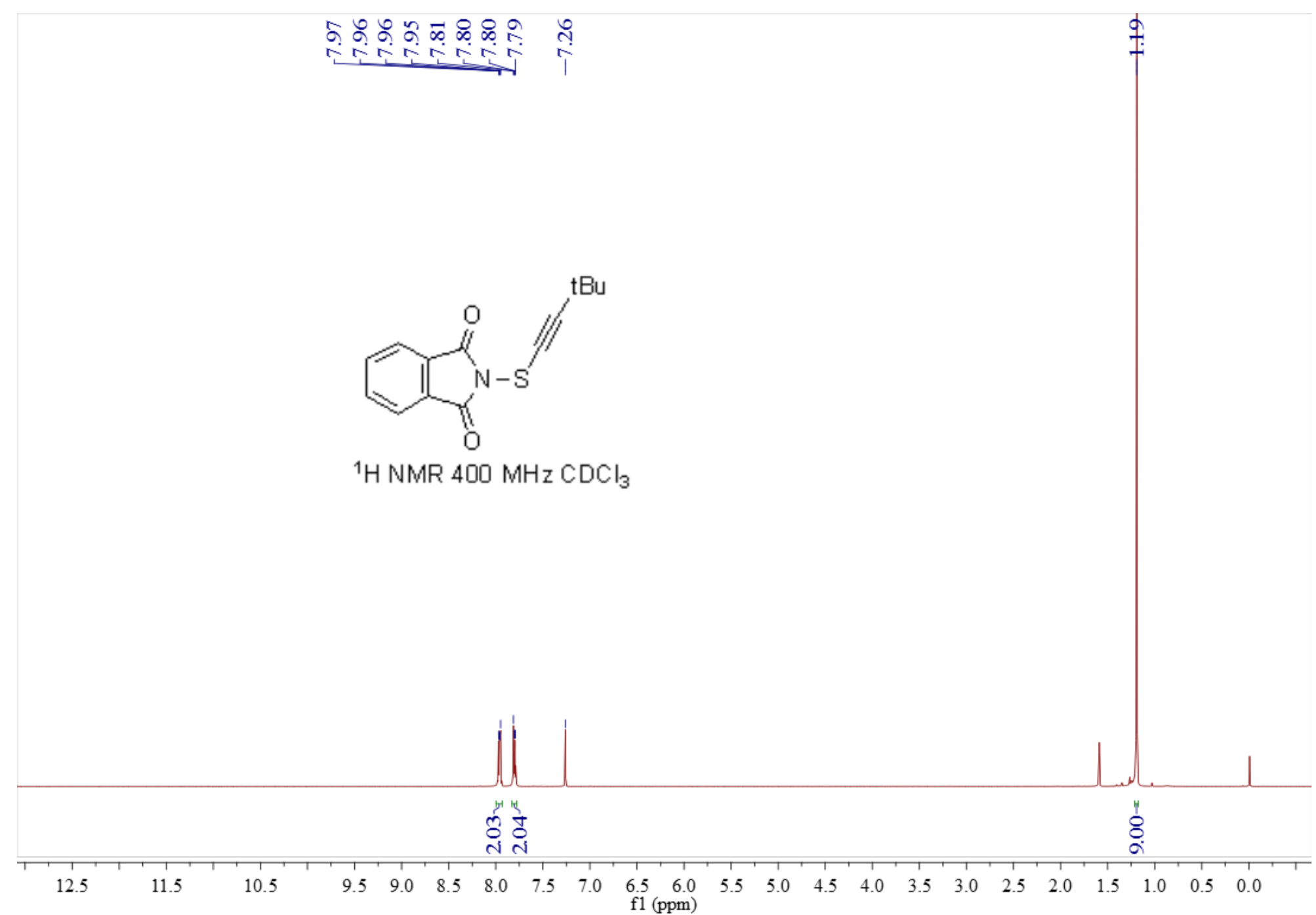




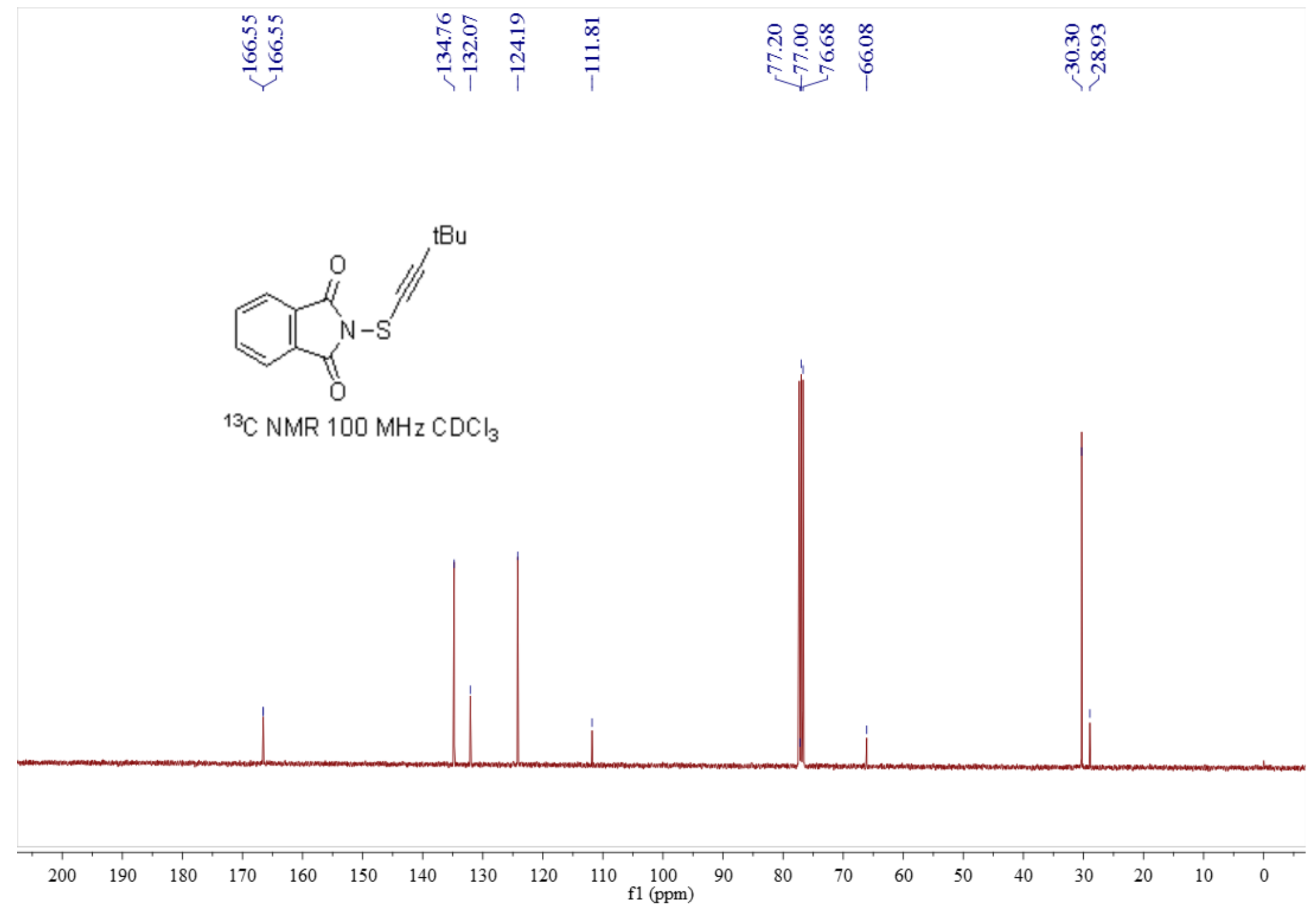




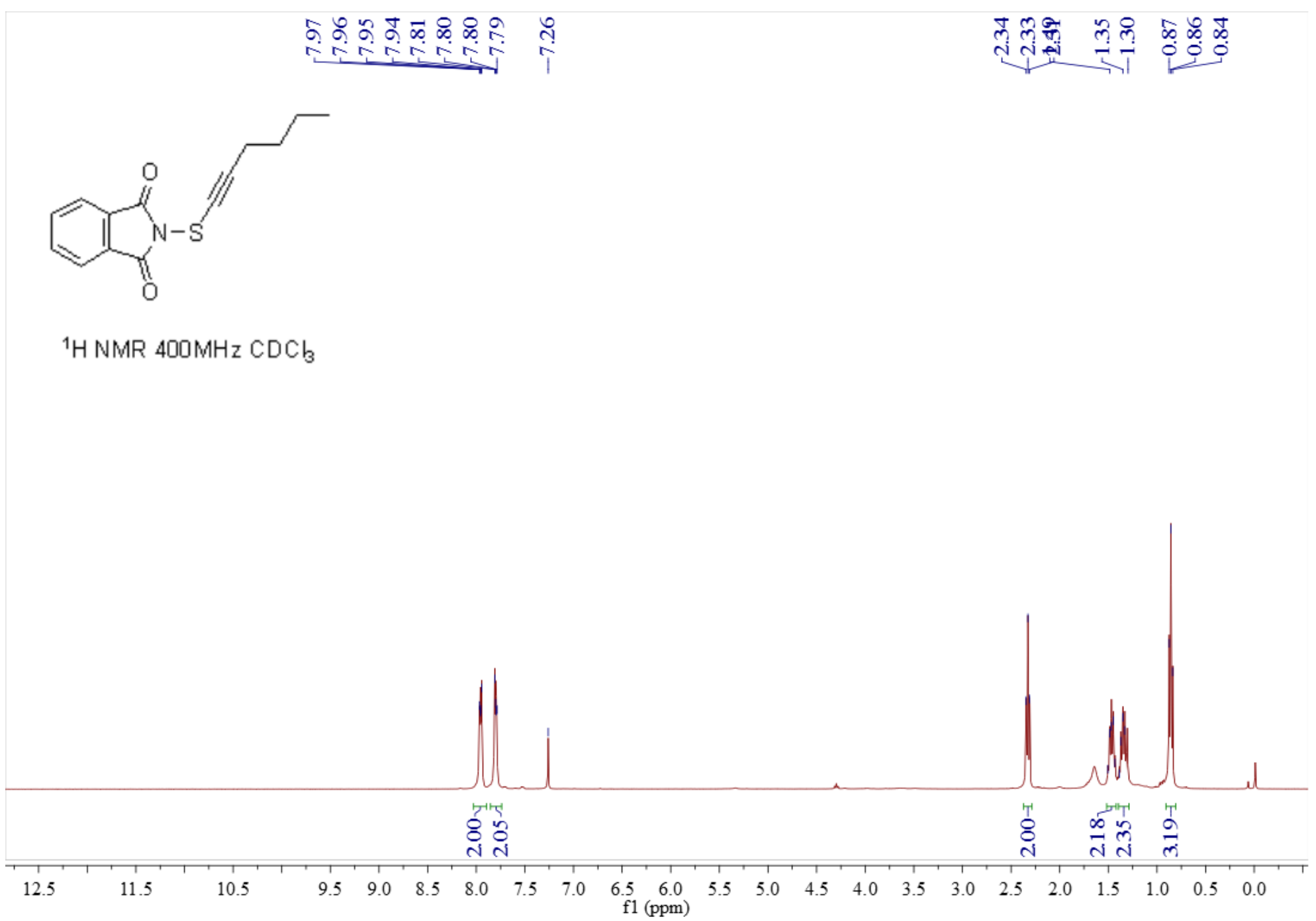




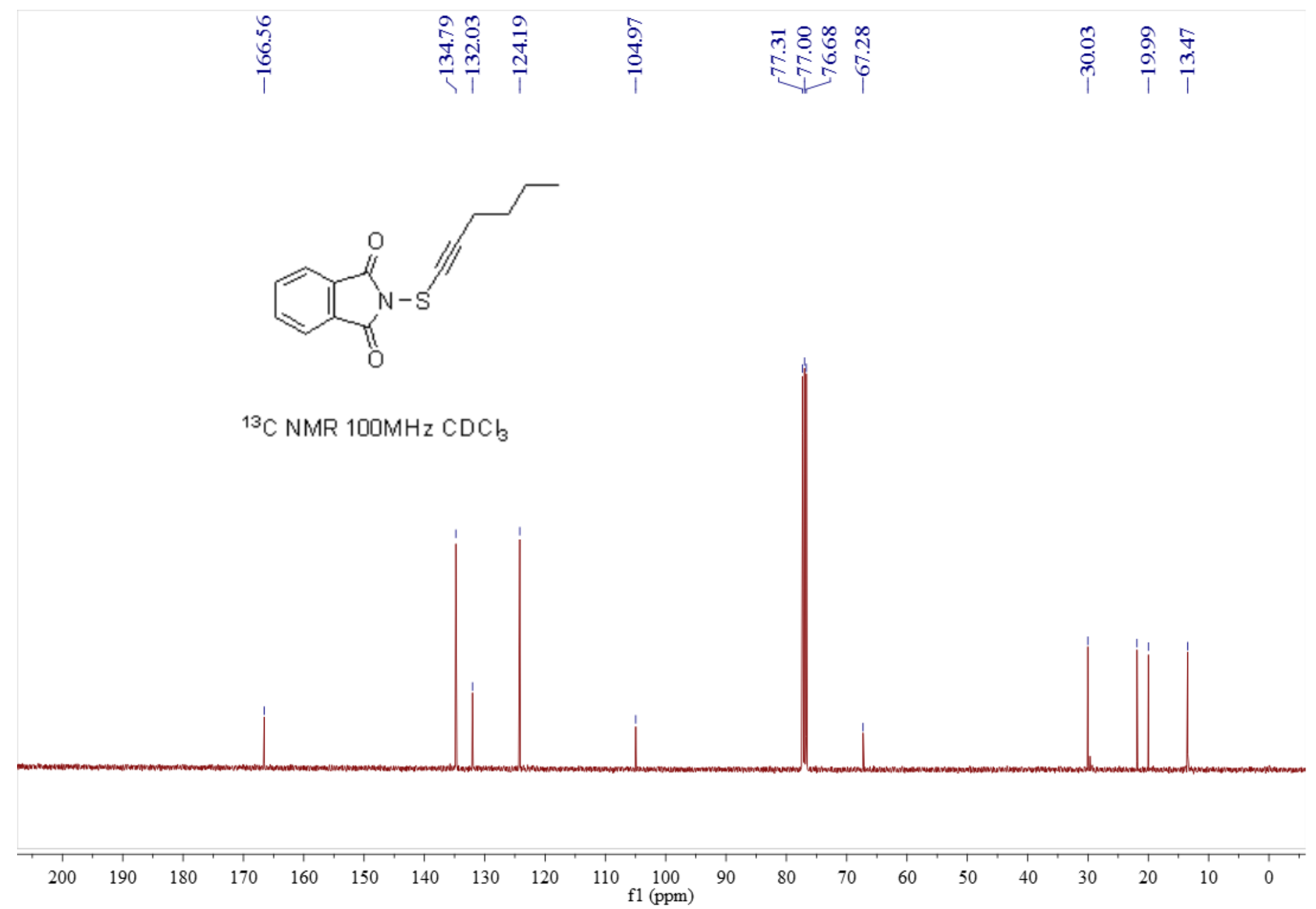




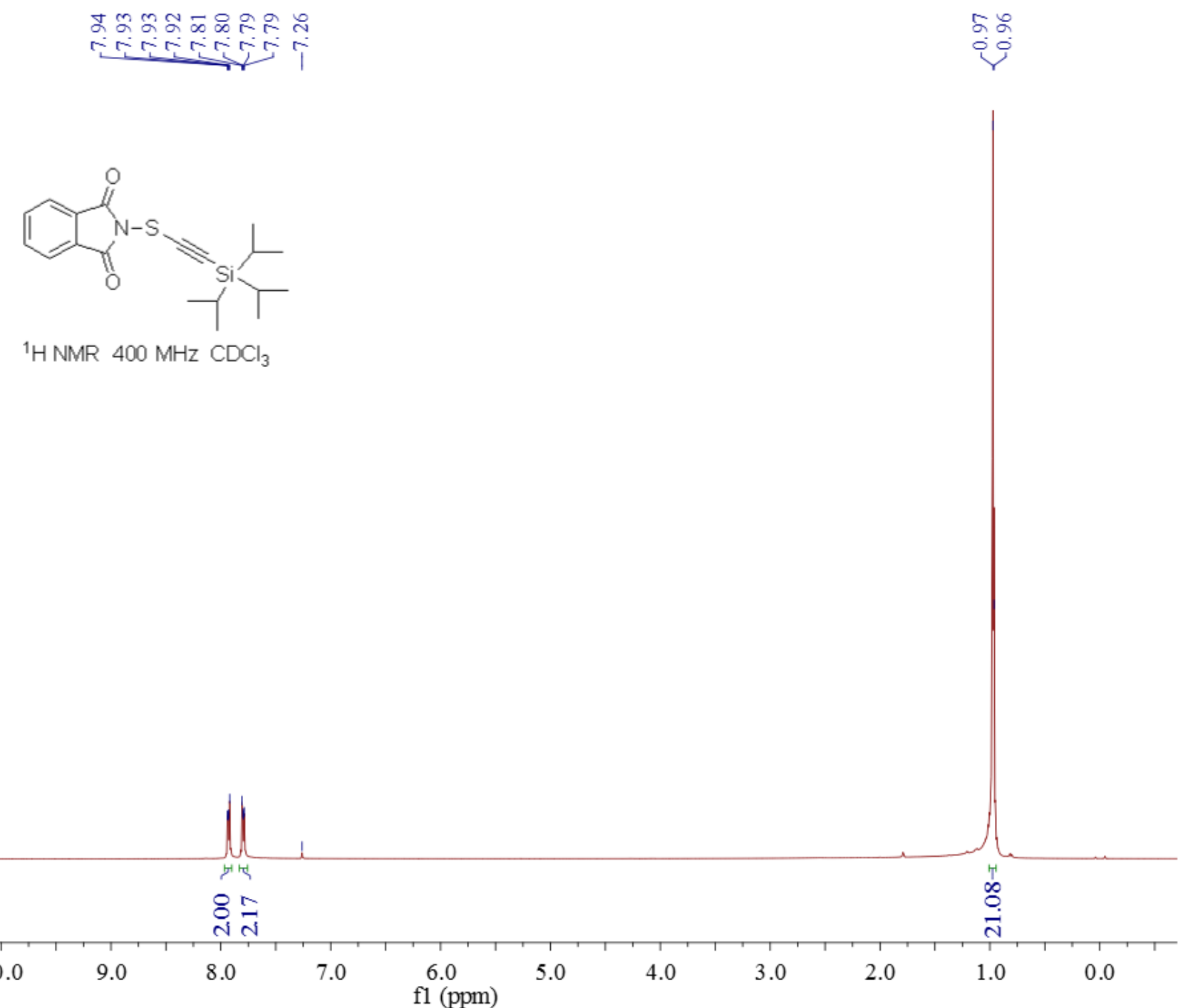



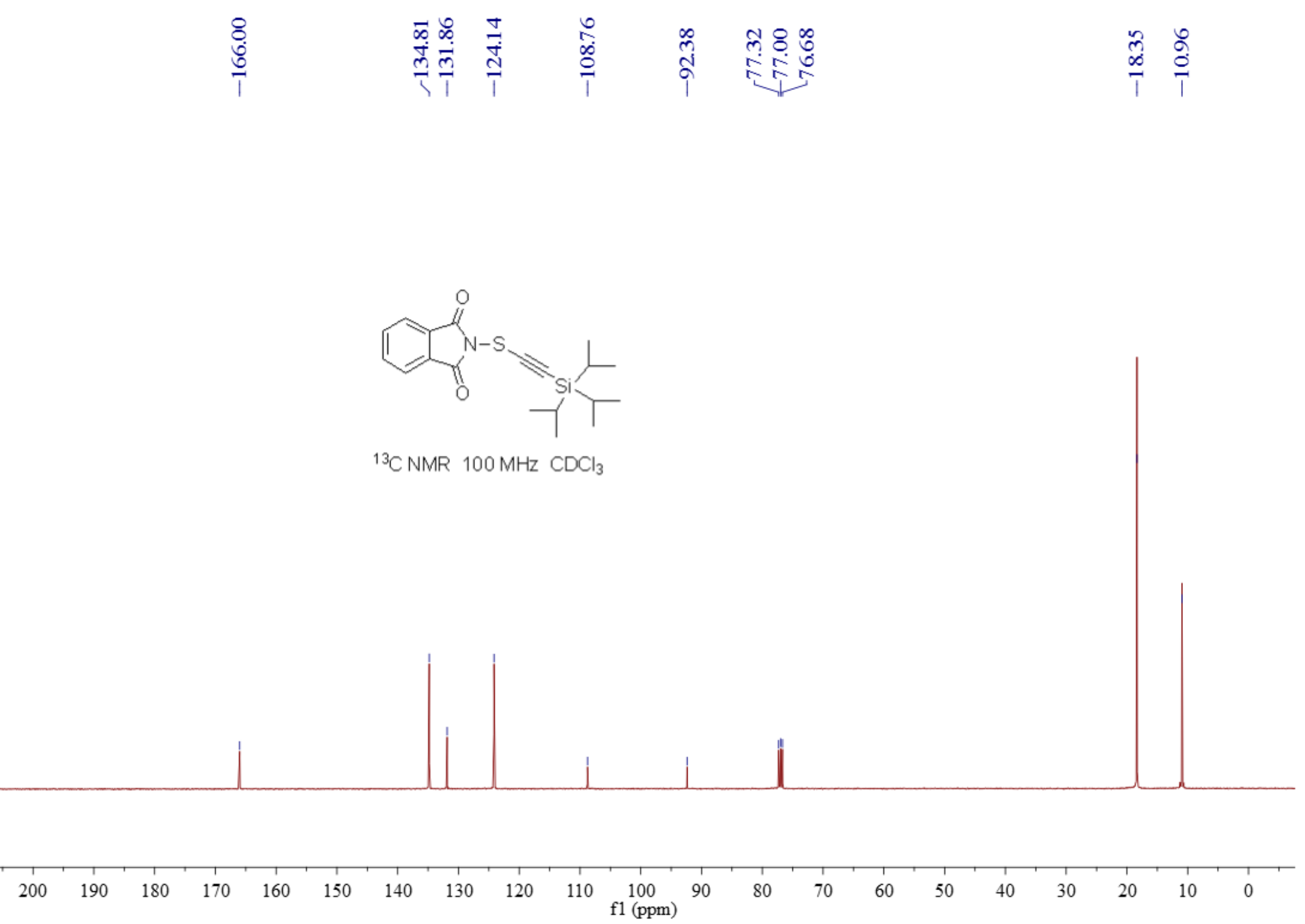

${ }^{13} \mathrm{CNMR} 100 \mathrm{MHz} \mathrm{CDCl}_{3}$

$\stackrel{\infty}{1} \stackrel{0}{\circ}$ 

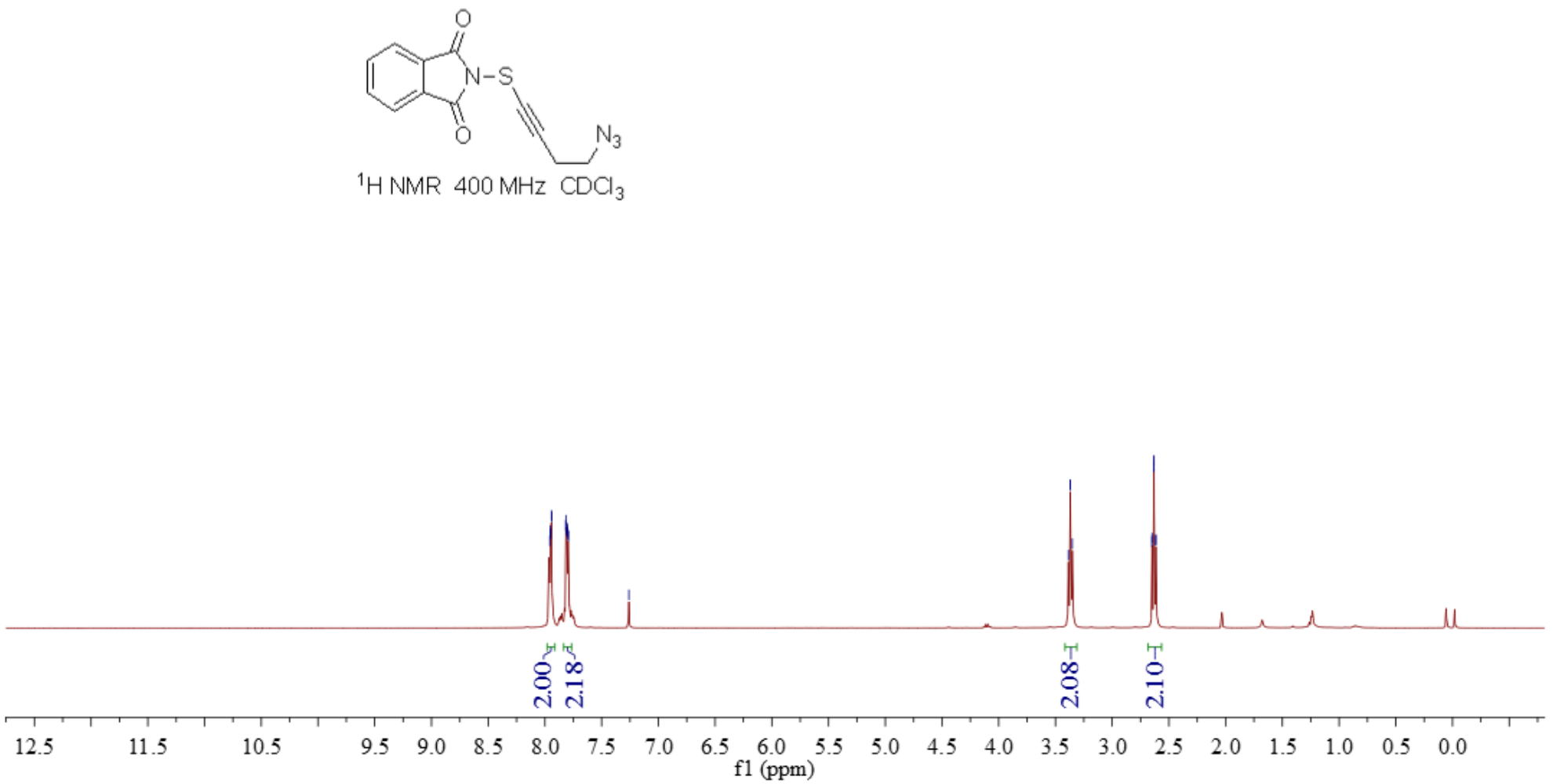


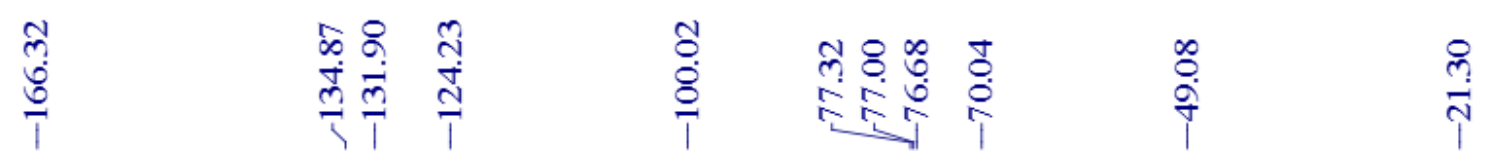
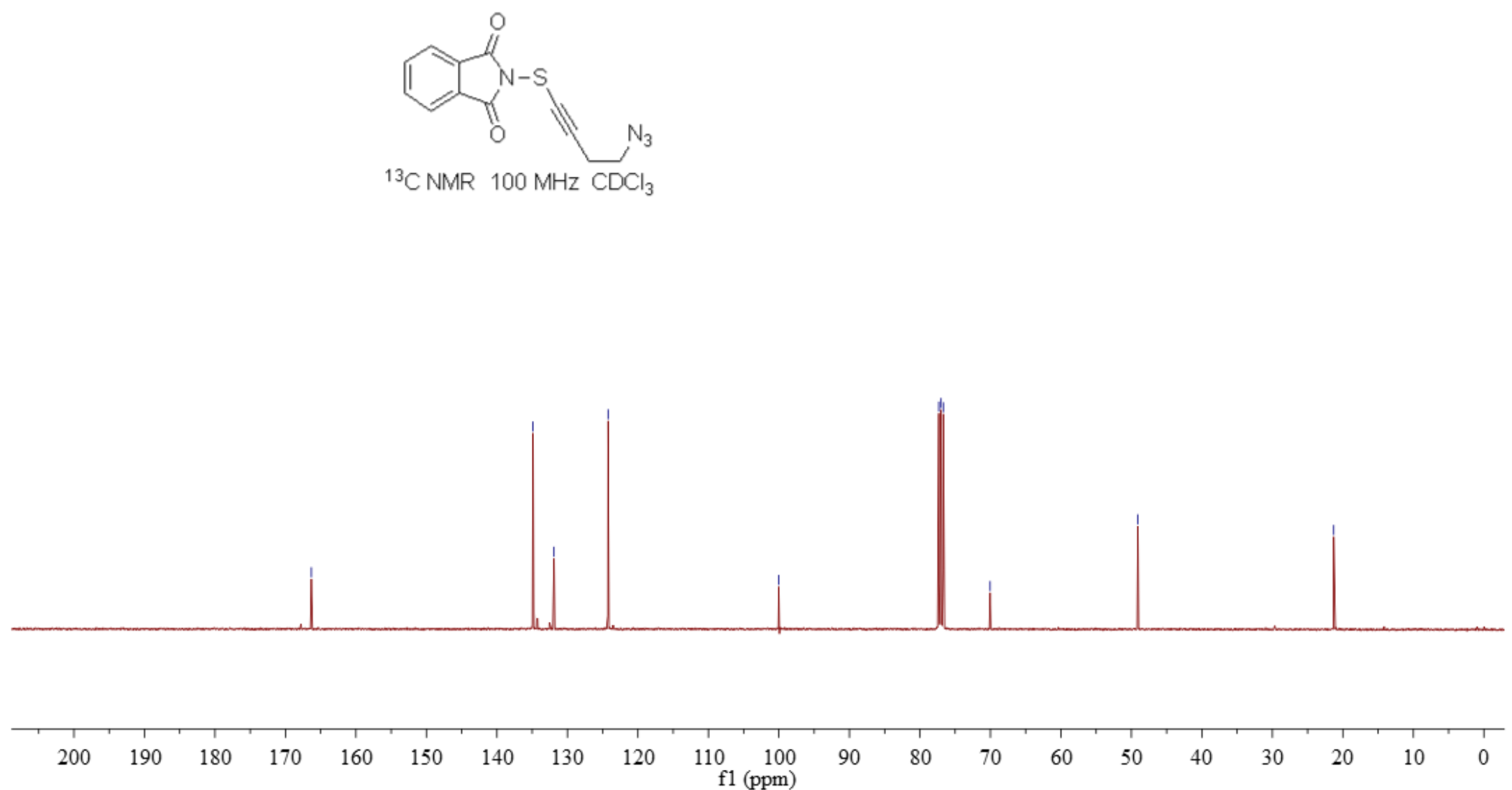

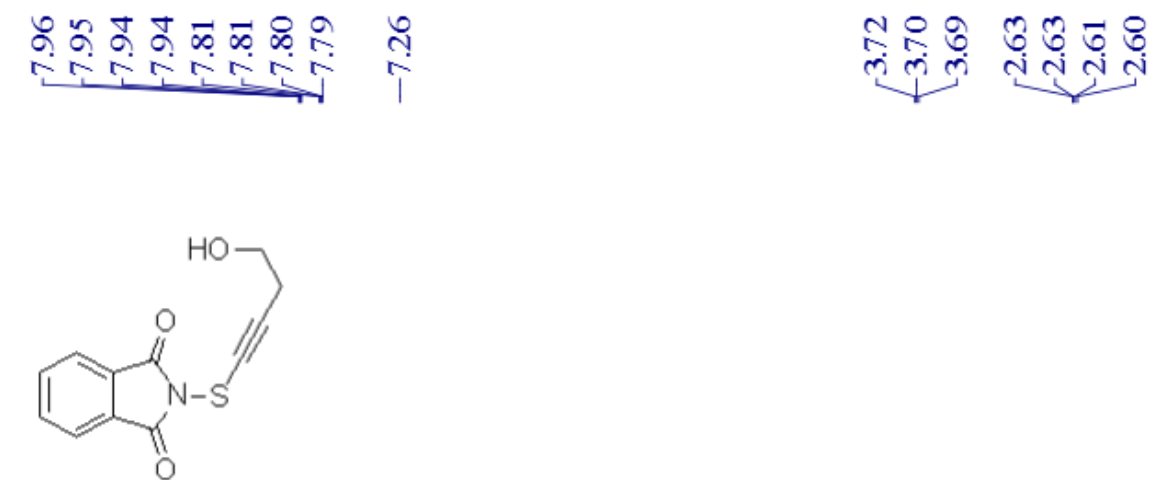

${ }^{1} \mathrm{HNMR} 400 \mathrm{MHz} \mathrm{CDCl}_{3}$

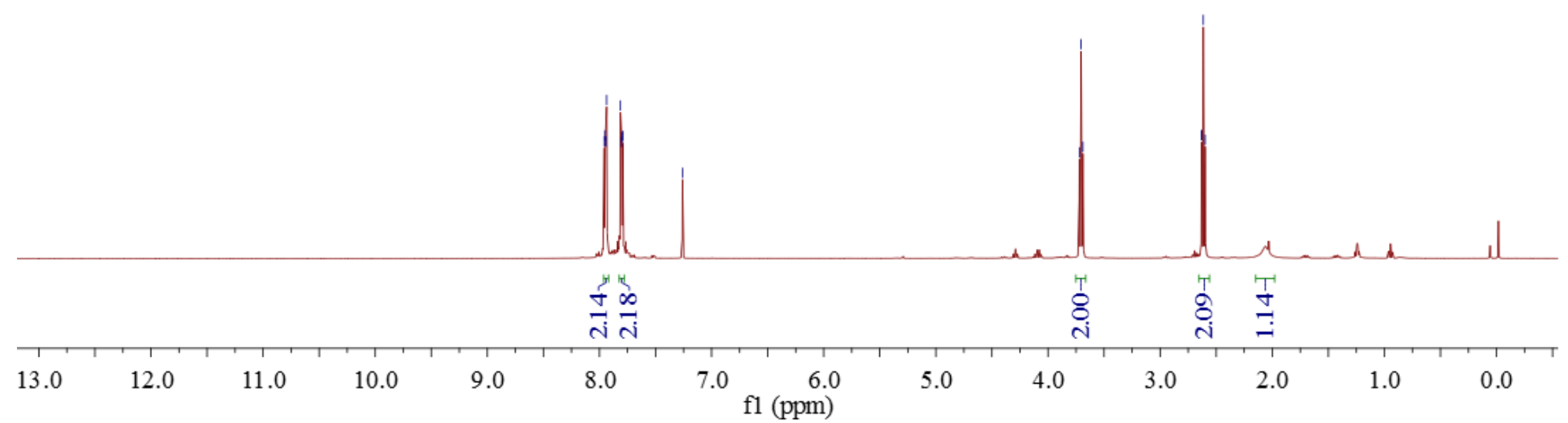




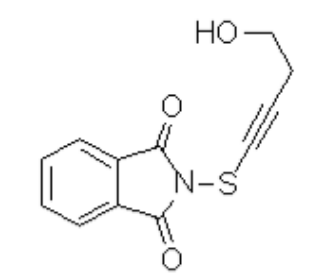

${ }^{13} \mathrm{CNMR} 100 \mathrm{MHz} \mathrm{CDCl}_{3}$
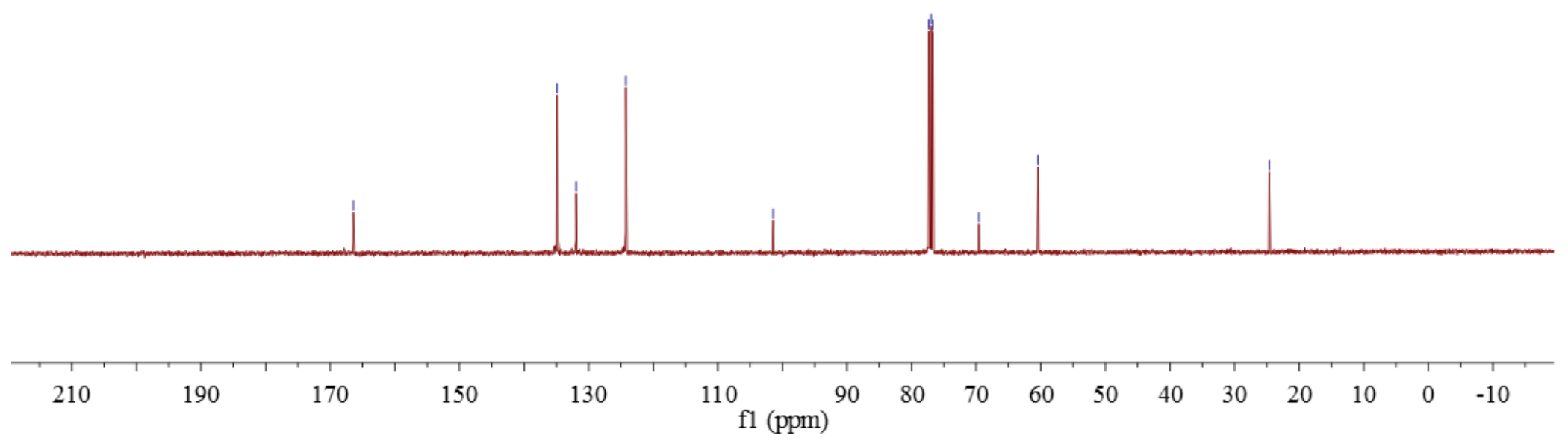


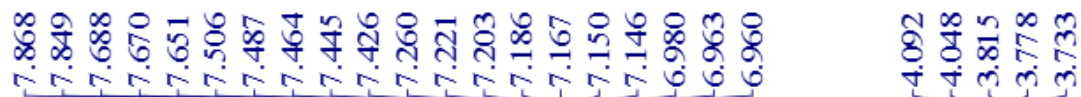

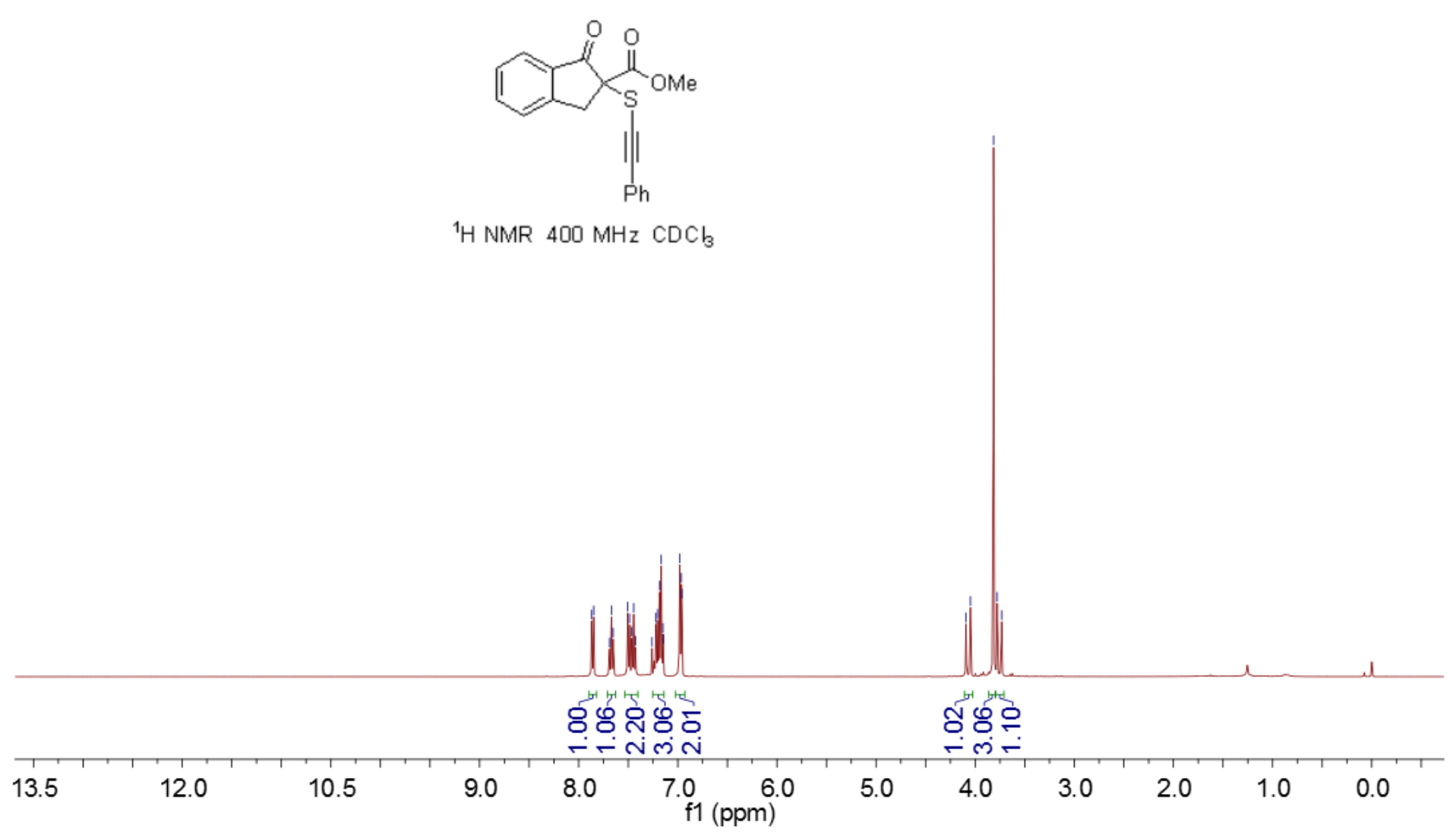




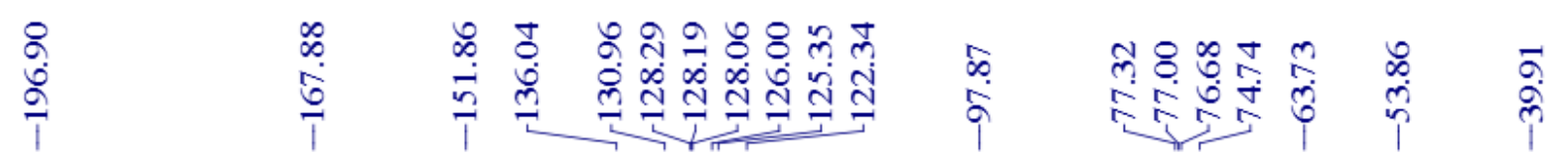

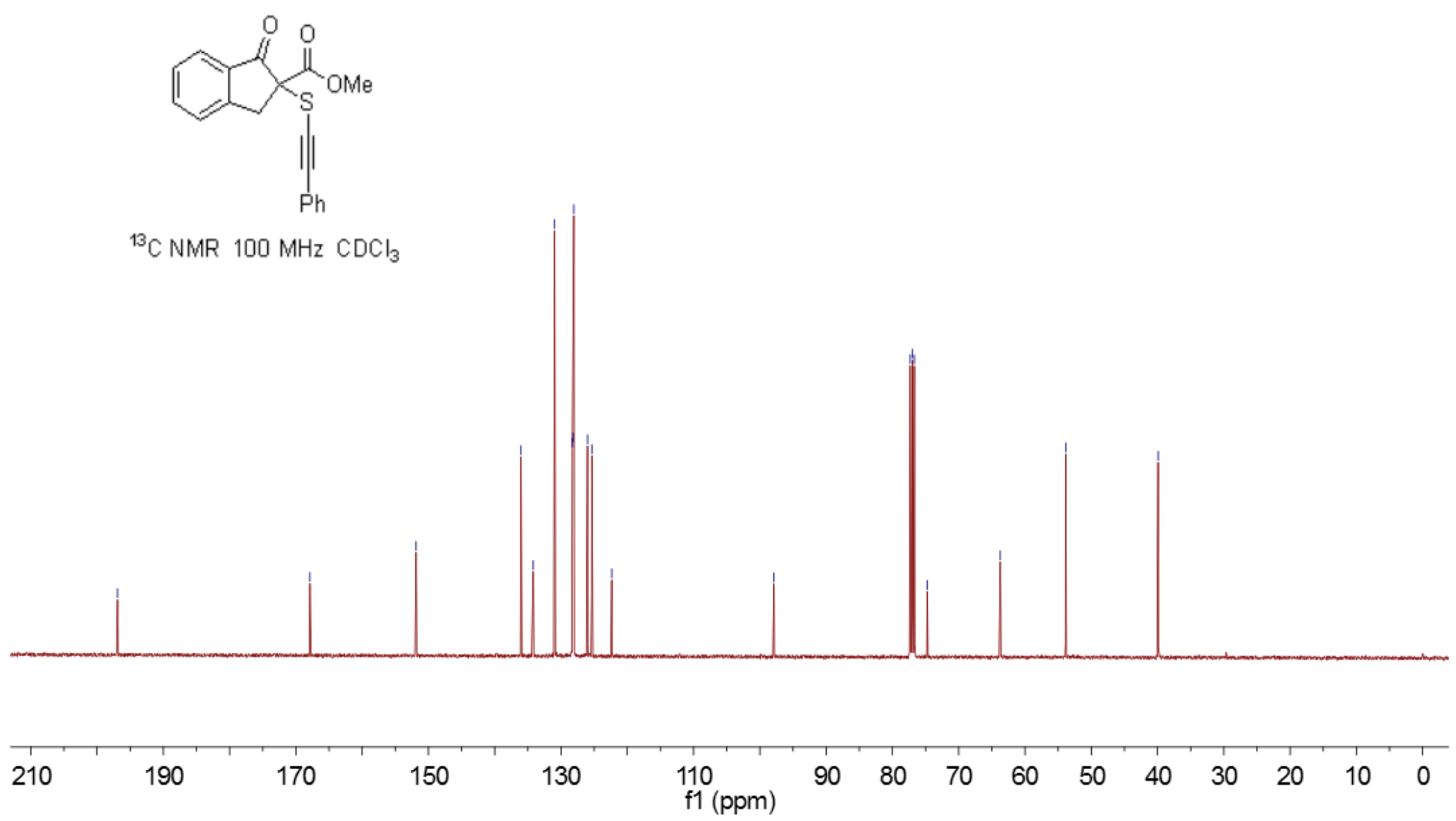


4b

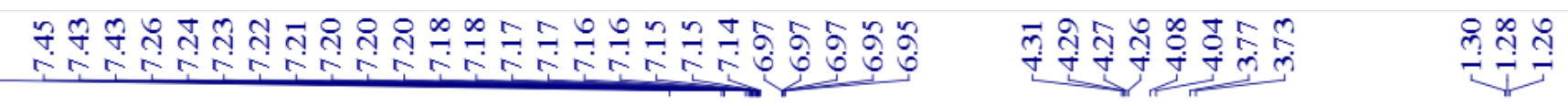

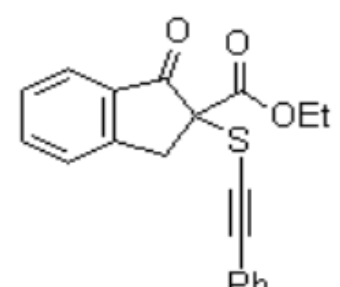

${ }^{1} \mathrm{H} \mathrm{NMR} \mathrm{CDCl}{ }_{3} 400 \mathrm{MHz}$

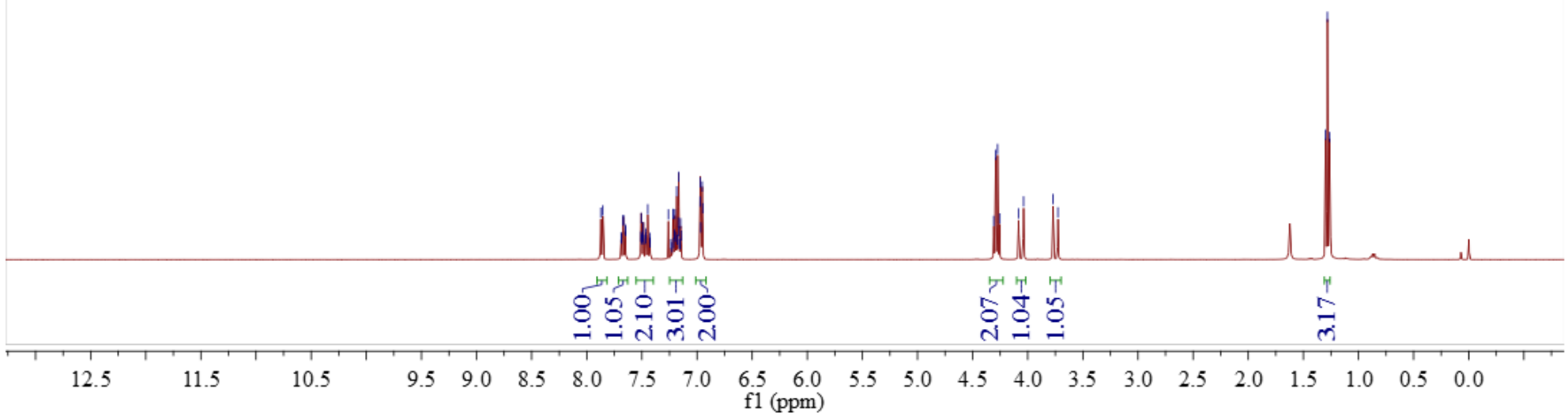




$$
\text { sull. }
$$




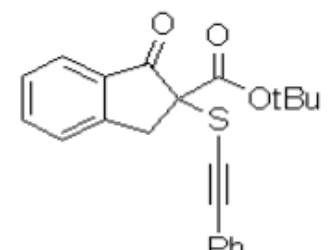

${ }^{1} \mathrm{HNMR} 400 \mathrm{MHz} \mathrm{CDCl}_{3}$

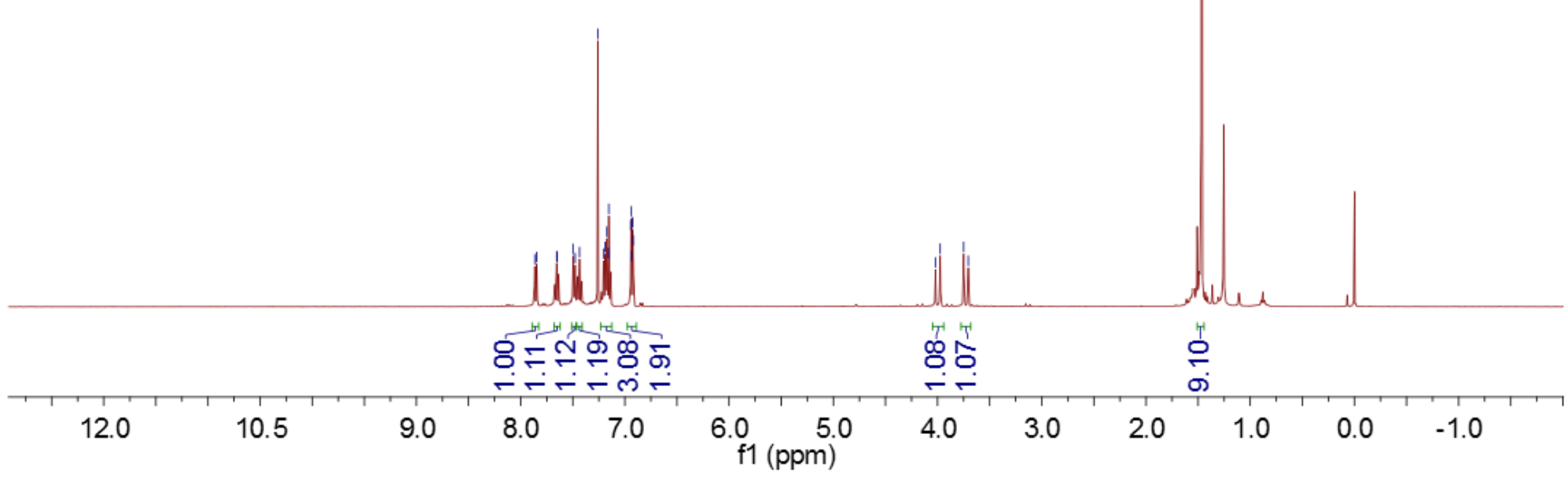




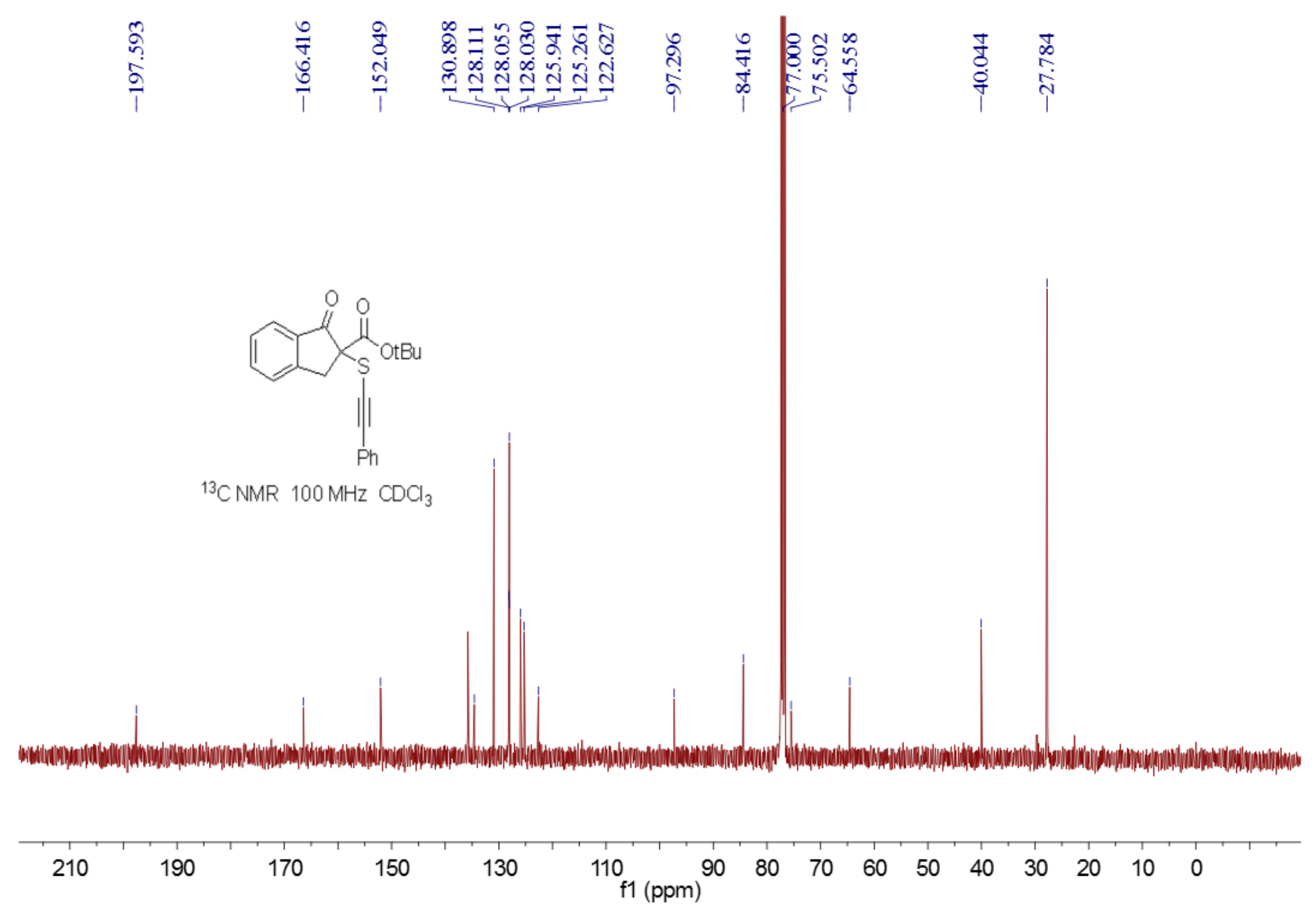




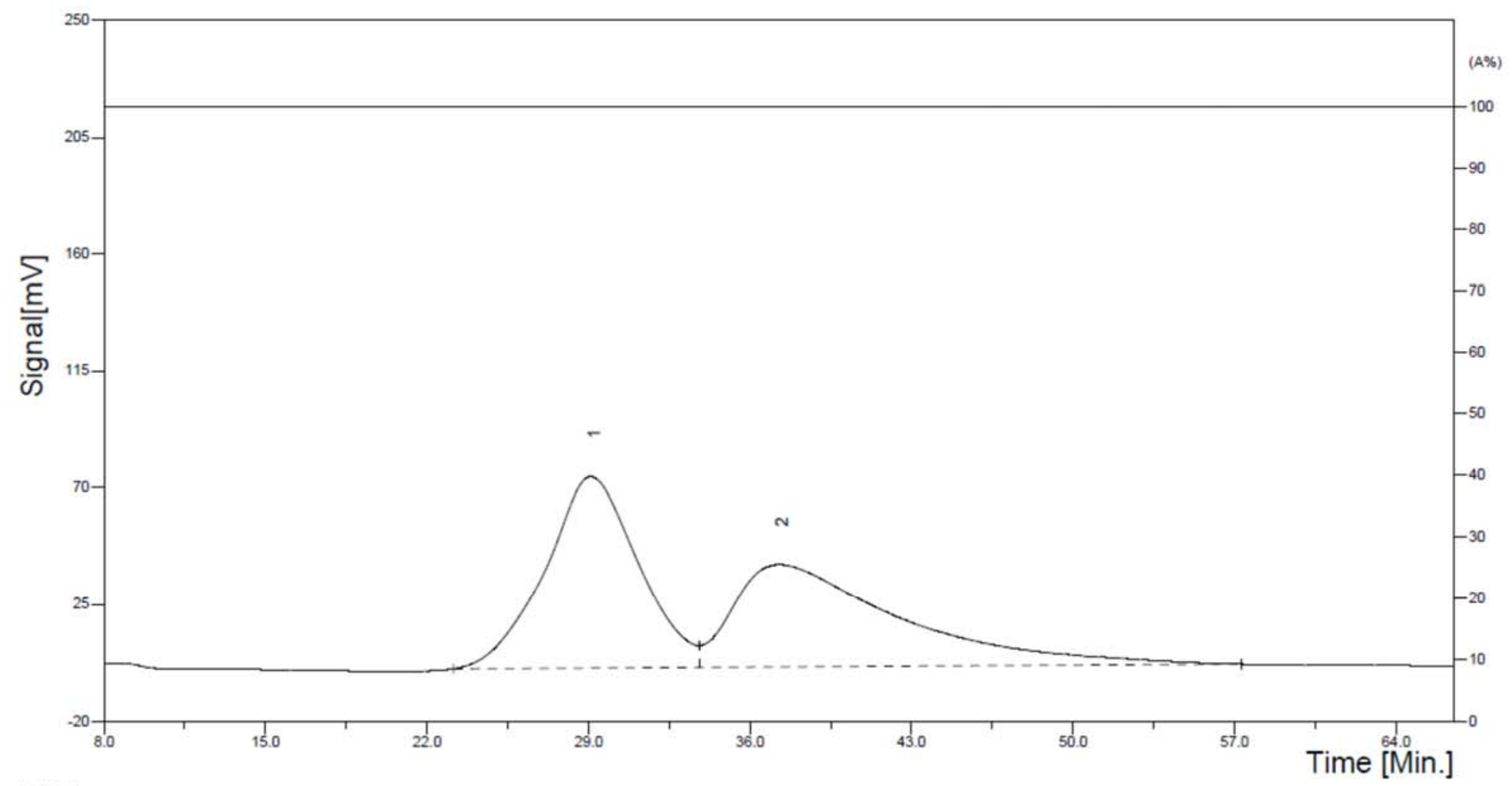

\begin{tabular}{|c|c|c|c|c|c|}
\hline$\#$ & 组分名 & 保留时间 (min) & 峰面积 (mV. sec) & 面积百分比 (\%) & 峰面积总和 (mv. sec) \\
\hline 1 & Unknown & 29. 10500 & 20717.79 & 51.2366 & 40435.56 \\
\hline 2 & Unknown & 37.25958 & 19717.77 & 48. 7634 & 40435.56 \\
\hline
\end{tabular}




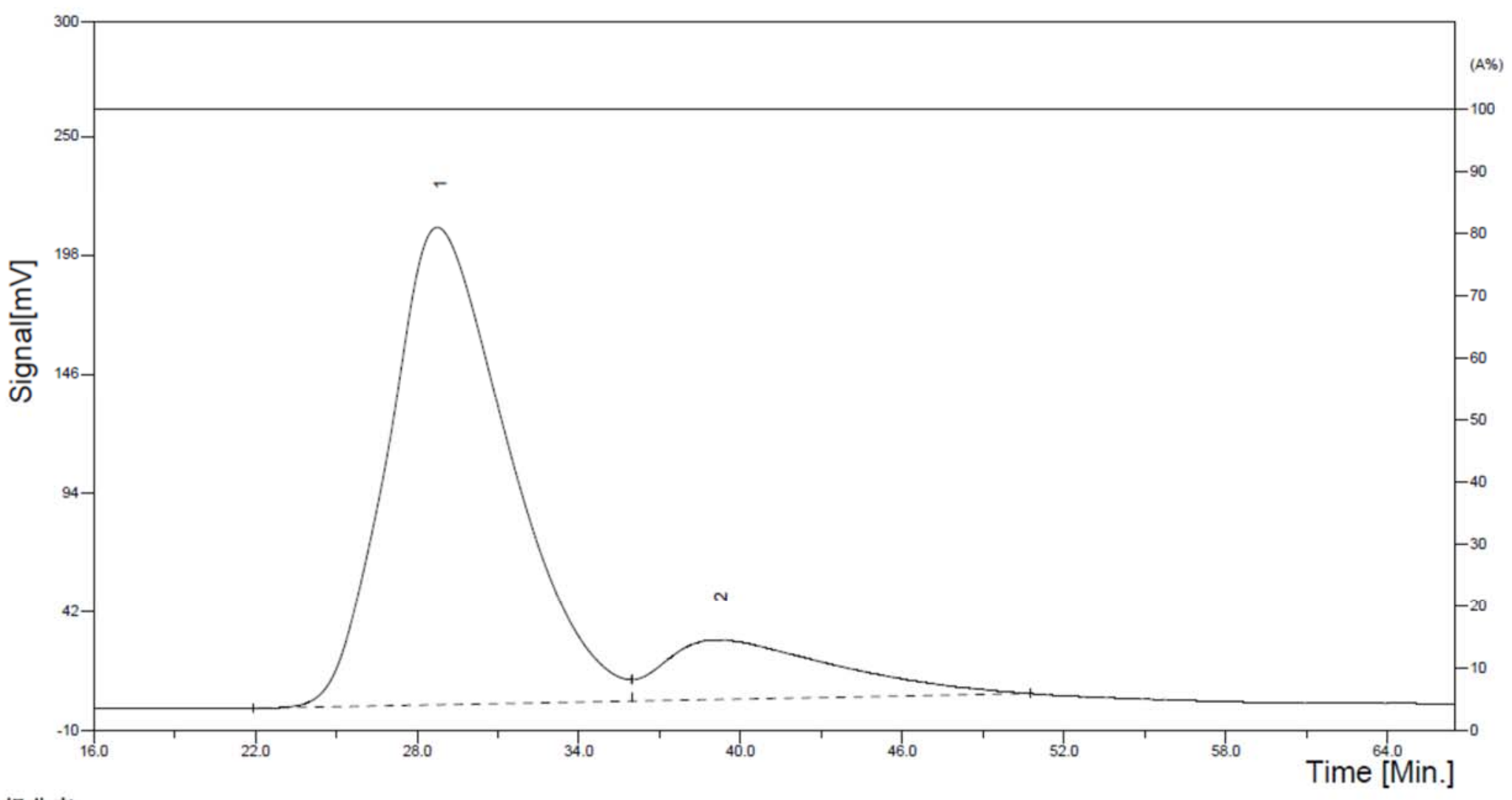

\begin{tabular}{|rlllll|}
\hline \multicolumn{1}{|l}{ 组分表 } & \multicolumn{1}{l|}{} \\
\hline \# & 组分名 & 保留时间 (min) & 峰高 (mV) & 峰面积 (mV. sec) & 面积百分比 (\%) \\
\hline 1 & Unknown & 28.75417 & 208.83 & 64974.82 & 84.9466 \\
2 & Unknown & 39.15833 & 25.98 & 11514.18 & 15.0534 \\
\hline
\end{tabular}




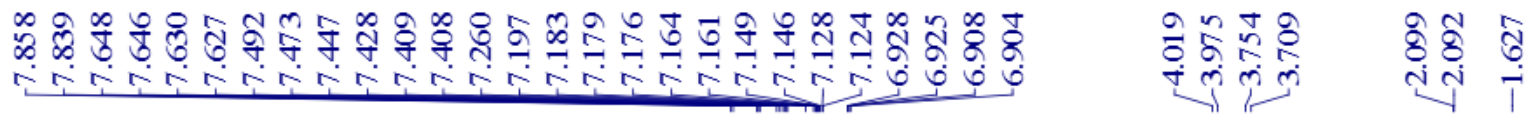

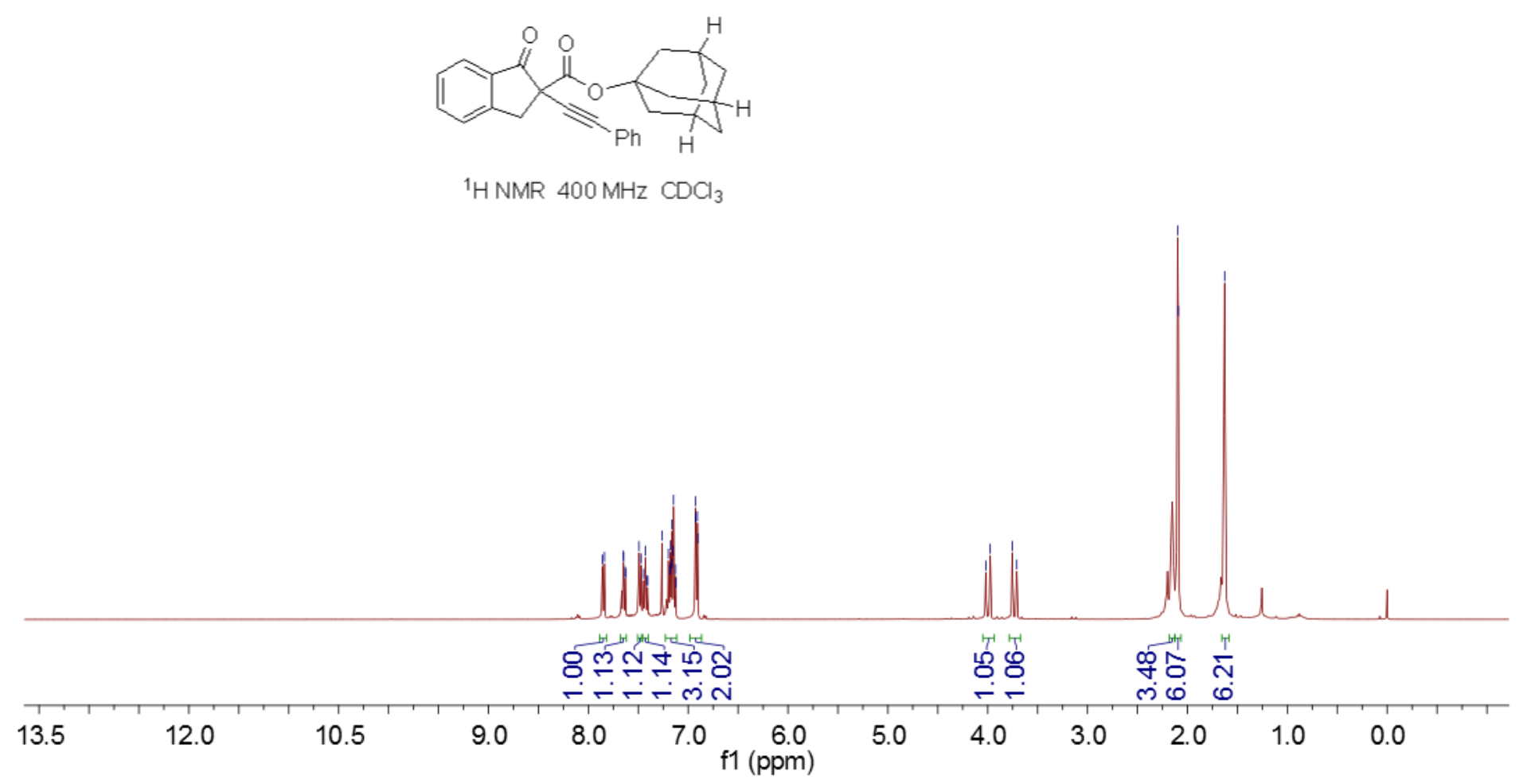




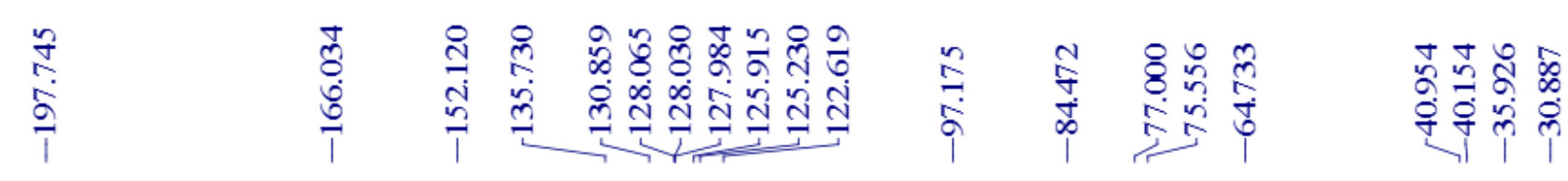

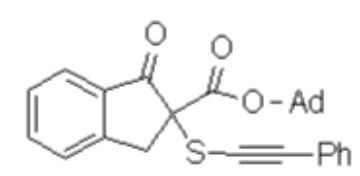

${ }^{13} \mathrm{CNMR} 100 \mathrm{MHz} \mathrm{CDCl}_{3}$

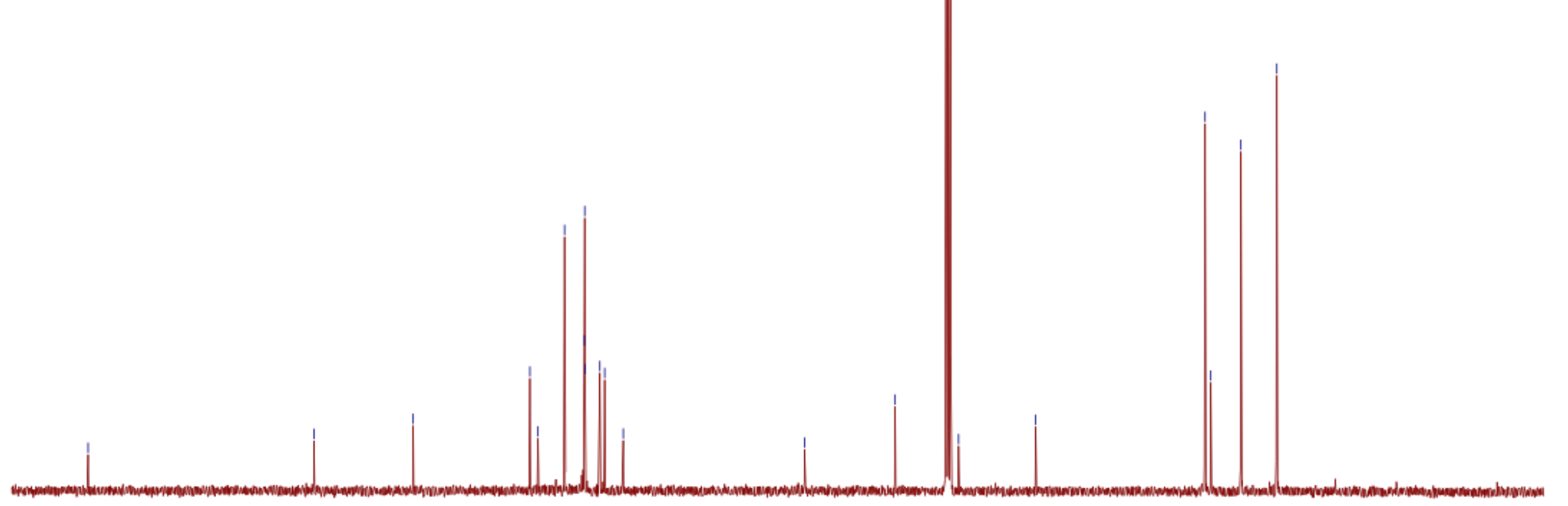

190

170

150

130

110 f1 (ppm)

$90 \quad 80$

70

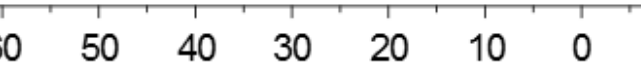




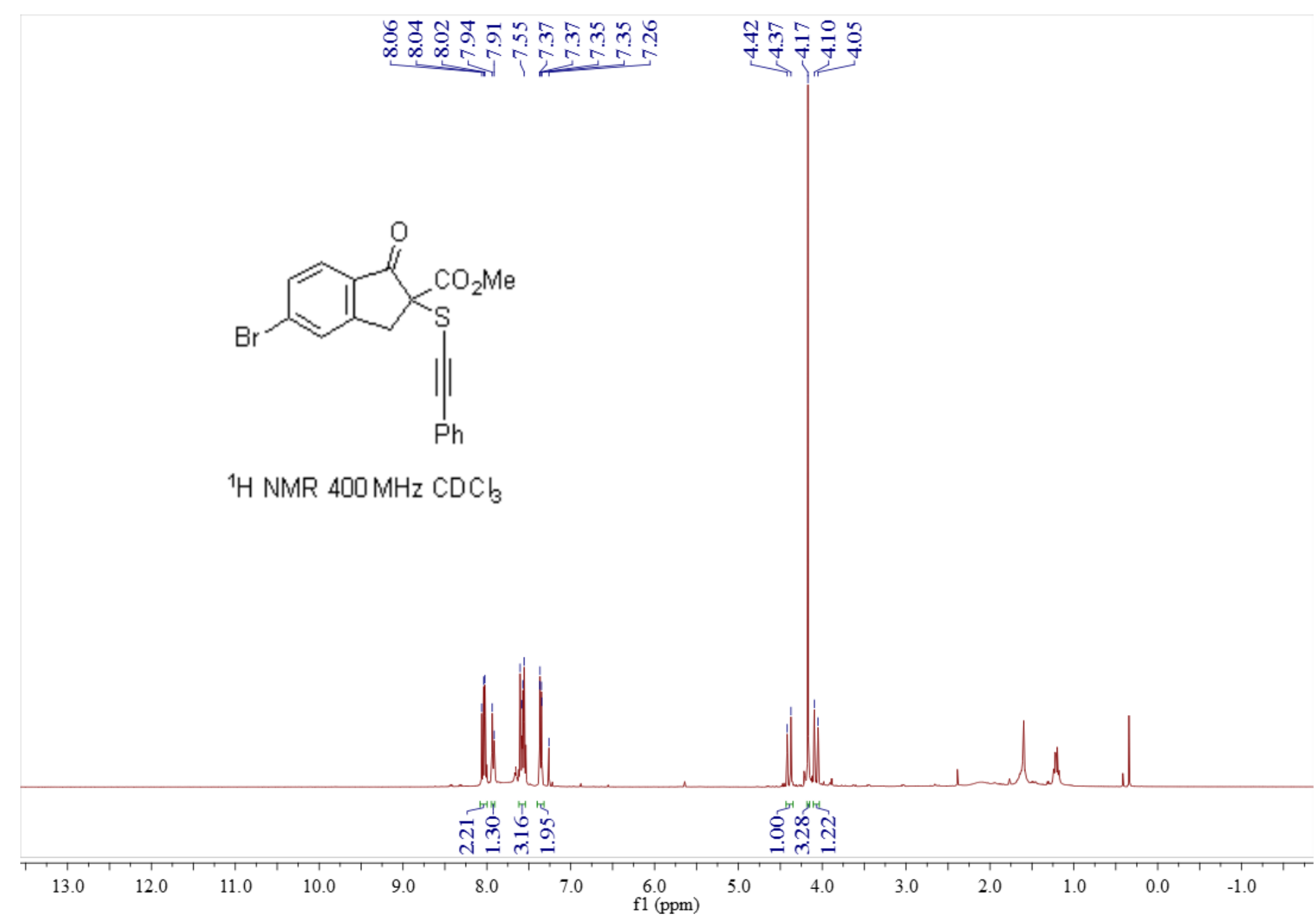




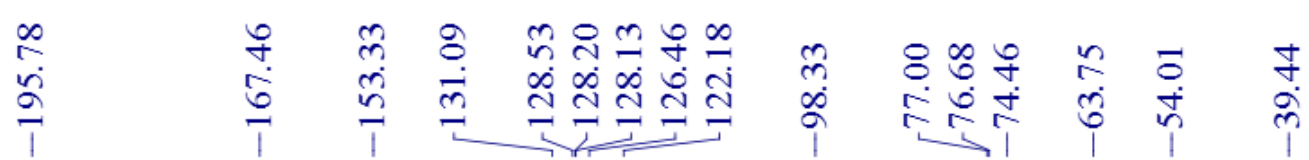

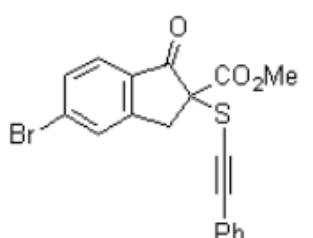

${ }^{13} \mathrm{C} \mathrm{NMR} 100 \mathrm{MHz} \mathrm{CDCl}_{3}$

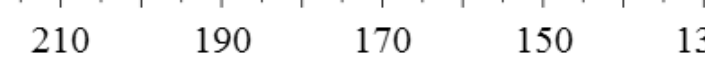

30 


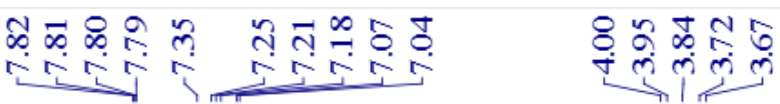

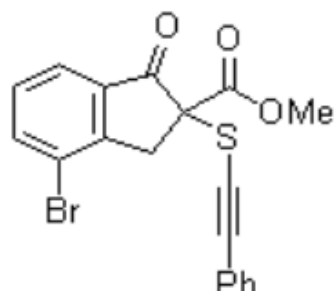

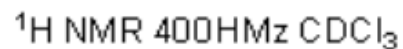

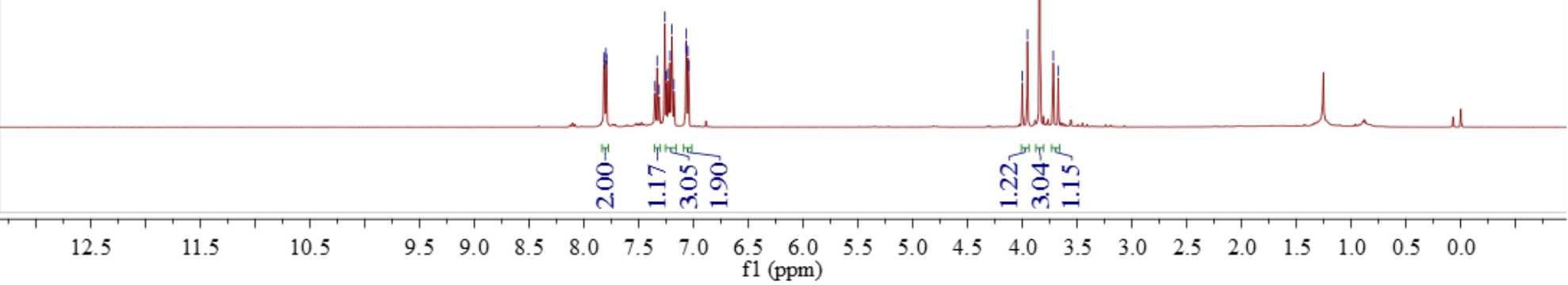

f1 (ppm) 


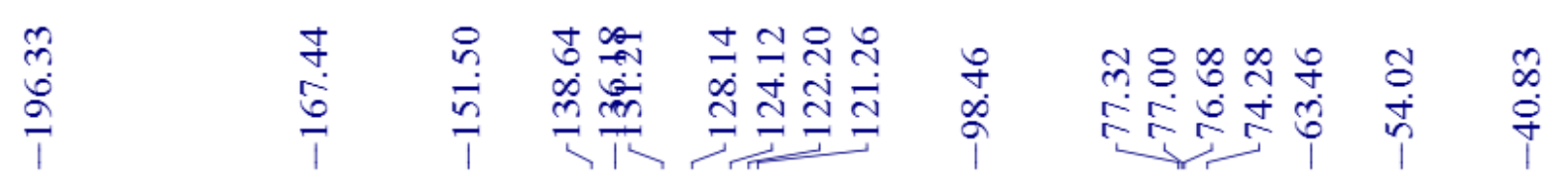

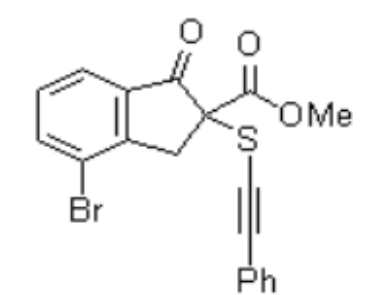

${ }^{13} \mathrm{C} \mathrm{NMR}_{100 \mathrm{Mz}} \mathrm{CDCl}_{3}$
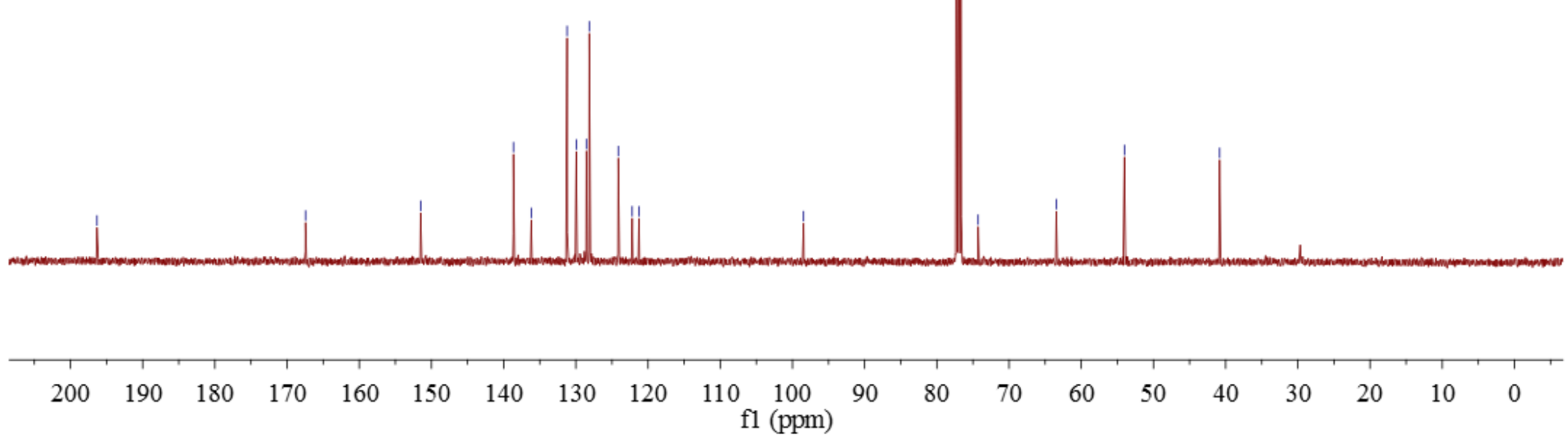


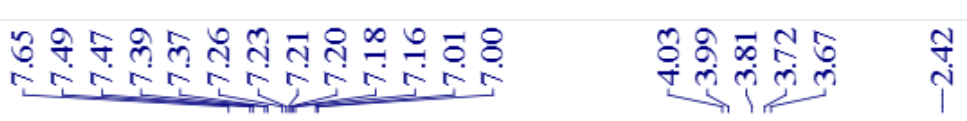

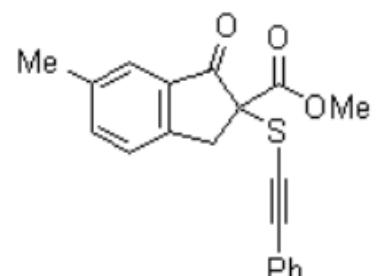

${ }^{1} \mathrm{H} \mathrm{NMR} 400 \mathrm{MHz} \mathrm{CDCl}_{3}$

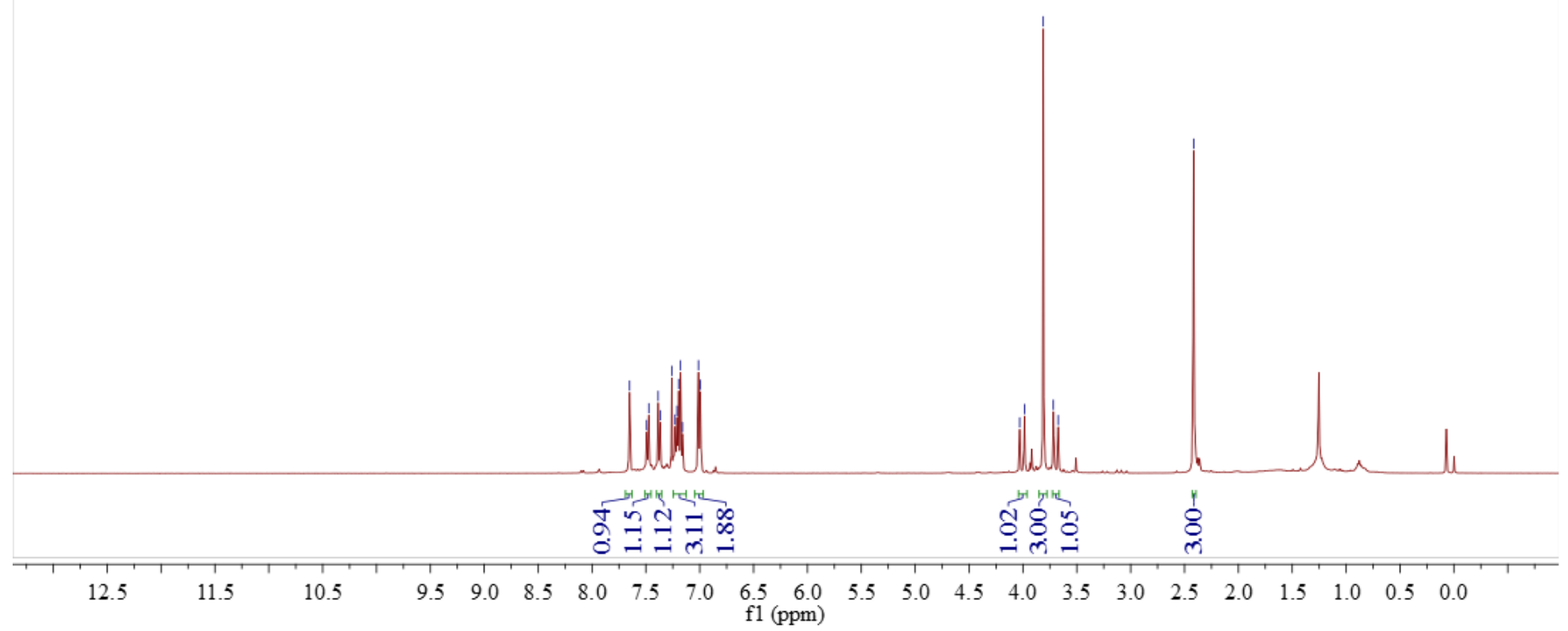




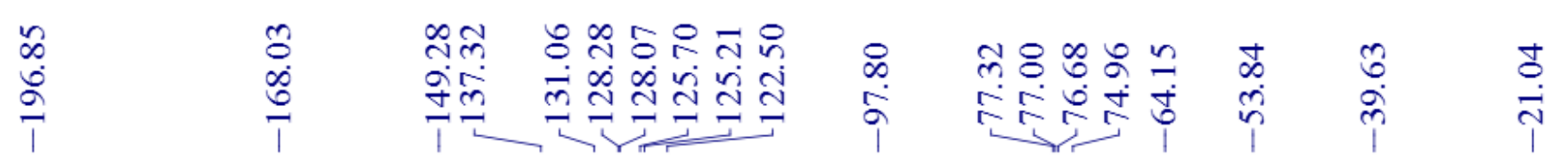

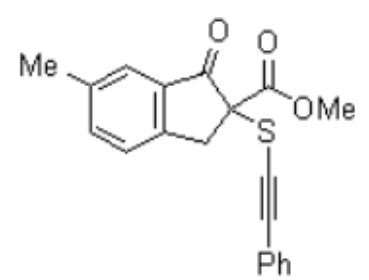

${ }^{13} \mathrm{C} \mathrm{NMR} 100 \mathrm{MHz} \mathrm{CDCl}_{3}$

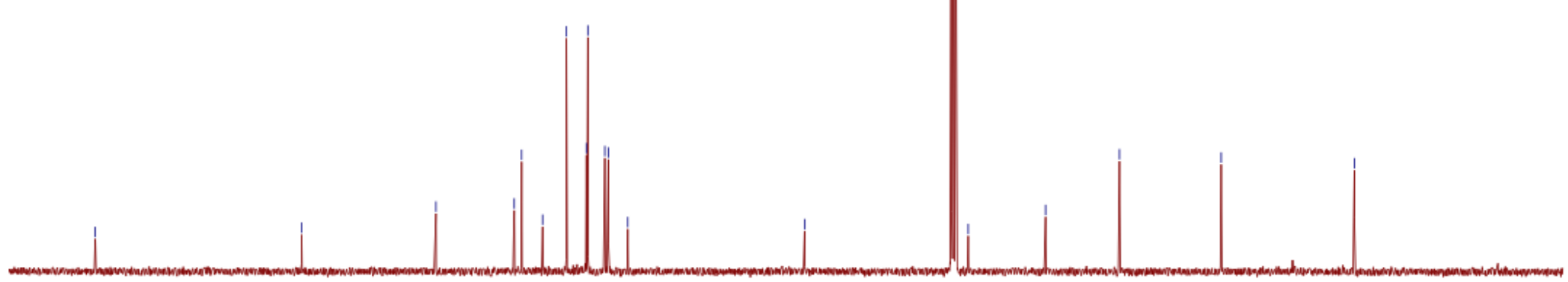

$\begin{array}{lllllllllllllllllllll}200 & 190 & 180 & 170 & 160 & 150 & 140 & 130 & 120 & 110 \underset{\mathrm{fl}(\mathrm{ppm})}{100} & 90 & 80 & 70 & 60 & 50 & 40 & 30 & 20 & 10 & 0\end{array}$ 


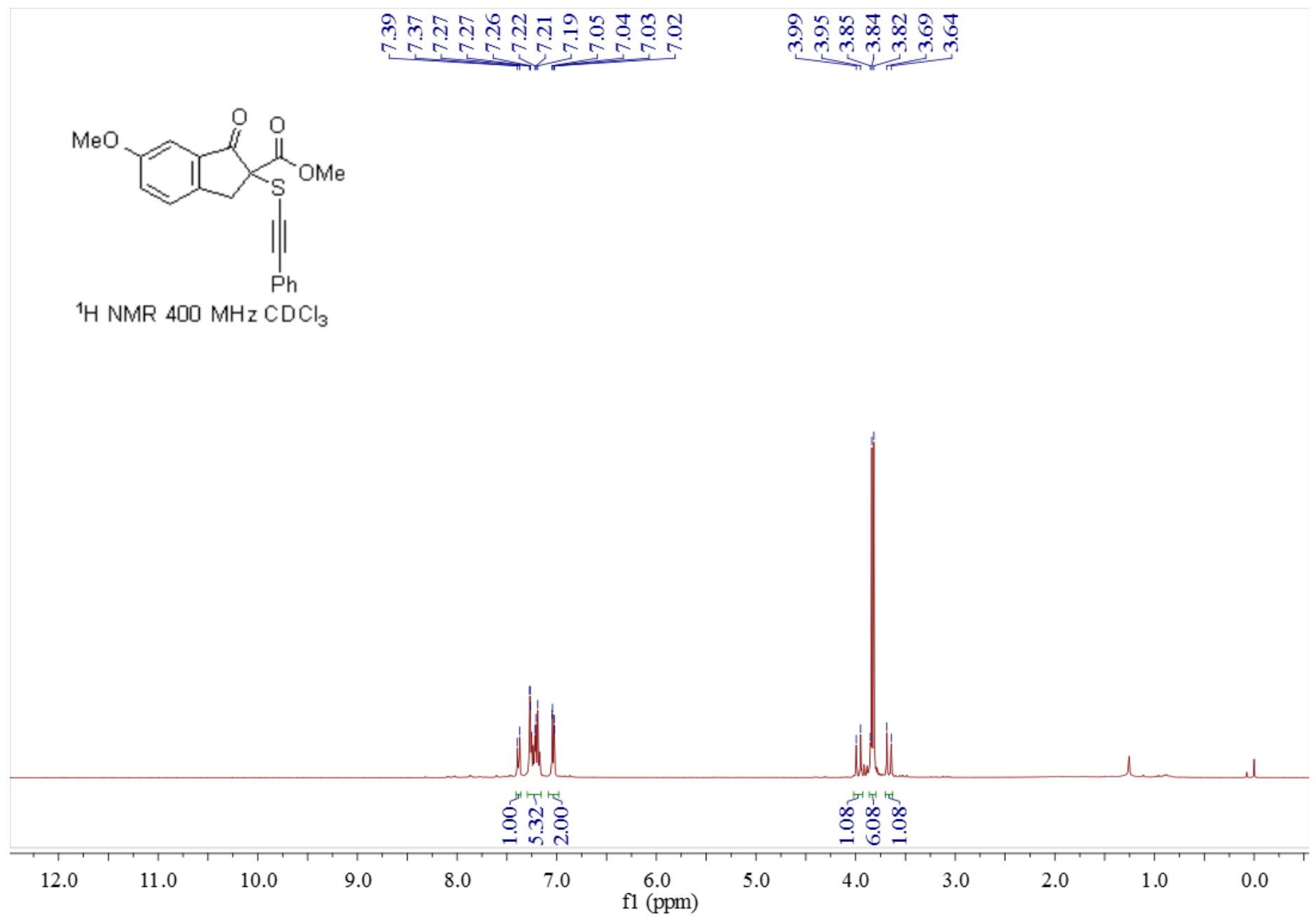




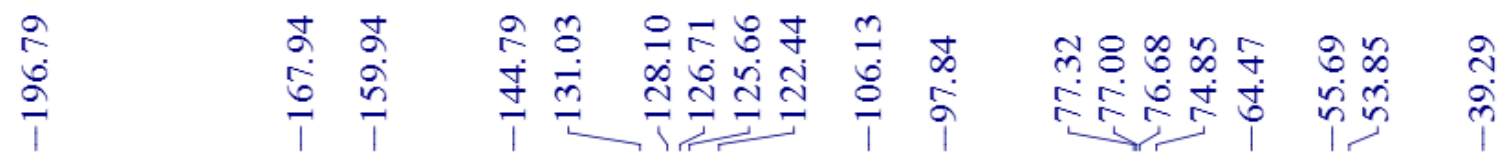

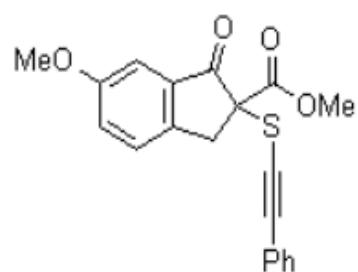

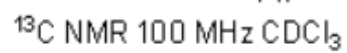

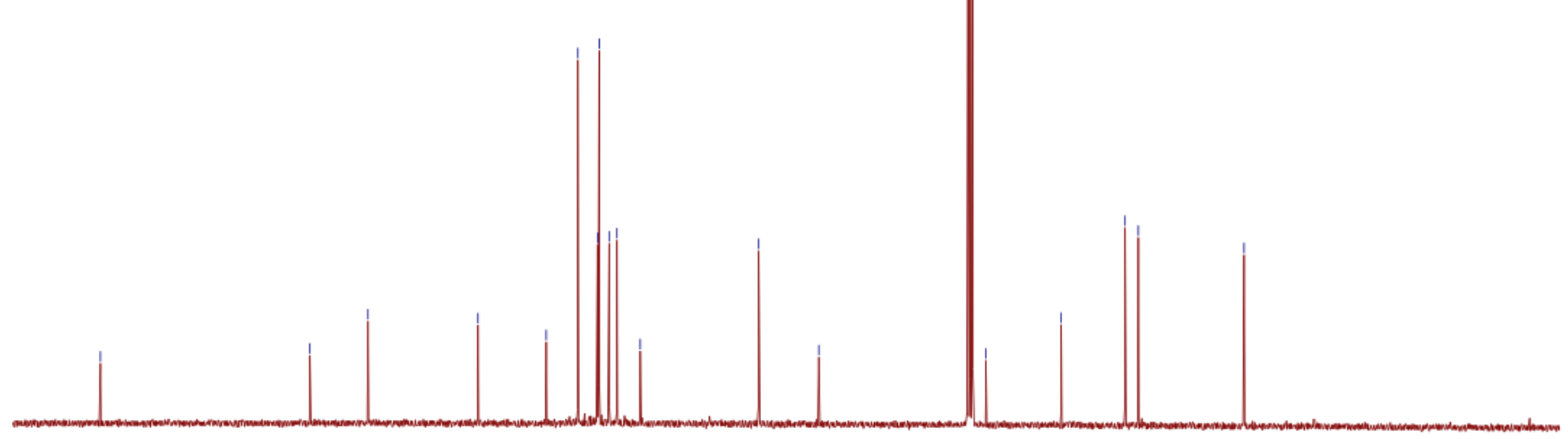

$\begin{array}{lllllllllllllllllllll}200 & 190 & 180 & 170 & 160 & 150 & 140 & 130 & 120 & \begin{array}{c}110 \\ \mathrm{fl}(\mathrm{ppm})\end{array} & 90 & 80 & 70 & 60 & 50 & 40 & 30 & 20 & 10 & 0\end{array}$ 


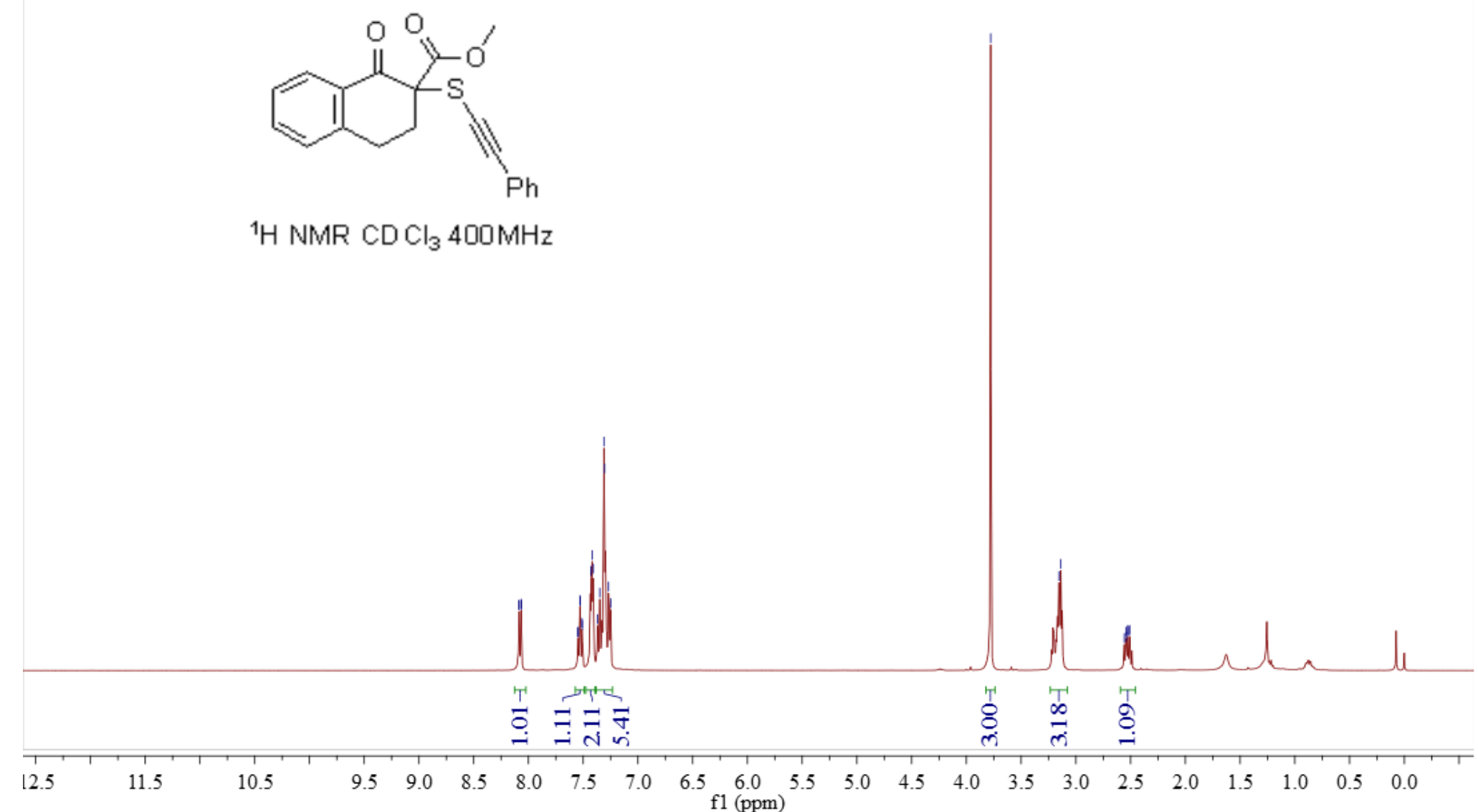




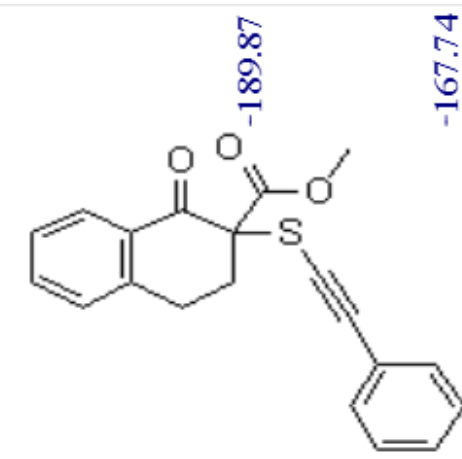

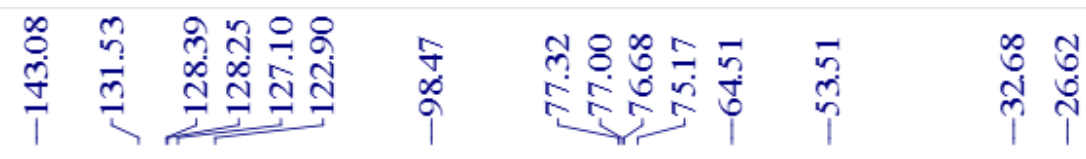

${ }^{13} \mathrm{C} \mathrm{NMR} \mathrm{CDCb} 100 \mathrm{MHz}$

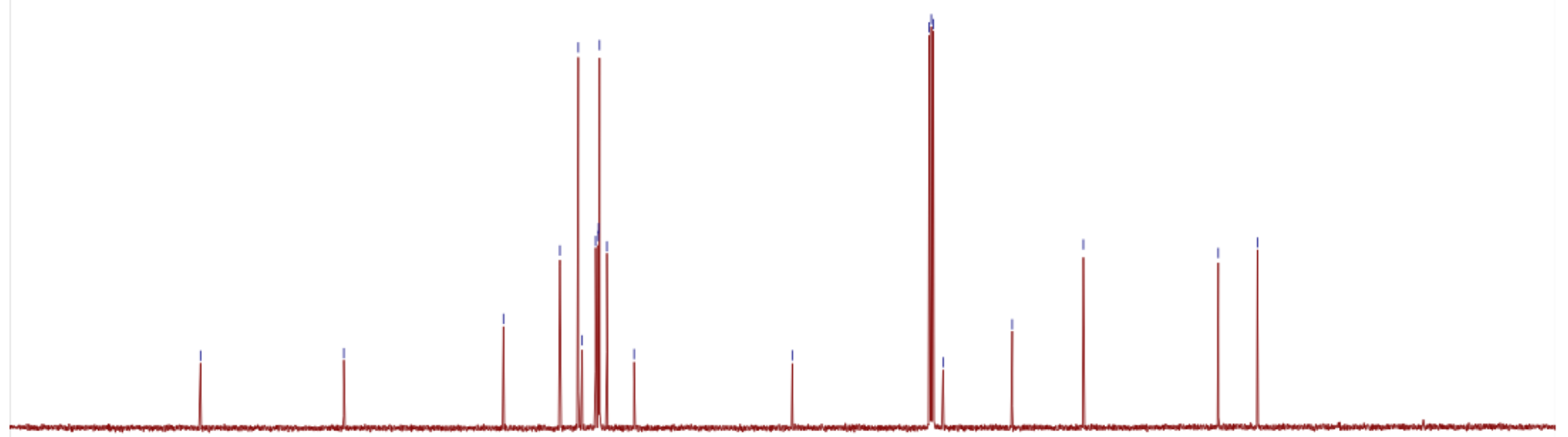

$\begin{array}{llllllllllllllllllllllll}210 & 200 & 190 & 180 & 170 & 160 & 150 & 140 & 130 & 120 & 110 & 100 & 90 & 80 & 70 & 60 & 50 & 40 & 30 & 20 & 10 & 0 & -10 & \end{array}$ 


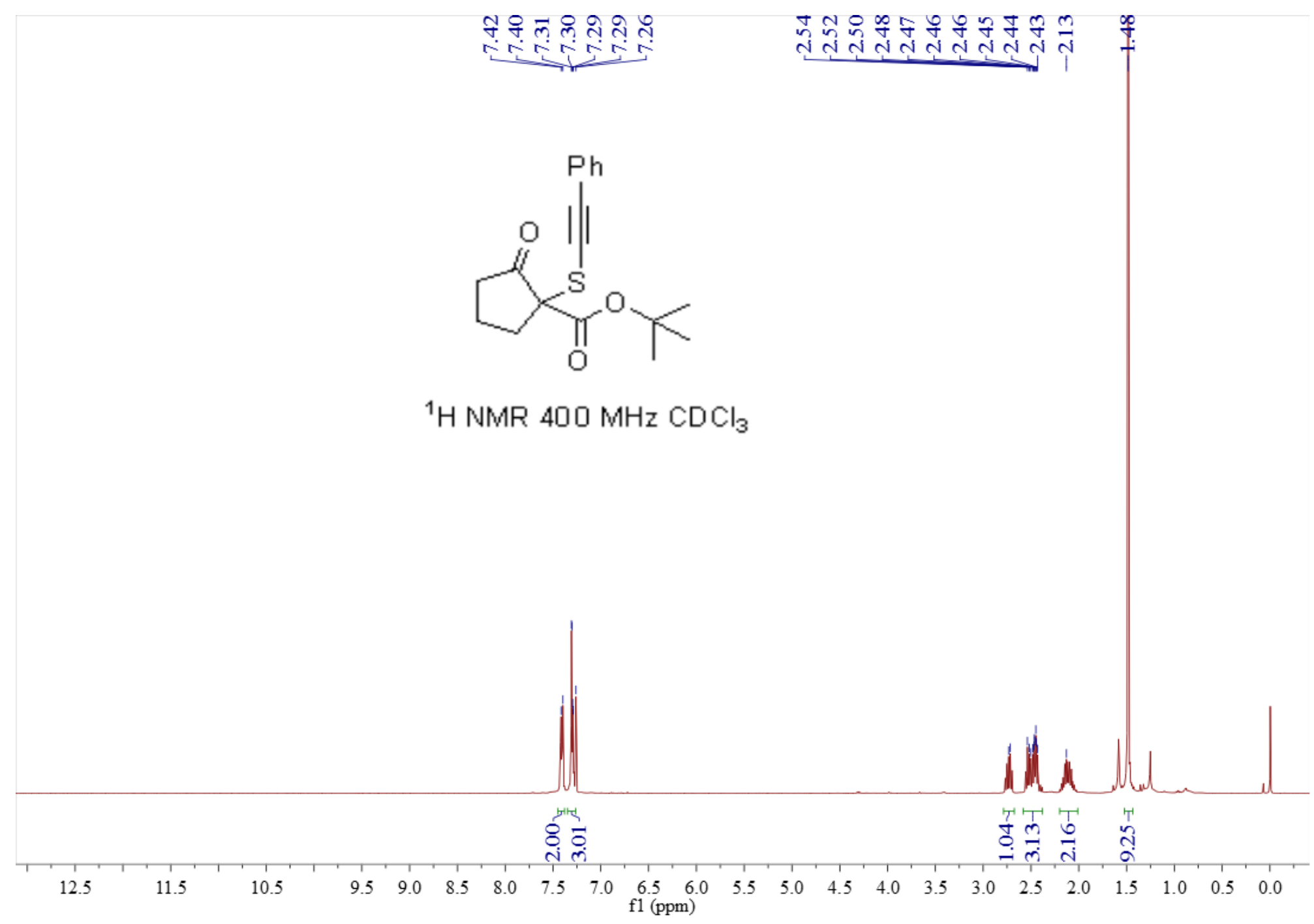




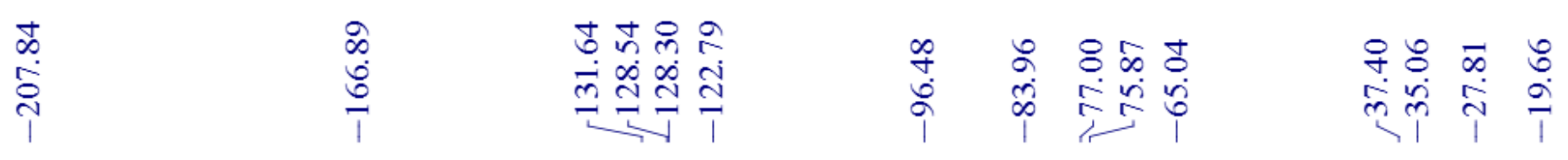

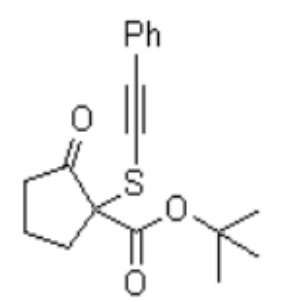

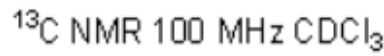
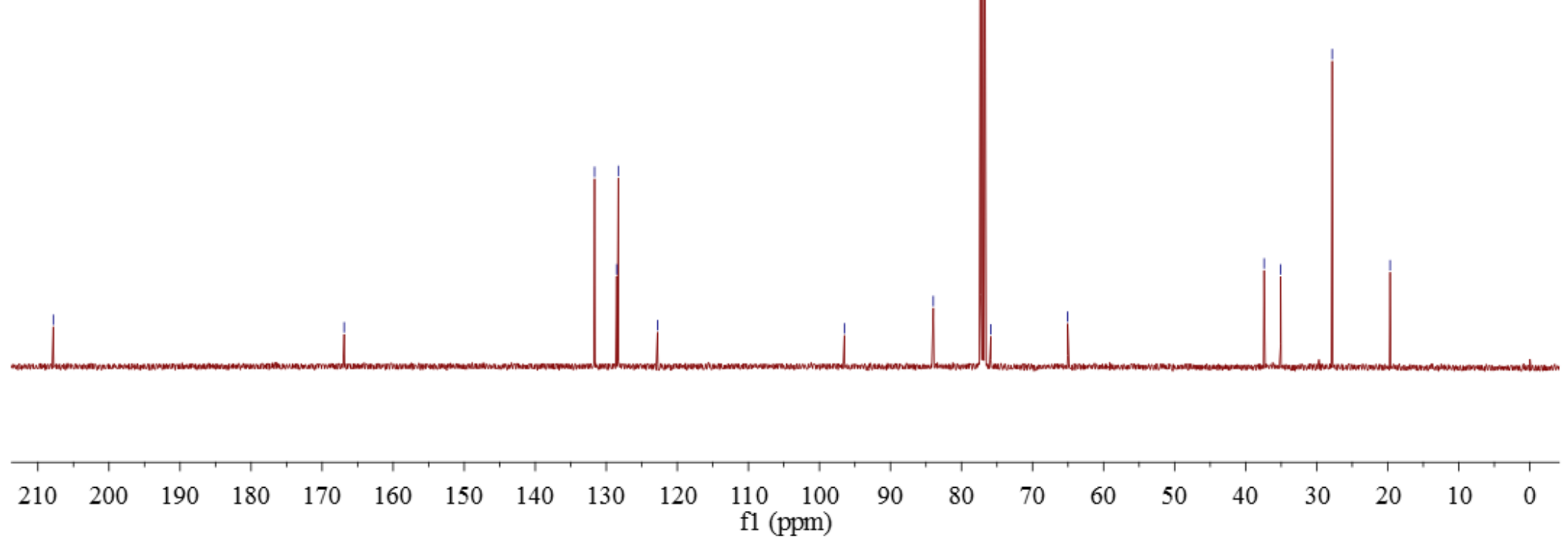


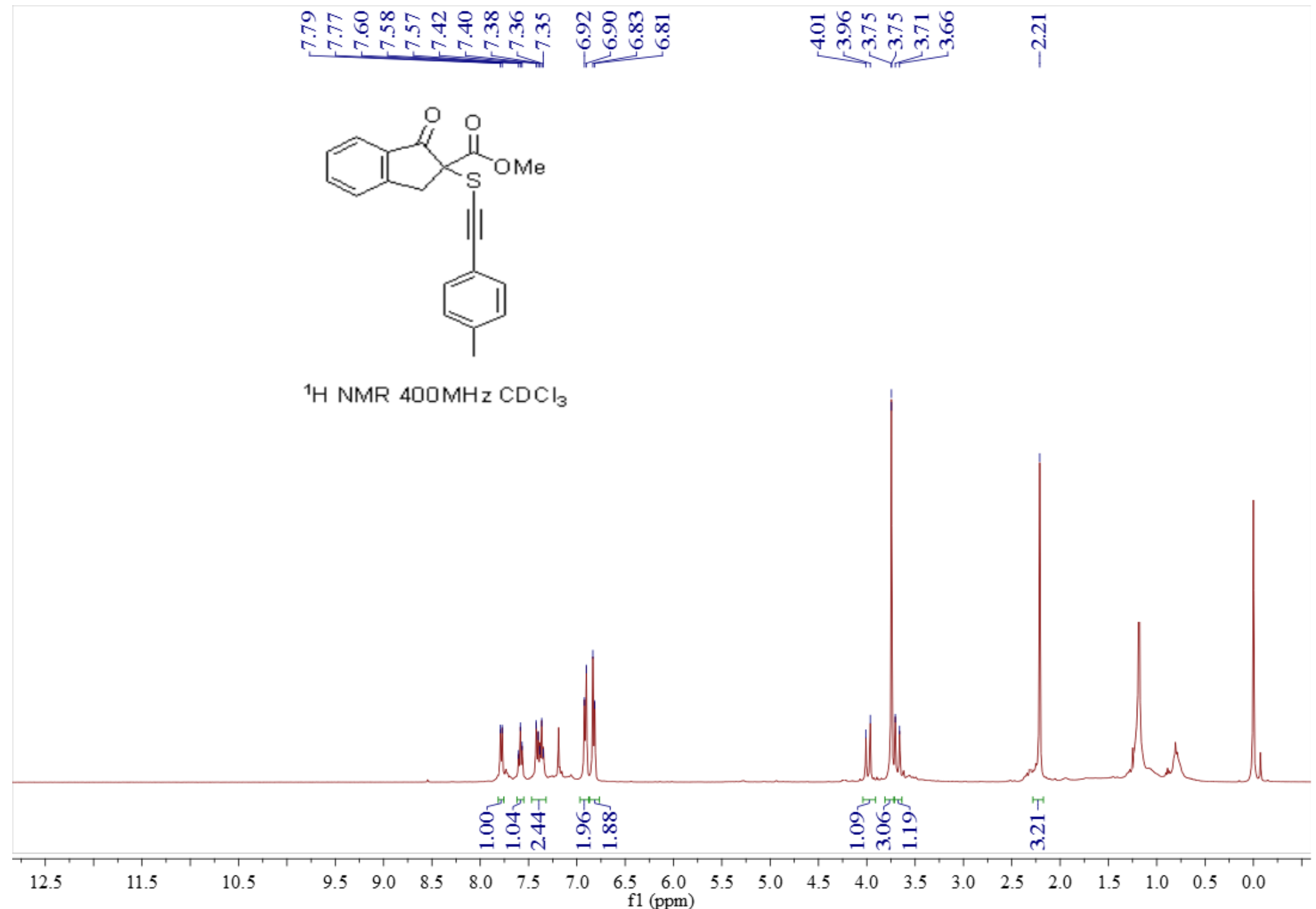



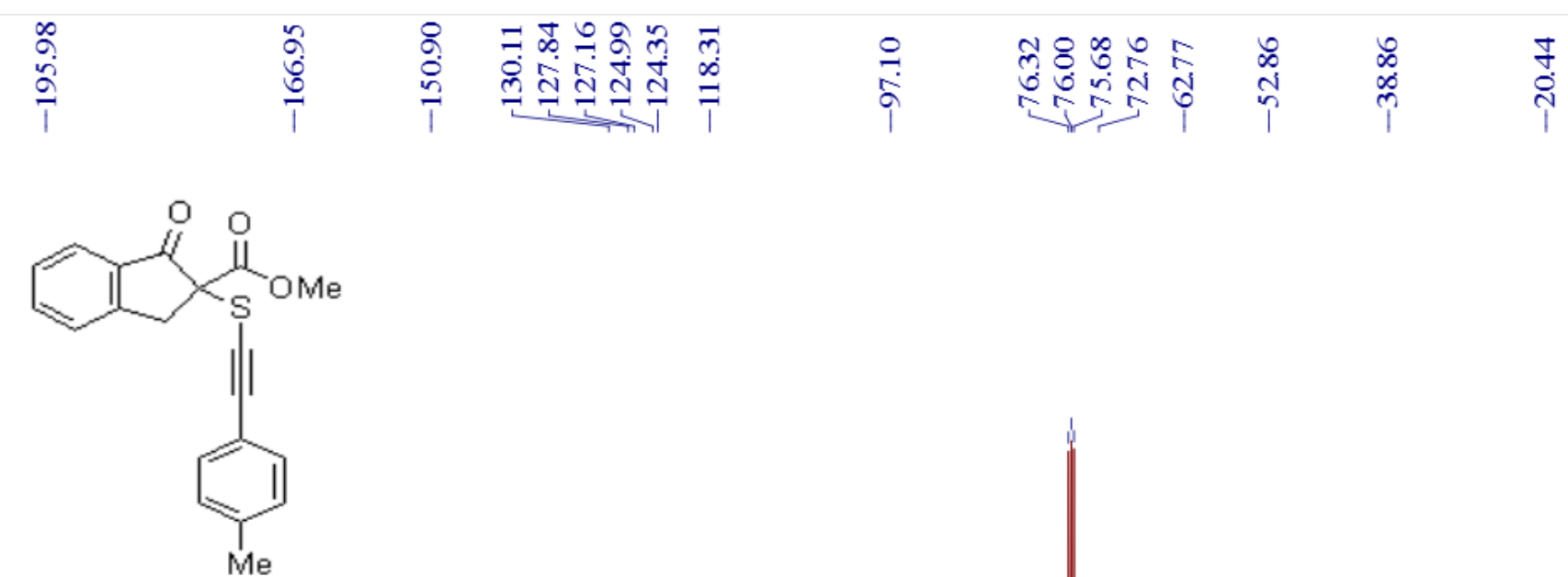

${ }^{13} \mathrm{C}$ NMR $100 \mathrm{MHz} \mathrm{CDCl}_{3}$

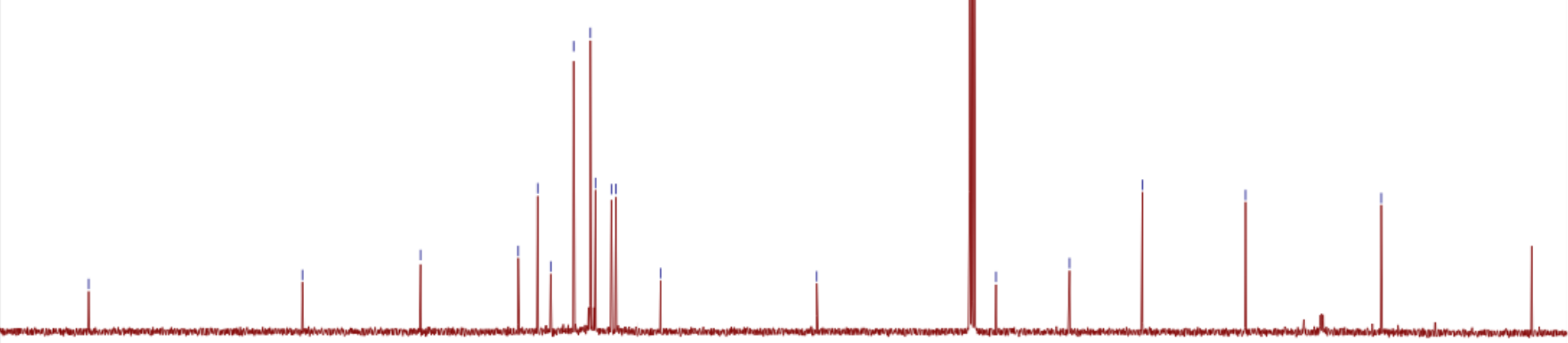

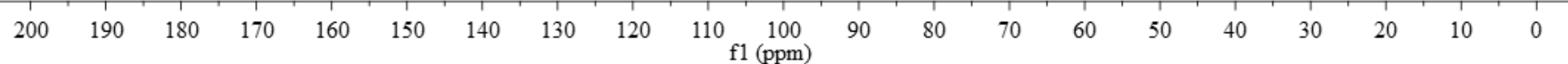




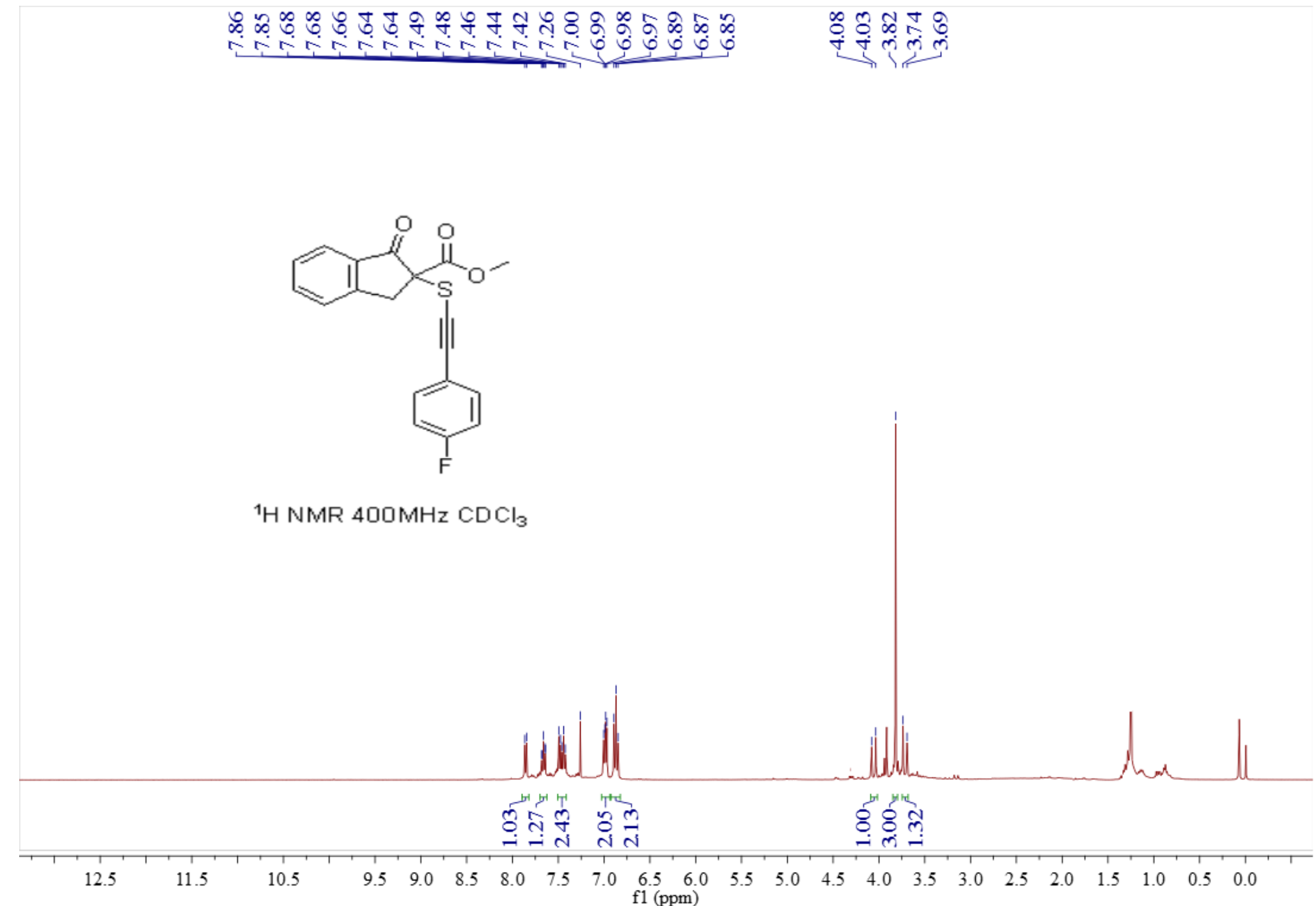



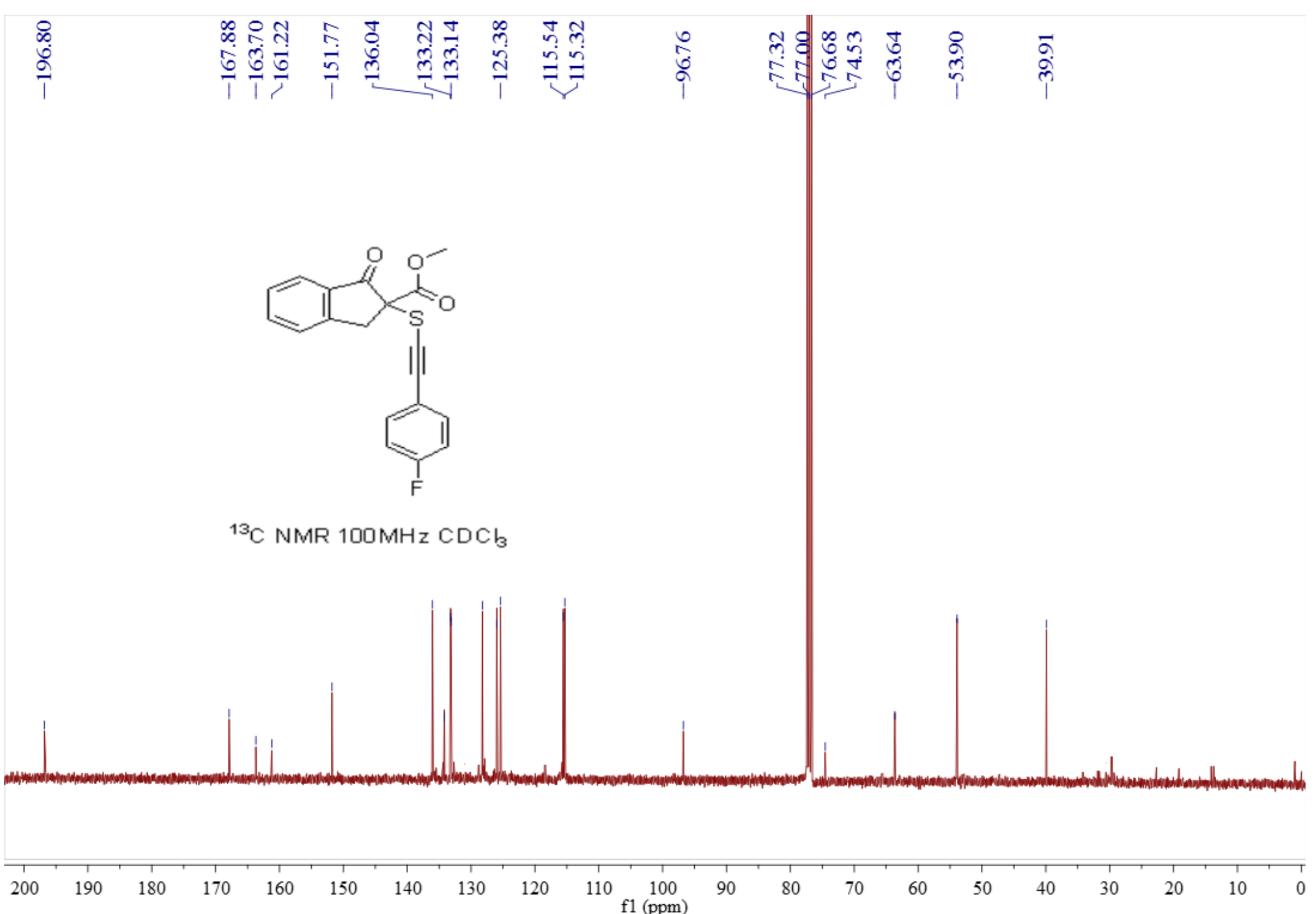


$$
\text { 些 }
$$



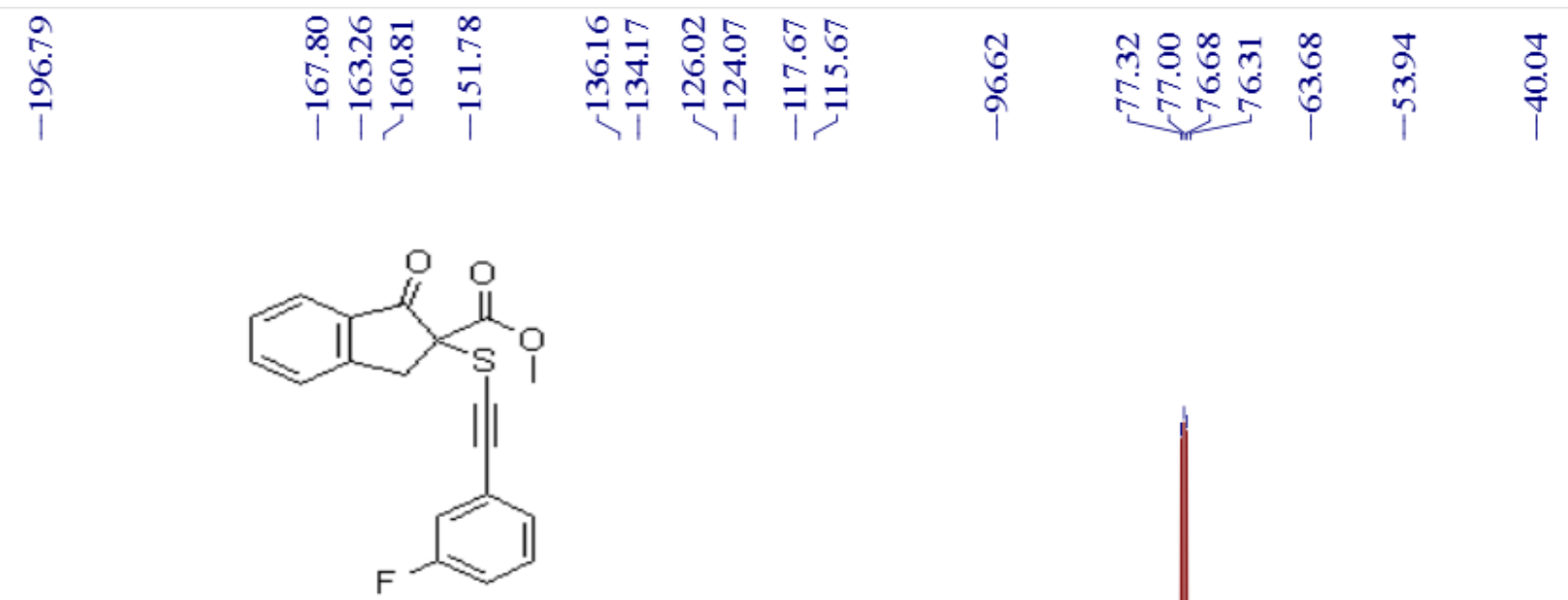

${ }^{13} \mathrm{C} \mathrm{NMR} 100 \mathrm{MHz} \mathrm{CDCl}_{3}$

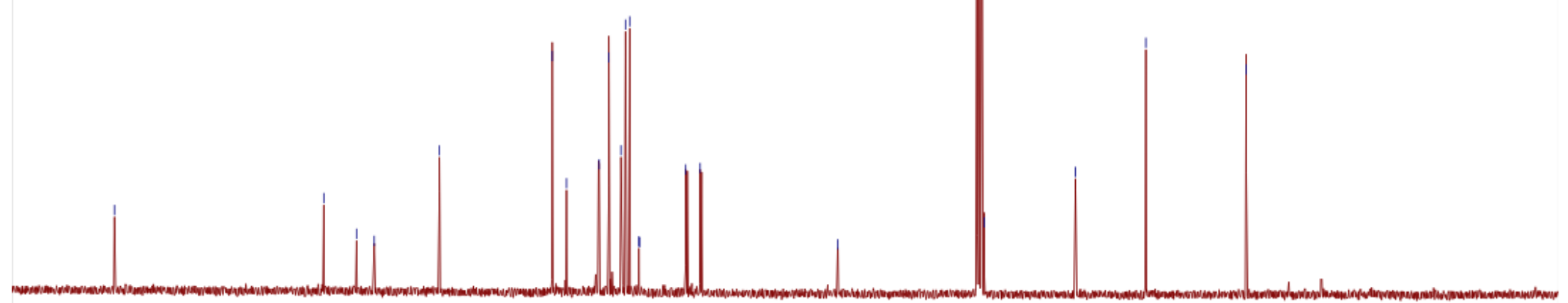

1

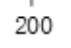

190

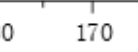

160

f1 $(\mathrm{ppm})$ 


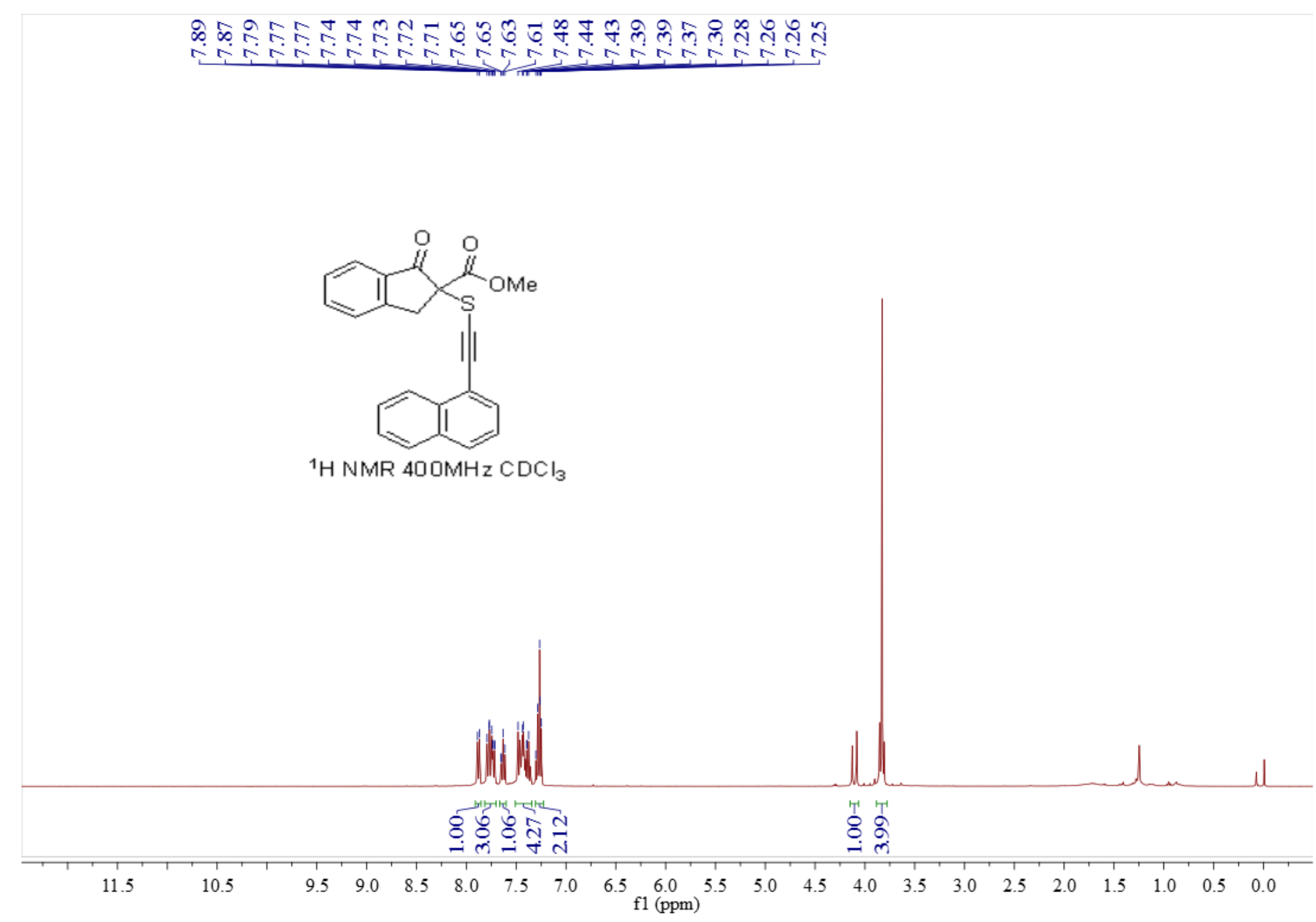




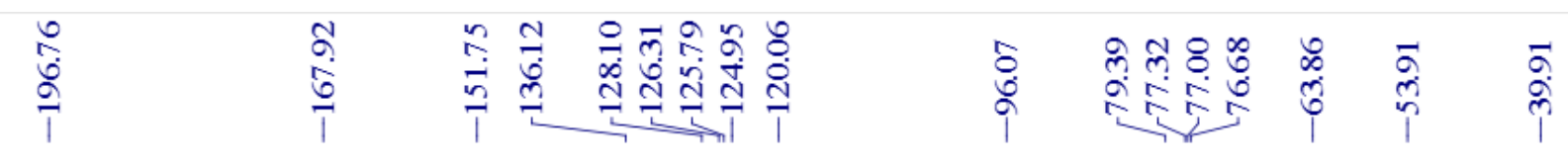

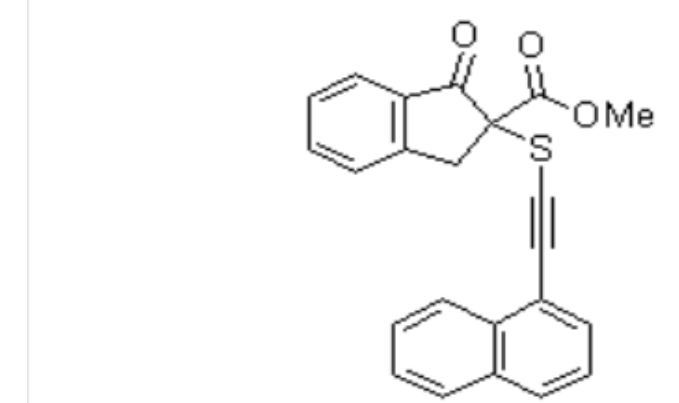

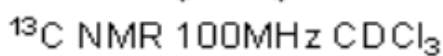
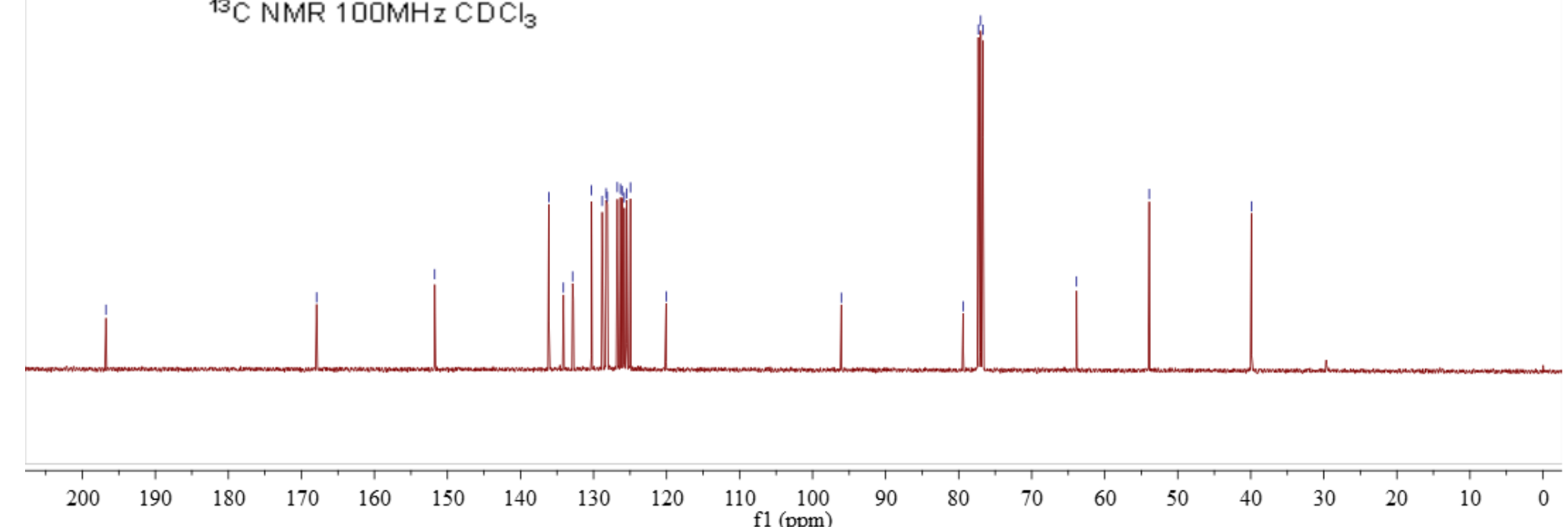


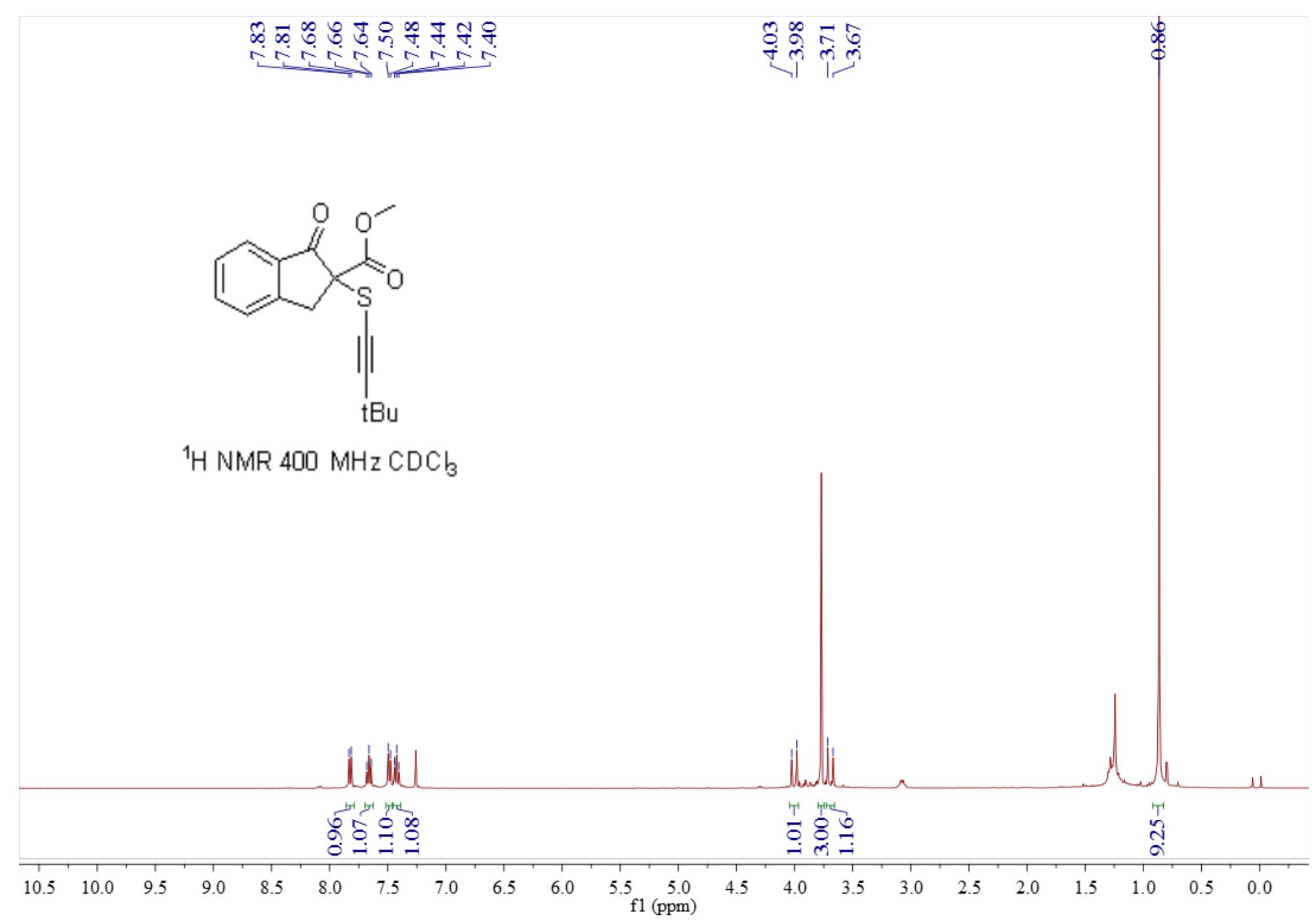




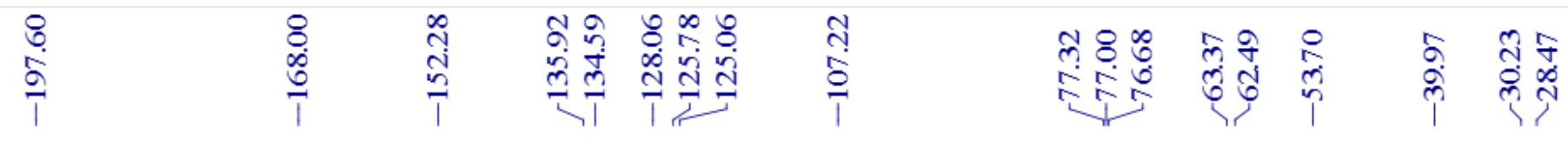

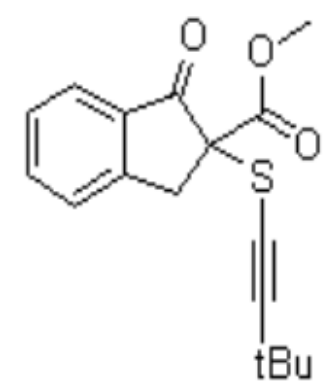

${ }^{13} \mathrm{C}$ NMR $100 \mathrm{MHz} \mathrm{CDCl}_{3}$
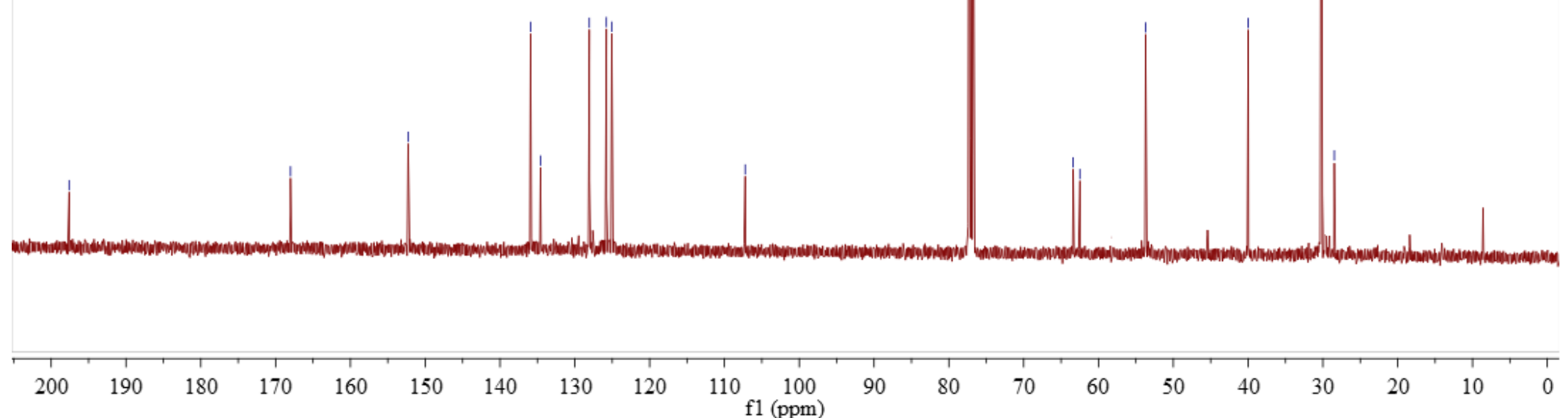


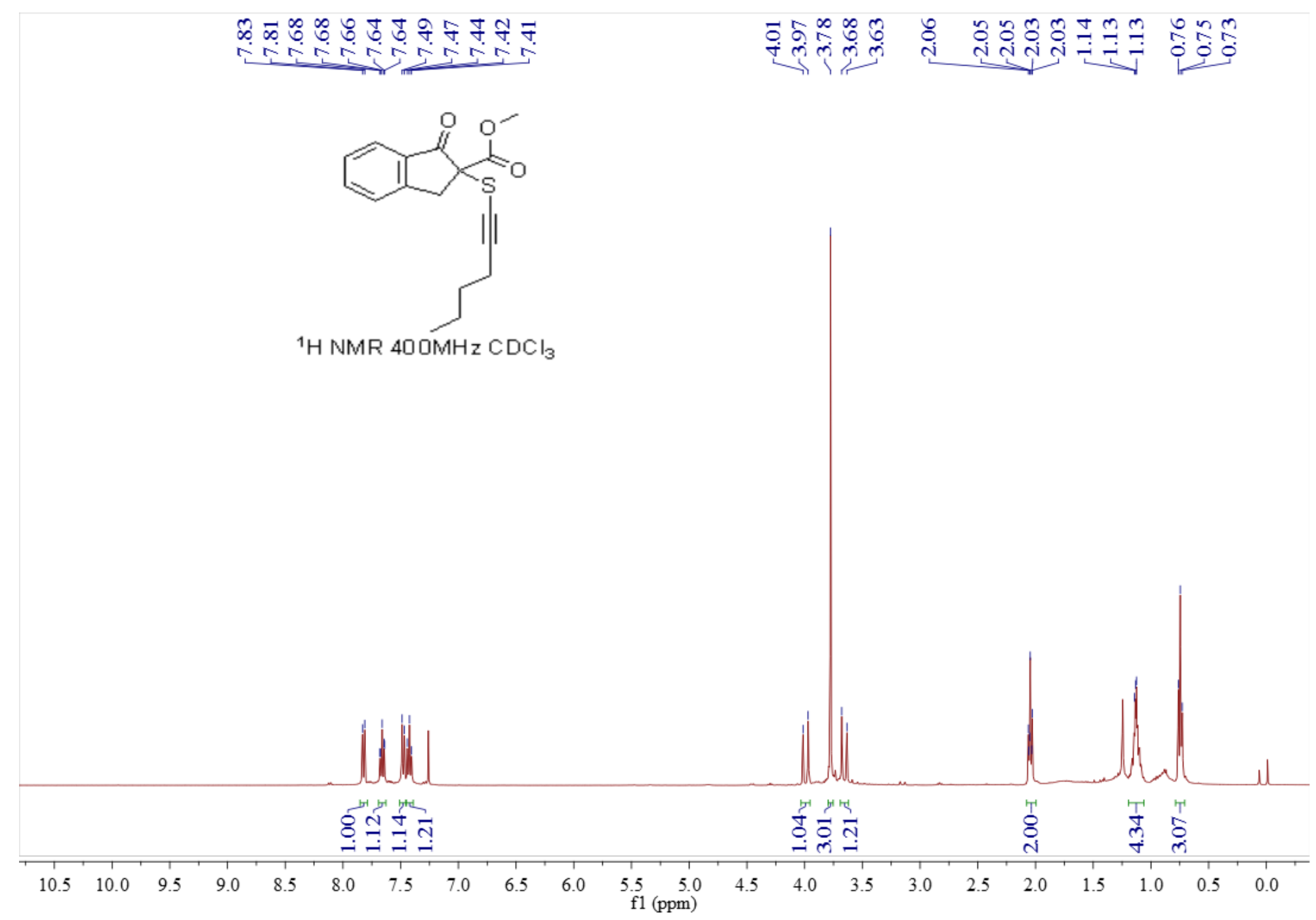




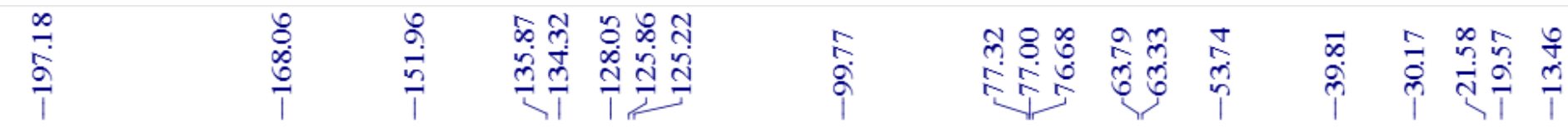

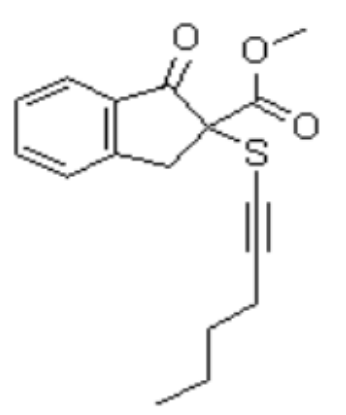

${ }^{13} \mathrm{C} \mathrm{NMR} 100 \mathrm{MHz} \mathrm{CDCl}_{3}$

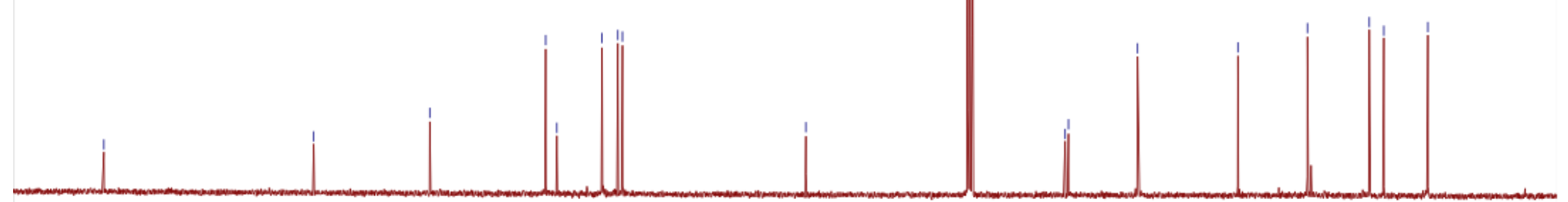

$\begin{array}{lllllllllll}200 & 190 & 180 & 170 & 160 & 150 & 140 & 130 & 120 & 110 & 100\end{array}$ 


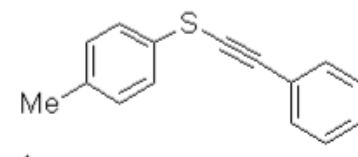

${ }^{1} \mathrm{HNMR} 400 \mathrm{MHz} \mathrm{CDCl}_{3}$

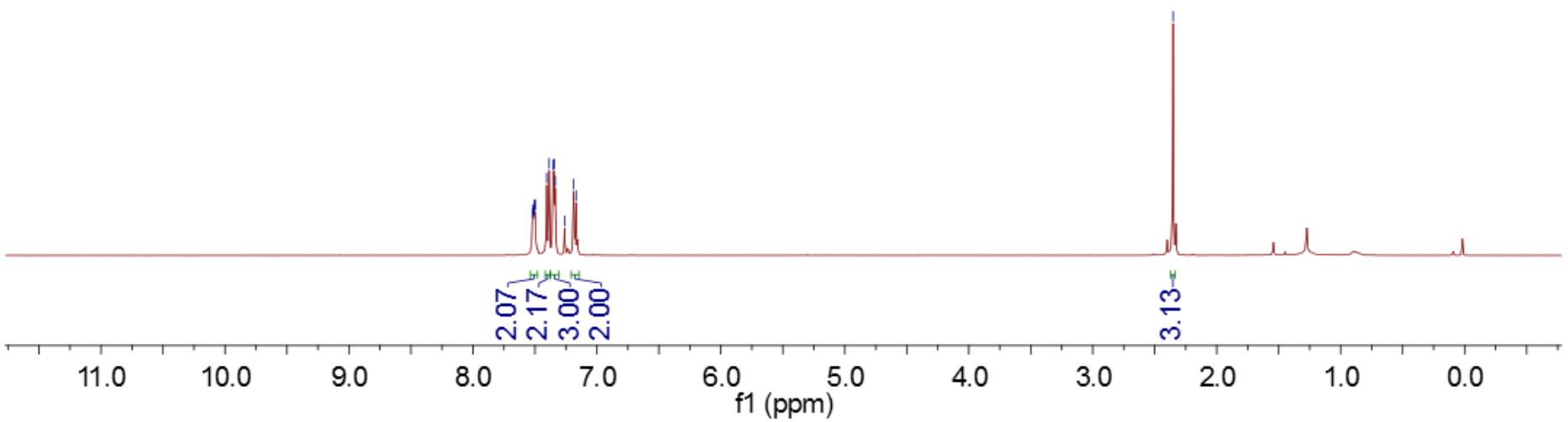




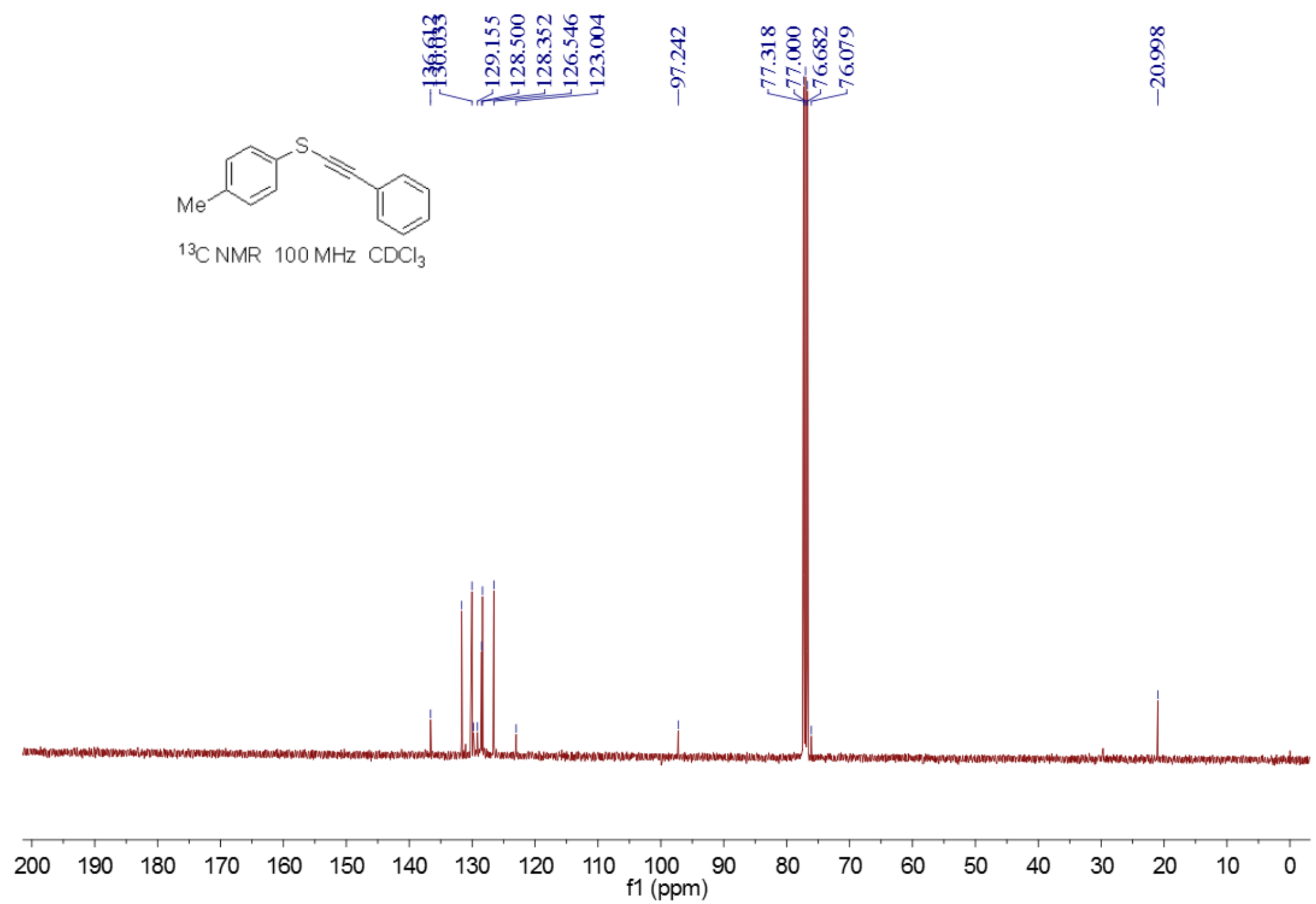




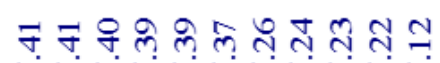

तर 25055

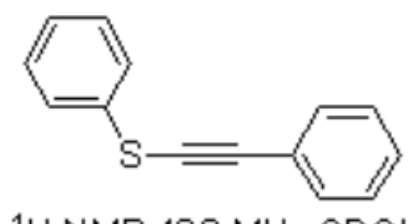

${ }^{1} \mathrm{HNMR} 400 \mathrm{MHz}^{\mathrm{CDCl}}$

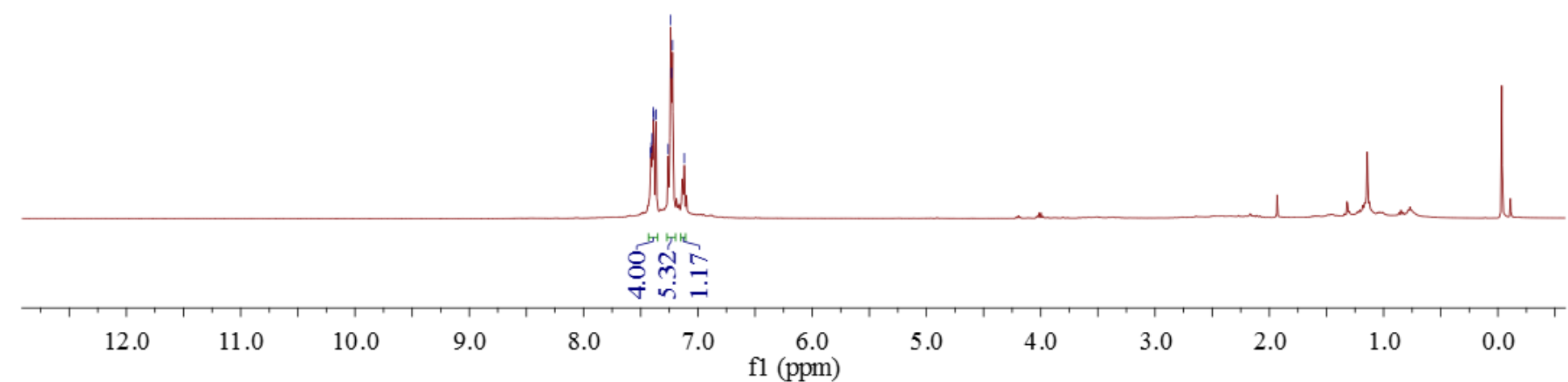




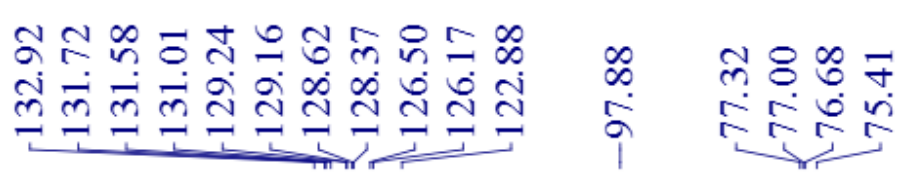

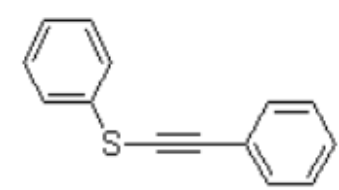

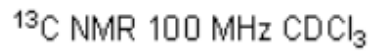
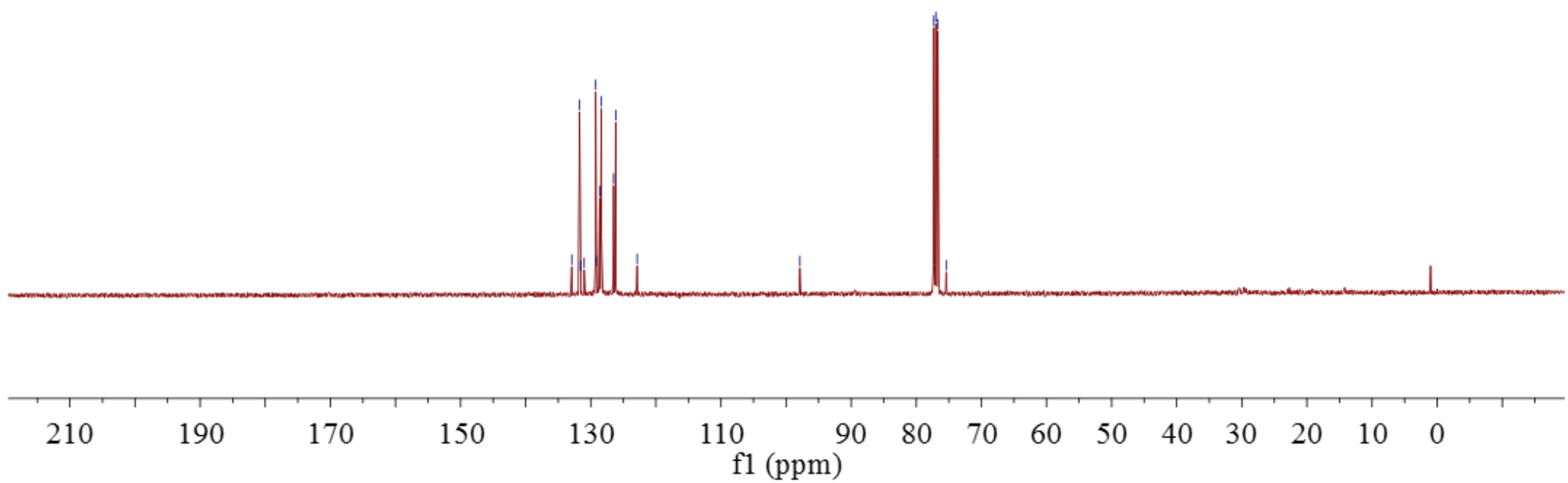


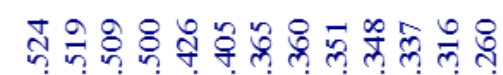

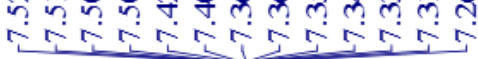

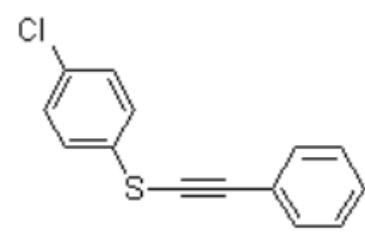

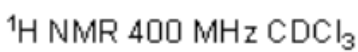

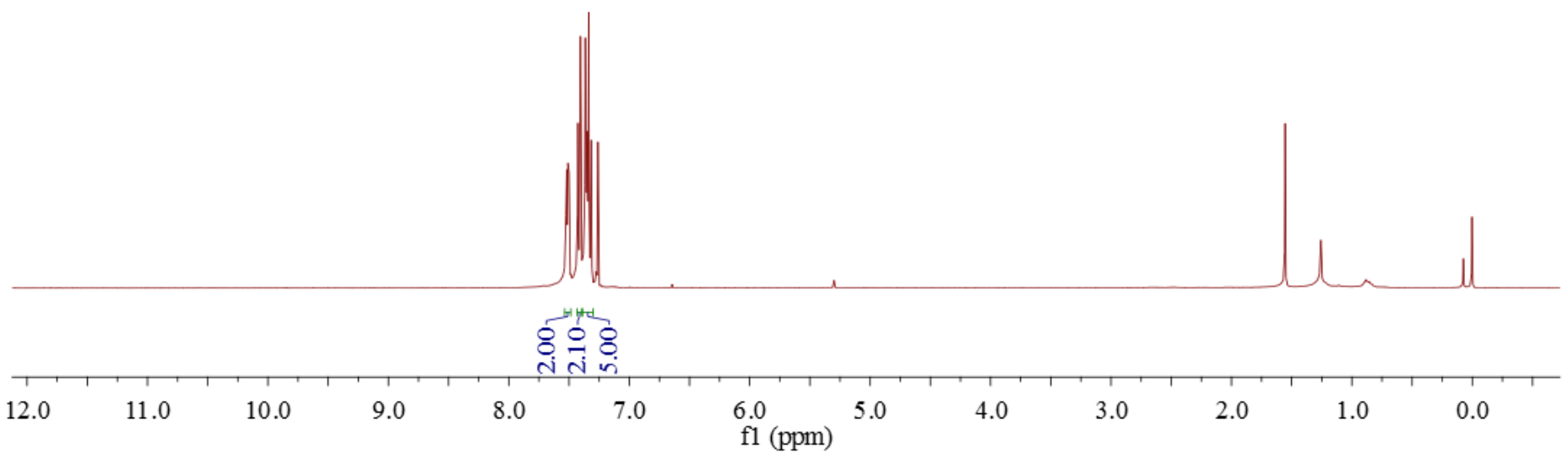




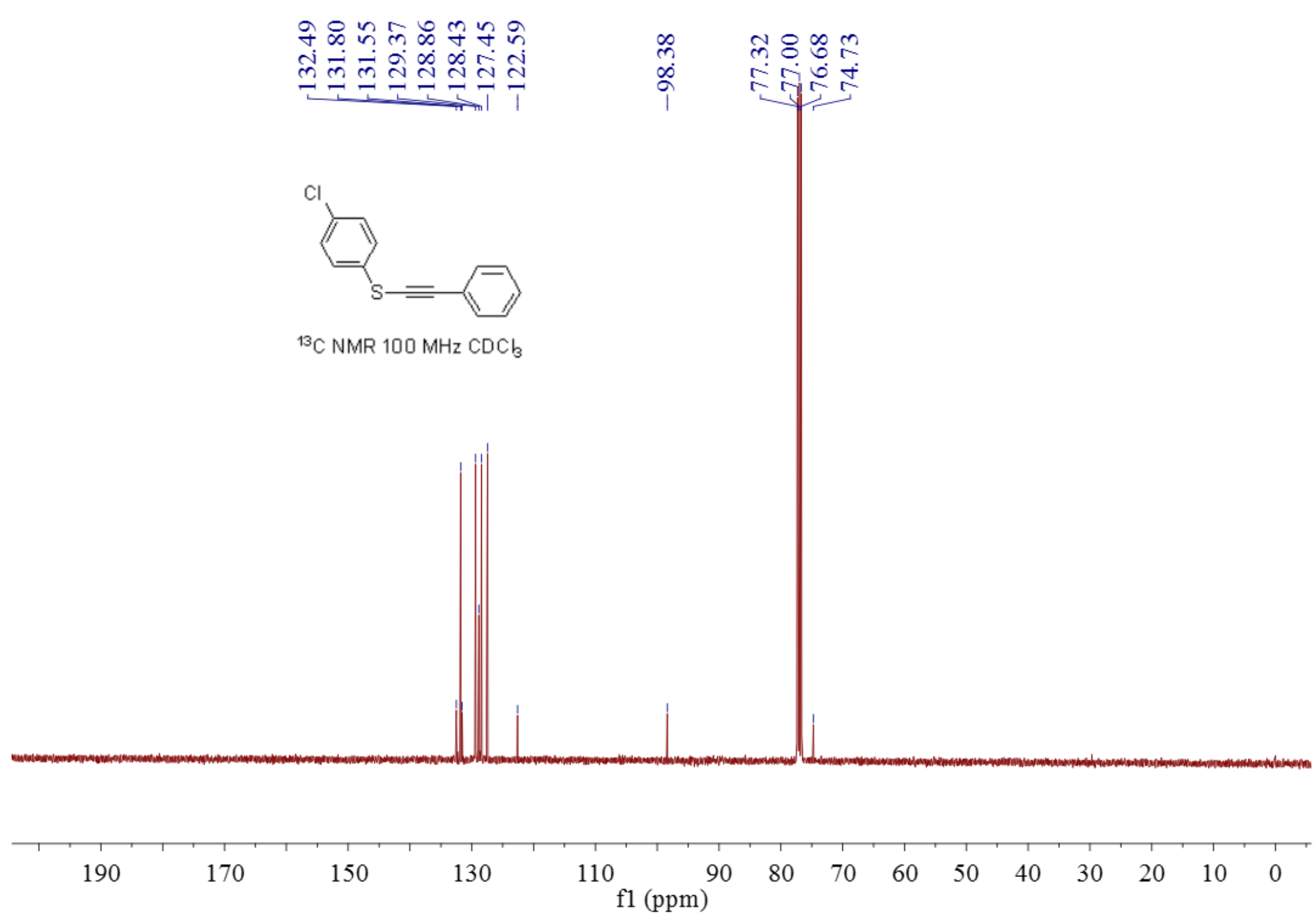




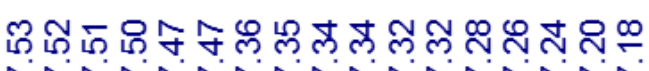

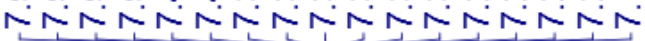

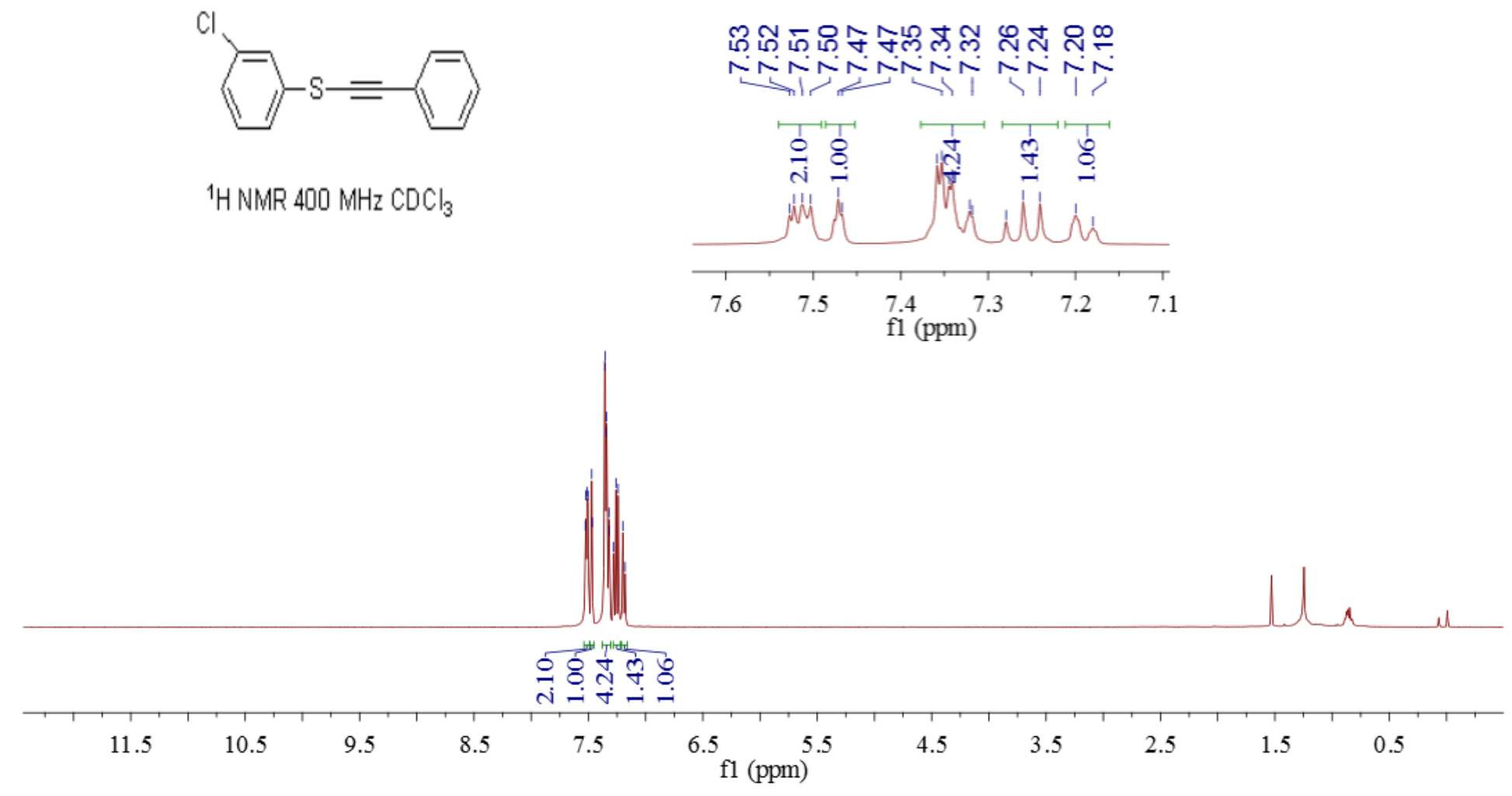



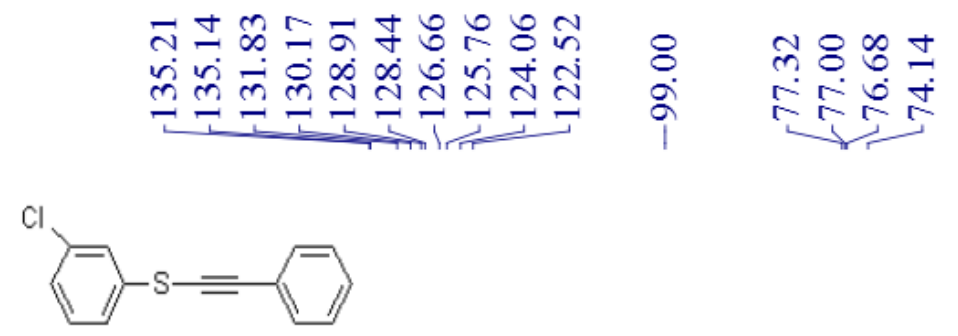

${ }^{13} \mathrm{C}$ NMR $400 \mathrm{MHz} \mathrm{CDCl}$
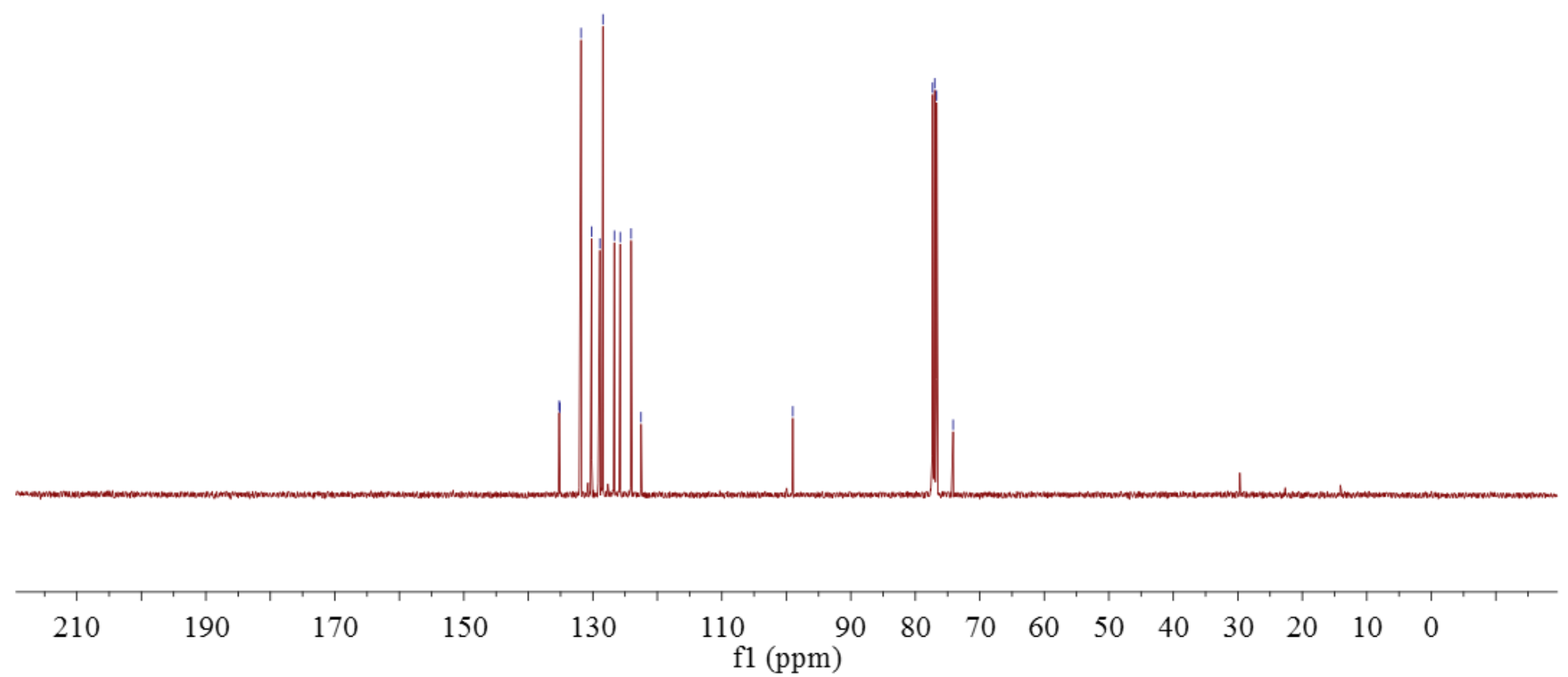


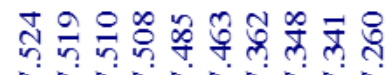

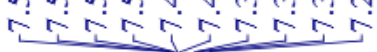

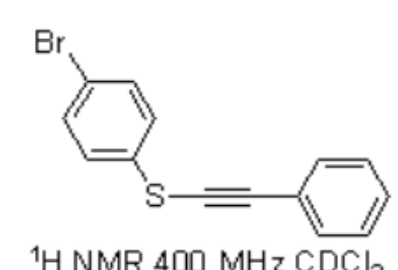

${ }^{1} \mathrm{H} \mathrm{NMR} 400 \mathrm{MHz}_{\mathrm{CDCl}_{3}}$

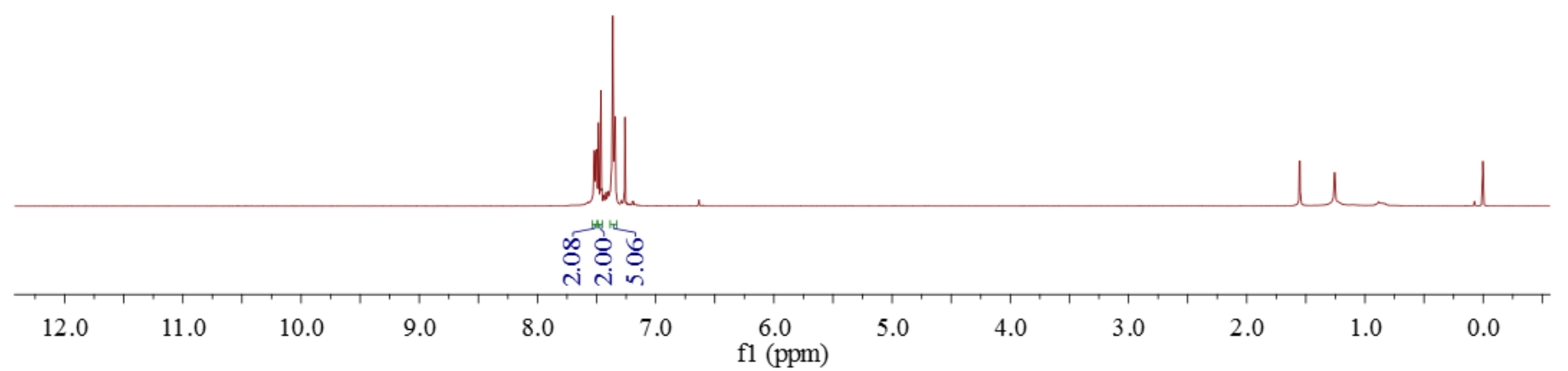


수 $\infty$

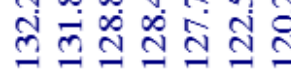

$n$
$n$
$\infty$

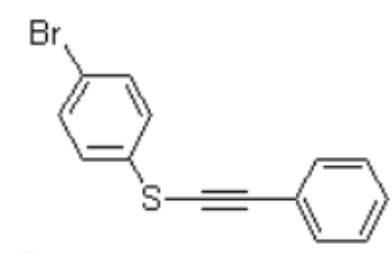

${ }^{13} \mathrm{CNMR} 100 \mathrm{MHz}^{\mathrm{CDCl}} 3$

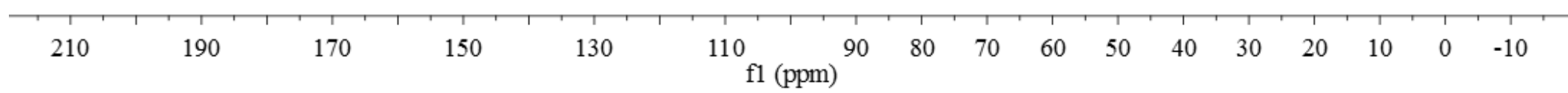




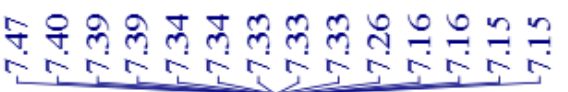

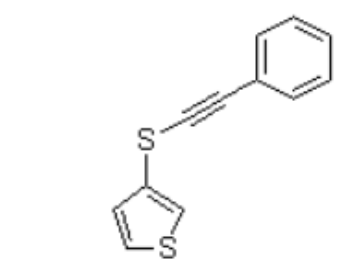

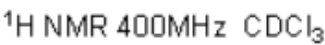

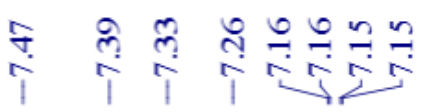
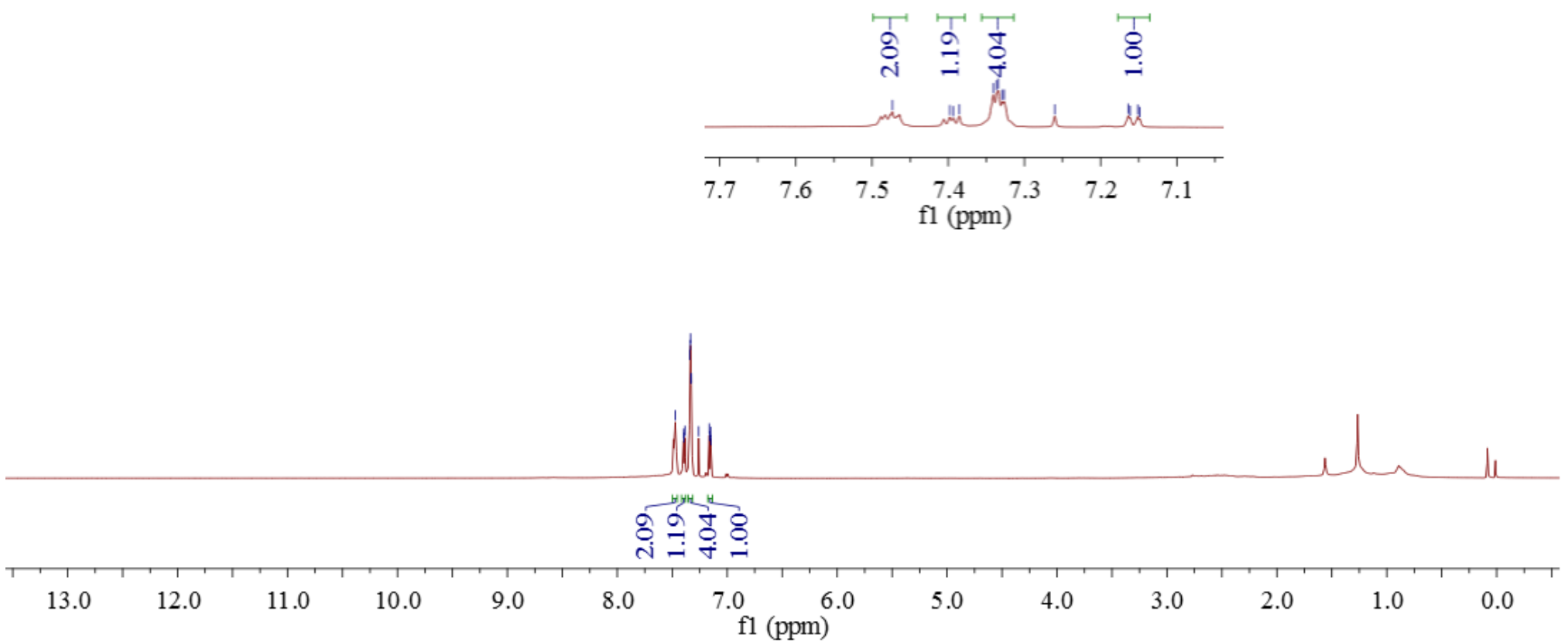


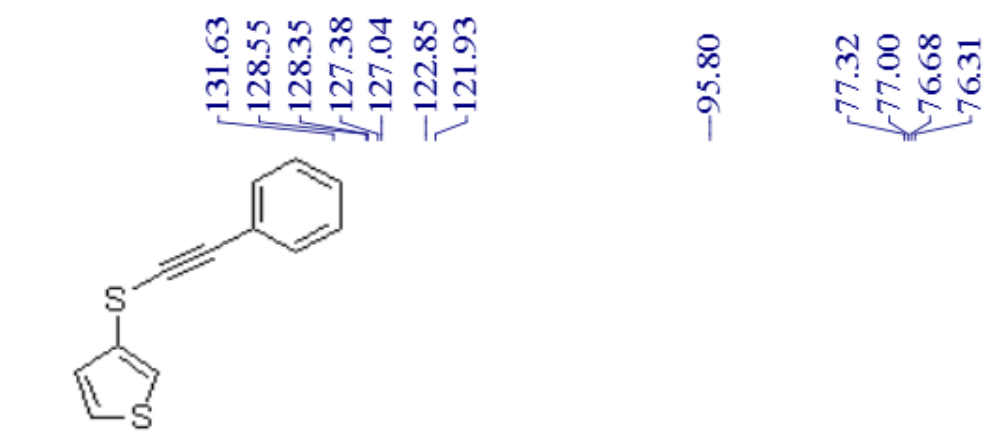

${ }^{13} \mathrm{C} \mathrm{NMR}^{100 M H z} \mathrm{CDCl}_{3}$

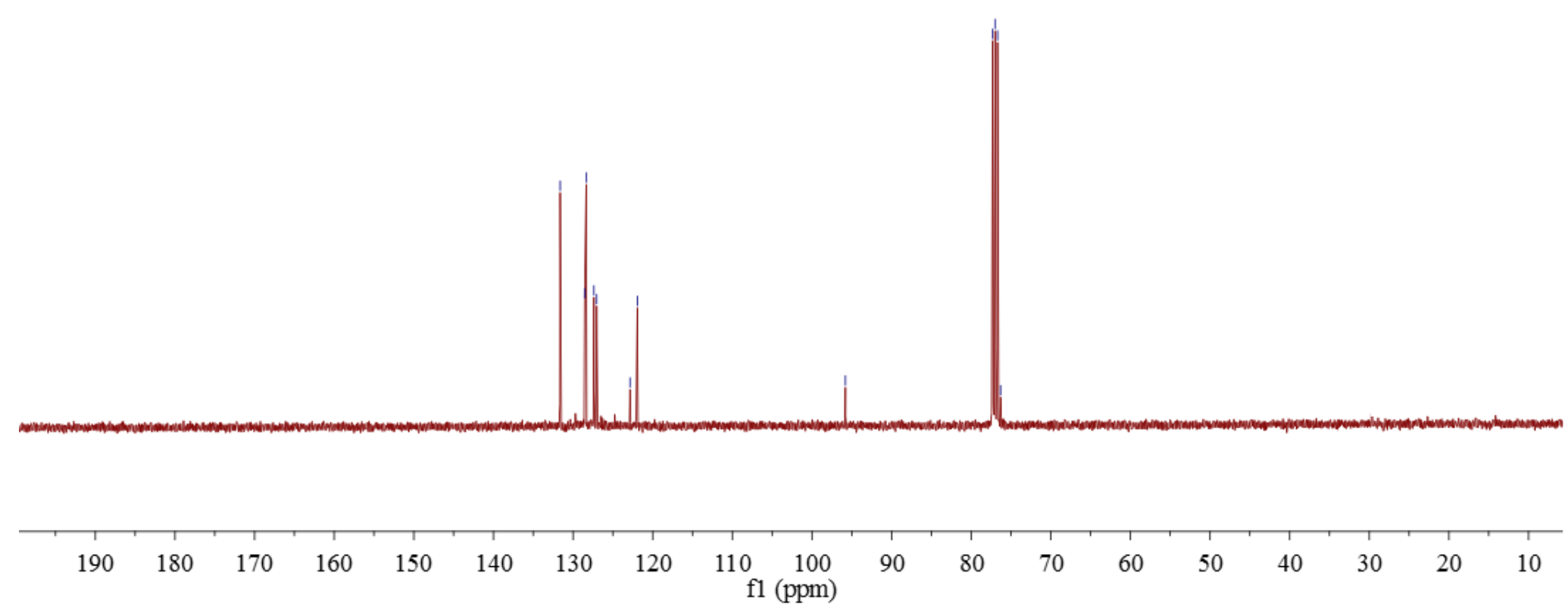




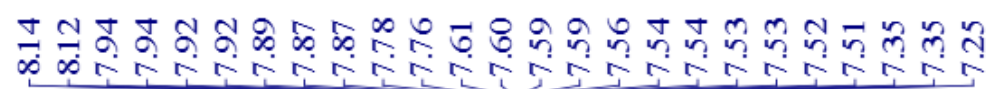

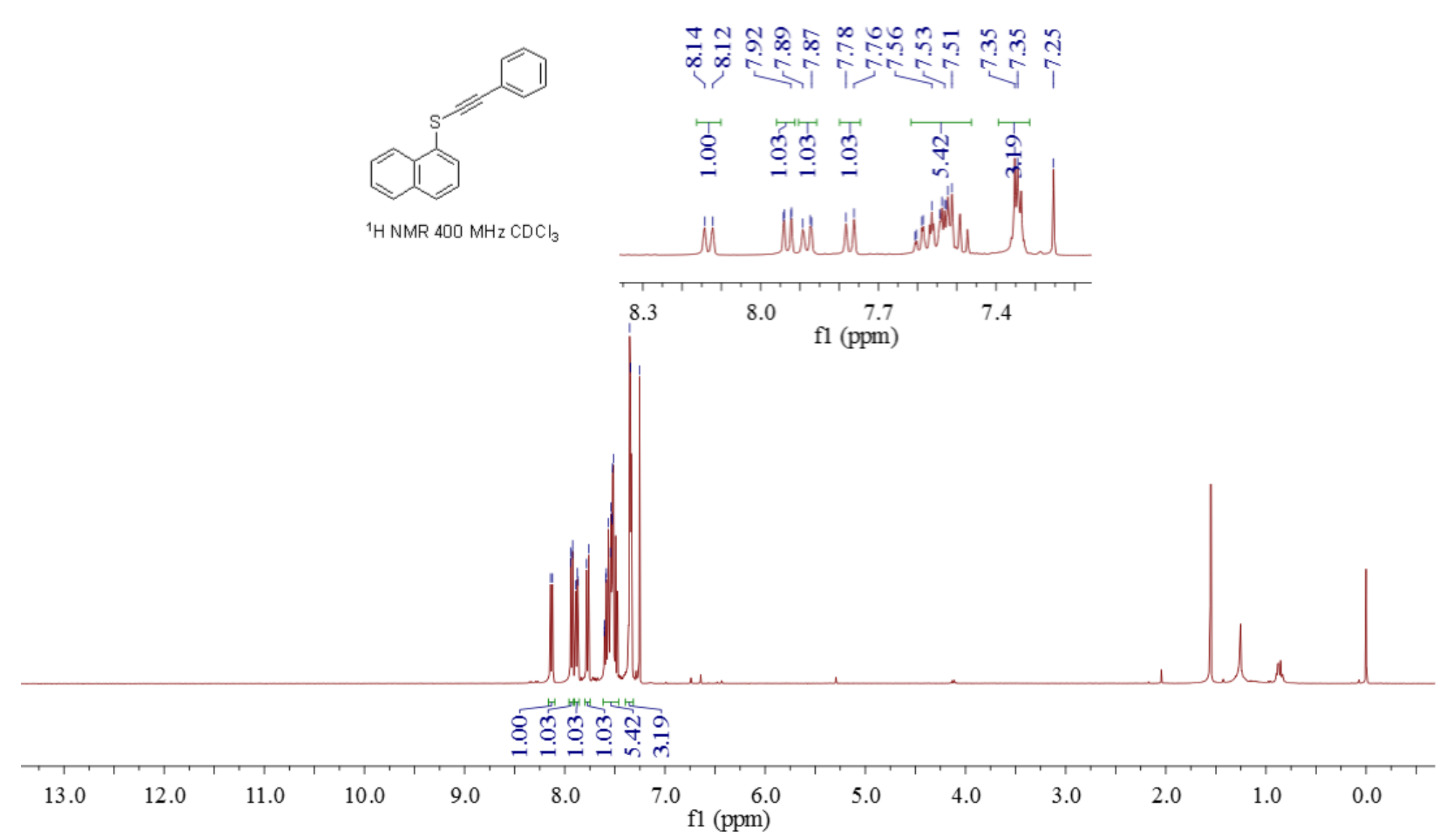




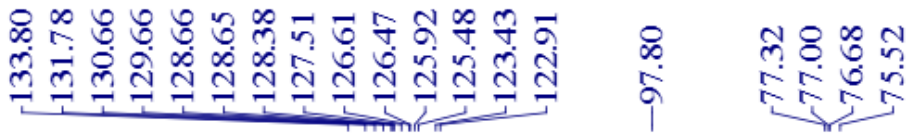

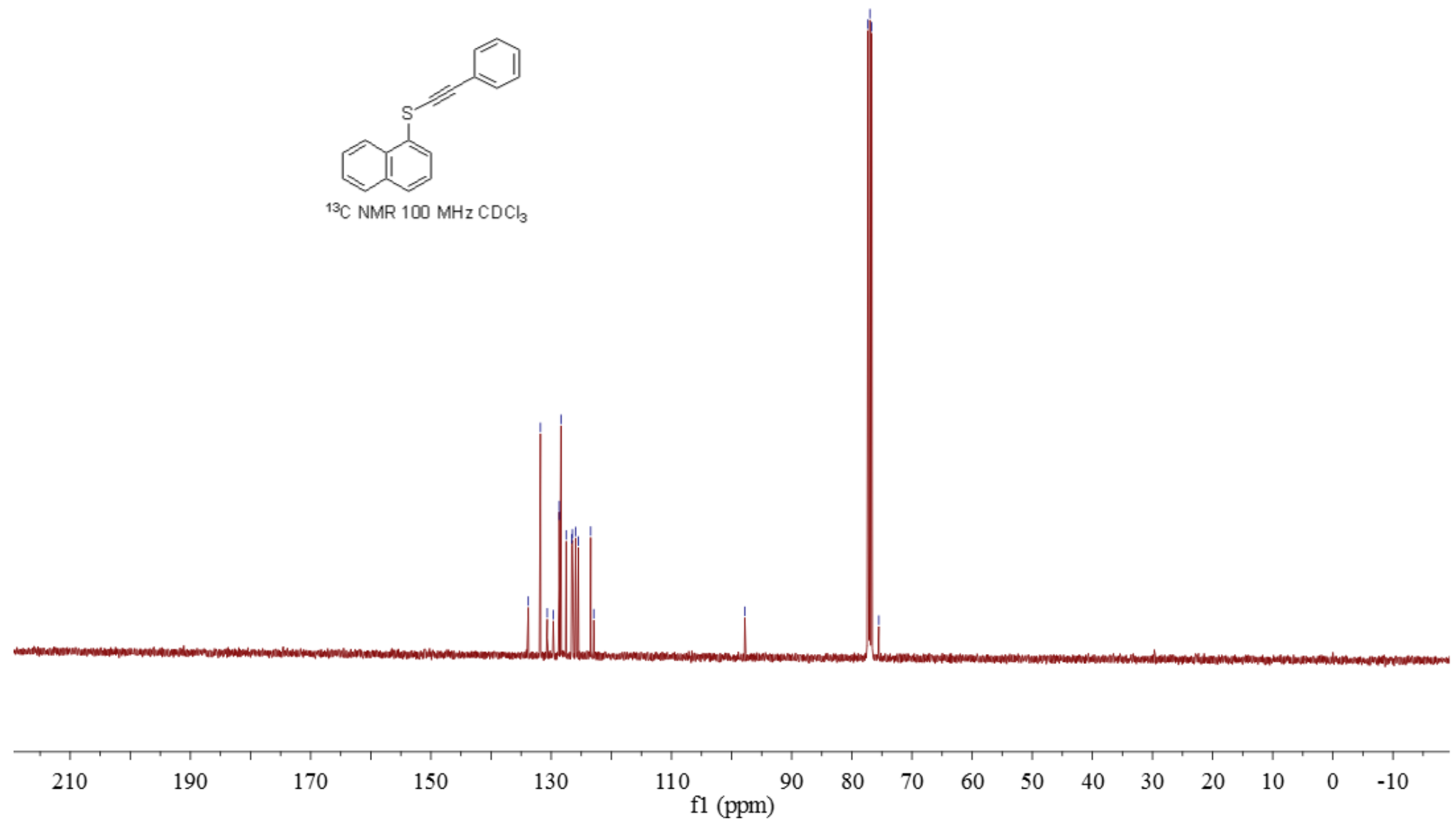



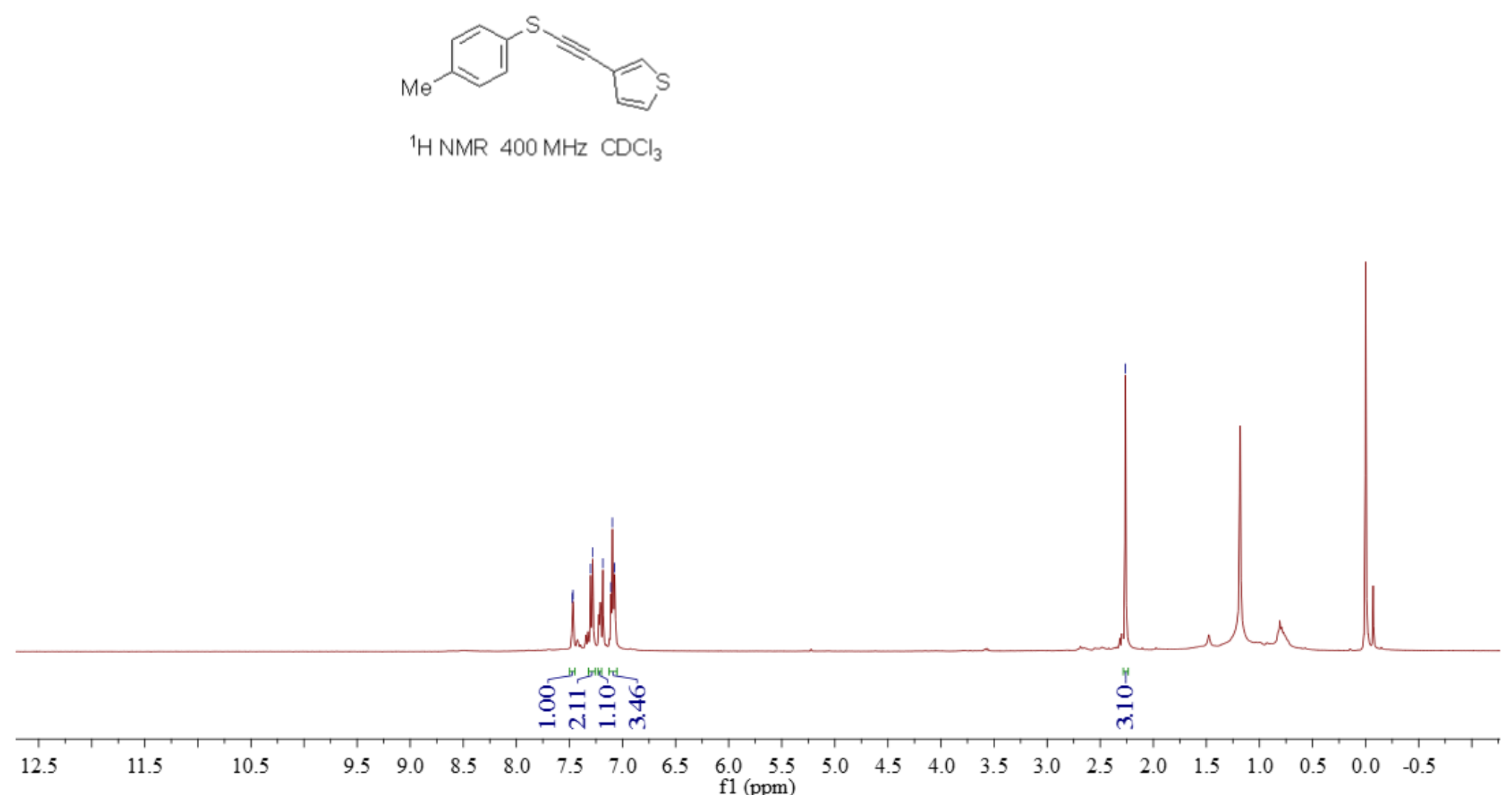

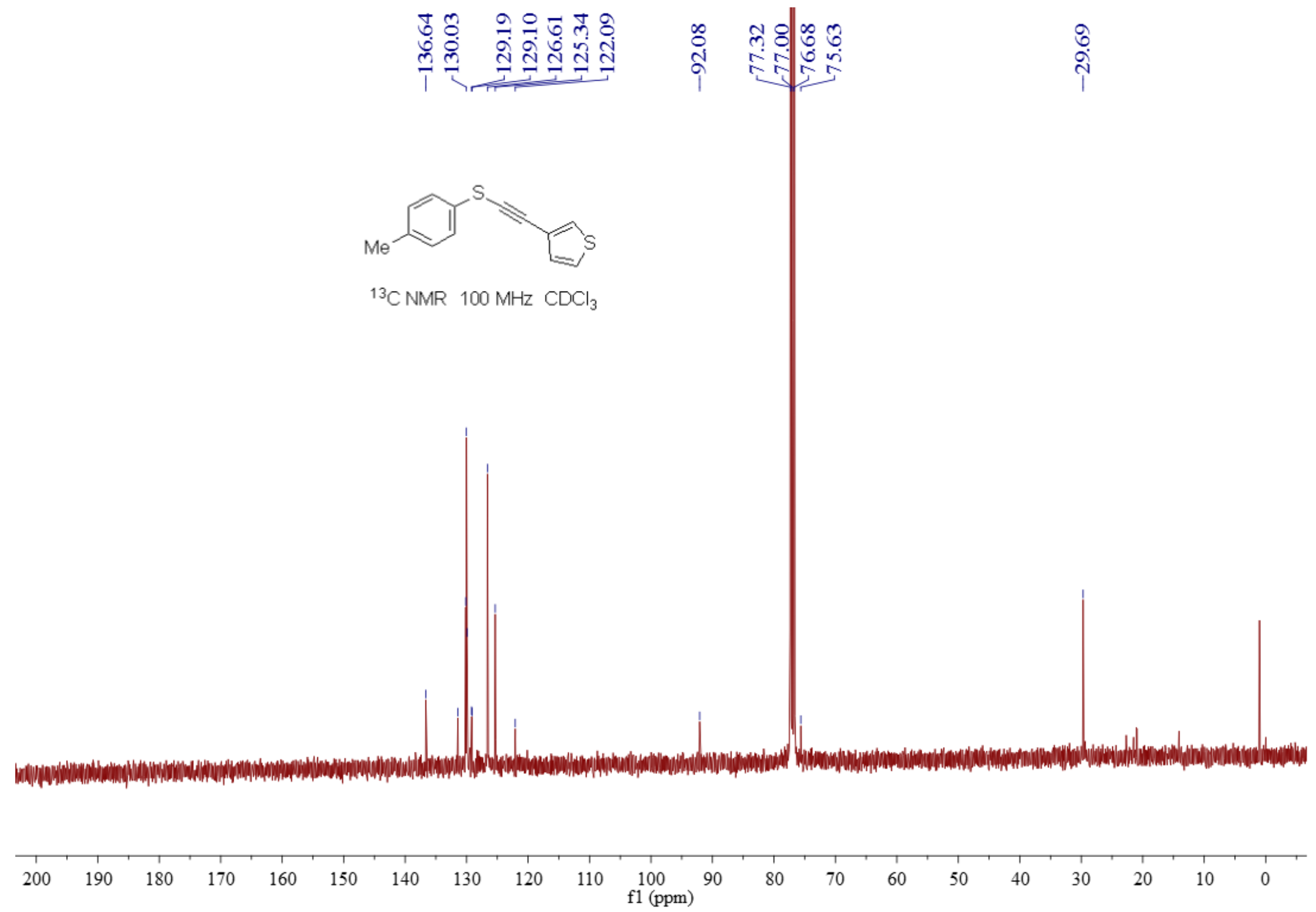


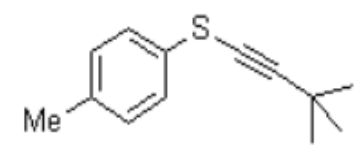

${ }^{1} \mathrm{H} \mathrm{NMR} 400 \mathrm{MHz} \mathrm{CDCl}$

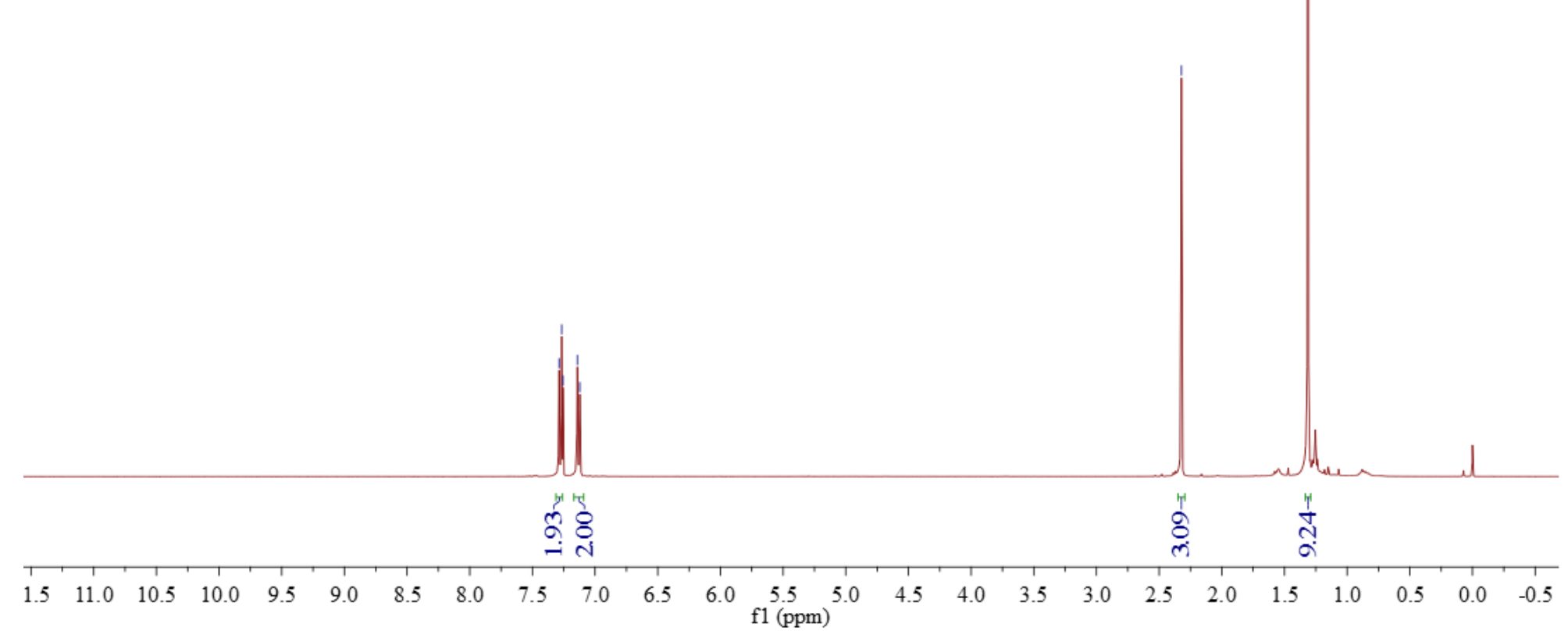




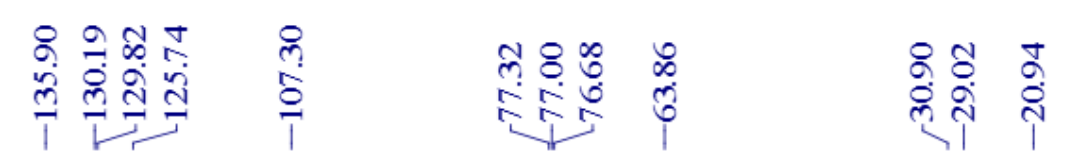

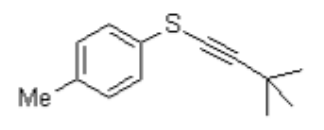

${ }^{13} \mathrm{CNMR} 100 \mathrm{MHz} \mathrm{CDCl}_{3}$

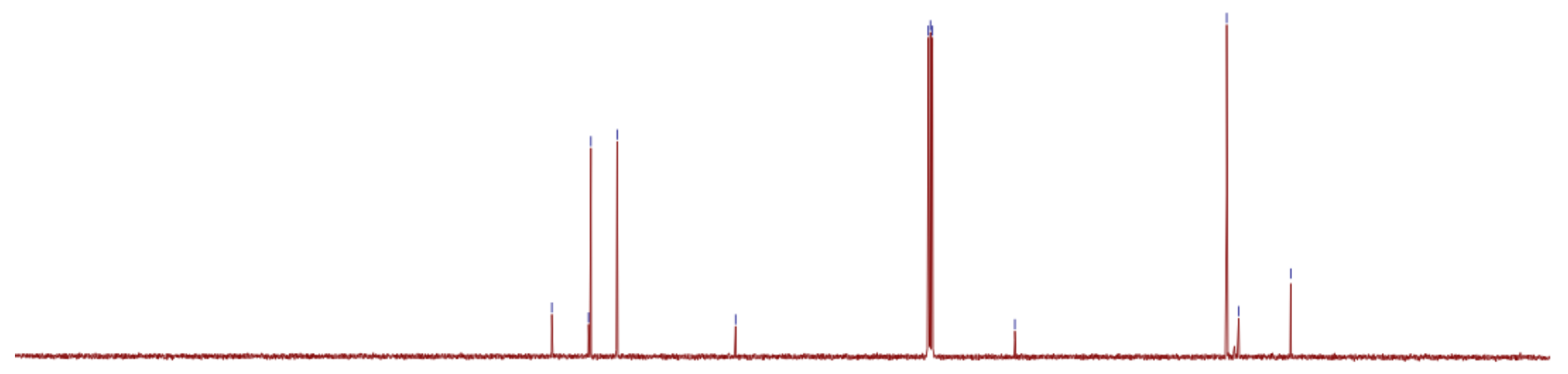

$\begin{array}{llllllllllll}210 & 200 & 190 & 180 & 170 & 160 & 150 & 140 & 130 & 120 & 110 & 100\end{array}$ f1 (ppm) 


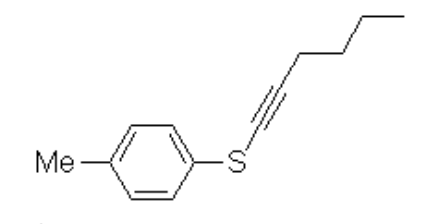

${ }^{1} \mathrm{H}$ NMR $400 \mathrm{MHz} \mathrm{CDCl}_{3}$

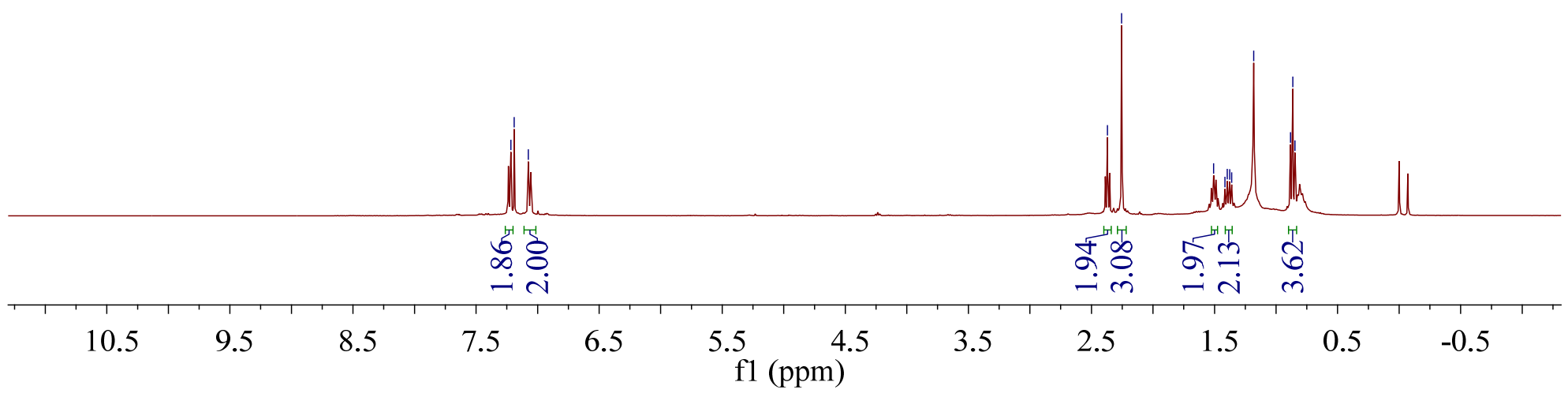




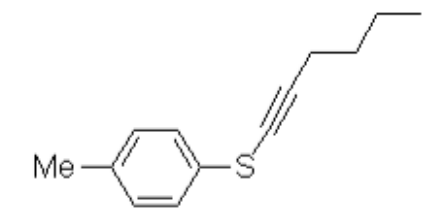

${ }^{13} \mathrm{CNMR} 100 \mathrm{MHz} \mathrm{CDCl}_{3}$

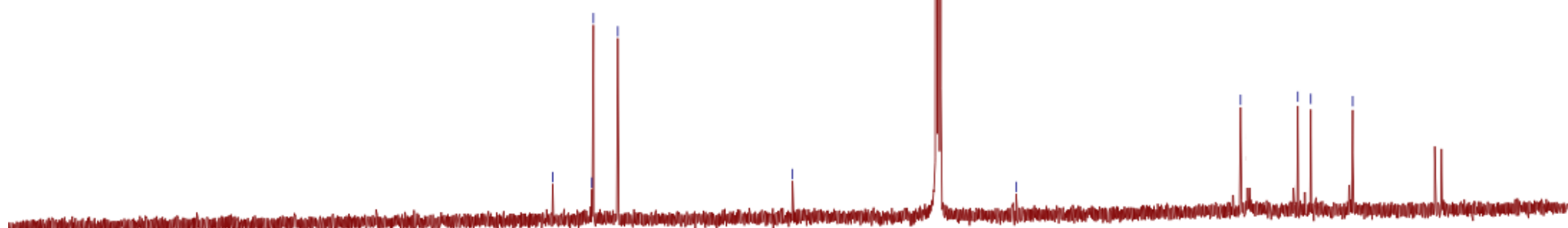

$\begin{array}{lllllllllllllllllllllllll}210 & 200 & 190 & 180 & 170 & 160 & 150 & 140 & 130 & 120 & 110 & 100 & 90 & 80 & 70 & 60 & 50 & 40 & 30 & 20 & 10 & 0 & -10 & \end{array}$ f1 (ppm) 

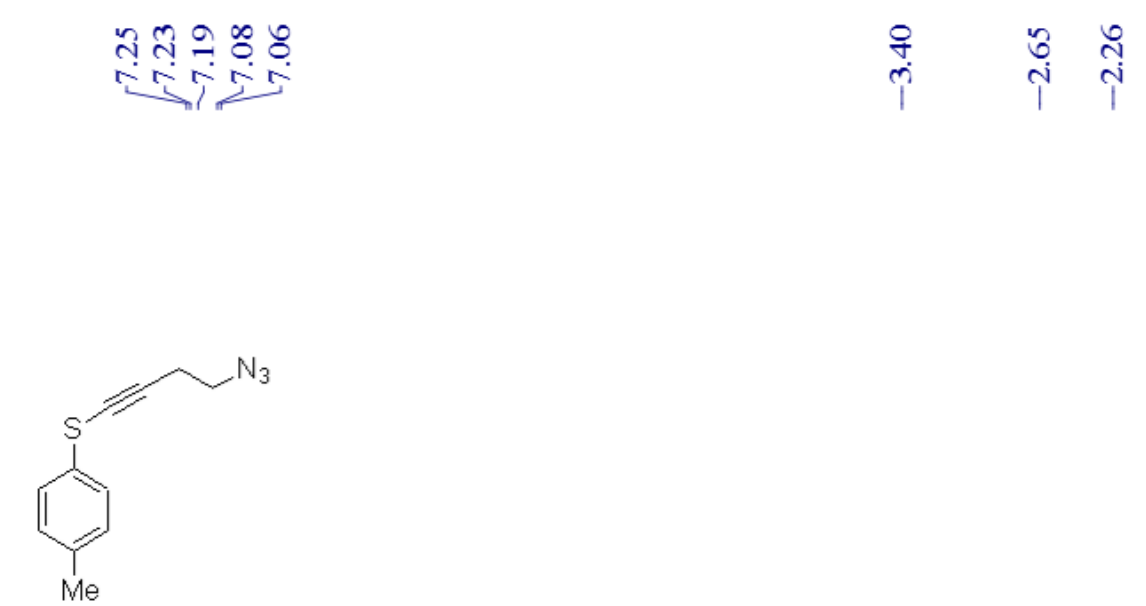

${ }^{1} \mathrm{HNMR} 400 \mathrm{MHz} \mathrm{CDCl}_{3}$

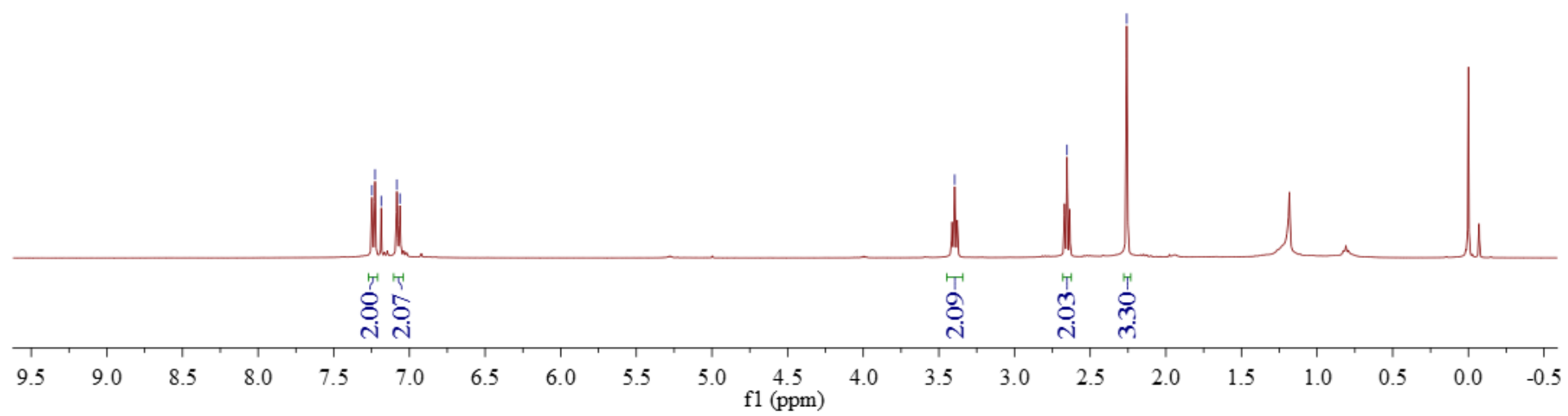




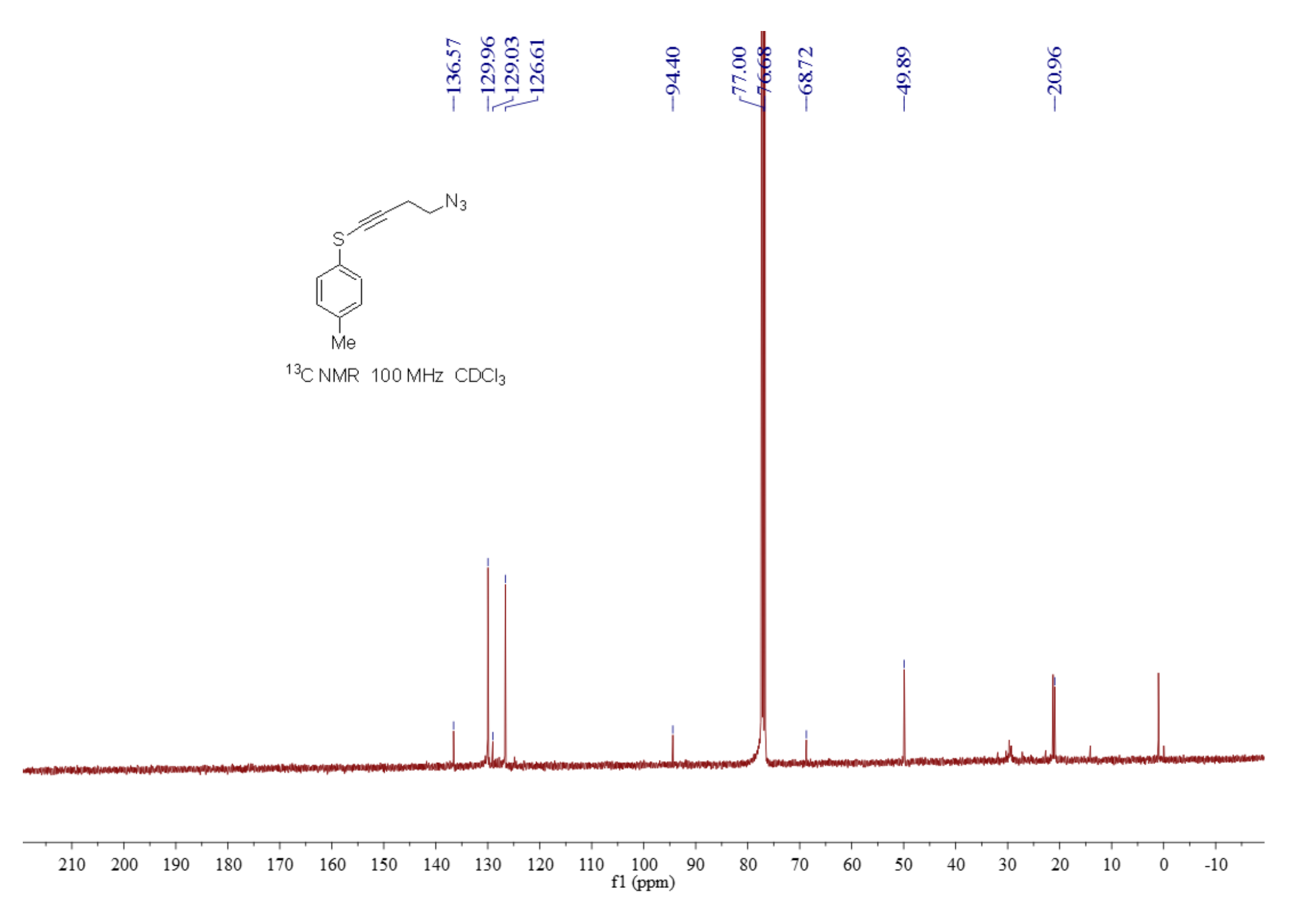



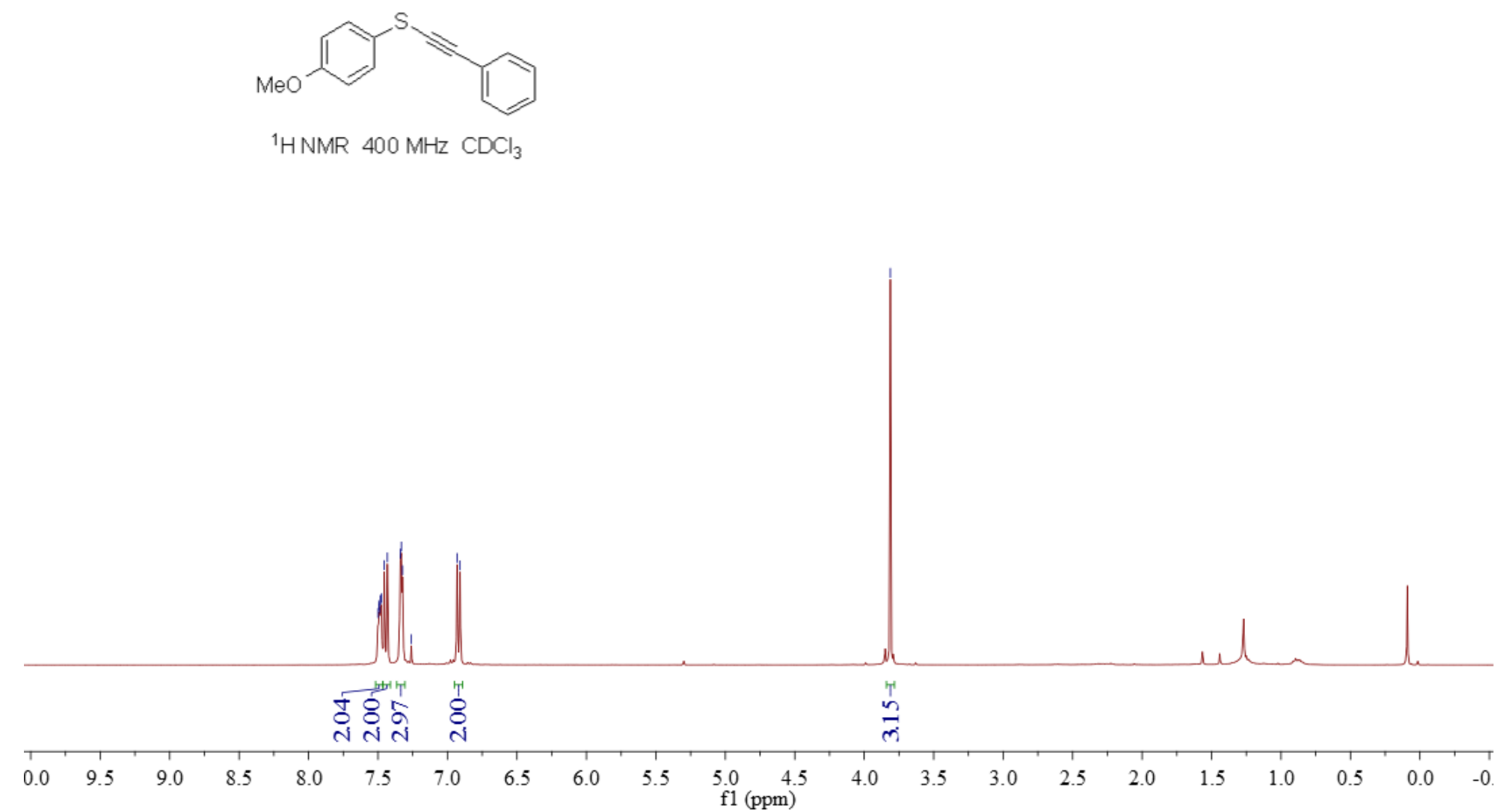


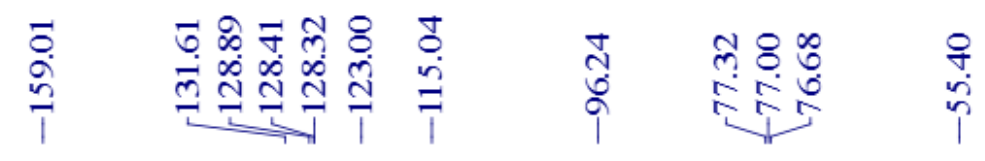
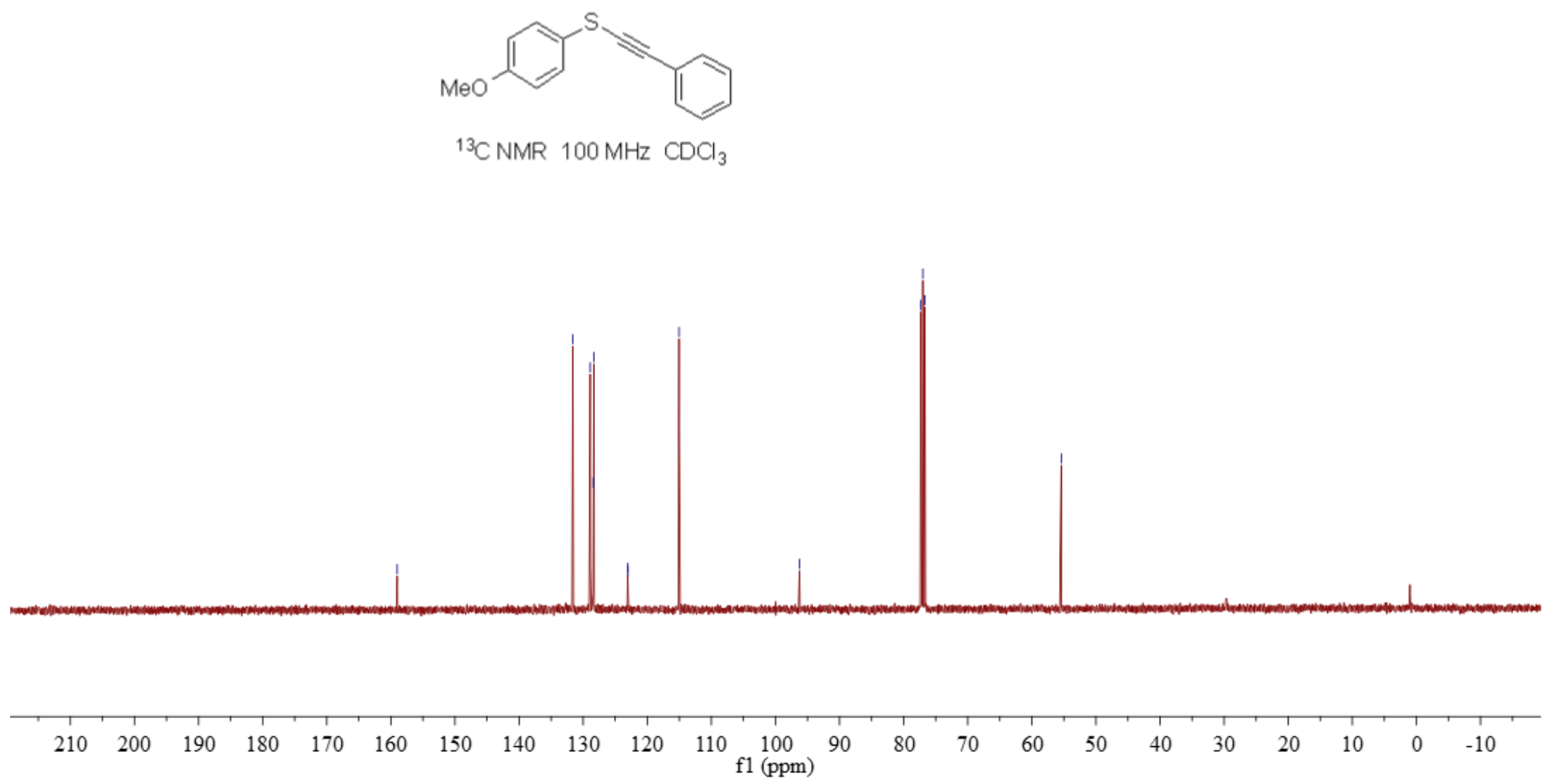


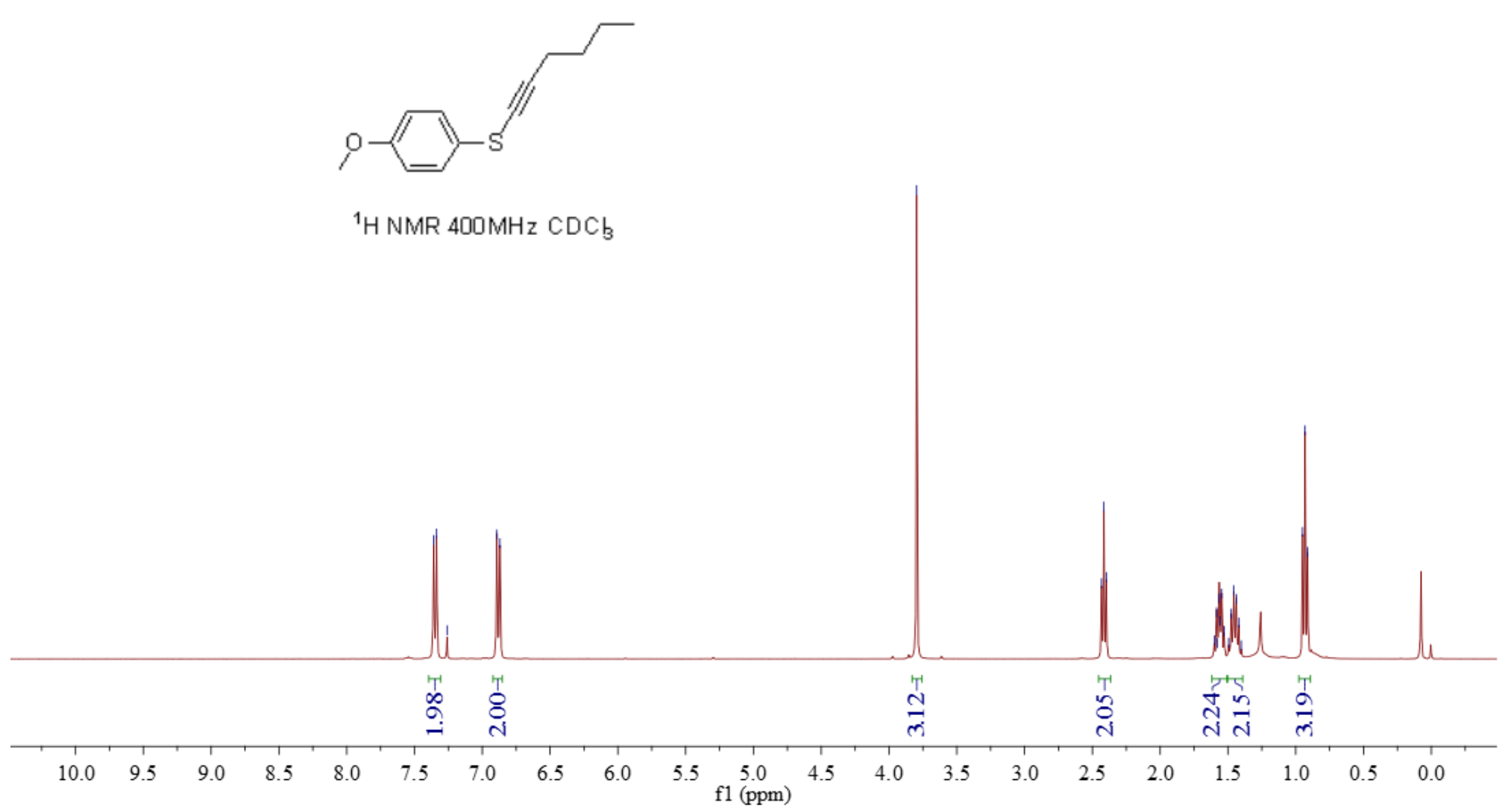




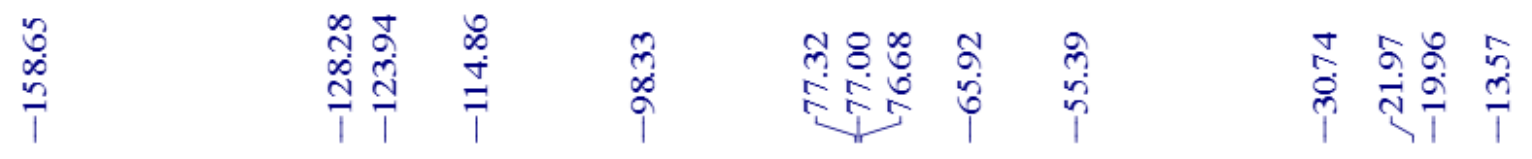

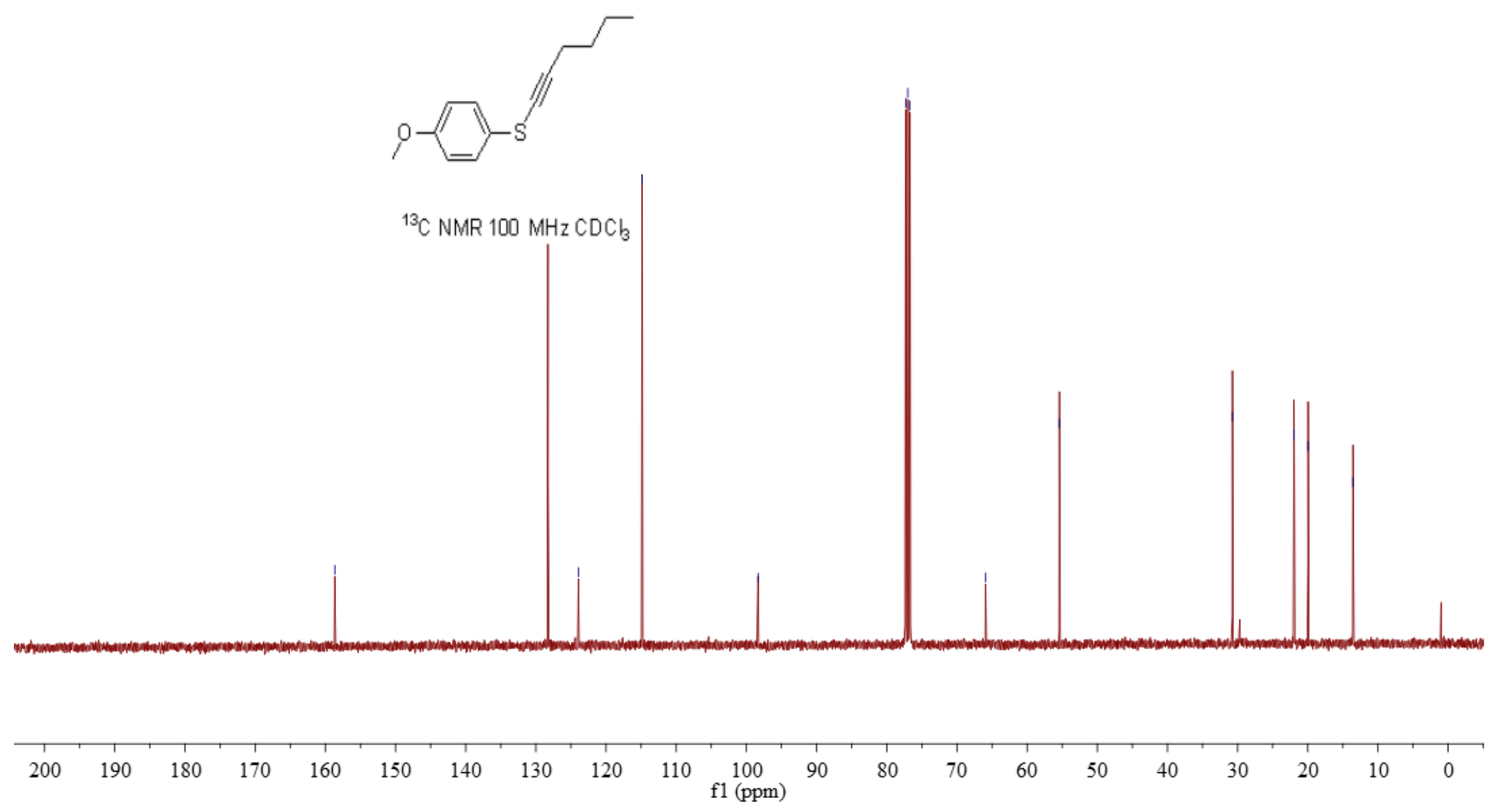




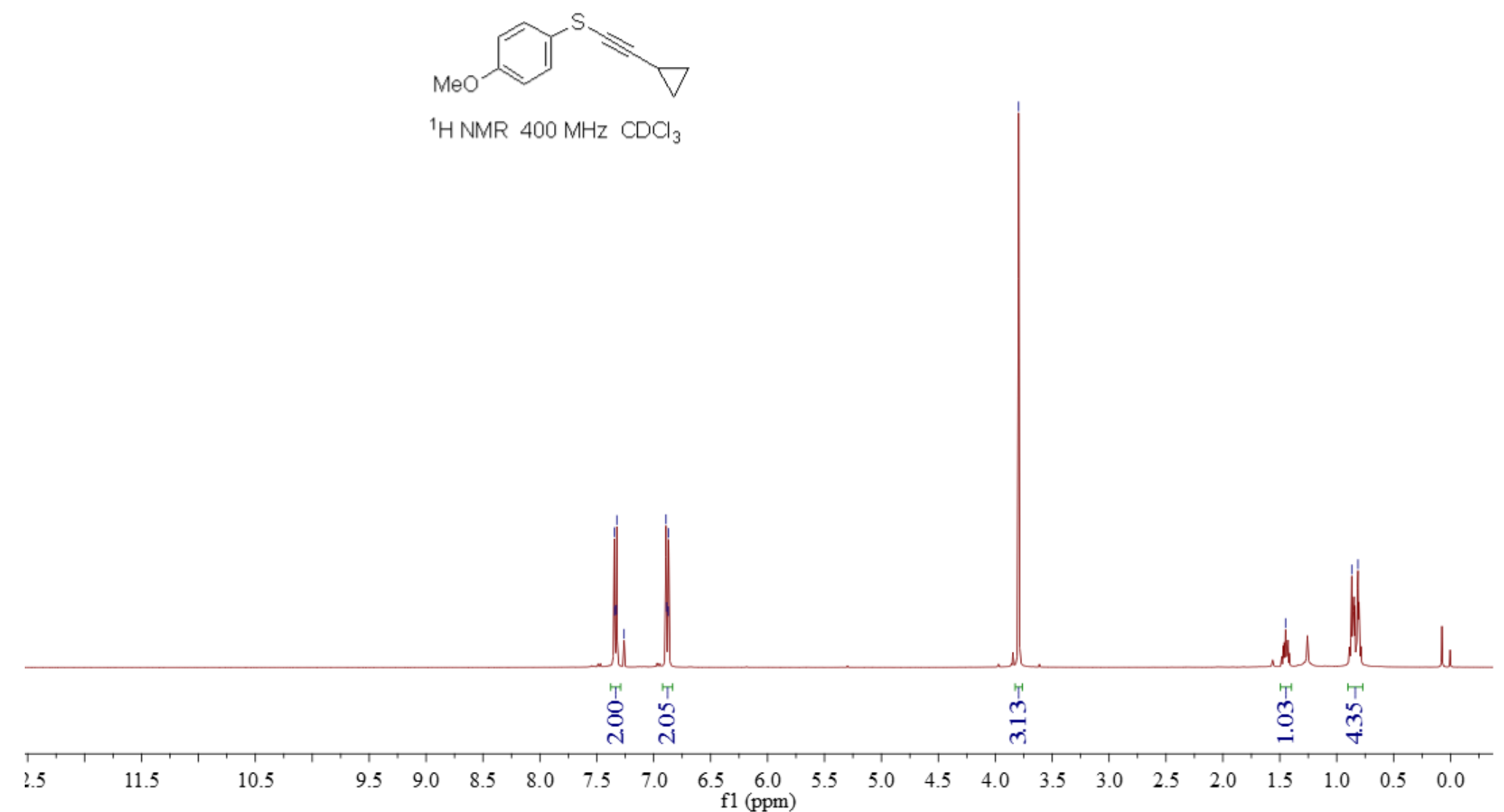



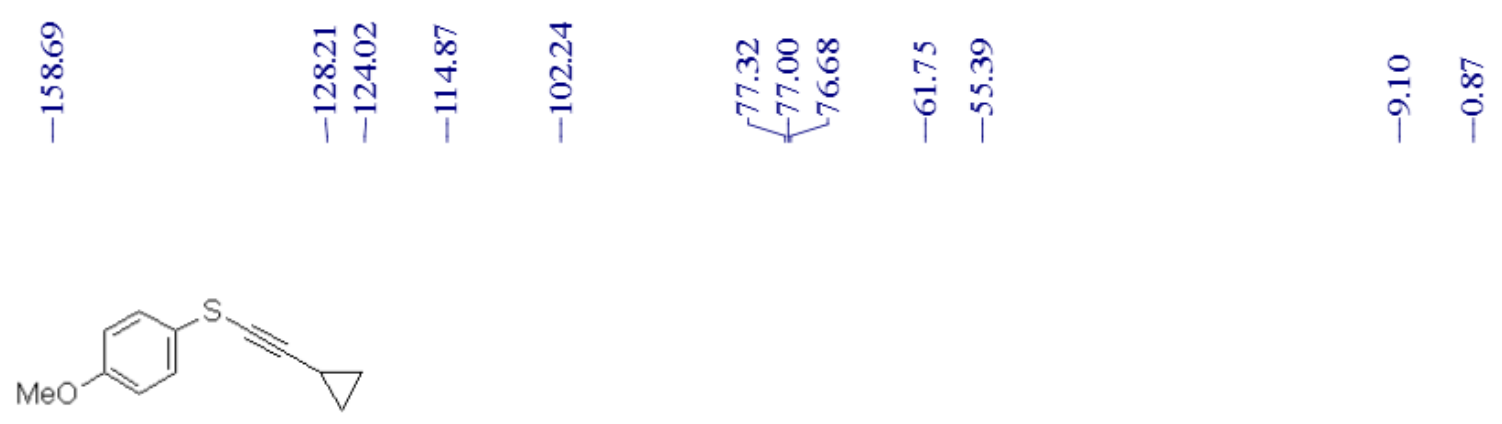

${ }^{13} \mathrm{CNMR} 100 \mathrm{MHz} \mathrm{CDCl}_{3}$
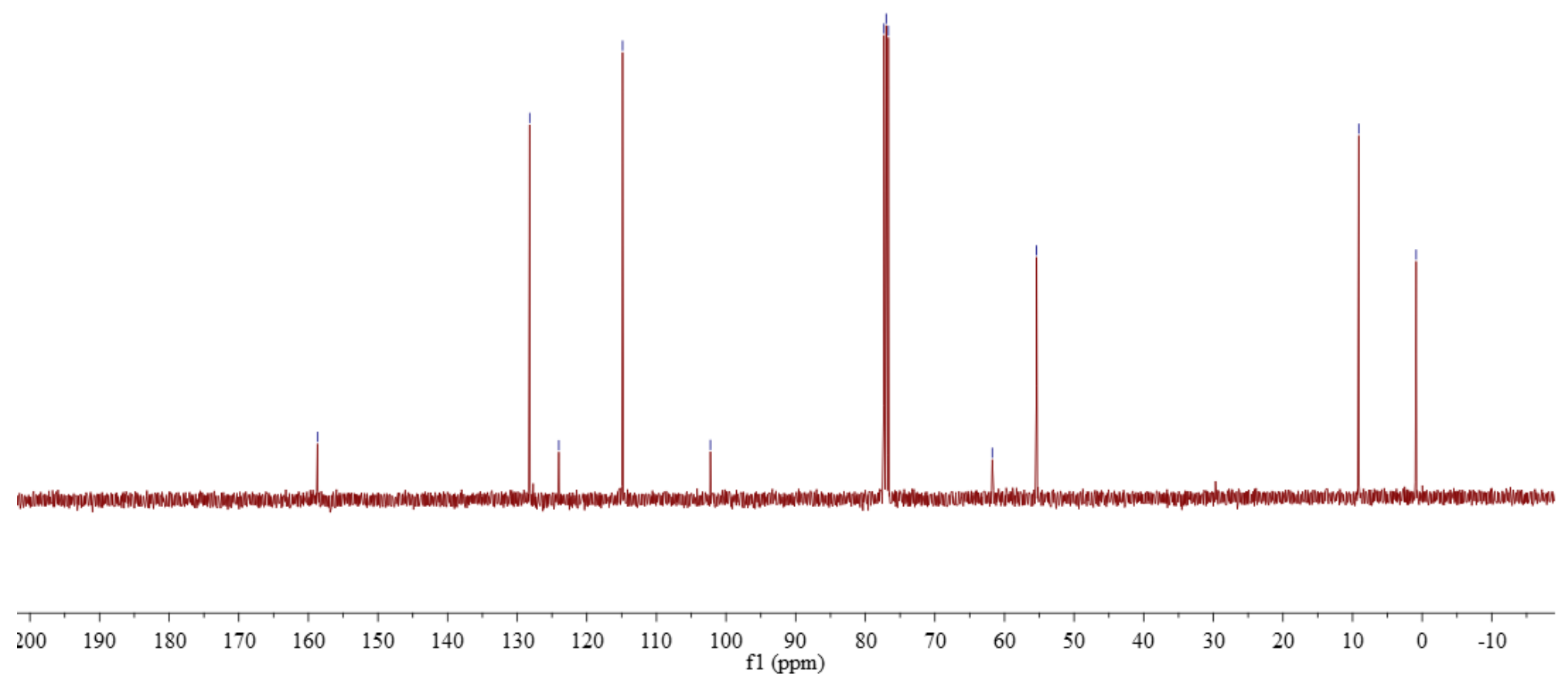
mे

${ }^{1} \mathrm{HNMR} 400 \mathrm{MHz} \mathrm{CDCl}_{3}$

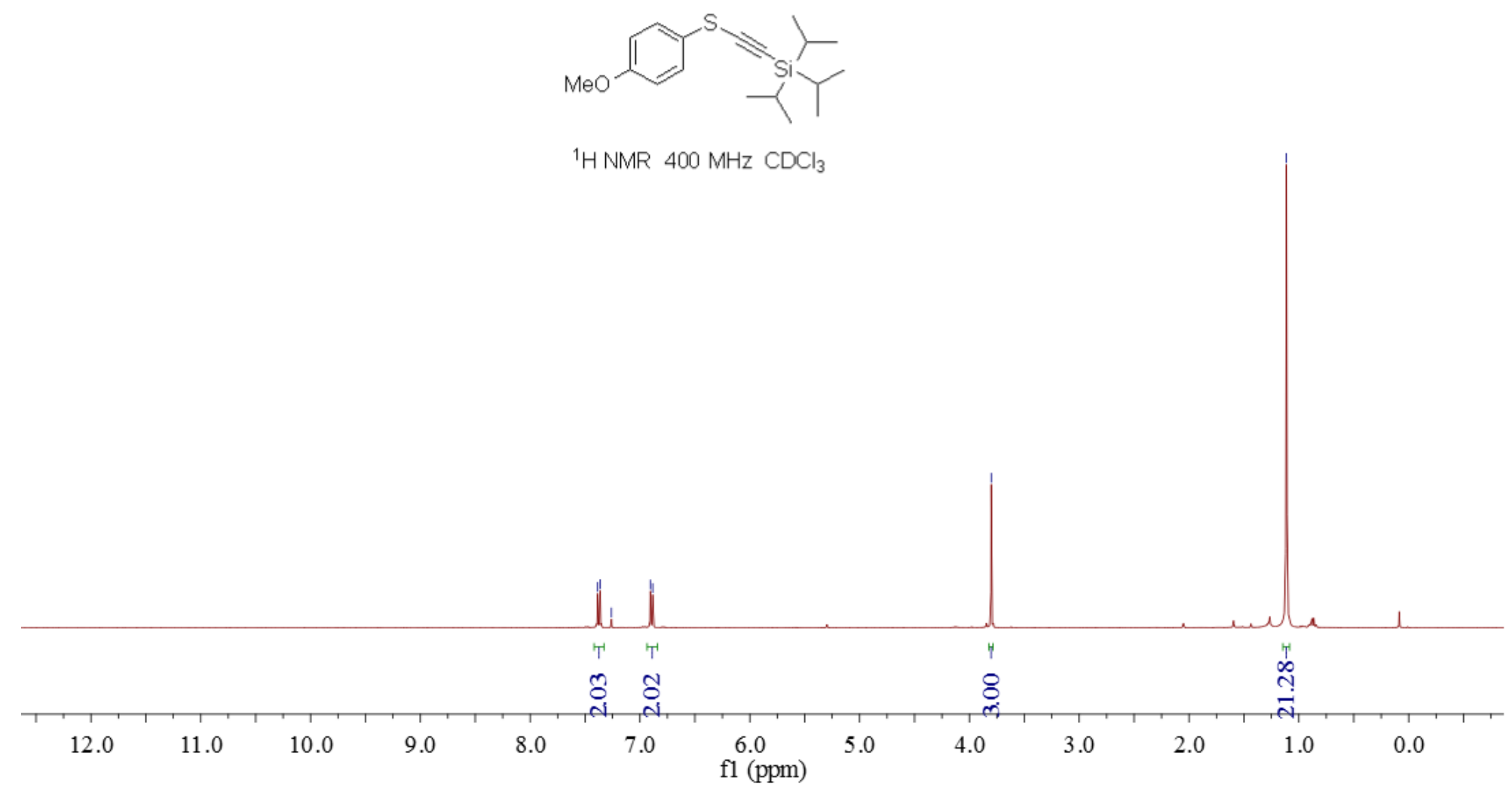

$\stackrel{\infty}{i} \quad=$ 

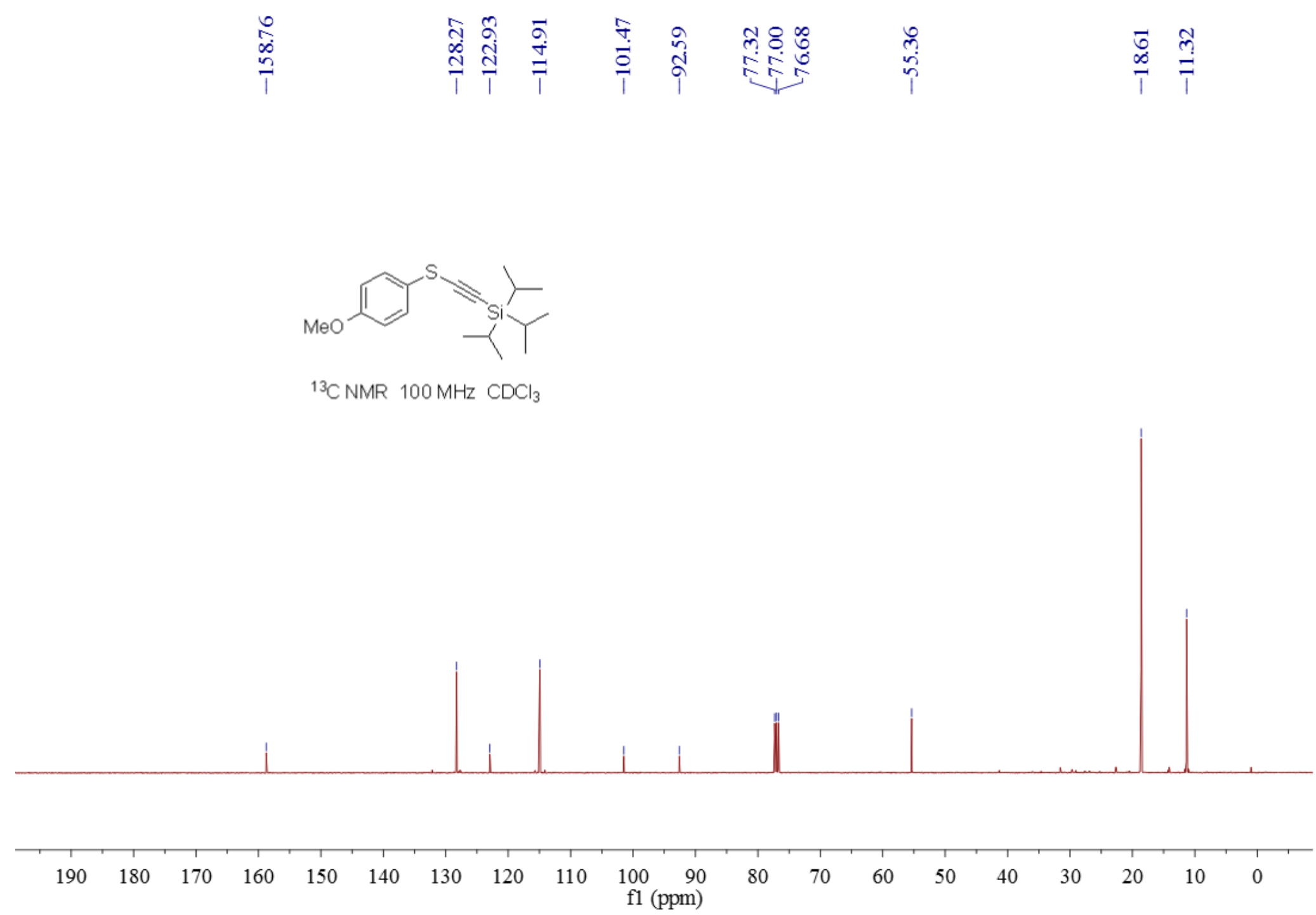


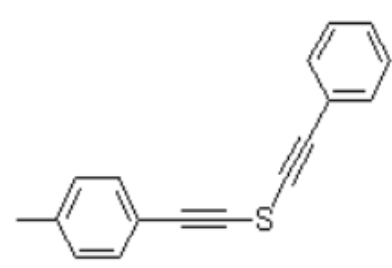

${ }^{1} \mathrm{H}$ NMR $400 \mathrm{MHz} \mathrm{CDCl}_{3}$

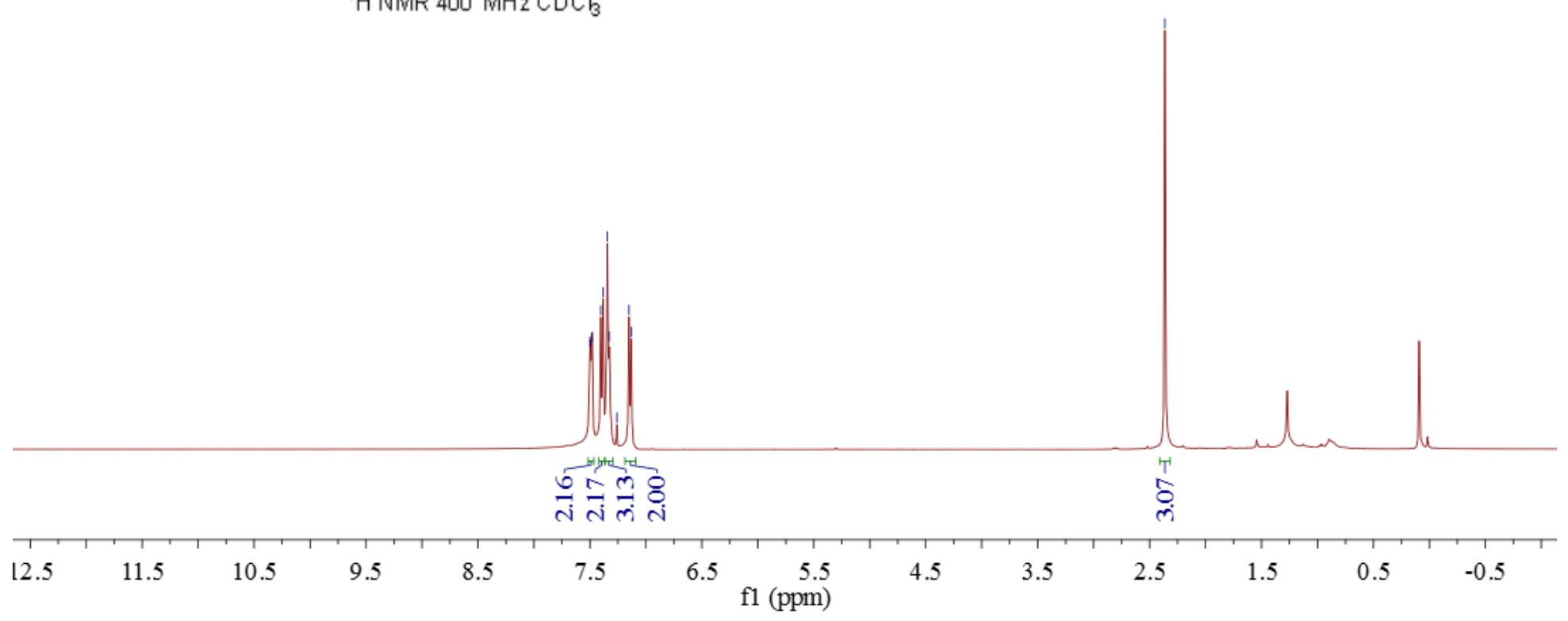




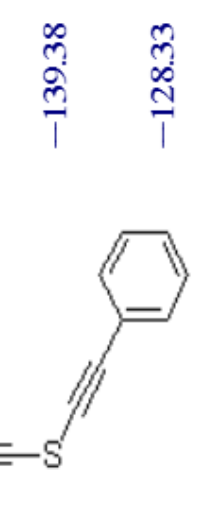

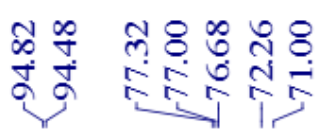

$\stackrel{n}{i}$

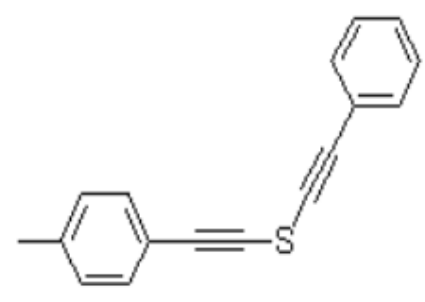

${ }^{13} \mathrm{C} \mathrm{NMR} 100 \mathrm{MHz} \mathrm{CDCl}$

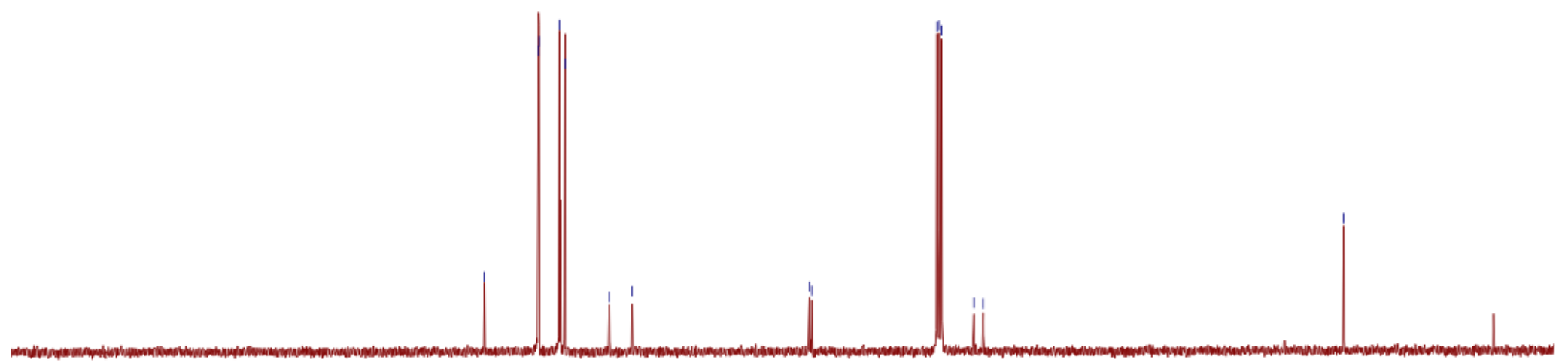

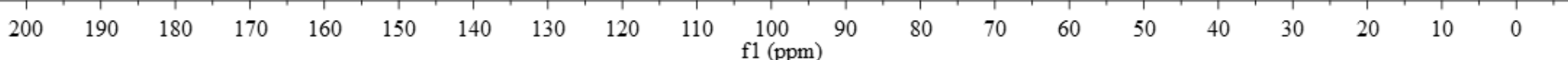




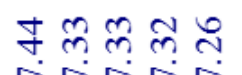

20

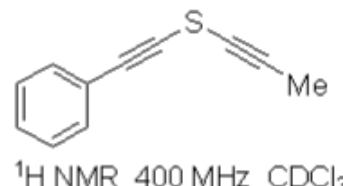

${ }^{1} \mathrm{HNMR} 400 \mathrm{MHz} \mathrm{CDCl}_{3}$

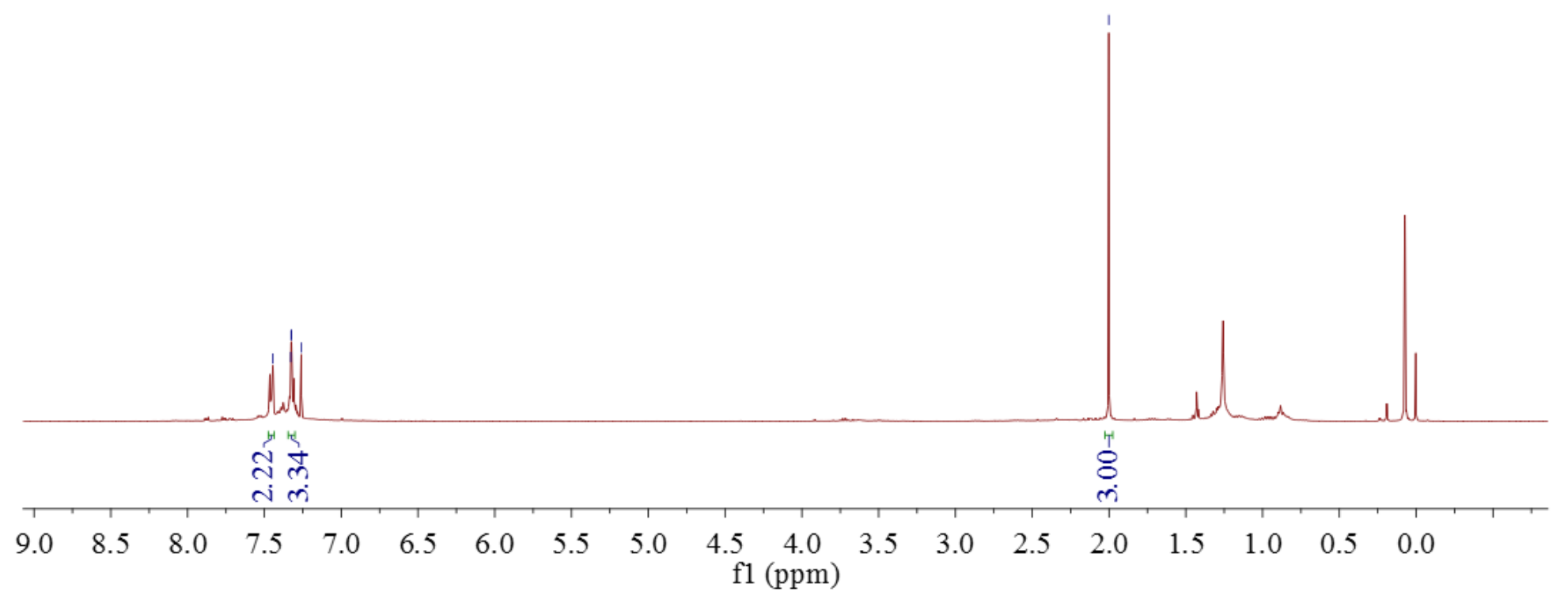




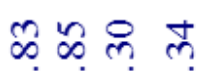

m $\stackrel{\infty}{\infty} \stackrel{\infty}{N}$

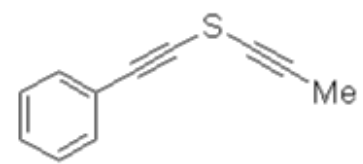

${ }^{13} \mathrm{CNMR} 100 \mathrm{MHz} \mathrm{CDCl}_{3}$

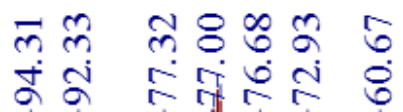

$\stackrel{\infty}{\circ}$

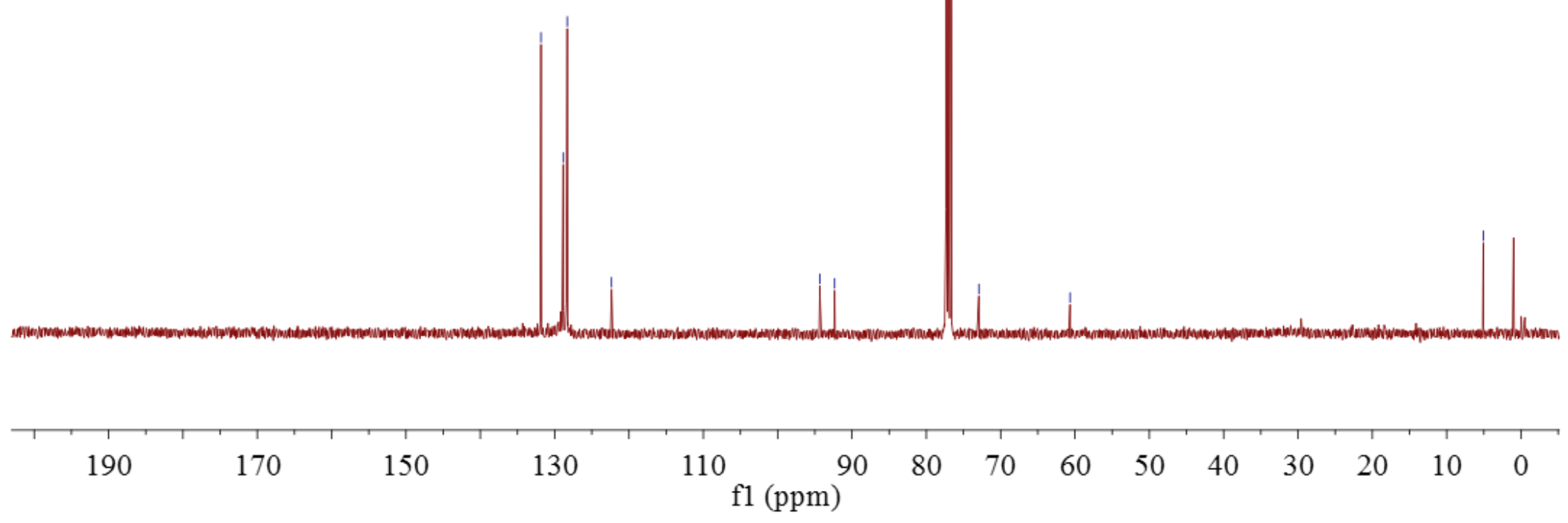




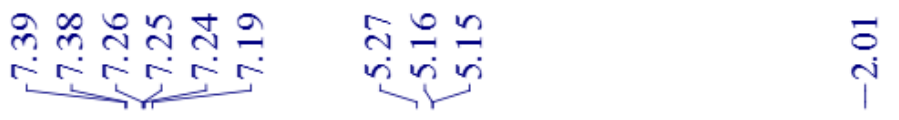

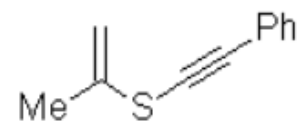

${ }^{1} \mathrm{HNMR} 400 \mathrm{MHz} \mathrm{CDCl}_{3}$

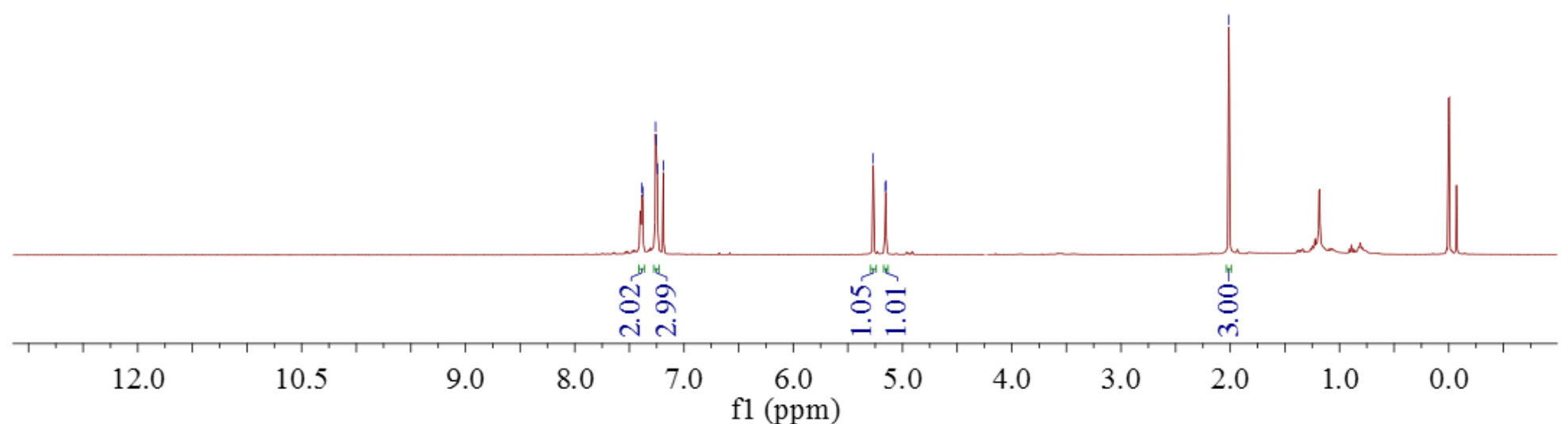



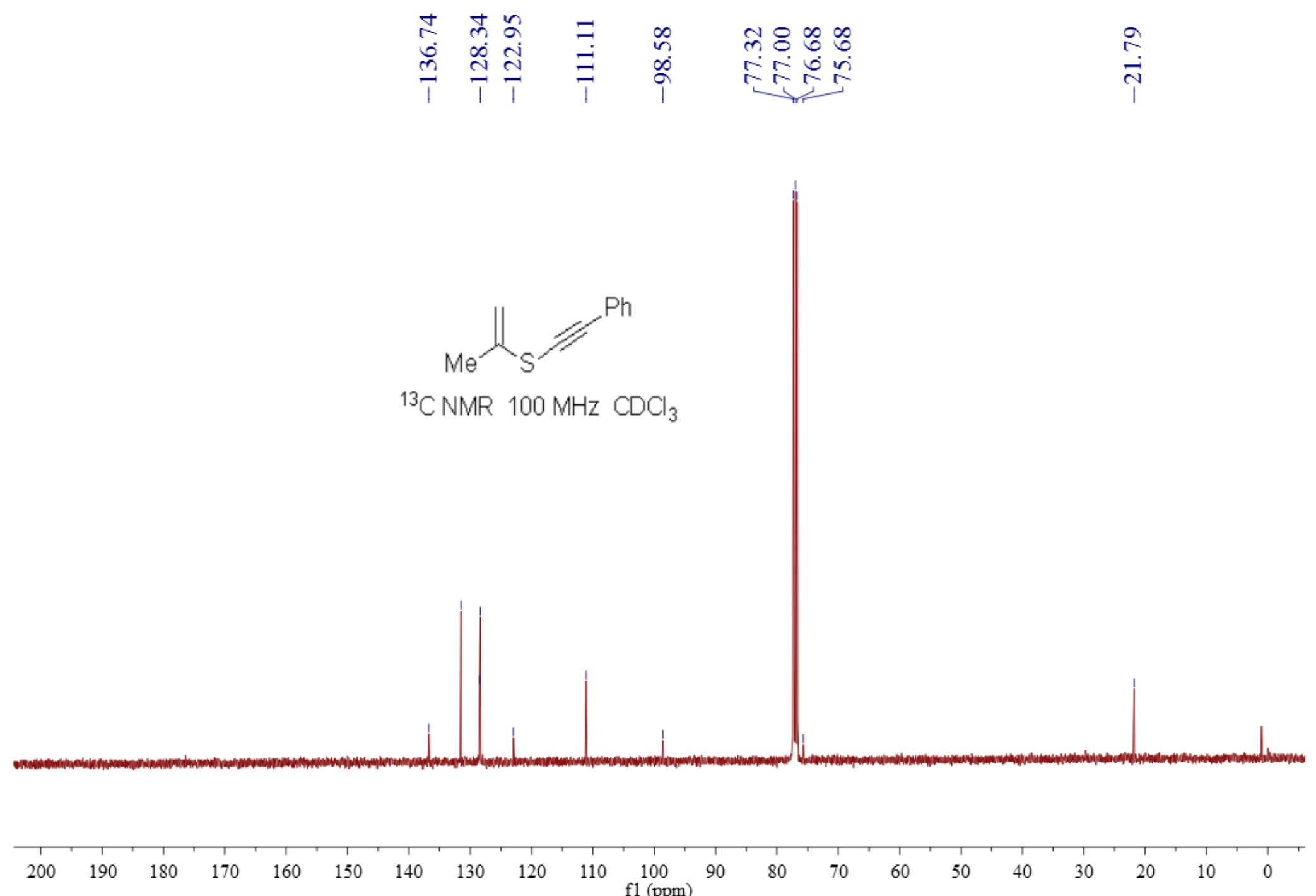


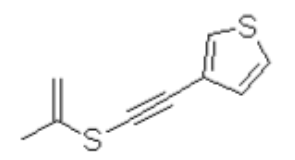

${ }^{1} \mathrm{HNMR} \quad 400 \mathrm{MHz} \quad \mathrm{CDCl}_{3}$

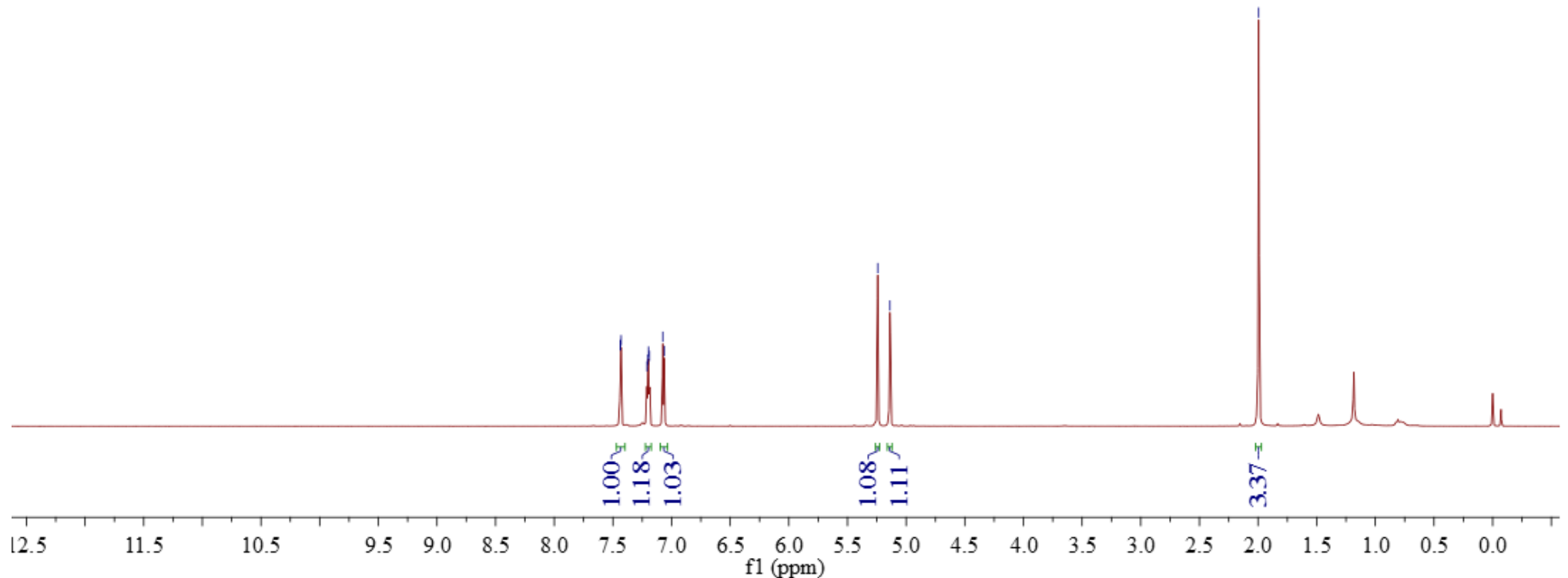




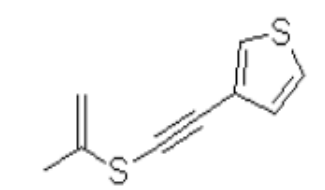

${ }^{13} \mathrm{CNMR} \quad 100 \mathrm{MHz} \quad \mathrm{CDCl}_{3}$

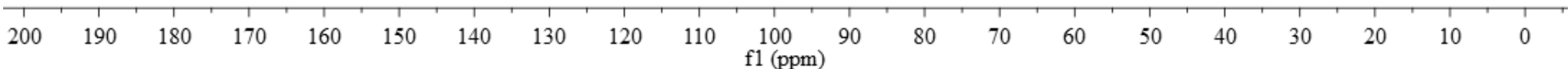



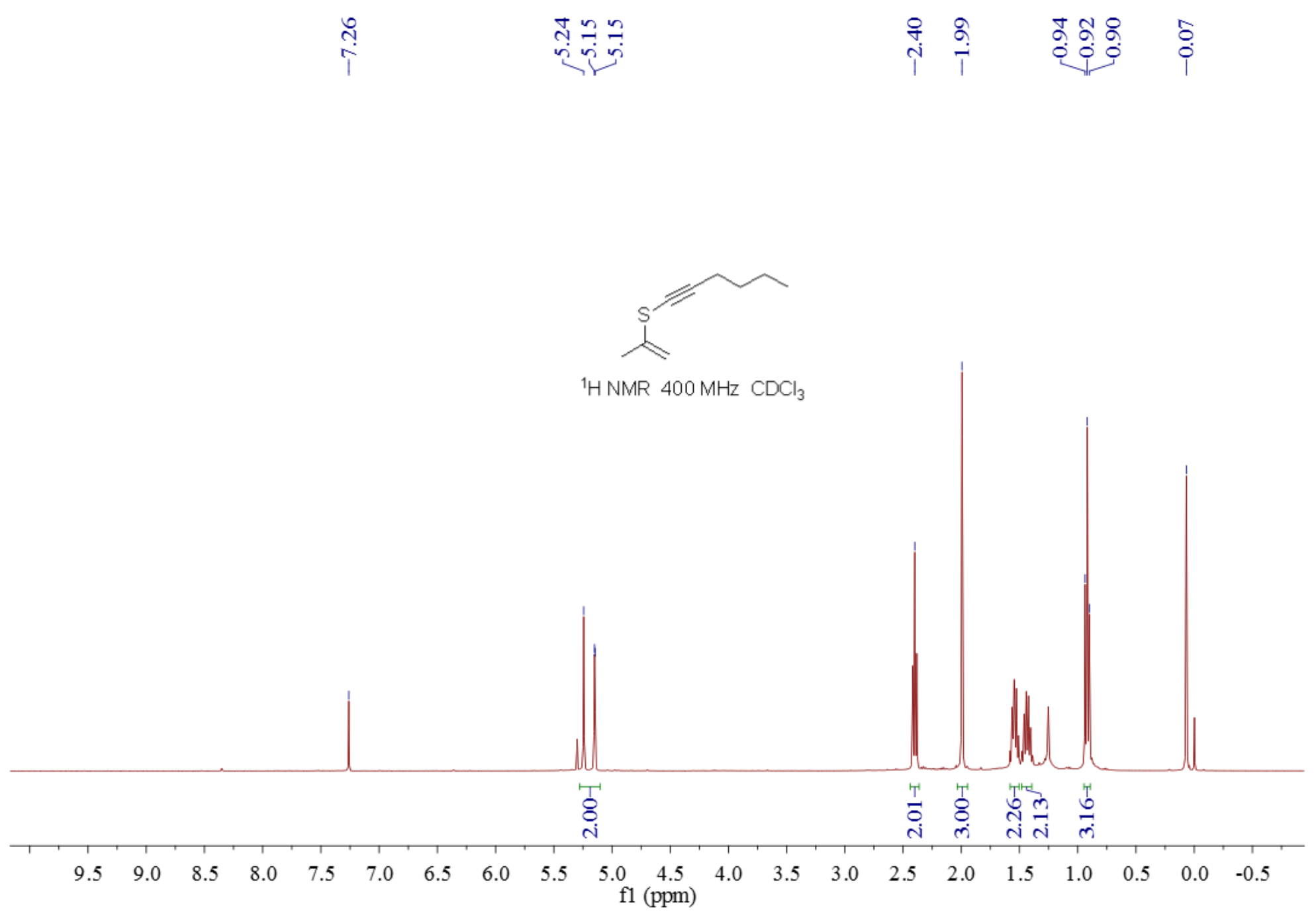


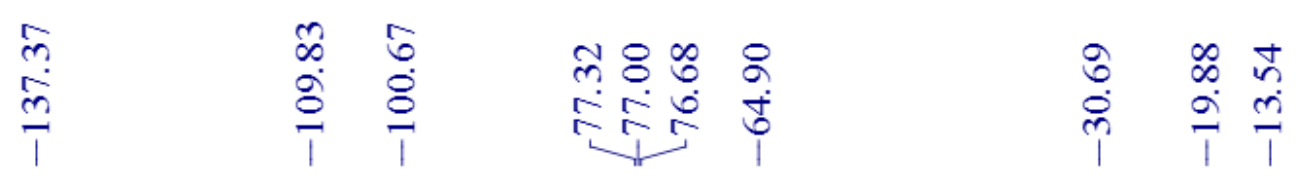

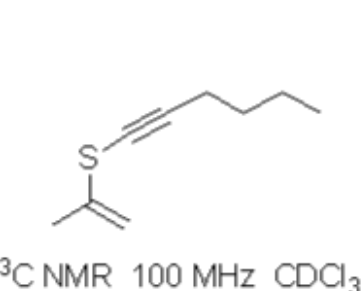

${ }^{13} \mathrm{CNMR} 100 \mathrm{MHz} \mathrm{CDCl}_{3}$
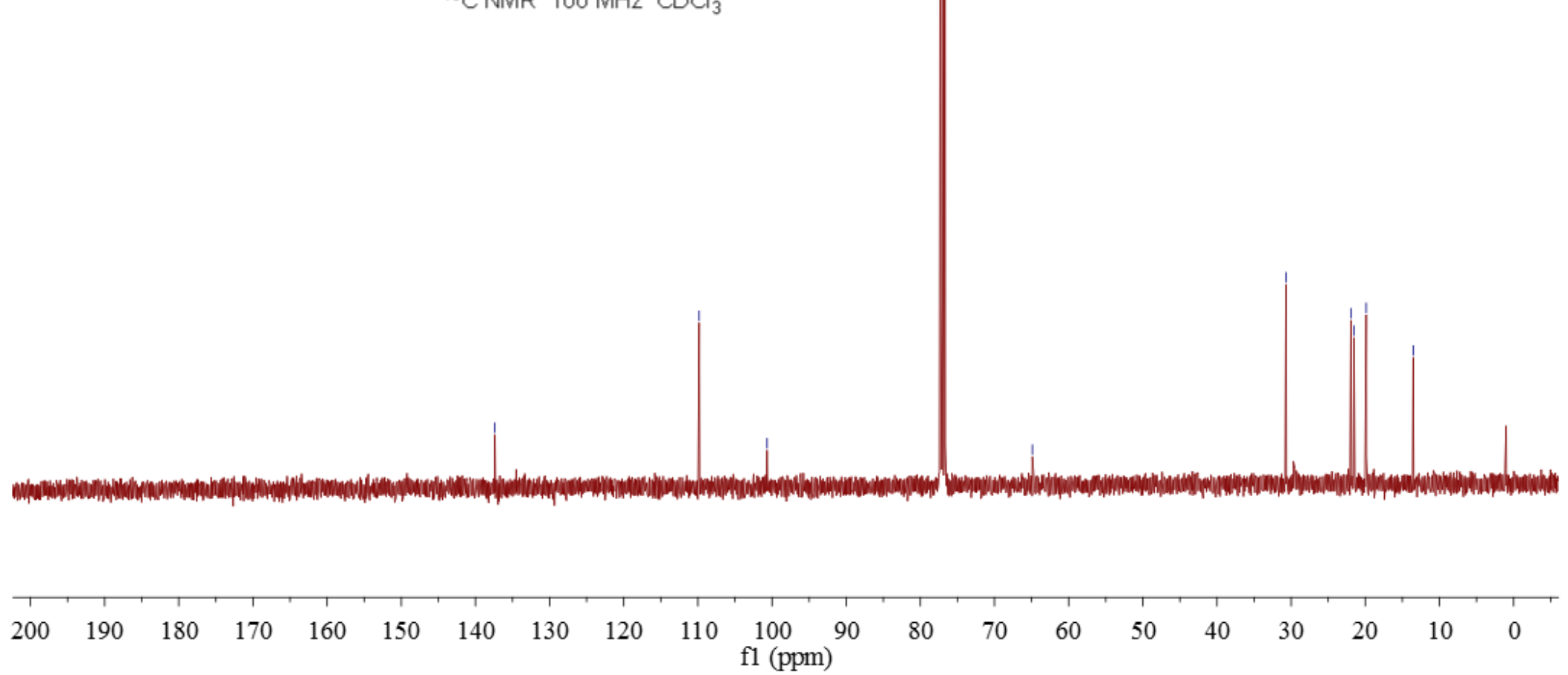

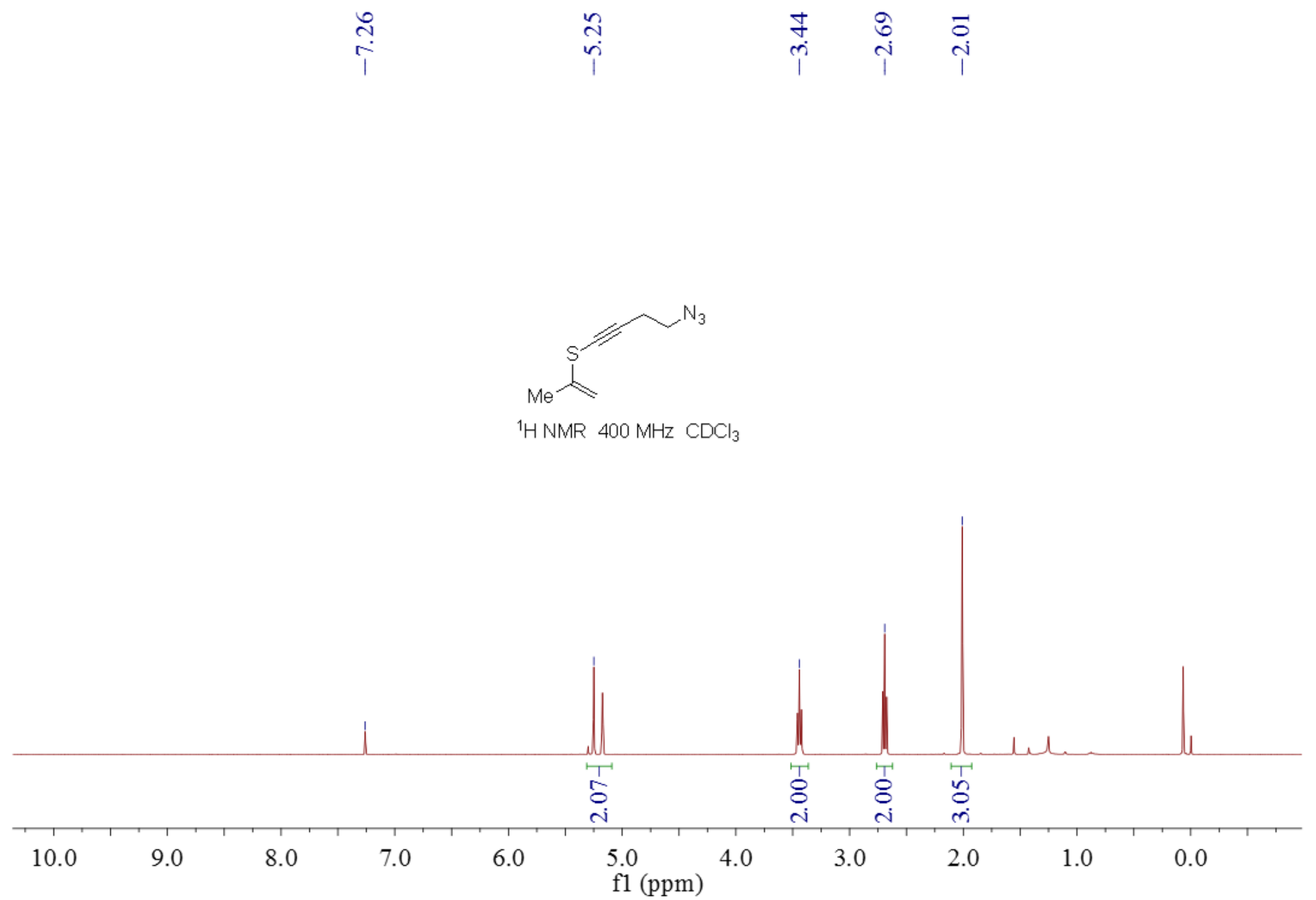


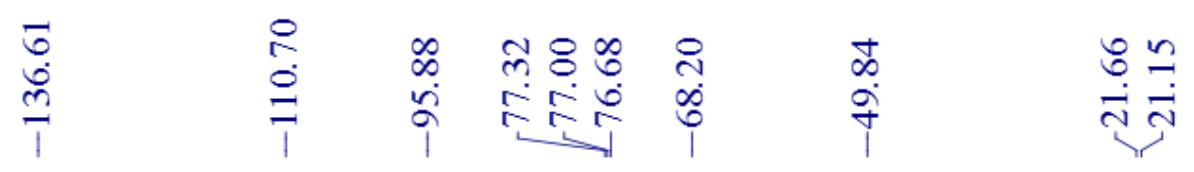

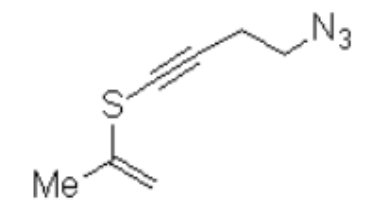

${ }^{13} \mathrm{CNMR} 100 \mathrm{MHz} \mathrm{CDCl}_{3}$ 

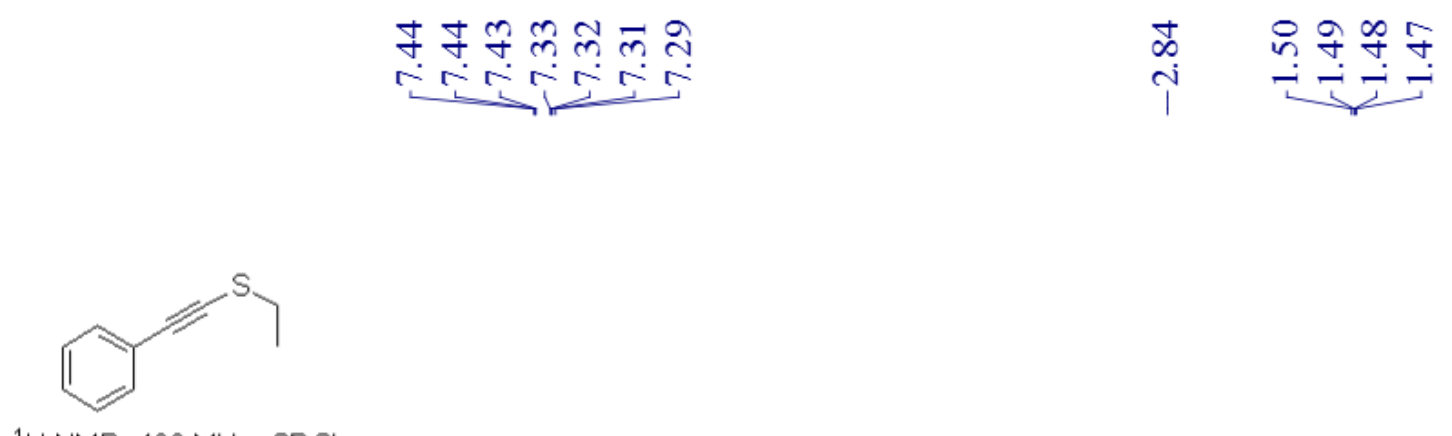

${ }^{1} \mathrm{HNMR} 400 \mathrm{MHz} \mathrm{CDCl}_{3}$

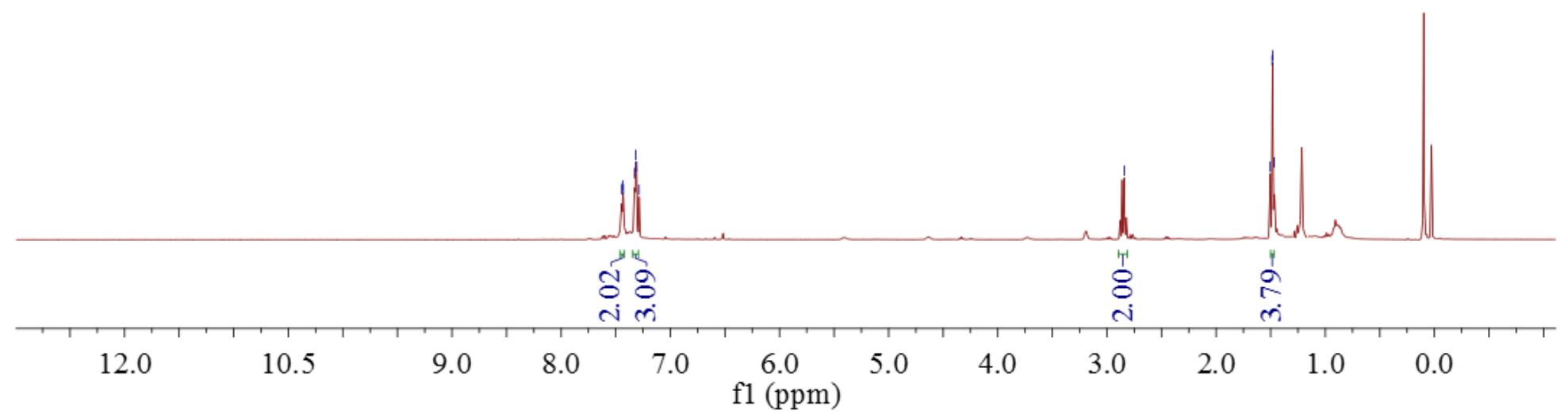


ㅇำ

穴空空

के 9200

के

$\stackrel{\frac{1}{2}}{\stackrel{+}{\pi}}$

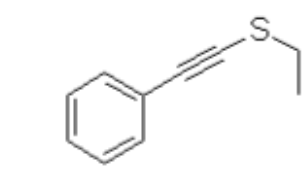

${ }^{13} \mathrm{CNMR} 100 \mathrm{MHz} \mathrm{CDCl}_{3}$

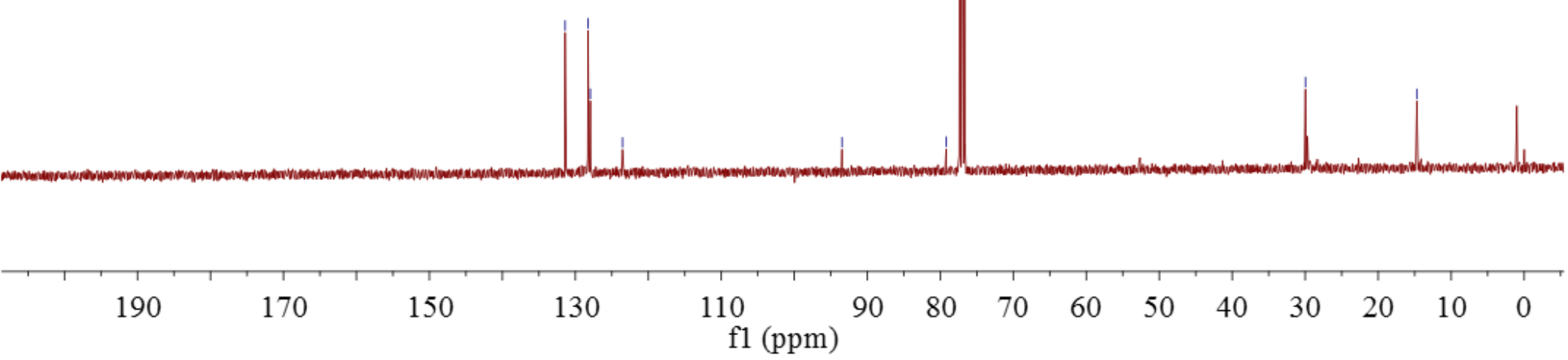



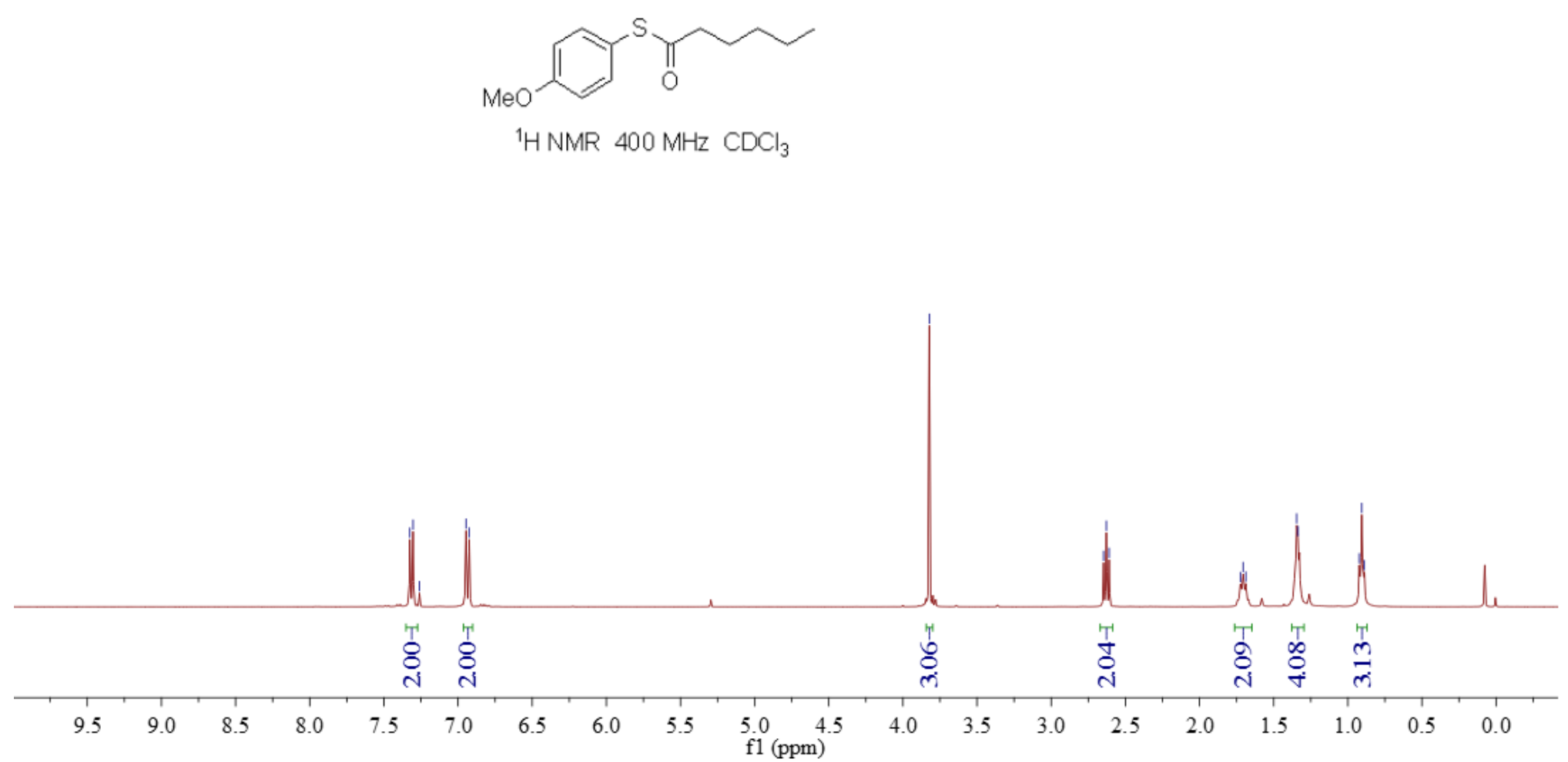


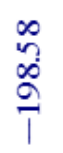

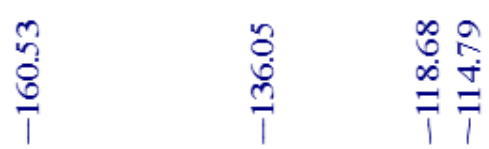

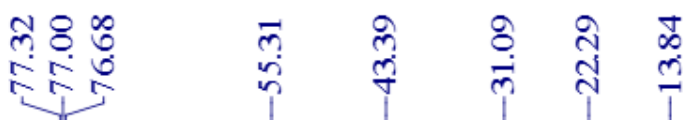
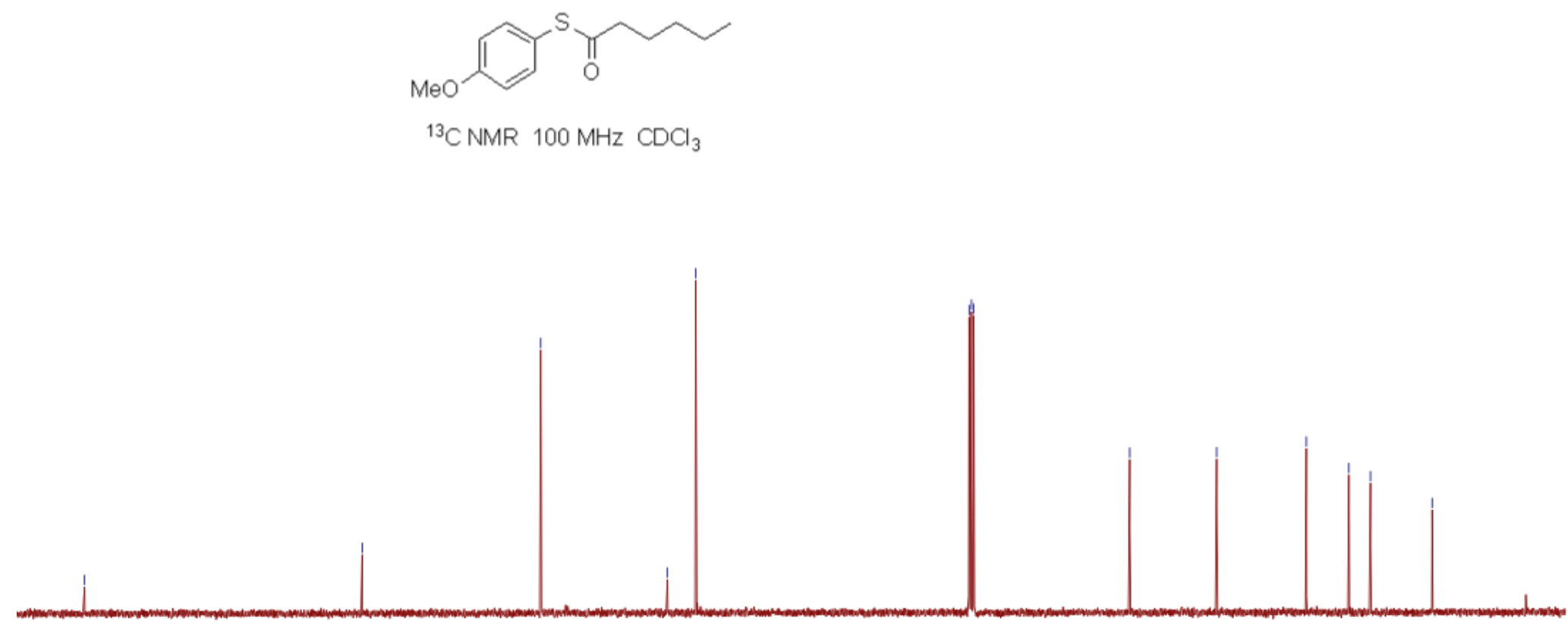

$\begin{array}{lllllllllll}200 & 190 & 180 & 170 & 160 & 150 & 140 & 130 & 120 & 110 & 100\end{array}$ f1 (ppm) 


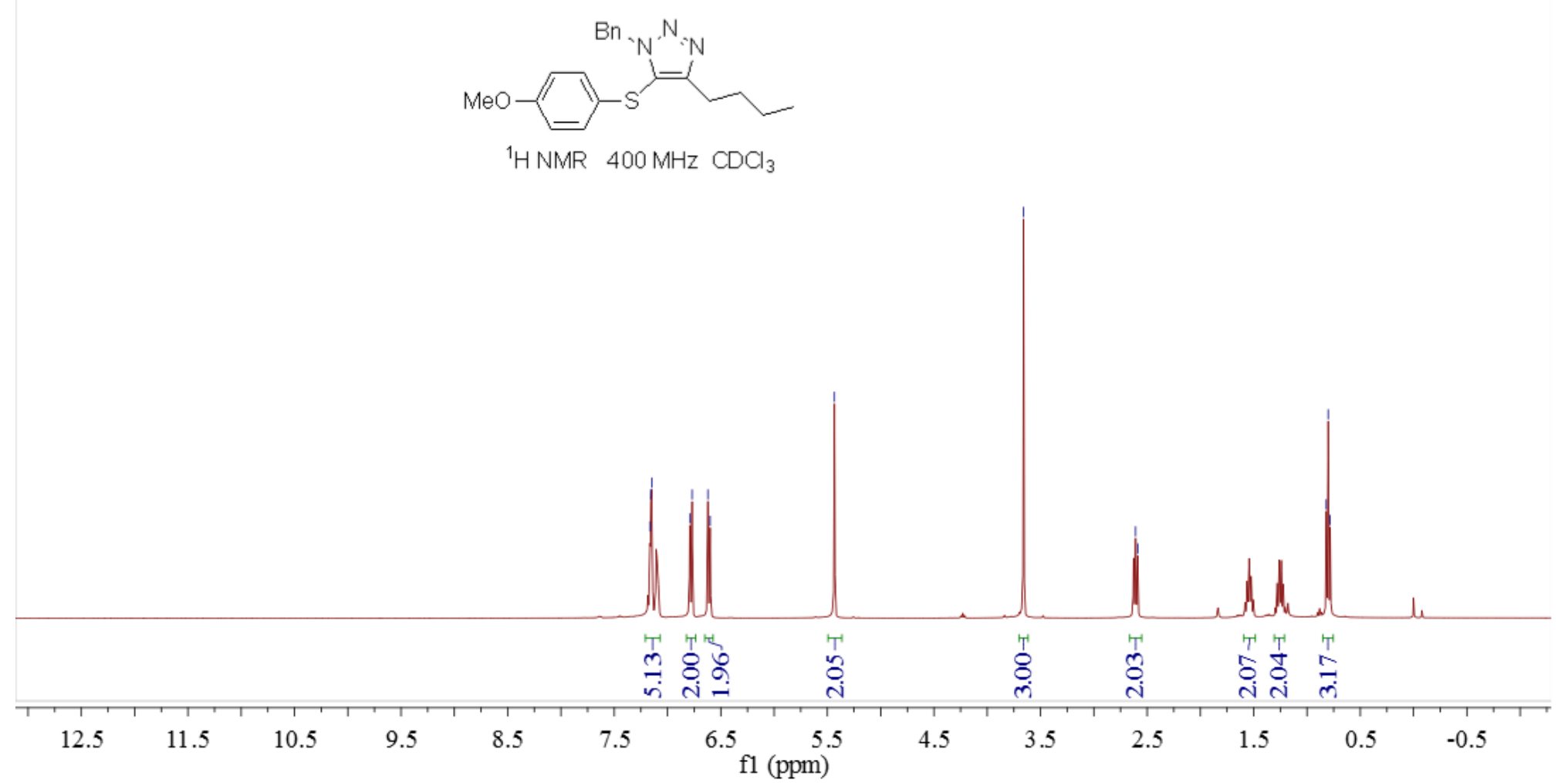




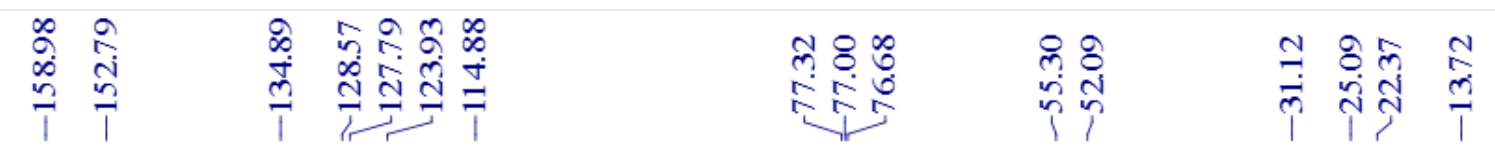
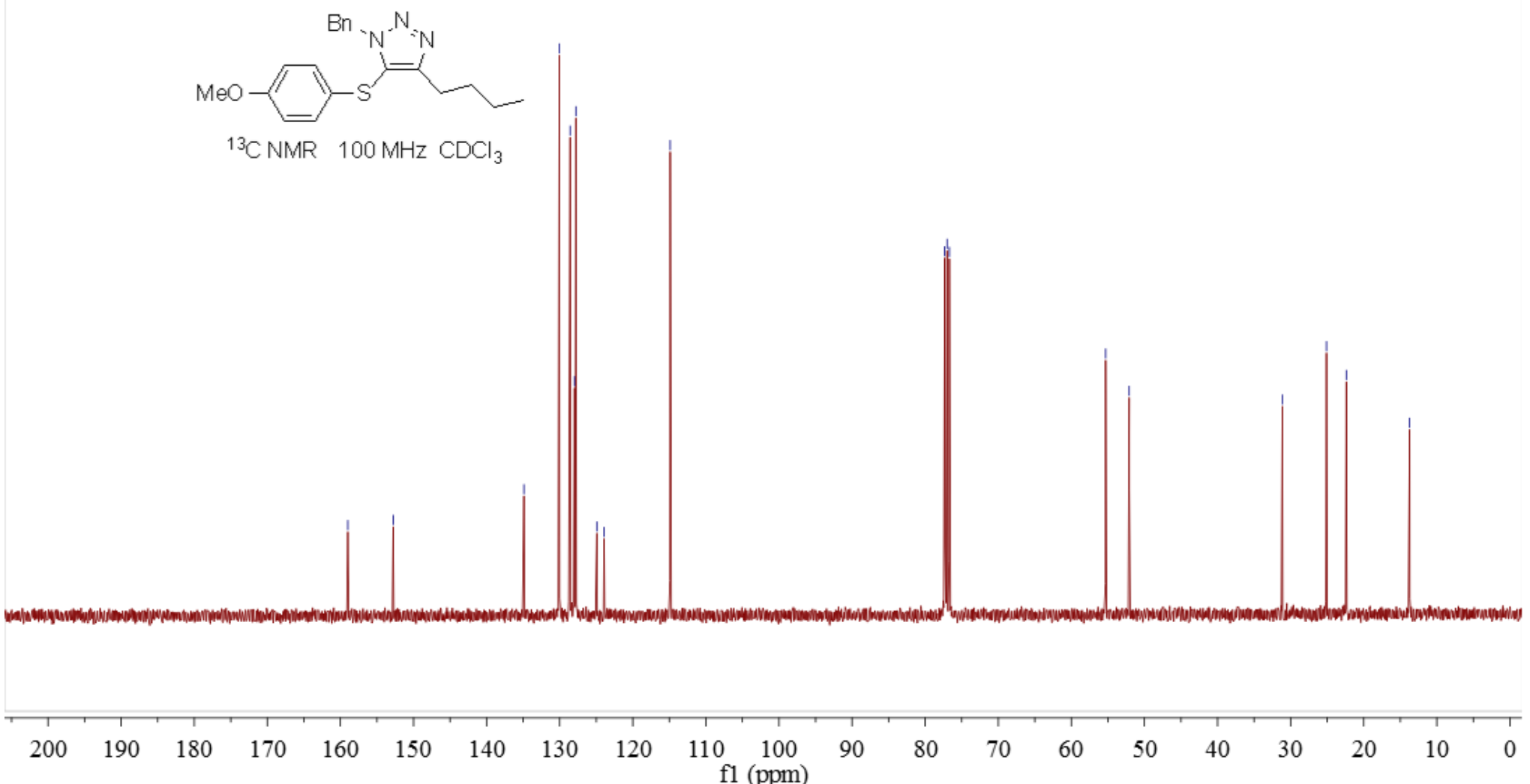